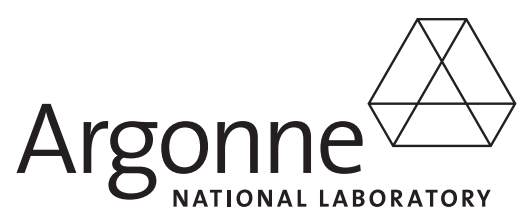

ANL/EVS/AGEM/TR-12-02

\title{
Annual Report of Monitoring at Barnes, Kansas, in 2011
}

Environmental Science Division

USDA

United States Department of Agriculture 
About Argonne National Laboratory

Argonne is a U.S. Department of Energy laboratory managed by UChicago Argonne, LLC under contract DE-AC02-06CH11357. The Laboratory's main facility is outside Chicago, at 9700 South Cass Avenue, Argonne, Illinois 60439. For information about Argonne and its pioneering science and technology programs, see www.anl.gov.

\section{Availability of This Report}

This report is available, at no cost, at http://www.osti.gov/bridge. It is also available on paper to the U.S. Department of Energy and its contractors, for a processing fee, from:

U.S. Department of Energy

Office of Scientific and Technical Information

P.O. Box 62

Oak Ridge, TN 37831-0062

phone (865) 576-8401

fax (865) 576-5728

reports@adonis.osti.gov

\section{Disclaimer}

This report was prepared as an account of work sponsored by an agency of the United States Government. Reference herein to any specific commercial product, process, or service by trade name, trademark, manufacturer, or otherwise, does not necessarily constitute or imply its endorsement, recommendation, or favoring by the United States Government or any agency thereof. The views and opinions of document authors expressed herein do not necessarily state or reflect those of the United States Government or any agency thereof, Argonne National Laboratory, or UChicago Argonne, LLC. 


\section{Annual Report of Monitoring at Barnes, Kansas, in 2011}

by

Applied Geosciences and Environmental Management Section

Environmental Science Division, Argonne National Laboratory

February 2012

USDA 


\section{Contents}

Notation.

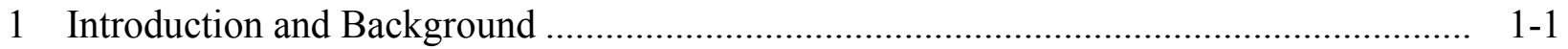

2 Conceptual Site Model ................................................................................ $2-1$

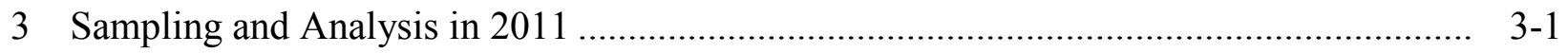

3.1 Measurement of Groundwater Levels........................................................... 3-1

3.2 Well Sampling and Analyses ...................................................................

3.3 Handling and Disposal of Investigation-Derived Waste ..................................... 3-2

3.4 Quality Control for Sample Collection, Handling, and Analysis .......................... 3-2

4 Results and Discussion .................................................................................. $4-1$

4.1 Groundwater Level Data .................................................................................... 4-1

4.2 Analytical Results for Volatile Organic Compounds in Groundwater Samples and Lateral Distribution of the Contaminants.................................................. 4-2

5 Conclusions, Observations, and Recommendations ................................................ $5-1$

5.1 Conclusions.................................................................................... $5-1$

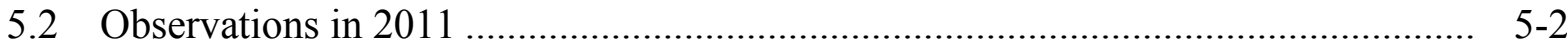

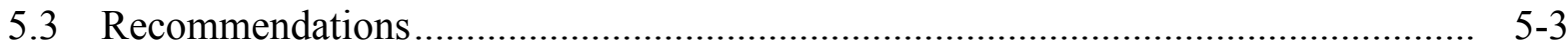

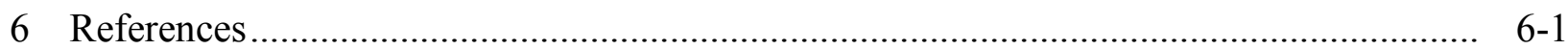

Appendix A: Sampling Activities and Field Measurements in 2011 ............................. A-1

Appendix B: Results from the AGEM Laboratory for Dual Analyses of Samples Collected in 2011 and for Quality Control Samples

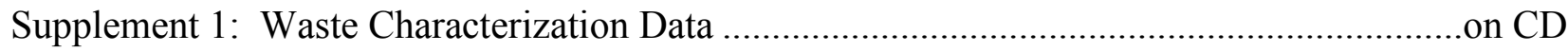

Supplement 2: Sample Documentation from TestAmerica Laboratories, Inc........................ CD 


\section{Figures}

1.1 Location of Washington County and Barnes, Kansas ........................................ 1-7

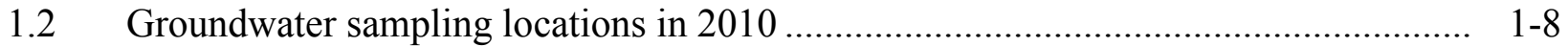

3.1 Wells equipped with data loggers for automatic water level monitoring in November 2011

3.2 Groundwater sampling locations in September 2011

4.1 Potentiometric surface map depicting the groundwater flow direction in the deep aquifer zone under static (non-pumping) conditions on November 18, 2011

4.2a Potentiometric surface map depicting the groundwater flow direction in the deep aquifer zone under static (non-pumping) conditions on February 2, 2010

4.2b Potentiometric surface map depicting the groundwater flow direction in the deep aquifer zone under pumping conditions on February 2, 2010

4.3a Hydrographs summarizing results of long-term water level monitoring in the deep-zone wells, January-June 2011

4.3b Hydrographs summarizing results of long-term water level monitoring in the deep-zone wells, July-November 2011

4.4 Hydrographs summarizing results of water level monitoring in the deep-zone wells, June 2011

4.5 Analytical results for carbon tetrachloride in groundwater samples collected in 2009-2011

4.6 Analytical results for chloroform in groundwater samples collected in 2009-2011.....

\section{Tables}

1.1 Summary of environmental investigation reports for Barnes .............................. $1-6$

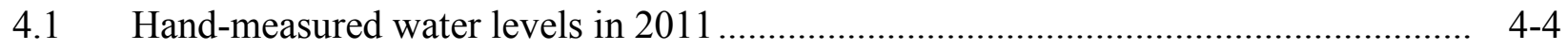

4.2 Elevation ranges measured for the three aquifer zones, 2007-2011 _...................... 4-5 


\section{Tables (Cont.)}

4.3 Analytical results from the AGEM Laboratory for volatile organic compounds in groundwater samples, 2006-2011

A.1 Sequence of groundwater sampling activities in 2011

A.2 Field measurements for groundwater samples, 2006-2011

B.1 Analytical results from the AGEM Laboratory for quality control samples collected to monitor sample collection and handling activities in 2011

B.2 Results for verification organic analyses during groundwater monitoring in $2011 \ldots \ldots$. 


\section{Notation}

AGEM Applied Geosciences and Environmental Management

AMSL above mean sea level

BGL below ground level

BTOC below top of casing

${ }^{\circ} \mathrm{C} \quad$ degree(s) Celsius

CCC Commodity Credit Corporation

CD compact disc

COC chain of custody

EPA U.S. Environmental Protection Agency

$\mathrm{ft} \quad$ foot (feet)

gal gallon(s)

hr hour(s)

in. inch(es)

KDHE Kansas Department of Health and Environment

L liter(s)

$\mu \mathrm{g} / \mathrm{kg} \quad$ microgram(s) per kilogram

$\mu \mathrm{g} / \mathrm{L} \quad$ microgram(s) per liter

$\mu \mathrm{S} / \mathrm{cm} \quad$ microsiemen(s) per centimeter

$\mathrm{mg} / \mathrm{L} \quad$ milligram(s) per liter

min minute

$\mathrm{mV} \quad$ millivolt(s)

ND not detected

PWS public water supply

RBSL risk-based screening level

USDA U.S. Department of Agriculture

VOC volatile organic compound 


\section{Annual Report of Monitoring at Barnes, Kansas, in 2011}

\section{Introduction and Background}

Barnes, Kansas, is a small rural community (population approximately 150) located in Washington County, in north-central Kansas (Figure 1.1). Barnes is located in Section 9, Township 4 South, Range 5 East, at approximate latitude $39^{\circ} 43^{\prime} 0^{\prime \prime}$ north and longitude 96 $52^{\prime} 25^{\prime \prime}$ west (USGS 1968). The city lies in a transition zone between the Flint Hills and the glaciated region. The area's topography consists of gently sloping hills of Pleistocene loess $(<20 \mathrm{ft})$ overlying a shale unit and interbedded shale, limestone, and siltstone of the Permian Chase Group. Groundwater for the public water supply is obtained from wells PWS2 and PWS3 at reported depths of $155 \mathrm{ft}$ and $160 \mathrm{ft}$, respectively, located in the northwestern portion of the city. The water is produced from the bedrock aquifer of the Chase Group. Section 2 summarizes of the hydrogeologic conceptual site model.

In 1986, low levels of carbon tetrachloride were detected in public supply wells PWS2 $(2.1 \mu \mathrm{g} / \mathrm{L})$ and PWS3 $(0.5 \mu \mathrm{g} / \mathrm{L})$, below the maximum contaminant level of $5.0 \mu \mathrm{g} / \mathrm{L}$ for carbon tetrachloride in drinking water. Multiple samplings of the wells in 1986-1996 found repeated sporadic detections of carbon tetrachloride at low concentrations.

In 1996-1999, the Kansas Department of Health and Environment (KDHE) conducted two investigations to identify potential sources for the carbon tetrachloride in groundwater and to determine the extent of the contamination, as follows:

1. In 1996, a Phase I comprehensive investigation was conducted to identify potential sources for the contamination in wells PWS2 and PWS3. The KDHE activities focused on the two following potential source areas closest to the public wells:

- The site of the former high school, including an agricultural vocational building where chemicals including carbon tetrachloride and chloroform had been mixed and stored as part of the high school curriculum and then dumped outside (PRC 1996). Subsequently, the chemicals were inventoried and disposed of as hazardous waste through the KDHE (USD 233 1989). This site is less than $250 \mathrm{ft}$ from the public wells. 
- The site of the grain storage facility formerly operated by the Commodity Credit Corporation of the U.S. Department of Agriculture (CCC/USDA), approximately $800 \mathrm{ft}$ downgradient from the public wells. The CCC/USDA facility was in operation in 1949-1974. During this time, carbon tetrachloride-based fumigants were used for preservation of the stored grain.

As part of the 1996 comprehensive investigation, soil gas and soil samples were collected at the sites of the former high school and the former CCC/USDA grain storage facility. Groundwater samples from the public wells and local private wells were also collected. Low levels of carbon tetrachloride and chloroform were detected in the soil gas samples at both the former high school and the former CCC/USDA grain storage facility. Carbon tetrachloride was not detected in the soil samples or the water samples.

2. In 1998-1999, the KDHE conducted a Phase II comprehensive investigation to determine the extent of the groundwater contamination and the local groundwater gradient. Five monitoring wells (MW1S, MW1D, MW2D, MW3D, and MW4D) were installed to delineate the contamination previously detected in wells PWS2 and PWS3. Carbon tetrachloride was detected in well MW4D, on the former CCC/USDA grain storage property.

In 2006, the CCC/USDA assumed responsibility for the site investigation of the carbon tetrachloride contamination, which could in part potentially be linked to historical use of carbon tetrachloride-based fumigants at its former facility. Initially, the CCC/USDA developed and implemented a work plan for targeted groundwater sampling and monitoring well installation (Argonne 2006). The investigation and subsequent monitoring (Argonne 2008a-d, 2009a-b, 2010, 2011) were performed by the Environmental Science Division of Argonne National Laboratory. The reports of environmental investigations at Barnes are summarized in Table 1.1. The results have been reported in detail in the cited references.

The CCC/USDA activities at Barnes have been as follows:

1. In 2006-2007, the CCC/USDA conducted a comprehensive targeted investigation at and near its former property. A network of 28 monitoring 
wells was established at 19 locations (including the 5 wells previously installed by the KDHE; Argonne 2008a). The investigation results indicated that carbon tetrachloride contamination is present in groundwater at low to moderate levels in the vicinity of the former CCC/USDA grain storage facility and extends westward at diminishing concentrations toward the public wells.

2. In November 2007, the CCC/USDA began periodic sampling to monitor the identified carbon tetrachloride contamination in groundwater. The sampling was initially conducted quarterly to verify that the contaminant migration pattern does not pose an imminent risk to the public wells. The sampling is now conducted annually.

3. In 2009, a contingency interim measure (Argonne 2009c) was approved by the KDHE (2009).

4. Through 2010, sampling was conducted in a network of 28 individual monitoring wells (at 19 distinct locations), 2 public water supply wells, and 1 private well (Figure 1.2). On the basis of an evaluation of the data collected in 2006-2009 (Argonne 2010), including a trend analysis of the site contamination and its migration, the KDHE (2010b) concurred that future monitoring will occur annually, with twice-yearly sampling of the two public water supply wells in service. The KDHE (2010a) also agreed to decrease the number of wells to be sampled.

The key results and findings of prior investigations at Barnes indicate the following:

- No soil contamination at concentrations above the method quantitation limit of $10 \mu \mathrm{g} / \mathrm{kg}$ has been detected on the former CCC/USDA property. Trace concentrations of carbon tetrachloride $(<10 \mu \mathrm{g} / \mathrm{kg})$ were detected at three locations on the former CCC/USDA property. These trace concentrations will not result in higher concentrations in groundwater, and therefore the soil on the former CCC/USDA property is not an ongoing significant source for carbon tetrachloride contamination. 
- The levels of carbon tetrachloride contamination detected in groundwater are relatively low and limited in extent, and the boundaries of the carbon tetrachloride plume have been defined. The results of the 2006-2007 targeted investigation and the subsequent monitoring events (Argonne 2008a-d, 2009a,b, 2010, 2011) demonstrated the presence of carbon tetrachloride contamination in groundwater at levels exceeding the RBSL of $5.0 \mu \mathrm{g} / \mathrm{L}$ for this compound. The contaminant plume extends from the former CCC/USDA property northwestward, toward the Barnes public water supply wells. Longterm monitoring of the groundwater levels and the contaminant distribution has confirmed that pumping of the public wells affects the direction of groundwater flow. When these wells are not pumping, the direction of groundwater flow is to the northeast. However, when the wells are pumping, groundwater flow is directed to the northwest, toward the public wells.

- The distribution of carbon tetrachloride and chloroform in both soil and groundwater suggests that natural degradation has occurred. The highest level of carbon tetrachloride in soil was found at location MW5, which lies between the former CCC/USDA facility and a surface drainage ditch extending toward the northeast (in the direction of natural groundwater flow when the public wells are not pumping). Carbon tetrachloride was distributed throughout the MW5 soil profile at 28-71 ft BGL (below ground level), from a maximum concentration of $40 \mu \mathrm{g} / \mathrm{kg}$ at $39 \mathrm{ft}$ BGL to an estimated concentration (below the method quantitation limit) of $2.3 \mu \mathrm{g} / \mathrm{kg}$ at $71.5 \mathrm{ft}$ BGL. Concentrations of chloroform (the primary degradation product of carbon tetrachloride) showed an increasing trend with depth. Only low-level residual concentrations of carbon tetrachloride remained in soils on the former CCC/USDA property. The highest levels of carbon tetrachloride in groundwater are found on the former CCC/USDA property, but the highest chloroform concentrations are found at well locations to the northeast (in the direction of groundwater flow when the public wells are not pumping). At these locations, relatively lower dissolved oxygen and oxidation-reduction potential values (conducive to reductive dechlorination) have consistently been measured. 
- Barnes residents have access to an uncontaminated public water supply. Residents obtain their water from two public water supply wells northwest of the former CCC/USDA property. The carbon tetrachloride plume is well defined downgradient, between the former CCC/USDA property and the public wells. Although trace levels of carbon tetrachloride have been detected in well PWS2, no increasing trend of contaminant migration toward the public wells has been indicated. Sentinel wells MW1D and MW17 provide data sufficient to monitor contaminant migration. A contingency interim measure work plan (Argonne 2009c) approved by the KDHE (2009) involving a granular activated carbon system could be implemented if necessary.

This present report documents the results of monitoring conducted in 2011. Sampling of the approved monitoring well network and the two public water supply wells was conducted on September 28-29 2011. The two public wells were sampled again on December 12, 2011. 
TABLE 1.1 Summary of environmental investigation reports for Barnes.

Report Reference

KDHE reports

Comprehensive investigation of the public water supply

Results of quarterly monitoring of the public water supply in March 2000

BE\&K 1999

BE\&K 2000

CCC/USDA reports

Work plan for targeted groundwater sampling

Results of the 2006-2007 investigation

Results of groundwater monitoring in November 2007

Results of groundwater monitoring in March 2008

Results of groundwater monitoring in July 2008

Results of groundwater monitoring in October 2008

Results of groundwater monitoring in March-June 2009

Contingency interim measure for the public water supply

Results of groundwater monitoring in July-December 2009

Argonne 2006

Results of groundwater monitoring in 2010

Argonne 2008a

Argonne 2008b

Argonne 2008c

Argonne 2008d

Argonne 2009a

Argonne 2009b

Argonne 2009c

Argonne 2010

Results of groundwater monitoring in 2010

Argonne 2011 


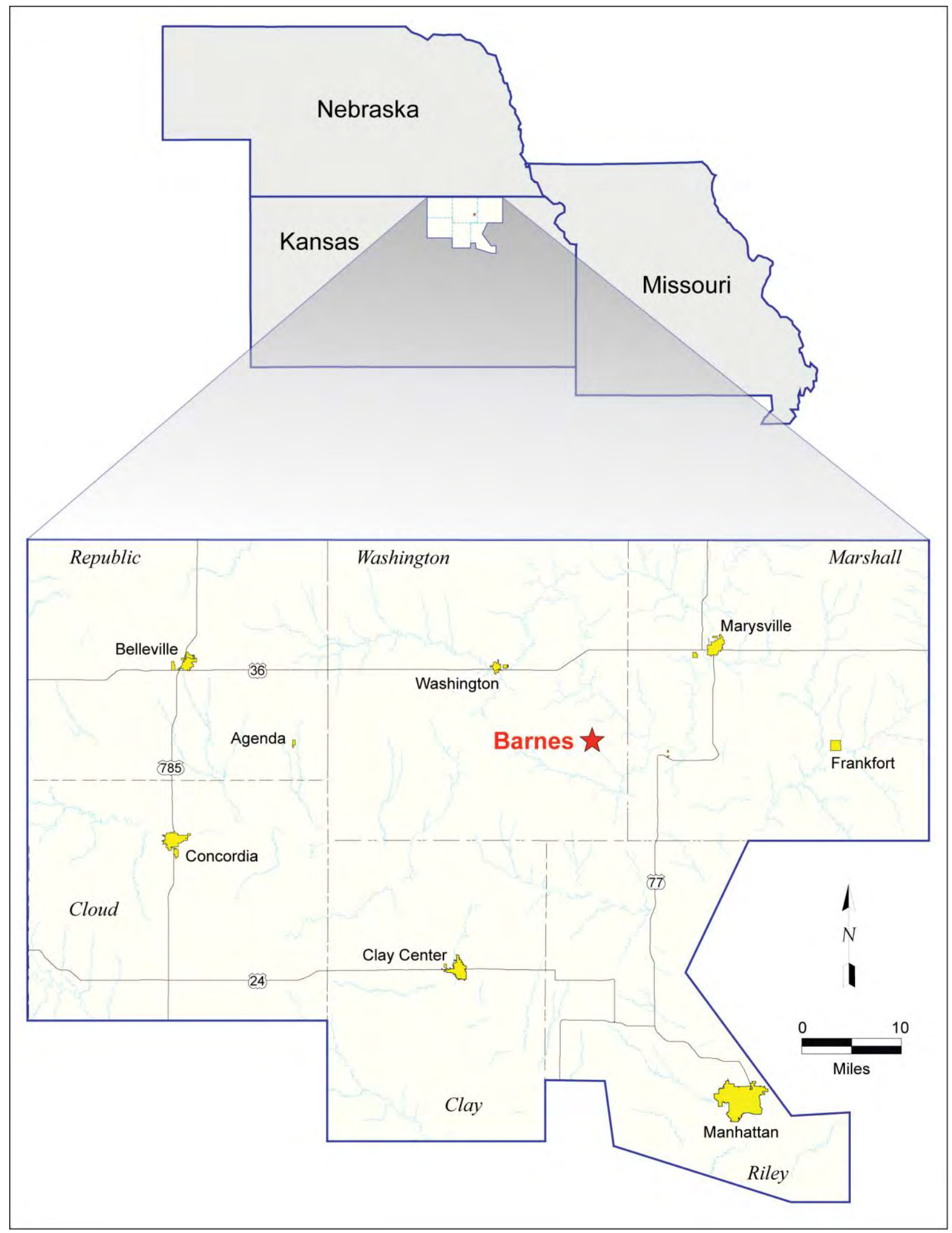

FIGURE 1.1 Location of Washington County and Barnes, Kansas. 


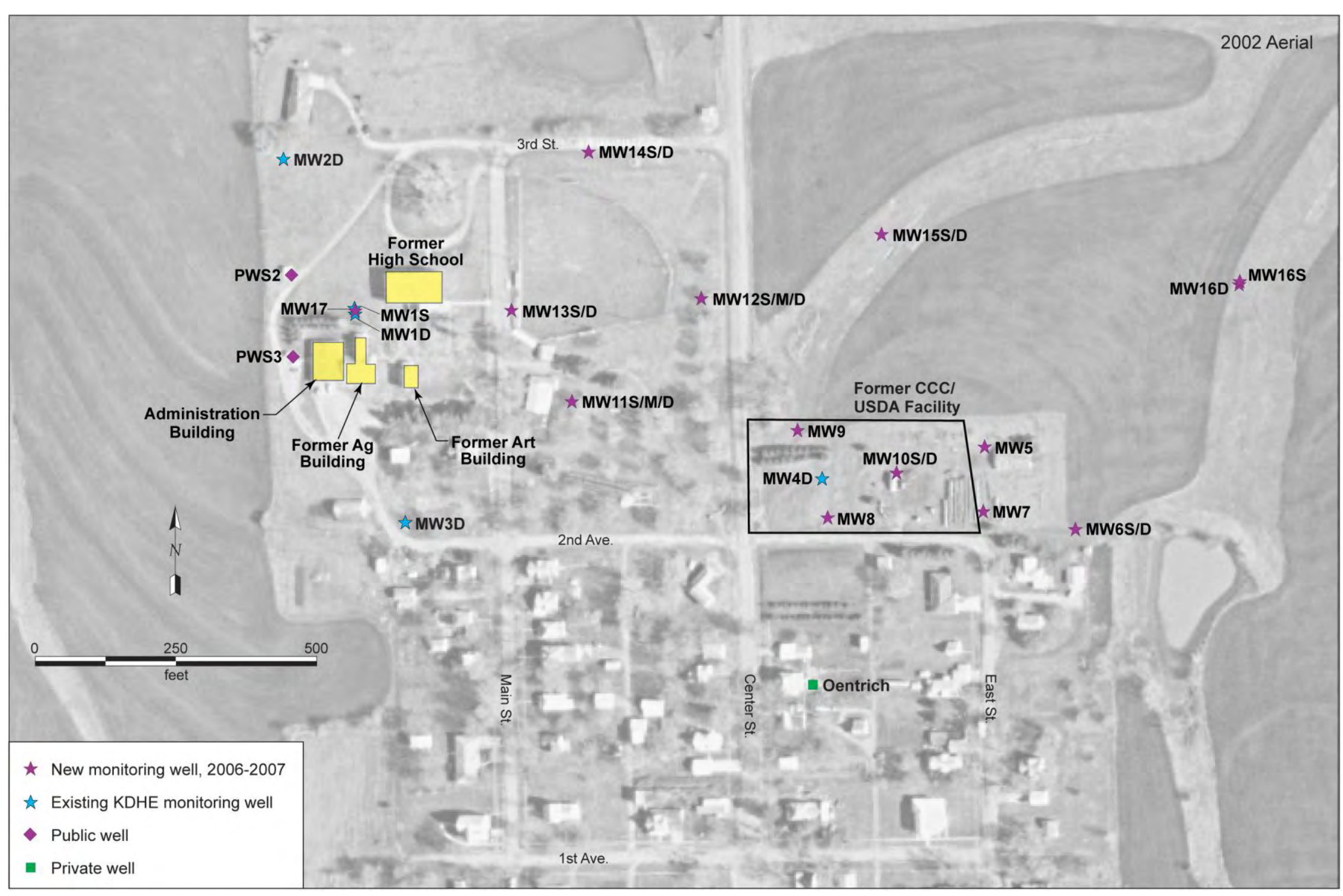

FIGURE 1.2 Groundwater sampling locations in 2010. Source of photograph: NAPP (2002). 


\section{Conceptual Site Model}

Barnes lies in a transition zone between the Flint Hills and the glaciated region. The area's topography consists of gently sloping hills of Pleistocene loess (with variations in elevation $<50 \mathrm{ft}$ ) overlying a shale unit and interbedded shale, limestone, and siltstone of the Permian Chase Group. Groundwater for the public water supply is produced from the bedrock aquifer of the Chase Group.

The site lithology and subsurface contaminant conditions were determined in the 20062007 investigation through the collection of continuous-core samples at 13 locations (MW5MW17) extending from east of the former CCC/USDA grain storage facility and westward, across the area of concern, toward the public water supply wells (Argonne 2008a). The predominant lithology consists of a thin layer of silty clay to clayey silt with fine sand in the upper 2-20 ft. This layer is underlain by highly weathered shale interbedded with thin layers of fractured limestone at depths of approximately 18-132 ft BGL. No soil contamination at concentrations above the current RBSL of $73.4 \mu \mathrm{g} / \mathrm{kg}$ for the soil-to-groundwater protection pathway was detected at any of the 13 locations. Trace concentrations $(<10 \mu \mathrm{g} / \mathrm{kg})$ of carbon tetrachloride were detected in soil at 3 locations on the former CCC/USDA property. These low concentrations would not result in higher concentrations in groundwater, and therefore the soil on the former CCC/USDA property is not considered to be a source for the carbon tetrachloride contamination in groundwater.

Groundwater is present predominantly in fractured limestone layers. Monitoring wells were installed and screened at various depths, with several locations completed as nested wells to determine contaminant concentrations at depths where water-bearing zones were indicated. Throughout the monitoring program, a detailed evaluation of the hand-measured water levels and carbon tetrachloride data has been conducted to investigate the stratigraphy of the saturated zone. The accumulated water level data confirm that three vertically distinct aquifer zones are present: shallow, intermediate, and deep. The vertical distribution of the carbon tetrachloride in groundwater indicates that the highest concentrations (approximately $50-80 \mu \mathrm{g} / \mathrm{L}$ over the course of the monitoring program to date) occur in the intermediate aquifer zone. Lower concentrations have been detected in the deep aquifer zone, and no carbon tetrachloride has been detected in the shallow zone. Trace levels of carbon tetrachloride have been detected periodically in the two public water supply wells; these wells are believed to be screened over all three aquifer zones. 
Extensive documentation of the potentiometric surface at Barnes during the targeted investigation and subsequent monitoring events (Argonne 2008a-d, 2009a,b, 2010, 2011) indicates that operation of the public water supply wells strongly influences the groundwater flow direction. The data accumulated through 2010 documented a predominant direction of groundwater flow to the northeast under non-pumping conditions. In contrast, flow was toward the northwest, in the approximate direction of the public wells, when the wells were pumping. The data demonstrated that the daily operation of the public water supply wells corresponded with drawdowns of as much as $2.25 \mathrm{ft}$ during pumping. Pumping and subsequent water level recovery periods typically ranged from $3 \mathrm{hr}$ to $7 \mathrm{hr}$ in duration, resulting in groundwater levels (and apparent flow directions) that shifted relatively continuously throughout much of each day. For this reason, water level data collected by the automatic recorders, which are coincident in time at all monitored locations, provided the primary basis for determining the topology of the potentiometric surface at any point in the cycles of groundwater pumping and recovery.

The automatic recorder data have also provided critical information needed to evaluate the hydrologic regime. The data accumulated for recorders installed in deep-zone wells indicate the presence of both vertical and lateral influences on the local hydraulic gradients. Intermediatezone wells equipped with automatic water level recorders have obtained detailed data on the potential temporal variability of the hydraulic heads in this aquifer zone. 


\section{Sampling and Analysis in 2011}

\subsection{Measurement of Groundwater Levels}

Since 2006, data recorders have been gathering long-term data on the groundwater elevation and gradient at selected monitoring wells across the investigation area. The data loggers record water levels continuously at 60 -min intervals. Water level data collected by the automatic recorders, which are coincident in time at all monitored locations, have provided the primary basis for determination of the topology of the potentiometric surface at any point in the cycles of groundwater pumping and recovery. In addition, manual water level measurements are made in conjunction with sampling and recorder downloads.

In 2011, a total of 12 wells were monitored for water levels, at the locations shown in Figure 3.1. The hand-measured and automatically recorded groundwater level data are presented and discussed in Section 4.1. After multiple recorder failures in 2010, the CCC/USDA recommended restoring a network of 12 recorders (Argonne 2011). This was completed in November 2011. The network will be maintained at this level.

\subsection{Well Sampling and Analyses}

The groundwater sampling event on September 28-29, 2011, involved 12 monitoring wells (MW1D, MW2D, MW4D, MW5, MW8, MW10S, MW10D, MW12M, MW13S, MW13D, MW14S, MW17) and the two operating public wells (PWS2 and PWS3). Sampling of the two public water supply wells was also conducted on December 12, 2011. The well locations are shown in Figure 3.2. A chronological summary of the field activities in 2011 is in Appendix A, Table A.1.

Before implementation of the low-flow sampling, a hand-held water level indicator was used to measure the depth to groundwater and the total depth of each well, to within $0.01 \mathrm{ft}$, from the top of the well casing. After measurement of water levels, the low-flow groundwater sampling technique, according to U.S. Environmental Protection Agency (EPA) guidelines (Puls and Barcelona 1996; Yeskis and Zavala 2002), was used to purge and sample the monitoring wells. The public water supply wells were sampled at their respective faucets after purging for 5 min (Table A.1 in Appendix A). The field measurements are in Appendix A, Table A.2. For 
public wells PWS2 and PWS3, samples of untreated ("raw") produced water were collected at the wellheads prior to mixing and introduction into the public distribution system.

Groundwater samples designated for analyses for volatile organic compounds (VOCs) were collected in appropriate laboratory containers, labeled, packaged, and chilled to $4^{\circ} \mathrm{C}$ by placement in ice-filled coolers. The samples were shipped via an overnight delivery service to the Applied Geosciences and Environmental Management (AGEM) Laboratory at Argonne for VOCs analyses with EPA Method 524.2 (EPA 1995). Aliquots of selected samples (chosen in the field) were also shipped to TestAmerica Laboratories, Inc., South Burlington, Vermont, for verification VOCs analyses according to EPA Contract Laboratory Program protocols.

The analytical results are presented and discussed in Section 4.2.

\subsection{Handling and Disposal of Investigation-Derived Waste}

Purge water generated as potentially contaminated investigation-derived waste was containerized on-site. The accumulated purge water was sampled on October 31, 2011 (along with wastewater from several other CCC/USDA sites in Kansas), and analyzed by Pace Analytical Services, Inc., Lenexa, Kansas, on November 4, 2011. Methods used were EPA Method 5030/8260 for VOCs, EPA Method 504.1 for ethylene dibromide, and EPA Method 353.2 for nitrate/nitrite nitrogen. Carbon tetrachloride was detected at 1.1 $\mu$ g.L. No other VOCs were detected. Nitrate/nitrite nitrogen was present at $6.1 \mathrm{mg} / \mathrm{L}$. The laboratory results are in Supplement 1, on the compact disc (CD) inside the back cover of this report. The water was delivered on December 19, 2011 (together with purge water from several other CCC/USDA investigation sites in Kansas), for disposal at the Sabetha publicly owned wastewater treatment plant.

\subsection{Quality Control for Sample Collection, Handling, and Analysis}

Quality assurance/quality control procedures followed during the 2011 monitoring events are described in detail in the Master Work Plan (Argonne 2002). The results are summarized as follows: 
- Sample collection and handling activities were monitored by the documentation of samples as they were collected and the use of chain-ofcustody forms and custody seals to ensure sample integrity during handling and shipment.

- Samples designated for VOCs analyses were received with custody seals intact and at the appropriate preservation temperature. All samples sent to the AGEM Laboratory were analyzed within the required holding times.

- Quality control samples collected to monitor sample-handling activities (a field blank, equipment rinsates, and trip blanks) and method blanks analyzed with the samples to monitor analytical methodologies were all free of carbon tetrachloride and chloroform contamination. Analytical results for quality control samples collected to monitor sample-handling activities are in Appendix B, Table B.1.

- Groundwater samples were analyzed for VOCs at the AGEM Laboratory by the purge-and-trap method on a gas chromatograph-mass spectrometer system. Calibration checks analyzed with each sample delivery group were required to be within $\pm 20 \%$ of the standard. Surrogate standard determinations performed on samples and blanks were within the specified range of $80-120 \%$ for all samples, in either the initial analysis or a successful reanalysis.

- Results from the AGEM Laboratory for dual analyses of the groundwater samples are in Appendix B, Table B.1. The results of the dual analyses compare well, with average relative percent difference values for carbon tetrachloride and chloroform of approximately $2 \%$ and $1 \%$, respectively, indicating consistency in the sampling and analytical methodologies.

- In accordance with the procedures defined in the Master Work Plan (Argonne 2002), groundwater samples were submitted to a second laboratory (TestAmerica) for verification analysis according to the protocols of the EPA's Contract Laboratory Program. Documentation is in Supplement 2 (on $\mathrm{CD}$ ). The results from the two laboratories compare favorably (Appendix B, 
Table B.2), with average relative percent difference values for carbon tetrachloride and chloroform of $10 \%$ and $1 \%$, respectively. 


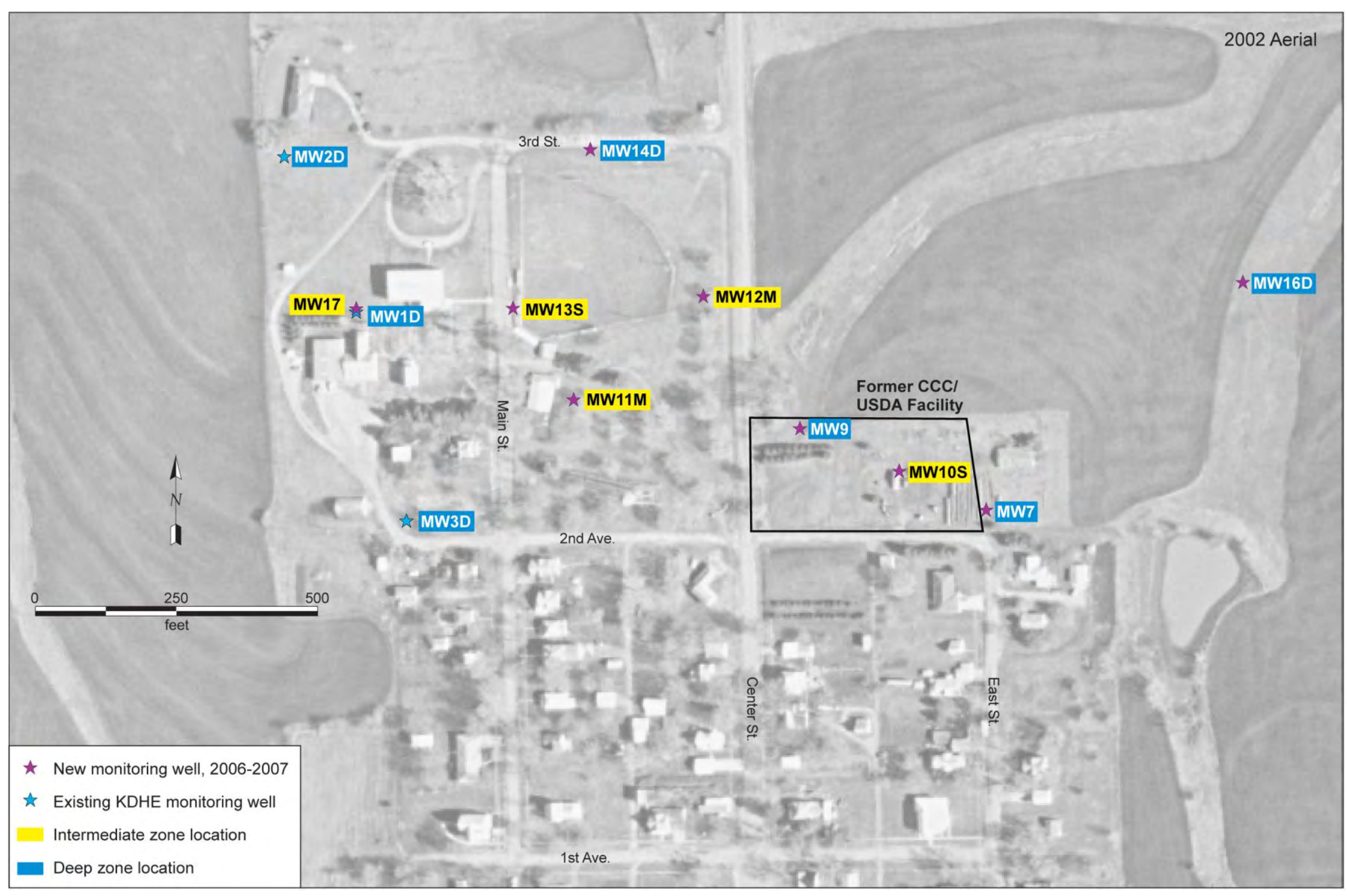

FIGURE 3.1 Wells equipped with data loggers for automatic water level monitoring in November 2011. Source of photograph: NAPP (2002). 


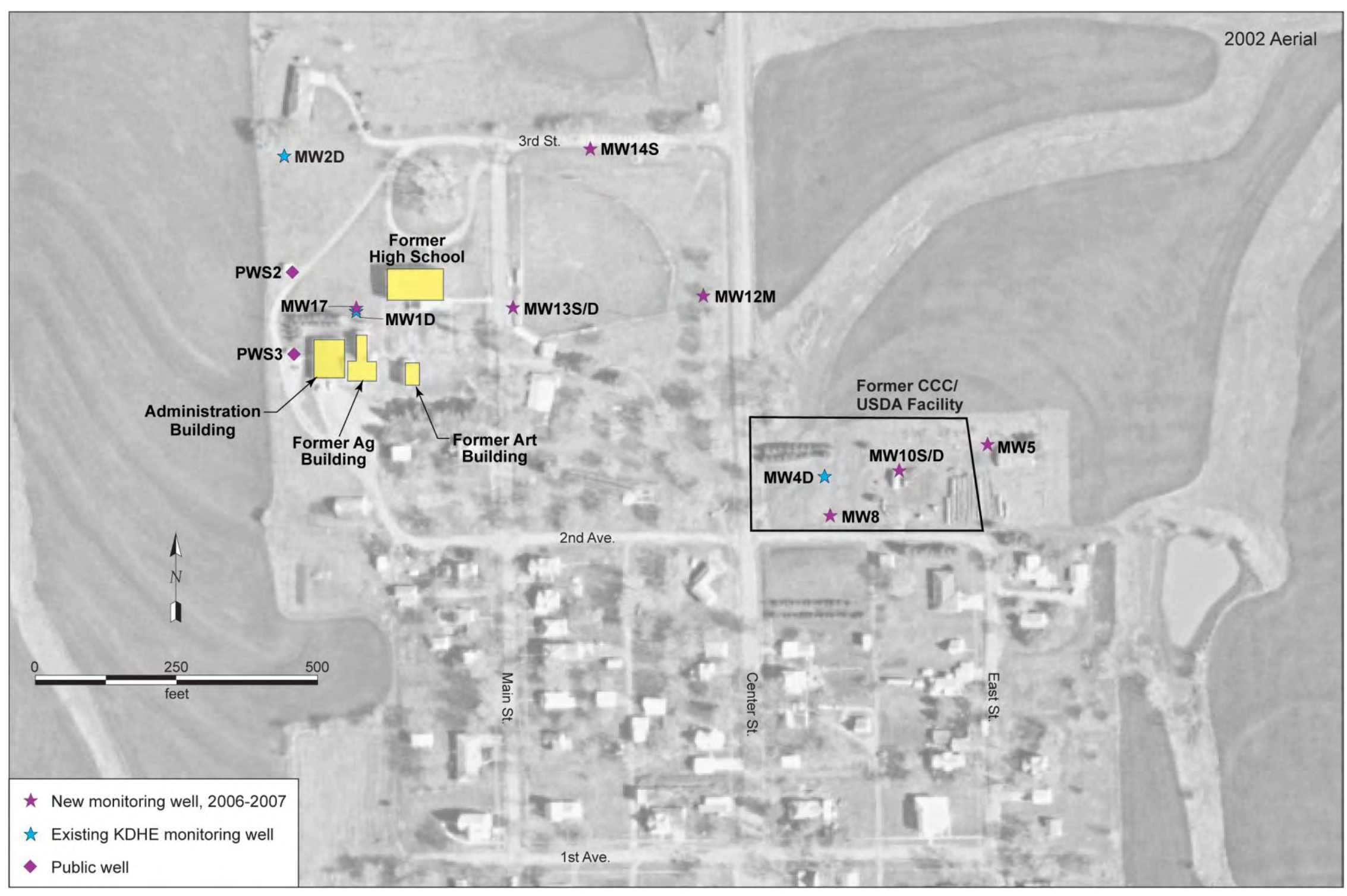

FIGURE 3.2 Groundwater sampling locations in September 2011. Source of photograph: NAPP (2002). 


\section{Results and Discussion}

\subsection{Groundwater Level Data}

The manual water level measurements taken during 2011 are in Table 4.1. Included are measurements made during sampling of the monitoring well network on September 28-29, 2011, and hand measurements taken on March 16 and November 18, 2011, for all wells in the automatic water level monitoring network. Evaluation of manual water levels measurements (together with the contaminant distribution data discussed in Section 4.2) continues to suggest that three vertically distinguishable aquifer zones are present at Barnes: shallow, intermediate, and deep. The designations "S", "M", and " $\mathrm{D}$ " in monitoring well names (Table 4.1) were assigned at the time of well installation to indicate shallow-, medium-, and deep-screened wells within individual well clusters, and they do not necessarily correspond with the designations of the aquifer zones identified later.

Table 4.2 shows the approximate water level elevations for wells screened in the three aquifer zones, as indicated by the long-term monitoring data accumulated since 2007 . As in prior monitoring events, three vertically distinguished aquifer zones are evident in the 2011 data.

Water level data collected by the automatic recorders, which are coincident in time at all monitored locations, provide the primary basis for determination of the topology of the potentiometric surface at any point in the cycles of groundwater pumping and recovery. The water levels measured by hand over a finite time period in the areally distributed network of monitoring wells do not capture the documented short-term, transient water level variations that are related to pumping of the public water supply wells. Figure 4.1 presents a potentiometric surface map (under non-pumping conditions) on November 18, 2011, for the network of wells in the deeper aquifer zone currently being monitored. For comparison, the maps in Figure 4.2a and Figure 4.2b were derived from measurements taken on February 2, 2010, under non-pumping and pumping conditions, respectively.

The hydrographs in Figure 4.3a and Figure 4.3b summarize data for the recording transducers in the deep-zone wells (January-November 2011). The hydrographs show that the groundwater levels in the deep zone declined steadily from January 1 to about mid-May, then rebounded very slightly or stabilized from May until late August, and finally declined again to the end of the data period. Over the entire period of automated monitoring, the water levels in the 
deep interval reached their transient (all-time) maximum in mid 2010. The decline observed through much of 2011 has returned the levels to elevations similar to those observed through 2008-2009, but the levels are still approximately $13 \mathrm{ft}$ higher than the all-time lowest levels observed in 2006. Although the data from the intermediate zone are comparatively limited, the available results follow the trends noted in the deep zone.

Throughout the monitoring at Barnes - until June 20, 2011 - the hydrographs consistently showed relatively large and frequent (typically once or twice per day) drawdown spikes associated with pumping of the public wells (Figure 4.4). After this date, the clear cycling pattern is no longer present. Although the hydrographs continue to exhibit the pumping effect of the public wells, more frequent pumping, primarily during daytime, is apparent. This change in the pumping pattern of the public supply wells reflects a recent upgrade of the public water system (Oentrich 2011). In the past, the public wells were pumped manually when the level in the distribution water tower became low. Starting in June 2011, pumping of the wells is activated by a sensor in response to demand, to keep the water level in the tower more constant. As part of the system upgrade, the distribution lines were replaced to reduce leakage. This improvement is likely to result in reduced pumping of the public wells.

\subsection{Analytical Results for Volatile Organic Compounds in Groundwater Samples and Lateral Distribution of the Contaminants}

The analytical data for VOCs in the groundwater samples collected in 2011 are in Table 4.3, together with data for the previous sampling events at Barnes. The highest concentration of carbon tetrachloride in sitewide monitoring continues to be found at intermediate-zone well MW10S (located in the eastern portion of the former CCC/USDA facility and screened at 93-103 ft BGL). In this well, carbon tetrachloride was detected at $37 \mu \mathrm{g} / \mathrm{L}$ in September 2011, down from $78 \mu \mathrm{g} / \mathrm{L}$ in September 2010.

The lateral distribution of carbon tetrachloride in groundwater in sampling events in September 2009, September 2010, and September 2011 is illustrated in Figure 4.5. The distribution in 2011 is similar to the previous distribution. Contaminant concentrations at and near the public water supply wells remained low to undetectable. Because of the pulsing influence on groundwater flow patterns when the public wells are pumping, no clear migration trend toward these wells has been evident in sitewide monitoring to date. Carbon tetrachloride was not detected in either public well during sampling in December 2011. 
The lateral distribution of chloroform in groundwater in 2011 (Figure 4.6) is also similar to the distribution during previous sampling events. The highest concentration of chloroform in sitewide sampling since 2007 has been found at well MW12M, located northwest of the former CCC/USDA facility and screened at 90-100 ft BGL (in the intermediate aquifer zone), with concentrations of 1.0-5.9 $\mu \mathrm{g} / \mathrm{L}$. Relatively lower dissolved oxygen and oxidation-reduction potential values have been measured consistently at this location (Table A.2 in Appendix A).

The vertical distribution of carbon tetrachloride in groundwater indicates that the highest concentrations are present in the intermediate zone, at wells MW10S (37 $\mathrm{g} / \mathrm{L})$, MW12M $(16 \mu \mathrm{g} / \mathrm{L})$, and MW13S (8.1 $\mu \mathrm{g} / \mathrm{L})$, as measured in September 2011 (Table 4.3). The deep-zone wells at these locations showed little to no change in carbon tetrachloride concentrations between the 2010 and 2011 sampling events (Figure 4.5). 
TABLE 4.1 Hand-measured water levels in 2011.

\begin{tabular}{|c|c|c|c|c|c|c|c|}
\hline \multirow[b]{2}{*}{ Well } & \multirow{2}{*}{$\begin{array}{l}\text { Reference } \\
\text { Elevation } \\
\text { (ft AMSL) }\end{array}$} & \multicolumn{2}{|c|}{ March 16, 2011} & \multicolumn{2}{|c|}{$\begin{array}{c}\text { September 28-29, } \\
2011\end{array}$} & \multicolumn{2}{|c|}{$\begin{array}{c}\text { November 18, } \\
2011 \\
\end{array}$} \\
\hline & & $\mathrm{ft} \mathrm{TOC}$ & $\mathrm{ft}$ AMSL & $\mathrm{ft}$ TOC & $\mathrm{ft}$ AMSL & $\mathrm{ft} \mathrm{TOC}$ & $\mathrm{ft}$ AMSL \\
\hline \multicolumn{8}{|c|}{ Shallow aquifer zone } \\
\hline MW1S & 1351.58 & - & - & - & - & - & - \\
\hline MW11S & 1336.58 & - & - & - & - & - & - \\
\hline MW12S & 1327.46 & - & - & - & - & - & - \\
\hline \multicolumn{8}{|c|}{ Intermediate aquifer zone } \\
\hline MW10S & 1331.33 & 72.18 & 1259.15 & 74.10 & 1257.23 & 75.11 & 1256.22 \\
\hline MW11M & 1336.51 & 77.13 & 1259.38 & - & - & 79.76 & 1256.75 \\
\hline MW12M & 1327.46 & 68.45 & 1259.01 & 71.25 & 1256.21 & 71.44 & 1256.02 \\
\hline MW13S & 1342.36 & - & - & 87.55 & 1254.81 & 88.15 & 1254.21 \\
\hline MW17 & 1351.77 & 94.11 & 1257.66 & 96.23 & 1255.54 & 97.38 & 1254.39 \\
\hline \multicolumn{8}{|c|}{ Deep aquifer zone } \\
\hline MW1D & 1351.33 & 113.67 & 1237.66 & 119.12 & 1232.21 & 120.28 & 1231.05 \\
\hline MW2D & 1348.85 & - & - & 116.22 & 1232.63 & 118.03 & 1230.82 \\
\hline MW3D & 1345.99 & 108.20 & 1237.79 & - & - & 114.66 & 1231.33 \\
\hline MW4D & 1326.32 & 89.84 & 1236.48 & 95.31 & 1231.01 & - & - \\
\hline MW5 & 1327.20 & - & - & 96.40 & 1230.80 & - & - \\
\hline MW6S & 1323.13 & - & - & - & - & - & - \\
\hline MW6D & 1323.15 & - & - & - & - & - & - \\
\hline MW7 & 1329.91 & 94.08 & 1235.83 & - & - & 99.74 & 1230.17 \\
\hline MW8 & 1330.06 & - & - & 98.42 & 1231.64 & - & - \\
\hline MW9 & 1321.86 & 85.45 & 1236.41 & - & - & 91.41 & 1230.45 \\
\hline MW10D & 1331.33 & - & - & 99.65 & 1231.68 & - & - \\
\hline MW11D & 1336.53 & - & - & - & - & - & - \\
\hline MW12D & 1327.52 & - & - & - & - & - & - \\
\hline MW13D & 1342.37 & - & - & 110.20 & 1232.17 & - & - \\
\hline MW14S & 1332.69 & - & - & 101.40 & 1231.29 & - & - \\
\hline MW14D & 1332.74 & 95.89 & 1236.85 & - & - & 103.91 & 1228.83 \\
\hline MW15S & 1309.34 & - & - & - & - & - & - \\
\hline MW15D & 1309.29 & 66.55 & 1242.74 & - & - & - & - \\
\hline MW16S & 1299.47 & - & - & - & - & - & - \\
\hline MW16D & 1299.52 & 65.18 & 1234.34 & - & - & 70.36 & 1229.16 \\
\hline Oentrich & 1336.93 & - & - & - & - & - & - \\
\hline
\end{tabular}


TABLE 4.2 Elevation ranges measured for the three aquifer zones, 2007-2011.

\begin{tabular}{lccc}
\hline & \multicolumn{3}{c}{ Elevation of Aquifer Zone (ft AMSL) } \\
\cline { 2 - 4 } Date & Shallow & Intermediate & Deep \\
\hline & & & \\
November 2011 & - & $1,254-1,257$ & $1,229-1,231$ \\
September 2011 & - & $1,255-1,257$ & $1,231-1,233$ \\
March 2011 & - & $1,258-1,259$ & $1,234-1,243$ \\
September 2010 & 1,312 & $1,263-1,265$ & $1,242-1,256$ \\
July 2010 & - & $1,267-1,269$ & $1,244-1,254$ \\
March-April 2010 & 1,315 & $1,257-1,259$ & $1,233-1,243$ \\
February 2010 & 1,311 & $1,255-1,257$ & $1,230-1,238$ \\
October 2009 & $1,275-1,307$ & $1,254-1,256$ & $1,229-1,237$ \\
June 2009 & $1,274-1,310$ & $1,255-1,258$ & $1,232-1,241$ \\
March 2009 & 1,308 & $1,251-1,256$ & $1,229-1,236$ \\
November 2008 & - & $1,257-1,259$ & $1,233-1,242$ \\
October 2008 & 1,314 & $1,256-1,259$ & $1,235-1,242$ \\
July 2008 & 1,312 & $1,255-1,258$ & $1,229-1,239$ \\
March 2008 & 1,309 & $1,250-1,254$ & $1,223-1,229$ \\
November 2007 & 1,307 & $1,249-1,254$ & $1,220-1,239$ \\
June 2007 & $1,276-1,314$ & $1,247-1,254$ & $1,221-1,228$ \\
& & & \\
\hline & & & \\
\hline
\end{tabular}


TABLE 4.3 Analytical results from the AGEM Laboratory for volatile organic compounds in groundwater samples, 2006-2011.

\begin{tabular}{|c|c|c|c|c|c|c|c|}
\hline \multirow[b]{2}{*}{ Location } & \multirow[b]{2}{*}{$\begin{array}{c}\text { Screen Interval } \\
\text { Depth } \\
\text { (ft BGL) }\end{array}$} & \multirow[b]{2}{*}{ Sample } & \multirow[b]{2}{*}{$\begin{array}{l}\text { Sample } \\
\text { Date }\end{array}$} & \multirow[b]{2}{*}{$\begin{array}{l}\text { Depth to } \\
\text { Water } \\
\text { (ft BTOC) }^{\mathrm{a}}\end{array}$} & \multicolumn{3}{|c|}{ Concentration $(\mu \mathrm{g} / \mathrm{L})$} \\
\hline & & & & & $\begin{array}{c}\text { Carbon } \\
\text { Tetrachloride }\end{array}$ & Chloroform & $\begin{array}{l}\text { Methyle } \\
\text { Chlori }\end{array}$ \\
\hline \multicolumn{8}{|c|}{ Previously existing monitoring wells } \\
\hline \multirow{11}{*}{ MW1S } & $13.3-23.3$ & Not sampled (well dry) & $7 / 19 / 06$ & - & - & - & - \\
\hline & & Not sampled (well dry) & $4 / 4 / 07$ & - & - & - & - \\
\hline & & Not sampled (well dry) & $11 / 18 / 07$ & - & - & - & - \\
\hline & & Not sampled (well dry) & $3 / 4 / 08$ & - & - & - & - \\
\hline & & Not sampled (well dry) & $7 / 9 / 08$ & - & - & - & - \\
\hline & & Not sampled (well dry) & $10 / 22 / 08$ & - & - & - & - \\
\hline & & Not sampled (well dry) & $3 / 4 / 09$ & - & - & - & - \\
\hline & & Not sampled (well dry) & $6 / 17 / 09$ & - & - & - & - \\
\hline & & Not sampled (well dry) & $9 / 30 / 09$ & - & _- & - & - \\
\hline & & Not sampled (well dry) & $3 / 31 / 10$ & - & - & - & - \\
\hline & & Not sampled (well dry) & $9 / 17 / 10$ & - & - & - & - \\
\hline \multirow[t]{13}{*}{ MW1D } & $139.85-159.4$ & BAMW1D-W-21688 & $7 / 19 / 06$ & 135.20 & 1.0 & $N D^{a}$ & ND \\
\hline & & BAMW1D-W-22565 & $4 / 4 / 07$ & 132.50 & 1.2 & ND & ND \\
\hline & & BAMW1D-W-22593 & $11 / 18 / 07$ & 124.89 & ND & ND & ND \\
\hline & & BAMW1D-W-22627 & $3 / 4 / 08$ & 127.66 & $0.2 \mathrm{~J}^{\mathrm{b}}$ & ND & ND \\
\hline & & BAMW1D-W-22668 & $7 / 9 / 08$ & 119.40 & $0.2 \mathrm{~J}$ & ND & ND \\
\hline & & BAMW1D-W-27720 & $10 / 22 / 08$ & 113.77 & ND & ND & ND \\
\hline & & BAMW1D-W-22703 & $3 / 4 / 09$ & 119.90 & ND & ND & ND \\
\hline & & BAMW1D-W-28639 & $6 / 17 / 09$ & 115.60 & ND & ND & ND \\
\hline & & BAMW1D-W-28678 & 9/30/09 & 120.40 & $0.3 \mathrm{~J}$ & ND & ND \\
\hline & & BAMW1D-W-28718 & $3 / 31 / 10$ & 114.55 & ND & ND & ND \\
\hline & & BAMW1D-W-28761 & $9 / 17 / 10$ & 105.28 & ND & ND & ND \\
\hline & & BAMW1D-W-28806 & $9 / 28 / 11$ & 119.12 & ND & ND & ND \\
\hline & & BAMW1DDUP-W-28820 & $9 / 28 / 11$ & - & ND & ND & ND \\
\hline \multirow[t]{12}{*}{ MW2D } & $133.26-152.93$ & BAMW2D-W-21687 & $7 / 19 / 06$ & 132.00 & ND & ND & ND \\
\hline & & BAMW2D-W-22564 & $4 / 4 / 07$ & 130.17 & ND & ND & ND \\
\hline & & BAMW2D-W-22594 & $11 / 18 / 07$ & 122.56 & ND & ND & ND \\
\hline & & BAMW2D-W-22628 & $3 / 7 / 08$ & 125.55 & ND & ND & ND \\
\hline & & BAMW2D-W-22669 & $7 / 10 / 08$ & 117.15 & ND & ND & ND \\
\hline & & BAMW2D-W-27721 & $10 / 22 / 08$ & 113.55 & ND & ND & ND \\
\hline & & BAMW2D-W-22704 & $3 / 4 / 09$ & 117.10 & ND & ND & ND \\
\hline & & BAMW2D-W-28640 & $6 / 18 / 09$ & 115.70 & ND & ND & ND \\
\hline & & BAMW2D-W-28679 & $9 / 30 / 09$ & 117.60 & ND & ND & ND \\
\hline & & BAMW2D-W-28719 & $3 / 31 / 10$ & 112.40 & ND & ND & ND \\
\hline & & BAMW2D-W-28762 & $9 / 17 / 10$ & 103.38 & ND & ND & ND \\
\hline & & BAMW2D-W-28807 & $9 / 28 / 11$ & 116.22 & ND & ND & ND \\
\hline \multirow[t]{11}{*}{ MW3D } & $133.02-152.73$ & BAMW3D-W-21686 & $7 / 19 / 06$ & 128.96 & ND & ND & ND \\
\hline & & BAMW3D-W-22567 & $4 / 4 / 07$ & 126.64 & ND & ND & ND \\
\hline & & BAMW3D-W-22595 & $11 / 19 / 07$ & 126.25 & ND & ND & ND \\
\hline & & BAMW3D-W-22629 & 3/7/08 & 121.90 & ND & ND & ND \\
\hline & & BAMW3D-W-22670 & $7 / 10 / 08$ & 113.30 & ND & ND & ND \\
\hline & & BAMW3D-W-27722 & $10 / 22 / 08$ & 108.50 & ND & ND & ND \\
\hline & & BAMW3D-W-22705 & $3 / 4 / 09$ & 116.10 & ND & ND & ND \\
\hline & & BAMW3D-W-28641 & $6 / 17 / 09$ & 110.15 & ND & ND & ND \\
\hline & & BAMW3D-W-28680 & $9 / 30 / 09$ & 116.30 & ND & ND & ND \\
\hline & & BAMW3D-W-28720 & $4 / 1 / 10$ & 108.86 & ND & ND & ND \\
\hline & & BAMW3D-W-28763 & $9 / 17 / 10$ & 99.92 & ND & ND & ND \\
\hline \multirow{5}{*}{ MW4D } & $98.38-118.22$ & BAMW4D-W-21690 & $7 / 20 / 06$ & 108.80 & 2.1 & ND & ND \\
\hline & & BAMW4D-W-22583 & $4 / 6 / 07$ & 108.00 & 3.5 & $0.1 \mathrm{~J}$ & ND \\
\hline & & BAMW4D-W-22596 & $11 / 19 / 07$ & 101.39 & 1.7 & $0.4 \mathrm{~J}$ & ND \\
\hline & & BAMW4D-W-22642 & $3 / 9 / 08$ & 101.74 & 18 & $0.4 \mathrm{~J}$ & ND \\
\hline & & BAMW4D-W-22671 & $7 / 12 / 08$ & 93.60 & 9.4 & $0.5 \mathrm{~J}$ & ND \\
\hline
\end{tabular}


TABLE 4.3 (Cont.)

\begin{tabular}{|c|c|c|c|c|c|c|c|}
\hline \multirow[b]{2}{*}{ Location } & \multirow[b]{2}{*}{$\begin{array}{c}\text { Screen Interval } \\
\text { Depth } \\
\text { (ft BGL) }\end{array}$} & \multirow[b]{2}{*}{ Sample } & \multirow[b]{2}{*}{$\begin{array}{l}\text { Sample } \\
\text { Date }\end{array}$} & \multirow[b]{2}{*}{$\begin{array}{l}\text { Depth to } \\
\text { Water } \\
(\mathrm{ft} \text { BTOC) }\end{array}$} & \multicolumn{3}{|c|}{ Concentration $(\mu \mathrm{g} / \mathrm{L})$} \\
\hline & & & & & $\begin{array}{c}\text { Carbon } \\
\text { Tetrachloride }\end{array}$ & Chloroform & $\begin{array}{l}\text { Methylene } \\
\text { Chloride }\end{array}$ \\
\hline \multicolumn{8}{|c|}{ Previously existing monitoring wells (cont.) } \\
\hline MW4D & $98.38-118.22$ & $\begin{array}{l}\text { BAMW4D-W-27723 } \\
\text { BAMW4D-W-22706 } \\
\text { BAMW4D-W-28642 } \\
\text { BAMW4D-W-28681 } \\
\text { BAMW4D-W-28721 } \\
\text { BAMW4D-W-28764 } \\
\text { BAMW4D-W-28808 }\end{array}$ & $\begin{array}{r}10 / 23 / 08 \\
3 / 5 / 09 \\
6 / 18 / 09 \\
9 / 30 / 09 \\
3 / 31 / 10 \\
9 / 17 / 10 \\
9 / 28 / 11\end{array}$ & $\begin{array}{l}89.90 \\
94.75 \\
92.25 \\
95.70 \\
91.45 \\
81.25 \\
95.31\end{array}$ & $\begin{array}{c}7.6 \\
7.2 \\
9.1 \\
13 \\
13 \\
12 \\
10\end{array}$ & $\begin{array}{l}\text { ND } \\
0.3 \mathrm{~J} \\
\text { ND } \\
0.3 \mathrm{~J} \\
0.4 \mathrm{~J} \\
\text { ND } \\
\text { ND }\end{array}$ & $\begin{array}{l}\text { ND } \\
\text { ND } \\
\text { ND } \\
\text { ND } \\
\text { ND } \\
\text { ND } \\
\text { ND }\end{array}$ \\
\hline \multicolumn{8}{|c|}{ CCC/USDA wells installed during the 2006-2007 investigation } \\
\hline MW5 & $110-120$ & $\begin{array}{l}\text { BAMW5-W-22589 } \\
\text { BAMW5-W-22597 } \\
\text { BAMW5-W-22637 } \\
\text { BAMW5-W-22672 } \\
\text { BAMW5-W-27724 } \\
\text { BAMW5-W-22707 } \\
\text { BAMW5-W-28643 } \\
\text { BAMW5-W-28682 } \\
\text { BAMW5-W-28722 } \\
\text { BAMW5-W-28765 } \\
\text { BAMW5-W-28809 }\end{array}$ & $\begin{array}{r}4 / 6 / 07 \\
11 / 19 / 07 \\
3 / 8 / 08 \\
7 / 11 / 08 \\
10 / 23 / 08 \\
3 / 5 / 09 \\
6 / 19 / 09 \\
9 / 30 / 09 \\
3 / 30 / 10 \\
9 / 17 / 10 \\
9 / 28 / 11\end{array}$ & $\begin{array}{r}108.40 \\
102.78 \\
102.00 \\
93.80 \\
91.40 \\
96.90 \\
93.80 \\
96.60 \\
92.06 \\
83.10 \\
96.40\end{array}$ & $\begin{array}{l}0.6 \mathrm{~J} \\
0.6 \mathrm{~J} \\
0.7 \mathrm{~J} \\
\mathrm{ND} \\
3.0 \\
3.2 \\
4.8 \\
7.2 \\
7.7 \\
11 \\
10\end{array}$ & $\begin{array}{l}\text { ND } \\
\text { ND } \\
\text { ND } \\
\text { ND } \\
\text { ND } \\
\text { ND } \\
\text { ND } \\
\text { ND } \\
0.3 \mathrm{~J} \\
\text { ND } \\
\text { ND }\end{array}$ & $\begin{array}{l}\text { ND } \\
\text { ND } \\
\text { ND } \\
\text { ND } \\
\text { ND } \\
\text { ND } \\
\text { ND } \\
\text { ND } \\
\text { ND } \\
\text { ND } \\
\text { ND }\end{array}$ \\
\hline MW6S & $90.5-100.5$ & $\begin{array}{l}\text { Not sampled (well dry) } \\
\text { BAMW6S-W-22598 } \\
\text { BAMW6S-W-22635 } \\
\text { BAMW6S-W-22673 } \\
\text { BAMW6S-W-27725 } \\
\text { BAMW6S-W-22708 } \\
\text { BAMW6S-W-28644 } \\
\text { BAMW6S-W-28683 } \\
\text { BAMW6S-W-28723 } \\
\text { BAMW6S-W-28766 }\end{array}$ & $\begin{array}{r}4 / 4 / 07 \\
11 / 19 / 07 \\
3 / 8 / 08 \\
7 / 11 / 08 \\
10 / 23 / 08 \\
3 / 5 / 09 \\
6 / 18 / 09 \\
10 / 1 / 09 \\
3 / 31 / 10 \\
9 / 18 / 10\end{array}$ & $\begin{array}{l}- \\
96.10 \\
94.50 \\
88.10 \\
84.60 \\
87.00 \\
86.05 \\
88.85 \\
86.15 \\
76.46\end{array}$ & $\begin{array}{l}- \\
0.3 \mathrm{~J} \\
0.4 \mathrm{~J} \\
N D \\
N D \\
N D \\
N D \\
N D \\
0.4 \mathrm{~J} \\
N D\end{array}$ & $\begin{array}{l}- \\
\text { ND } \\
\text { ND } \\
\text { ND } \\
\text { ND } \\
\text { ND } \\
\text { ND } \\
\text { ND } \\
\text { ND } \\
\text { ND }\end{array}$ & $\begin{array}{l}- \\
\text { ND } \\
\text { ND } \\
\text { ND } \\
\text { ND } \\
\text { ND } \\
\text { ND } \\
\text { ND } \\
\text { ND } \\
\text { ND }\end{array}$ \\
\hline MW6D & $105-115$ & $\begin{array}{l}\text { BAMW6D-W-22573 } \\
\text { BAMW6D-W-22599 } \\
\text { BAMW6D-W-22636 } \\
\text { BAMW6D-W-22674 } \\
\text { BAMW6D-W-27726 } \\
\text { BAMW6D-W-22709 } \\
\text { BAMW6D-W-28645 } \\
\text { BAMW6D-W-28684 } \\
\text { BAMW6D-W-28724 } \\
\text { BAMW6D-W-28767 }\end{array}$ & $\begin{array}{r}4 / 5 / 07 \\
11 / 19 / 07 \\
3 / 8 / 08 \\
7 / 11 / 08 \\
10 / 23 / 08 \\
3 / 5 / 09 \\
6 / 18 / 09 \\
10 / 1 / 09 \\
3 / 31 / 10 \\
9 / 18 / 10\end{array}$ & $\begin{array}{r}105.00 \\
98.50 \\
98.50 \\
89.50 \\
87.15 \\
93.00 \\
88.70 \\
91.92 \\
87.84 \\
79.35\end{array}$ & $\begin{array}{l}\text { ND } \\
0.5 \mathrm{~J} \\
0.8 \mathrm{~J} \\
0.9 \mathrm{~J} \\
1.1 \\
1.4 \\
1.5 \\
1.5 \\
1.2 \\
2.0\end{array}$ & $\begin{array}{l}\text { ND } \\
\text { ND } \\
\text { ND } \\
\text { ND } \\
\text { ND } \\
\text { ND } \\
\text { ND } \\
\text { ND } \\
\text { ND } \\
\text { ND }\end{array}$ & $\begin{array}{l}\text { ND } \\
\text { ND } \\
\text { ND } \\
\text { ND } \\
\text { ND } \\
\text { ND } \\
\text { ND } \\
\text { ND } \\
\text { ND } \\
\text { ND }\end{array}$ \\
\hline MW7 & $116-126$ & $\begin{array}{l}\text { BAMW7-W-22588 } \\
\text { BAMW7-W-22600 } \\
\text { BAMW7-W-22643 } \\
\text { BAMW7-W-222675 } \\
\text { BAMW7-W-27727 } \\
\text { BAMW7-W-22710 } \\
\text { BAMW7-W-28646 } \\
\text { BAMW7-W-28685 } \\
\text { BAMW7-W-28725 } \\
\text { BAMW7-W-28768 }\end{array}$ & $\begin{array}{r}4 / 6 / 07 \\
11 / 19 / 07 \\
3 / 9 / 08 \\
7 / 12 / 08 \\
10 / 23 / 08 \\
3 / 5 / 09 \\
6 / 19 / 09 \\
9 / 30 / 09 \\
3 / 30 / 10 \\
9 / 17 / 10\end{array}$ & $\begin{array}{r}111.11 \\
105.50 \\
105.62 \\
97.50 \\
94.90 \\
99.80 \\
95.75 \\
99.55 \\
94.56 \\
85.67\end{array}$ & $\begin{array}{l}1.0 \\
2.6 \\
2.8 \\
1.7 \\
2.1 \\
1.4 \\
1.4 \\
1.6 \\
1.6 \\
2.6\end{array}$ & $\begin{array}{l}\text { ND } \\
\text { ND } \\
\text { ND } \\
\text { ND } \\
\text { ND } \\
\text { ND } \\
\text { ND } \\
\text { ND } \\
\text { ND } \\
\text { ND }\end{array}$ & $\begin{array}{l}\text { ND } \\
\text { ND } \\
\text { ND } \\
\text { ND } \\
\text { ND } \\
\text { ND } \\
\text { ND } \\
\text { ND } \\
\text { ND } \\
\text { ND }\end{array}$ \\
\hline
\end{tabular}


TABLE 4.3 (Cont.)

\begin{tabular}{|c|c|c|c|c|c|c|c|}
\hline \multirow[b]{2}{*}{ Location } & \multirow[b]{2}{*}{$\begin{array}{c}\text { Screen Interval } \\
\text { Depth } \\
\text { (ft BGL) }\end{array}$} & \multirow[b]{2}{*}{ Sample } & \multirow[b]{2}{*}{$\begin{array}{l}\text { Sample } \\
\text { Date }\end{array}$} & \multirow[b]{2}{*}{$\begin{array}{l}\text { Depth to } \\
\text { Water } \\
\text { (ft BTOC) }\end{array}$} & \multicolumn{3}{|c|}{ Concentration $(\mu \mathrm{g} / \mathrm{L})$} \\
\hline & & & & & $\begin{array}{c}\text { Carbon } \\
\text { Tetrachloride }\end{array}$ & Chloroform & $\begin{array}{r}\text { Methyl } \\
\text { Chlor }\end{array}$ \\
\hline \multicolumn{8}{|c|}{ CCC/USDA wells installed during the 2006-2007 investigation (cont.) } \\
\hline \multirow[t]{11}{*}{ MW8 } & $110-120$ & BAMW8-W-22584 & $4 / 6 / 07$ & 111.71 & 14 & $0.7 \mathrm{~J}$ & ND \\
\hline & & BAMW8-W-22601 & $11 / 19 / 07$ & 105.17 & 23 & $0.6 \mathrm{~J}$ & ND \\
\hline & & BAMW8-W-22652 & $3 / 10 / 08$ & 104.38 & 19 & $0.6 \mathrm{~J}$ & ND \\
\hline & & BAMW8-W-22676 & $7 / 11 / 08$ & 95.75 & 21 & $0.6 \mathrm{~J}$ & ND \\
\hline & & BAMW8-W-27728 & $10 / 23 / 08$ & 93.40 & 24 & 1.0 & ND \\
\hline & & BAMW8-W-22711 & $3 / 5 / 09$ & 98.60 & 20 & 1.3 & ND \\
\hline & & BAMW8-W-28647 & $6 / 19 / 09$ & 95.00 & 26 & 1.7 & ND \\
\hline & & BAMW8-W-28686 & $9 / 30 / 09$ & 99.20 & 29 & 2.2 & ND \\
\hline & & BAMW8-W-28726 & $3 / 31 / 10$ & 94.06 & 30 & 2.0 & ND \\
\hline & & BAMW8-W-28769 & $9 / 17 / 10$ & 84.95 & 31 & 2.1 & ND \\
\hline & & BAMW8-W-28810 & $9 / 29 / 11$ & 98.42 & 19 & 1.6 & ND \\
\hline \multirow{10}{*}{ MW9 } & $100-110$ & BAMW9-W-22582 & $4 / 5 / 07$ & 102.90 & 1.0 & ND & ND \\
\hline & & BAMW9-W-22602 & $11 / 19 / 07$ & 97.20 & 7.7 & $0.6 \mathrm{~J}$ & ND \\
\hline & & BAMW9-W-22647 & $3 / 9 / 08$ & 96.40 & 3.0 & $0.3 \mathrm{~J}$ & ND \\
\hline & & BAMW9-W-22678 & $7 / 11 / 08$ & 87.65 & 1.3 & $0.3 \mathrm{~J}$ & ND \\
\hline & & BAMW9-W-27729 & $10 / 24 / 08$ & 86.60 & 2.2 & $0.2 \mathrm{~J}$ & ND \\
\hline & & BAMW9-W-22712 & $3 / 5 / 09$ & 90.40 & 2.3 & ND & ND \\
\hline & & BAMW9-W-28648 & $6 / 17 / 09$ & 83.16 & 1.1 & ND & ND \\
\hline & & BAMW9-W-28687 & 9/29/09 & 87.10 & 4.6 & ND & ND \\
\hline & & BAMW9-W-28727 & $3 / 31 / 10$ & 82.45 & 2.9 & ND & ND \\
\hline & & BAMW9-W-28770 & $9 / 18 / 10$ & 75.46 & 1.4 & ND & ND \\
\hline \multirow[t]{11}{*}{ MW10S } & $93-103$ & BAMW10S-W-22586 & $4 / 6 / 07$ & 82.55 & 20 & 1.4 & ND \\
\hline & & BAMW10S-W-22603 & $11 / 19 / 07$ & 77.81 & 11 & $0.7 \mathrm{~J}$ & ND \\
\hline & & BAMW10S-W-22649 & $3 / 10 / 08$ & 77.47 & 56 & 2.0 & ND \\
\hline & & BAMW10S-W-22679 & $7 / 11 / 08$ & 73.40 & 49 & 1.8 & ND \\
\hline & & BAMW10S-W-27730 & $10 / 23 / 08$ & 72.00 & 68 & 2.3 & ND \\
\hline & & BAMW10S-W-22713 & $3 / 5 / 09$ & 76.00 & 49 & 2.1 & ND \\
\hline & & BAMW10S-W-28649 & $6 / 19 / 09$ & 73.40 & 76 & 2.5 & ND \\
\hline & & BAMW10S-W-28688 & $9 / 30 / 09$ & 75.65 & 53 & 2.4 & ND \\
\hline & & BAMW10S-W-28728 & $3 / 30 / 10$ & 71.96 & 73 & 3.0 & ND \\
\hline & & BAMW10S-W-28771 & $9 / 17 / 10$ & 65.95 & 78 & 2.9 & ND \\
\hline & & BAMW10S-W-28811 & $9 / 28 / 11$ & 74.10 & 37 & 2.1 & ND \\
\hline \multirow[t]{11}{*}{ MW10D } & $115-125$ & BAMW10D-W-22585 & $4 / 6 / 07$ & 113.14 & 2.4 & $0.2 \mathrm{~J}$ & ND \\
\hline & & BAMW10D-W-22604 & $11 / 19 / 07$ & 106.22 & 6.3 & $0.5 \mathrm{~J}$ & ND \\
\hline & & BAMW10D-W-22646 & $3 / 9 / 08$ & 106.36 & 5.7 & $0.5 \mathrm{~J}$ & ND \\
\hline & & BAMW10D-W-22680 & $7 / 11 / 08$ & 97.30 & 3.9 & $0.7 \mathrm{~J}$ & ND \\
\hline & & BAMW10D-W-27731 & $10 / 23 / 08$ & 95.00 & 4.4 & $0.6 \mathrm{~J}$ & ND \\
\hline & & BAMW10D-W-22714 & $3 / 5 / 09$ & 101.30 & 5.3 & $0.4 \mathrm{~J}$ & ND \\
\hline & & BAMW10D-W-28650 & $6 / 19 / 09$ & 96.75 & 4.8 & $0.6 \mathrm{~J}$ & ND \\
\hline & & BAMW10D-W-28689 & 9/30/09 & 100.45 & 4.3 & $0.4 \mathrm{~J}$ & ND \\
\hline & & BAMW10D-W-28729 & $3 / 30 / 10$ & 96.86 & 4.4 & $0.4 \mathrm{~J}$ & ND \\
\hline & & BAMW10D-W-28772 & $9 / 17 / 10$ & 86.92 & 4.8 & ND & ND \\
\hline & & BAMW10D-W-28812 & $9 / 28 / 11$ & 99.65 & 3.3 & $0.3 \mathrm{~J}$ & ND \\
\hline \multirow[t]{10}{*}{ MW11S } & $40-50$ & BAMW11S-W-22570 & $4 / 4 / 07$ & 25.90 & ND & 1.1 & ND \\
\hline & & BAMW11S-W-22605 & $11 / 19 / 07$ & 29.20 & ND & $0.6 \mathrm{~J}$ & ND \\
\hline & & BAMW11S-W-22630 & $3 / 5 / 08$ & 27.70 & ND & $0.6 \mathrm{~J}$ & ND \\
\hline & & BAMW11S-W-22681 & $7 / 10 / 08$ & 24.80 & ND & $0.4 \mathrm{~J}$ & ND \\
\hline & & BAMW11S-W-27732 & $10 / 23 / 08$ & 22.50 & ND & $0.3 \mathrm{~J}$ & ND \\
\hline & & BAMW11S-W-22715 & $3 / 4 / 09$ & 28.00 & ND & ND & ND \\
\hline & & BAMW11S-W-28651 & $6 / 19 / 09$ & 26.64 & ND & ND & ND \\
\hline & & BAMW11S-W-28690 & $10 / 1 / 09$ & 29.50 & ND & ND & ND \\
\hline & & BAMW11S-W-28730 & $3 / 31 / 10$ & 21.50 & ND & ND & ND \\
\hline & & BAMW11S-W-28773 & $9 / 18 / 10$ & 24.71 & ND & ND & ND \\
\hline
\end{tabular}


TABLE 4.3 (Cont.)

\begin{tabular}{|c|c|c|c|c|c|c|c|}
\hline \multirow[b]{2}{*}{ Location } & \multirow[b]{2}{*}{$\begin{array}{c}\text { Screen Interval } \\
\text { Depth } \\
\text { (ft BGL) }\end{array}$} & \multirow[b]{2}{*}{ Sample } & \multirow[b]{2}{*}{$\begin{array}{l}\text { Sample } \\
\text { Date }\end{array}$} & \multirow[b]{2}{*}{$\begin{array}{l}\text { Depth to } \\
\text { Water } \\
(\mathrm{ft} \mathrm{BTOC})^{\mathrm{a}}\end{array}$} & \multicolumn{3}{|c|}{ Concentration $(\mu \mathrm{g} / \mathrm{L})$} \\
\hline & & & & & $\begin{array}{c}\text { Carbon } \\
\text { Tetrachloride }\end{array}$ & Chloroform & $\begin{array}{l}\text { Methyl } \\
\text { Chlori }\end{array}$ \\
\hline \multicolumn{8}{|c|}{ CCC/USDA wells installed during the 2006-2007 investigation (cont.) } \\
\hline \multirow[t]{10}{*}{ MW11M } & $90-100$ & BAMW11M-W-22572 & $4 / 5 / 07$ & 89.30 & ND & ND & ND \\
\hline & & BAMW11M-W-22606 & $11 / 19 / 07$ & 82.33 & 3.7 & ND & ND \\
\hline & & BAMW11M-W-22644 & $3 / 6 / 08$ & 82.65 & 2.4 & $0.5 \mathrm{~J}$ & ND \\
\hline & & BAMW11M-W-22682 & $7 / 10 / 08$ & 78.85 & 2.4 & $0.7 \mathrm{~J}$ & ND \\
\hline & & BAMW11M-W-27733 & $10 / 23 / 08$ & 77.80 & 1.7 & 2.1 & ND \\
\hline & & BAMW11M-W-22716 & $3 / 4 / 09$ & 80.30 & $0.6 \mathrm{~J}$ & 1.2 & ND \\
\hline & & BAMW11M-W-28652 & $6 / 19 / 09$ & 78.90 & ND & 1.1 & ND \\
\hline & & BAMW11M-W-28691 & $10 / 1 / 09$ & 80.45 & ND & $0.5 \mathrm{~J}$ & ND \\
\hline & & BAMW11M-W-28731 & $3 / 31 / 10$ & 77.90 & $0.5 \mathrm{~J}$ & $0.8 \mathrm{~J}$ & ND \\
\hline & & BAMW11M-W-28774 & $9 / 18 / 10$ & 71.22 & ND & ND & ND \\
\hline \multirow[t]{10}{*}{ MW11D } & $125-135$ & BAMW11D-W-22571 & $4 / 4 / 07$ & 117.15 & 1.1 & ND & ND \\
\hline & & BAMW11D-W-22607 & $11 / 19 / 07$ & 112.46 & $0.8 \mathrm{~J}$ & ND & ND \\
\hline & & BAMW11D-W-22639 & $3 / 5 / 08$ & 110.50 & $0.4 \mathrm{~J}$ & ND & ND \\
\hline & & BAMW11D-W-22683 & $7 / 10 / 08$ & 102.10 & $0.9 \mathrm{~J}$ & ND & ND \\
\hline & & BAMW11D-W-27734 & $10 / 23 / 08$ & 101.03 & $0.9 \mathrm{~J}$ & $0.2 \mathrm{~J}$ & ND \\
\hline & & BAMW11D-W-22717 & $3 / 4 / 09$ & 105.03 & $0.8 \mathrm{~J}$ & ND & ND \\
\hline & & BAMW11D-W-28653 & $6 / 19 / 09$ & 100.90 & ND & ND & ND \\
\hline & & BAMW11D-W-28692 & 10/1/09 & 105.95 & 1.0 & ND & ND \\
\hline & & BAMW11D-W-28732 & $4 / 1 / 10$ & 100.10 & $0.5 \mathrm{~J}$ & ND & ND \\
\hline & & BAMW11D-W-28775 & $9 / 18 / 10$ & 90.97 & ND & ND & ND \\
\hline \multirow[t]{10}{*}{ MW12S } & $43-50$ & Not sampled (well dry) & $4 / 5 / 07$ & - & - & - & - \\
\hline & & Not sampled (well dry) & $11 / 19 / 07$ & - & - & - & - \\
\hline & & Not sampled (well dry) & $3 / 10 / 08$ & - & - & - & - \\
\hline & & Not sampled (well dry) & $7 / 10 / 08$ & - & - & - & - \\
\hline & & Not sampled (well dry) & $10 / 22 / 08$ & - & - & - & - \\
\hline & & Not sampled (well dry) & $3 / 4 / 09$ & - & - & - & - \\
\hline & & BAMW12S-W-28654 & $6 / 19 / 09$ & - & ND & ND & ND \\
\hline & & Not sampled (well dry) & 10/1/09 & - & - & - & - \\
\hline & & Not sampled (well dry) & $3 / 31 / 10$ & - & - & - & - \\
\hline & & Not sampled (well dry) & $9 / 18 / 10$ & - & - & - & - \\
\hline \multirow[t]{12}{*}{ MW12M } & $90-100$ & BAMW12M-W-22580 & $4 / 5 / 07$ & 81.05 & 20 & 4.2 & ND \\
\hline & & BAMW12M-W-22609 & $11 / 19 / 07$ & 74.50 & 18 & 5.1 & ND \\
\hline & & BAMW12M-W-22651 & $3 / 10 / 08$ & 74.77 & 18 & 2.6 & ND \\
\hline & & BAMW12M-W-22685 & $7 / 10 / 08$ & 70.10 & 27 & 4.2 & ND \\
\hline & & BAMW12M-W-27736 & $10 / 22 / 08$ & 69.72 & 18 & 4.5 & ND \\
\hline & & BAMW12M-W-22719 & $3 / 4 / 09$ & 76.50 & 25 & 4.4 & ND \\
\hline & & BAMW12M-W-28655 & $6 / 19 / 09$ & 70.05 & 28 & 4.9 & ND \\
\hline & & BAMW12M-W-28694 & 10/1/09 & 72.90 & 26 & 5.1 & ND \\
\hline & & BAMW12M-W-28734 & $3 / 31 / 10$ & 70.45 & 2.2 & 1.0 & ND \\
\hline & & BAMW12M-W-28777 & $9 / 18 / 10$ & 63.90 & 6.6 & 5.9 & ND \\
\hline & & BAMW12M-W-28813 & $9 / 28 / 11$ & 71.25 & 16 & 5.1 & ND \\
\hline & & BAMW12MDUP-W-28821 & $9 / 28 / 11$ & - & 17 & 5.2 & ND \\
\hline \multirow[t]{10}{*}{ MW12D } & $115-125$ & BAMW12D-W-22576 & $4 / 5 / 07$ & 110.20 & $0.6 \mathrm{~J}$ & ND & ND \\
\hline & & BAMW12D-W-22610 & $11 / 18 / 07$ & 102.00 & 1.6 & ND & ND \\
\hline & & BAMW12D-W-22641 & $3 / 9 / 08$ & 103.30 & 1.0 & ND & ND \\
\hline & & BAMW12D-W-22686 & $7 / 11 / 08$ & 93.70 & $0.7 \mathrm{~J}$ & ND & ND \\
\hline & & BAMW12D-W-27737 & $10 / 22 / 08$ & 91.12 & $0.9 \mathrm{~J}$ & ND & ND \\
\hline & & BAMW12D-W-22757 & $3 / 4 / 09$ & 96.80 & $0.7 \mathrm{~J}$ & ND & ND \\
\hline & & BAMW12D-W-28656 & $6 / 19 / 09$ & 93.65 & ND & ND & ND \\
\hline & & BAMW12D-W-28695 & $10 / 1 / 09$ & 96.90 & 1.5 & ND & ND \\
\hline & & BAMW12D-W-28735 & $3 / 31 / 10$ & 93.55 & 1.0 & $0.2 \mathrm{~J}$ & ND \\
\hline & & BAMW12D-W-28778 & $9 / 18 / 10$ & 83.10 & $0.6 \mathrm{~J}$ & ND & ND \\
\hline
\end{tabular}


TABLE 4.3 (Cont.)

\begin{tabular}{|c|c|c|c|c|c|c|c|}
\hline \multirow[b]{2}{*}{ Location } & \multirow[b]{2}{*}{$\begin{array}{c}\text { Screen Interval } \\
\text { Depth } \\
\text { (ft BGL) }\end{array}$} & \multirow[b]{2}{*}{ Sample } & \multirow[b]{2}{*}{$\begin{array}{l}\text { Sample } \\
\text { Date }\end{array}$} & \multirow[b]{2}{*}{$\begin{array}{l}\text { Depth to } \\
\text { Water } \\
\text { (ft BTOC) }\end{array}$} & \multicolumn{3}{|c|}{ Concentration $(\mu \mathrm{g} / \mathrm{L})$} \\
\hline & & & & & $\begin{array}{c}\text { Carbon } \\
\text { Tetrachloride }\end{array}$ & Chloroform & $\begin{array}{l}\text { Methy } \\
\text { Chlo }\end{array}$ \\
\hline \multicolumn{8}{|c|}{ CCC/USDA wells installed during the 2006-2007 investigation (cont.) } \\
\hline \multirow[t]{11}{*}{ MW13S } & $112-122$ & BAMW13S-W-22575 & $4 / 5 / 07$ & 101.00 & 21 & 1.6 & ND \\
\hline & & BAMW13S-W-22611 & $11 / 19 / 07$ & 92.23 & 17 & 1.8 & ND \\
\hline & & BAMW13S-W-22650 & $3 / 10 / 08$ & 92.10 & 17 & 1.5 & ND \\
\hline & & BAMW13S-W-22687 & $7 / 9 / 08$ & 87.00 & 17 & 1.9 & ND \\
\hline & & BAMW13S-W-27738 & $10 / 22 / 08$ & 86.00 & 20 & 1.6 & ND \\
\hline & & BAMW13S-W-22758 & $3 / 4 / 09$ & 88.75 & 14 & 1.1 & ND \\
\hline & & BAMW13S-W-28657 & $6 / 18 / 09$ & 86.85 & 16 & 1.1 & ND \\
\hline & & BAMW13S-W-28696 & 9/30/09 & 88.45 & 12 & $0.9 \mathrm{~J}$ & ND \\
\hline & & BAMW13S-W-28736 & $4 / 1 / 10$ & 85.65 & 13 & $0.8 \mathrm{~J}$ & ND \\
\hline & & BAMW13S-W-28779 & $9 / 18 / 10$ & 78.01 & 6.2 & 1.2 & ND \\
\hline & & BAMW13S-W-28814 & $9 / 28 / 11$ & 87.55 & 8.1 & $0.7 \mathrm{~J}$ & ND \\
\hline \multirow[t]{11}{*}{ MW13D } & $127-137$ & BAMW13D-W-22574 & $4 / 5 / 07$ & 124.67 & 3.5 & $0.4 \mathrm{~J}$ & ND \\
\hline & & BAMW13D-W-22612 & $11 / 19 / 07$ & 117.83 & 5.9 & $0.2 \mathrm{~J}$ & ND \\
\hline & & BAMW13D-W-22645 & $3 / 9 / 08$ & 118.19 & 11 & 1.1 & ND \\
\hline & & BAMW13D-W-22688 & $7 / 9 / 08$ & 107.90 & 5.9 & $0.9 \mathrm{~J}$ & ND \\
\hline & & BAMW13D-W-27739 & 10/22/08 & 105.30 & 6.6 & $0.6 \mathrm{~J}$ & ND \\
\hline & & BAMW13D-W-22759 & $3 / 4 / 09$ & 110.58 & 5.9 & $0.6 \mathrm{~J}$ & ND \\
\hline & & BAMW13D-W-28658 & 6/18/09 & 106.80 & 6.2 & ND & ND \\
\hline & & BAMW13D-W-28697 & 9/30/09 & 112.85 & 7.2 & 1.0 & ND \\
\hline & & BAMW13D-W-28737 & $4 / 1 / 10$ & 105.75 & 5.5 & $0.5 \mathrm{~J}$ & ND \\
\hline & & BAMW13D-W-28780 & 9/18/10 & 96.88 & 5.8 & $0.6 \mathrm{~J}$ & ND \\
\hline & & BAMW13D-W-28815 & $9 / 28 / 11$ & 110.20 & 2.4 & ND & ND \\
\hline \multirow[t]{11}{*}{ MW14S } & $108-118$ & BAMW14S-W-22569 & $4 / 4 / 07$ & 114.60 & $0.9 \mathrm{~J}$ & ND & ND \\
\hline & & BAMW14S-W-22613 & $11 / 18 / 07$ & 106.75 & 1.2 & ND & ND \\
\hline & & BAMW14S-W-22640 & $3 / 8 / 08$ & 106.95 & 4.3 & $0.3 \mathrm{~J}$ & ND \\
\hline & & BAMW14S-W-22689 & $7 / 10 / 08$ & 99.40 & 5.6 & $0.3 \mathrm{~J}$ & ND \\
\hline & & BAMW14S-W-27740 & $10 / 22 / 08$ & 96.20 & 5.6 & $0.3 \mathrm{~J}$ & ND \\
\hline & & BAMW14S-W-28620 & $3 / 4 / 09$ & 101.30 & 5.6 & $0.4 \mathrm{~J}$ & ND \\
\hline & & BAMW14S-W-28659 & 6/18/09 & 99.80 & 3.7 & $0.6 \mathrm{~J}$ & ND \\
\hline & & BAMW14S-W-28698 & 10/1/09 & 101.43 & 5.2 & $0.3 \mathrm{~J}$ & ND \\
\hline & & BAMW14S-W-28738 & $4 / 1 / 10$ & 96.70 & 4.3 & $0.3 \mathrm{~J}$ & ND \\
\hline & & BAMW14S-W-28781 & $9 / 18 / 10$ & 87.82 & 4.9 & $0.4 \mathrm{~J}$ & ND \\
\hline & & BAMW14S-W-28816 & $9 / 28 / 11$ & 101.40 & 3.4 & ND & ND \\
\hline \multirow[t]{10}{*}{ MW14D } & $123-133$ & BAMW14D-W-22568 & $4 / 4 / 07$ & 114.00 & 1.2 & ND & ND \\
\hline & & BAMW14D-W-22614 & $11 / 18 / 07$ & 107.10 & $0.6 \mathrm{~J}$ & ND & ND \\
\hline & & BAMW14D-W-22638 & $3 / 8 / 08$ & 106.95 & $0.7 \mathrm{~J}$ & ND & ND \\
\hline & & BAMW14D-W-22690 & $7 / 10 / 08$ & 101.00 & $0.5 \mathrm{~J}$ & ND & ND \\
\hline & & BAMW14D-W-27741 & $10 / 22 / 08$ & 96.10 & ND & ND & ND \\
\hline & & BAMW14D-W-28621 & $3 / 5 / 09$ & 103.20 & $0.6 \mathrm{~J}$ & ND & ND \\
\hline & & BAMW14D-W-28660 & $6 / 18 / 09$ & 97.75 & ND & ND & ND \\
\hline & & BAMW14D-W-28699 & 10/1/09 & 101.48 & $0.5 \mathrm{~J}$ & ND & ND \\
\hline & & BAMW14D-W-28739 & $4 / 1 / 10$ & 96.50 & $0.4 \mathrm{~J}$ & ND & ND \\
\hline & & BAMW14D-W-28782 & $9 / 17 / 10$ & 87.66 & ND & ND & ND \\
\hline \multirow[t]{10}{*}{ MW15S } & $88-98$ & BAMW15S-W-22560 & $4 / 4 / 07$ & 91.50 & 1.5 & ND & ND \\
\hline & & BAMW15S-W-22615 & $11 / 18 / 07$ & 84.33 & 8.7 & $0.4 \mathrm{~J}$ & ND \\
\hline & & BAMW15S-W-22648 & $3 / 10 / 08$ & 84.66 & 1.8 & $0.2 \mathrm{~J}$ & ND \\
\hline & & BAMW15S-W-22691 & $7 / 12 / 08$ & 80.30 & 2.2 & $0.3 \mathrm{~J}$ & ND \\
\hline & & BAMW15S-W-27742 & $10 / 23 / 08$ & 73.20 & 1.9 & ND & ND \\
\hline & & BAMW15S-W-28622 & $3 / 5 / 09$ & 73.80 & 2.5 & ND & ND \\
\hline & & BAMW15S-W-28661 & $6 / 17 / 09$ & 75.92 & 3.2 & $0.5 \mathrm{~J}$ & ND \\
\hline & & BAMW15S-W-28700 & $9 / 29 / 09$ & 79.45 & 2.6 & ND & ND \\
\hline & & BAMW15S-W-28740 & $3 / 30 / 10$ & 75.65 & 4.0 & $0.4 \mathrm{~J}$ & ND \\
\hline & & BAMW15S-W-28783 & $9 / 18 / 10$ & 66.07 & 1.9 & ND & ND \\
\hline
\end{tabular}


TABLE 4.3 (Cont.)

\begin{tabular}{|c|c|c|c|c|c|c|c|}
\hline \multirow[b]{2}{*}{ Location } & \multirow[b]{2}{*}{$\begin{array}{l}\text { Screen Interval } \\
\text { Depth } \\
\text { (ft BGL) }\end{array}$} & \multirow[b]{2}{*}{ Sample } & \multirow[b]{2}{*}{$\begin{array}{l}\text { Sample } \\
\text { Date }\end{array}$} & \multirow[b]{2}{*}{$\begin{array}{l}\text { Depth to } \\
\text { Water } \\
\text { (ft BTOC) }\end{array}$} & \multicolumn{3}{|c|}{ Concentration $(\mu \mathrm{g} / \mathrm{L})$} \\
\hline & & & & & $\begin{array}{c}\text { Carbon } \\
\text { Tetrachloride }\end{array}$ & Chloroform & $\begin{array}{l}\text { Methylene } \\
\text { Chloride }\end{array}$ \\
\hline
\end{tabular}

CCC/USDA wells installed during the 2006-2007 investigation (cont.)

MW15D 105-115

MW16S 76-86

MW16D 90-100

MW17 120-130

Private wells

Oentrich 150
BAMW15D-W-22561

BAMW15D-W-22616

BAMW15D-W-22631

BAMW15D-W-22692

BAMW15D-W-27743

BAMW15D-W-28623

BAMW15D-W-28662

BAMW15D-W-28701

BAMW15D-W-28741

BAMW15D-W-28784

BAMW16S-W-22563

BAMW16S-W-22617

BAMW16S-W-22632

BAMW16S-W-22693

BAMW16S-W-27744

BAMW16S-W-28624

BAMW16S-W-28663

BAMW16S-W-28702

BAMW16S-W-28742

BAMW16S-W-28785

BAMW16D-W-22562

BAMW16D-W-22618

BAMW16D-W-22633

BAMW16D-W-22694

BAMW16D-W-27745

BAMW16D-W-28625

BAMW16D-W-28664

BAMW16D-W-28703

BAMW16D-W-28743

BAMW16D-W-28786

BAMW17D-W-22566

BAMW17D-W-22619

BAMW17-W-22634

BAMW17-W-22695

BAMW17-W-27746

BAMW17-W-28626

BAMW17-W-28665

BAMW17-W-28704

BAMW17-W-28744

BAMW17-W-28787

BAMW17-W-28817

BAOENT-W-21693

BAOENT-W-21713

BAOENTRICH-W-22579

BAOENTRICH-W-22622

BAOENTRICH-W-22654

BAOENTRICH-W-22695

BAOENTRICH-W-27747

BAOENTRICH-W-28627

BAOENTRICH-W-28666

BAOENTRICH-W-28705

BAOENTRICH-W-28745

BAOENTRICH-W-28788
$4 / 4 / 07$

$11 / 18 / 07 \quad 70.20$

$3 / 8 / 08 \quad 80.80$

$7 / 12 / 08 \quad 70.30$

$10 / 24 / 08 \quad 67.60$

$3 / 5 / 09 \quad 73.60$

$6 / 17 / 09 \quad 67.74$

$9 / 29 / 09 \quad 72.10$

$3 / 30 / 10 \quad 66.50$

$9 / 18 / 10 \quad 58.11$

$4 / 4 / 07 \quad 81.00$

$11 / 19 / 07 \quad 75.30$

$3 / 7 / 08 \quad 75.50$

$7 / 11 / 08 \quad 67.35$

$10 / 23 / 08 \quad 64.80$

$3 / 5 / 09 \quad 69.60$

$6 / 18 / 09 \quad 66.93$

$9 / 29 / 09 \quad 70.35$

$3 / 30 / 10 \quad 66.10$

$9 / 18 / 10 \quad 57.24$

$4 / 4 / 07 \quad 79.71$

$11 / 19 / 07$

$3 / 7 / 08 \quad 75.00$

$7 / 11 / 08 \quad 66.30$

$10 / 23 / 08 \quad 63.90$

$3 / 5 / 09 \quad 69.00$

$6 / 18 / 09 \quad 66.49$

$9 / 29 / 09 \quad 70.00$

$3 / 30 / 10 \quad 65.95$

$9 / 18 / 10 \quad 57.65$

$4 / 4 / 07 \quad 110.68$

$11 / 19 / 07 \quad 102.68$

$3 / 5 / 08 \quad 101.75$

$7 / 9 / 08 \quad 96.60$

$10 / 22 / 08 \quad 95.15$

$3 / 4 / 09 \quad 98.10$

$6 / 17 / 09 \quad 95.75$

$9 / 30 / 09 \quad 98.00$

$3 / 31 / 10 \quad 94.90$

$9 / 17 / 10 \quad 88.03$

$9 / 28 / 11 \quad 96.23$

$\begin{array}{ccc}\text { ND } & \text { ND } & \text { ND } \\ \text { ND } & \text { ND } & \text { ND } \\ 0.2 \mathrm{~J} & \text { ND } & \text { ND } \\ \text { ND } & \text { ND } & \text { ND } \\ \text { ND } & \text { ND } & \text { ND } \\ \text { ND } & \text { ND } & \text { ND } \\ \text { ND } & \text { ND } & \text { ND } \\ \text { ND } & \text { ND } & \text { ND } \\ \text { ND } & \text { ND } & \text { ND } \\ \text { ND } & \text { ND } & \text { ND } \\ & & \\ \text { ND } & \text { ND } & \text { ND } \\ \text { ND } & \text { ND } & \text { ND } \\ 0.4 \mathrm{~J} & \text { ND } & \text { ND } \\ \text { ND } & \text { ND } & \text { ND } \\ 0.9 \mathrm{~J} & \text { ND } & \text { ND } \\ 1.4 & \text { ND } & \text { ND } \\ 1.6 & \text { ND } & \text { ND } \\ 1.7 & \text { ND } & \text { ND } \\ 1.6 & \text { ND } & \text { ND } \\ 1.7 & \text { ND } & \text { ND }\end{array}$

ND

ND

ND

ND

ND

ND

ND

ND

ND

ND

ND

ND

$0.3 \mathrm{~J}$

$0.4 \mathrm{~J}$

$0.7 \mathrm{~J}$

1.0

1.0

ND

$0.5 \mathrm{~J}$

ND

$0.4 \mathrm{~J}$

$\begin{array}{ll}\text { ND } & \text { ND } \\ \text { ND } & \text { ND } \\ \text { ND } & \text { ND } \\ \text { ND } & \text { ND } \\ \text { ND } & \text { ND } \\ \text { ND } & \text { ND } \\ \text { ND } & \text { ND } \\ \text { ND } & \text { ND } \\ \text { ND } & \text { ND } \\ \text { ND } & \text { ND }\end{array}$

ND ND

ND ND

ND ND

ND ND

ND ND

ND ND

ND ND

ND ND

ND ND

ND ND

ND ND

\begin{tabular}{|c|c|c|c|c|}
\hline $7 / 20 / 06$ & - & $0.3 \mathrm{~J}$ & ND & ND \\
\hline $8 / 2 / 06$ & - & $0.6 \mathrm{~J}$ & ND & ND \\
\hline $4 / 5 / 07$ & - & $0.6 \mathrm{~J}$ & ND & ND \\
\hline $11 / 19 / 07$ & - & $0.8 \mathrm{~J}$ & ND & ND \\
\hline $3 / 6 / 08$ & - & 1.3 & ND & ND \\
\hline $7 / 11 / 08$ & - & $0.3 \mathrm{~J}$ & ND & ND \\
\hline $10 / 23 / 08$ & - & $0.9 \mathrm{~J}$ & ND & ND \\
\hline $3 / 5 / 09$ & - & 1.1 & ND & ND \\
\hline 6/18/09 & - & $0.9 \mathrm{~J}$ & ND & ND \\
\hline 9/30/09 & - & 1.6 & ND & ND \\
\hline $4 / 1 / 10$ & - & 1.2 & ND & ND \\
\hline $9 / 18 / 10$ & - & 3.3 & $0.8 \mathrm{~J}$ & ND \\
\hline
\end{tabular}


TABLE 4.3 (Cont.)

\begin{tabular}{|c|c|c|c|c|c|c|c|}
\hline \multirow[b]{2}{*}{ Location } & \multirow[b]{2}{*}{$\begin{array}{l}\text { Screen Interval } \\
\text { Depth } \\
\text { (ft BGL) }\end{array}$} & \multirow[b]{2}{*}{ Sample } & \multirow[b]{2}{*}{$\begin{array}{l}\text { Sample } \\
\text { Date }\end{array}$} & \multirow[b]{2}{*}{$\begin{array}{l}\text { Depth to } \\
\text { Water } \\
\text { (ft BTOC) })^{\mathrm{a}}\end{array}$} & \multicolumn{3}{|c|}{ Concentration $(\mu \mathrm{g} / \mathrm{L})$} \\
\hline & & & & & $\begin{array}{c}\text { Carbon } \\
\text { Tetrachloride }\end{array}$ & Chloroform & $\begin{array}{l}\text { Methylene } \\
\text { Chloride }\end{array}$ \\
\hline \multicolumn{8}{|c|}{ Private wells (cont.) } \\
\hline Sedivy & 138 & $\begin{array}{l}\text { BACW-W-21849 } \\
\text { BASED2-W-21913 }\end{array}$ & $\begin{array}{l}8 / 22 / 06 \\
9 / 13 / 06\end{array}$ & $\begin{array}{l}- \\
-\end{array}$ & $\begin{array}{l}\text { ND } \\
\text { ND }\end{array}$ & $\begin{array}{l}\text { ND } \\
\text { ND }\end{array}$ & $\begin{array}{l}\text { ND } \\
\text { ND }\end{array}$ \\
\hline Sedivy1 & 90 & Not sampled (well dry) & $9 / 13 / 06$ & - & - & - & - \\
\hline \multicolumn{8}{|c|}{ Public water supply wells ${ }^{d}$} \\
\hline PWS2 & 155 & $\begin{array}{l}\text { BAPWS2-W-22510 } \\
\text { BAPW2-W-22578 } \\
\text { BAPW2-W-22620 } \\
\text { BAPWS2-W-22655 } \\
\text { BAPWS2-W-22696 } \\
\text { BAPW2-W-27748 } \\
\text { BAPWS2-W-28628 } \\
\text { BAPWS2-W-28667 } \\
\text { BAPWS2-W-28706 } \\
\text { BAPWS2-W-28715 } \\
\text { BAPWS2-W-28746 } \\
\text { BAPWS2-W-28758 } \\
\text { BAPWS2-W-28789 } \\
\text { BAPWS2-W-28803 } \\
\text { BAPWS2-W-28818 } \\
\text { BAPWS2-W-28827 }\end{array}$ & $\begin{array}{r}3 / 9 / 07 \\
4 / 5 / 07 \\
11 / 20 / 07 \\
3 / 6 / 08 \\
7 / 11 / 08 \\
10 / 23 / 08 \\
3 / 5 / 09 \\
6 / 18 / 09 \\
9 / 30 / 09 \\
12 / 14 / 09 \\
3 / 31 / 10 \\
6 / 17 / 10 \\
9 / 18 / 10 \\
12 / 15 / 10 \\
9 / 28 / 11 \\
12 / 12 / 11\end{array}$ & $\begin{array}{l}- \\
- \\
- \\
- \\
- \\
- \\
- \\
- \\
- \\
- \\
- \\
- \\
- \\
- \\
- \\
-\end{array}$ & $\begin{array}{l}\text { ND } \\
\text { ND } \\
\text { ND } \\
\text { ND } \\
0.8 \mathrm{~J} \\
1.7 \\
0.9 \mathrm{~J} \\
1.0 \\
\text { ND } \\
\text { ND } \\
0.9 \mathrm{~J} \\
0.8 \mathrm{~J} \\
1.1 \\
0.7 \mathrm{~J} \\
0.6 \mathrm{~J} \\
N D\end{array}$ & $\begin{array}{l}\text { ND } \\
\text { ND } \\
\text { ND } \\
\text { ND } \\
\text { ND } \\
\text { ND } \\
\text { ND } \\
\text { ND } \\
\text { ND } \\
\text { ND } \\
\text { ND } \\
\text { ND } \\
\text { ND } \\
\text { ND } \\
\text { ND } \\
\text { ND }\end{array}$ & $\begin{array}{l}\text { ND } \\
\text { ND } \\
\text { ND } \\
\text { ND } \\
\text { ND } \\
\text { ND } \\
\text { ND } \\
\text { ND } \\
\text { ND } \\
\text { ND } \\
\text { ND } \\
\text { ND } \\
\text { ND } \\
\text { ND } \\
\text { ND } \\
\text { ND }\end{array}$ \\
\hline PWS3 & 160 & $\begin{array}{l}\text { BAPWS3-W-22511 } \\
\text { BAPW3-W-22577 } \\
\text { BAPW3-W-22621 } \\
\text { BAPWS3-W-22656 } \\
\text { BAPWS3-W-22697 } \\
\text { BAPW3-W-27749 } \\
\text { BAPWS3-W-28629 } \\
\text { BAPWS3-W-28668 } \\
\text { BAPWS3-W-28707 } \\
\text { BAPWS3-W-28716 } \\
\text { BAPWS3-W-28747 } \\
\text { BAPWS3-W-28759 } \\
\text { BAPWS3-W-28790 } \\
\text { BAPWS3-W-28804 } \\
\text { BAPWS3-W-28819 } \\
\text { BAPWS3-W-28828 }\end{array}$ & $\begin{array}{r}3 / 9 / 07 \\
4 / 5 / 07 \\
11 / 20 / 07 \\
3 / 6 / 08 \\
7 / 11 / 08 \\
10 / 23 / 08 \\
3 / 5 / 09 \\
6 / 18 / 09 \\
9 / 30 / 09 \\
12 / 14 / 09 \\
3 / 31 / 10 \\
6 / 17 / 10 \\
9 / 18 / 10 \\
12 / 15 / 10 \\
9 / 28 / 11 \\
12 / 12 / 11\end{array}$ & $\begin{array}{l}- \\
- \\
- \\
- \\
- \\
- \\
- \\
- \\
- \\
- \\
- \\
- \\
- \\
- \\
- \\
-\end{array}$ & $\begin{array}{l}0.2 \mathrm{~J} \\
\text { ND } \\
\text { ND } \\
\text { ND } \\
0.2 \mathrm{~J} \\
\text { ND } \\
\text { ND } \\
\text { ND } \\
\text { ND } \\
\text { ND } \\
\text { ND } \\
\text { ND } \\
\text { ND } \\
\text { ND } \\
\text { ND } \\
\text { ND }\end{array}$ & $\begin{array}{l}\text { ND } \\
\text { ND } \\
\text { ND } \\
\text { ND } \\
\text { ND } \\
\text { ND } \\
\text { ND } \\
\text { ND } \\
\text { ND } \\
\text { ND } \\
\text { ND } \\
\text { ND } \\
\text { ND } \\
\text { ND } \\
2.2 \\
\text { ND }\end{array}$ & $\begin{array}{l}\text { ND } \\
\text { ND } \\
\text { ND } \\
\text { ND } \\
\text { ND } \\
\text { ND } \\
\text { ND } \\
\text { ND } \\
\text { ND } \\
\text { ND } \\
\text { ND } \\
\text { ND } \\
\text { ND } \\
\text { ND } \\
\text { ND } \\
\text { ND }\end{array}$ \\
\hline
\end{tabular}


TABLE 4.3 (Cont.)

\begin{tabular}{|c|c|c|c|c|c|c|c|}
\hline \multirow[b]{2}{*}{ Location } & \multirow[b]{2}{*}{$\begin{array}{c}\text { Screen Interval } \\
\text { Depth } \\
\text { (ft BGL) }\end{array}$} & \multirow[b]{2}{*}{ Sample } & \multirow[b]{2}{*}{$\begin{array}{l}\text { Sample } \\
\text { Date }\end{array}$} & \multirow[b]{2}{*}{$\begin{array}{l}\text { Depth to } \\
\text { Water } \\
(\mathrm{ft} \mathrm{BTOC})^{\mathrm{a}}\end{array}$} & \multicolumn{3}{|c|}{ Concentration $(\mu \mathrm{g} / \mathrm{L})$} \\
\hline & & & & & $\begin{array}{c}\text { Carbon } \\
\text { Tetrachloride }\end{array}$ & Chloroform & $\begin{array}{l}\text { Methylene } \\
\text { Chloride }\end{array}$ \\
\hline
\end{tabular}

a BTOC, below top of casing.

b ND, contaminant not detected at an instrument detection limit of $0.1 \mu \mathrm{g} / \mathrm{L}$.

c Qualifier J indicates an estimated concentration below the purge-and-trap method quantitation limit of $1.0 \mu \mathrm{g} / \mathrm{L}$.

d Pumping status of public wells:

\begin{tabular}{|c|c|}
\hline $\begin{array}{l}\text { Sampling } \\
\text { Date }\end{array}$ & PWS2 \\
\hline 3/9/07 & Well has been pumping today. \\
\hline $4 / 5 / 07$ & Sampled after letting run for 5-10 min. \\
\hline $11 / 20 / 07$ & Well on at time of sampling. \\
\hline 3/6/08 & Let water run from tap for $2-3$ min, then sampled. \\
\hline $7 / 11 / 08$ & Running for $30 \mathrm{~min}$. \\
\hline 10/23/08 & Well was pumping for $5 \mathrm{~min}$. \\
\hline $3 / 5 / 09$ & Well operating to fill water tower prior to sampling. \\
\hline $6 / 18 / 09$ & $\begin{array}{l}\text { Well pumping since } 6 \text { a.m. on June 18. Let run } \\
\text { from tap for } 5 \text { min, then sampled. }\end{array}$ \\
\hline 9/30/09 & $\begin{array}{l}\text { Well used on September 29. Let well run for } 10 \\
\text { min, then sampled. }\end{array}$ \\
\hline $12 / 14 / 09$ & Well has been pumping today. \\
\hline 3/31/10 & $\begin{array}{l}\text { Well used on March } 30 \text {. Let well run for } 10 \mathrm{~min} \text {, } \\
\text { then sampled. }\end{array}$ \\
\hline $6 / 17 / 10$ & Recent use of well not recorded in log. \\
\hline 9/18/10 & $\begin{array}{l}\text { Well in use for past } 2 \text { days. Sampled from tap after } \\
\text { purging for } 5-10 \text { min (approximately } 500 \text { gal). }\end{array}$ \\
\hline 12/15/10 & Recent use of well not recorded in log. \\
\hline 9/28/11 & Sampled from tap after purging for $5 \mathrm{~min}$. \\
\hline 12/12/11 & Sampled from tap after purging for $5 \mathrm{~min}$. \\
\hline
\end{tabular}

PWS3

Well has been pumping today.

Well has been pumping all day.

Well on at time of sampling.

Sample collected from tap in well house. Let water run from tap for 2-3 min, then sampled.

Running for $30 \mathrm{~min}$.

Well was pumping for $30 \mathrm{~min}$.

Ran for 5 min.

Well was used on June 17. Let pump run for 10 min, then sampled.

Well in use. Let tap run for $5 \mathrm{~min}$, then sampled.

Well has been pumping today.

Well in use oversnight. Let tap run for $5 \mathrm{~min}$, then sampled.

Recent use of well not recorded in log.

Well in use for past 2 days. Sampled from tap after purging for 5-10 min (approximately $500 \mathrm{gal}$ ).

Recent use of well not recorded in log.

Sampled from tap after purging for $5 \mathrm{~min}$.

Sampled from tap after purging for $5 \mathrm{~min}$. 


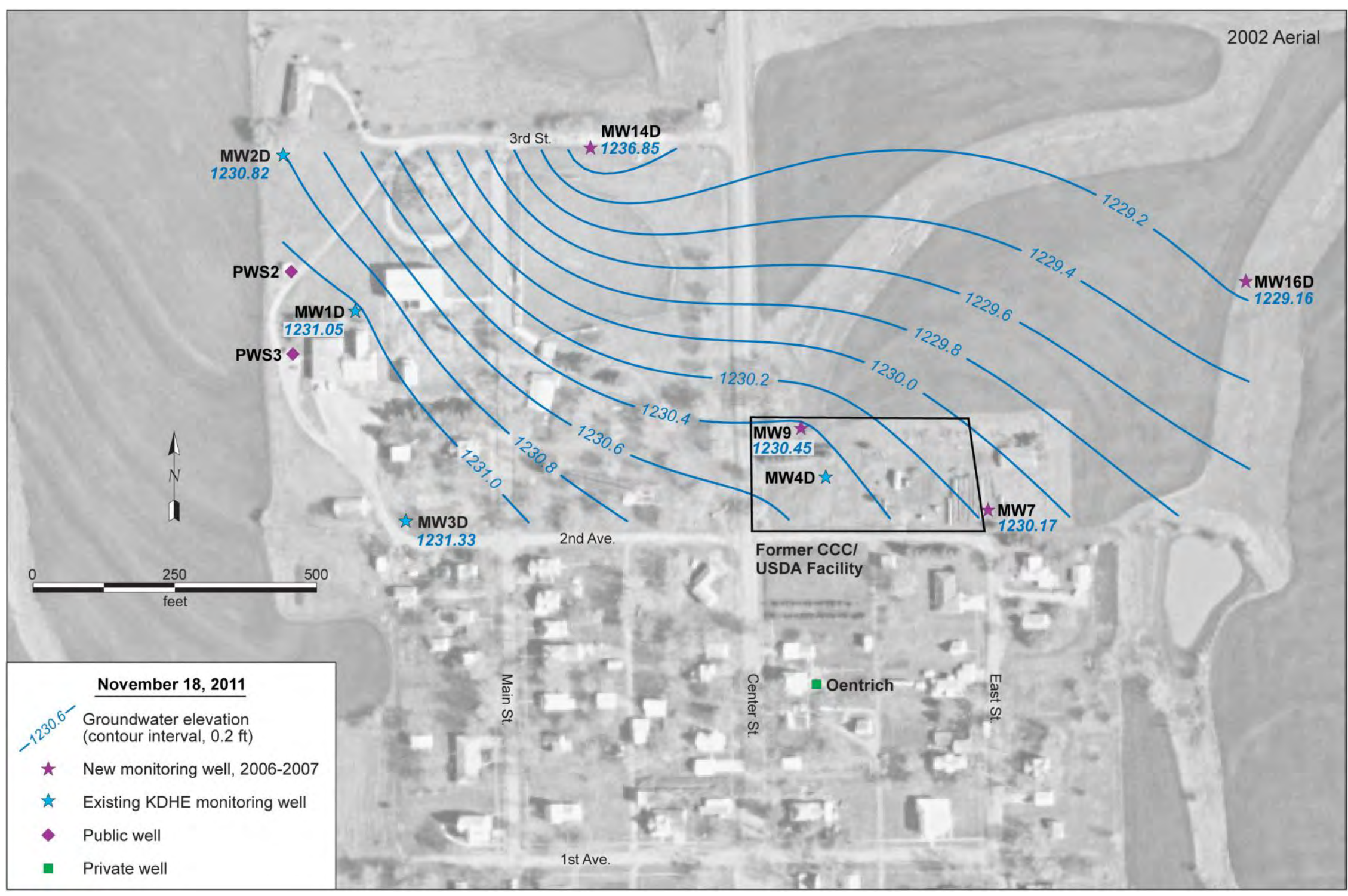

FIGURE 4.1 Potentiometric surface map depicting the groundwater flow direction in the deep aquifer zone under static (non-pumping) conditions on November 18, 2011. Source of photograph: NAPP (2002). 


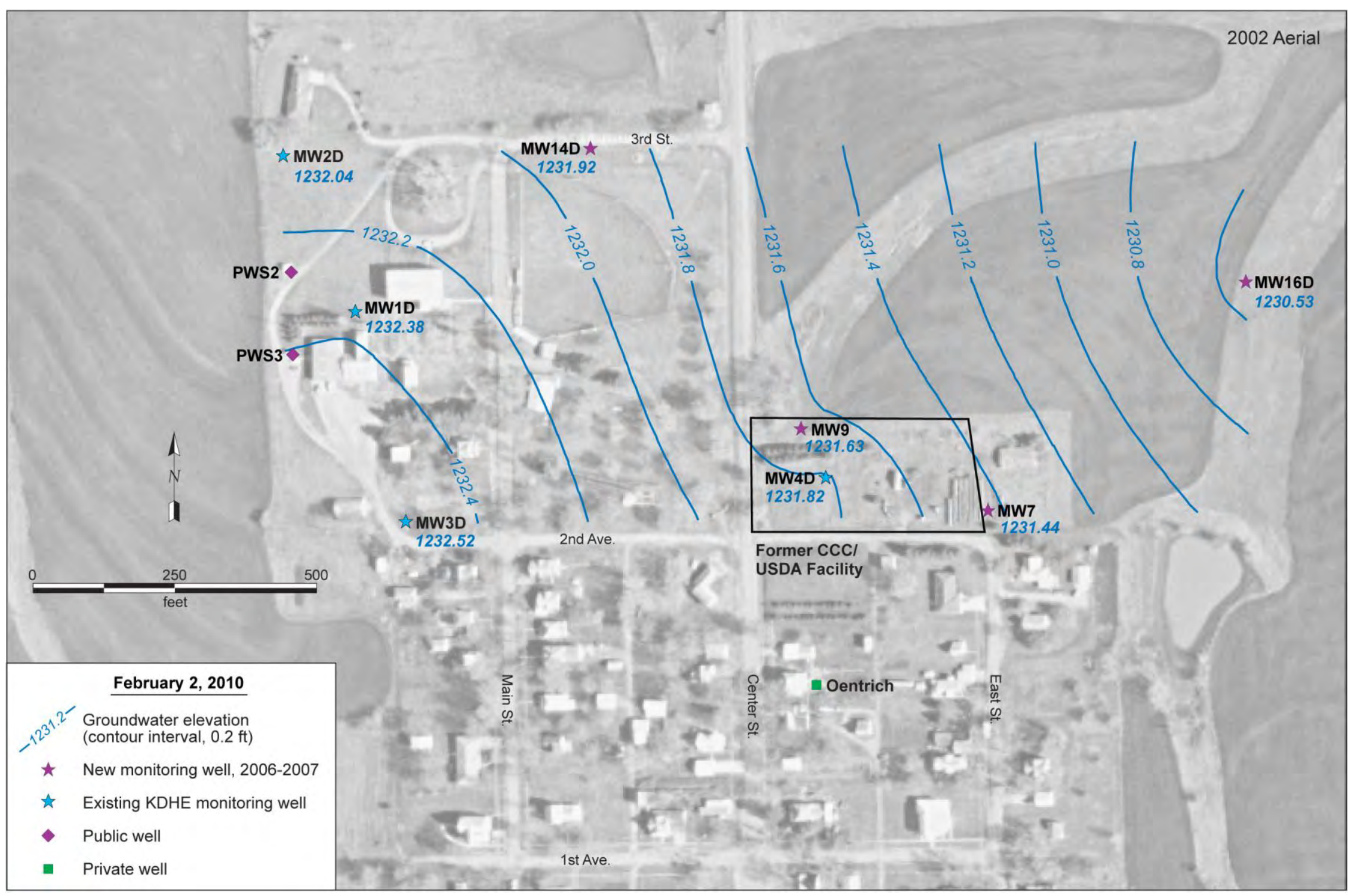

FIGURE 4.2a Potentiometric surface map depicting the groundwater flow direction in the deep aquifer zone under static (non-pumping) conditions on February 2, 2010. Source of photograph: NAPP (2002). 


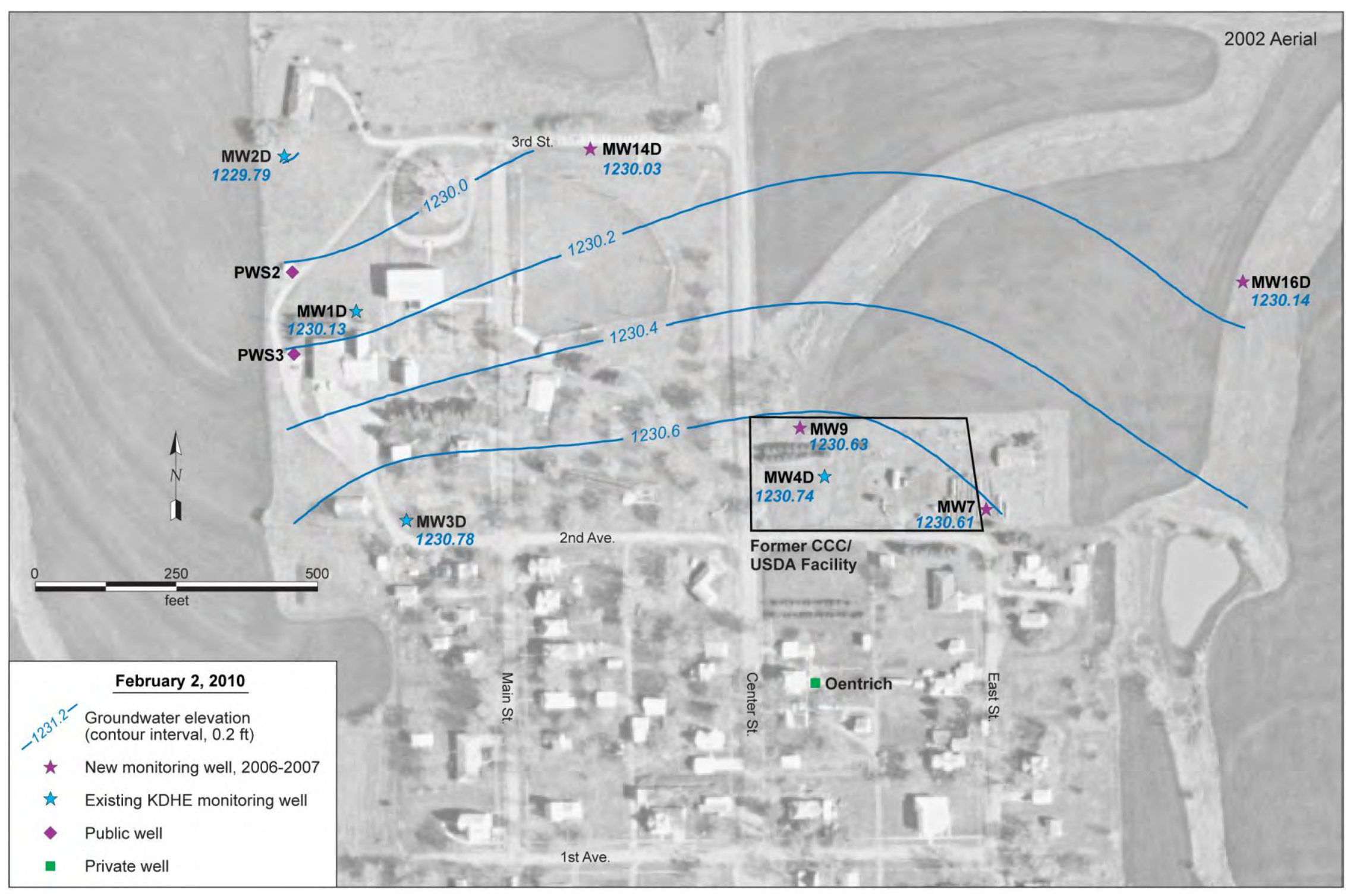

FIGURE 4.2b Potentiometric surface map depicting the groundwater flow direction in the deep aquifer zone under pumping conditions on February 2, 2010. Source of photograph: NAPP (2002). 


\section{Barnes Monitoring Data}

$1 / 1-6 / 30 / 11$

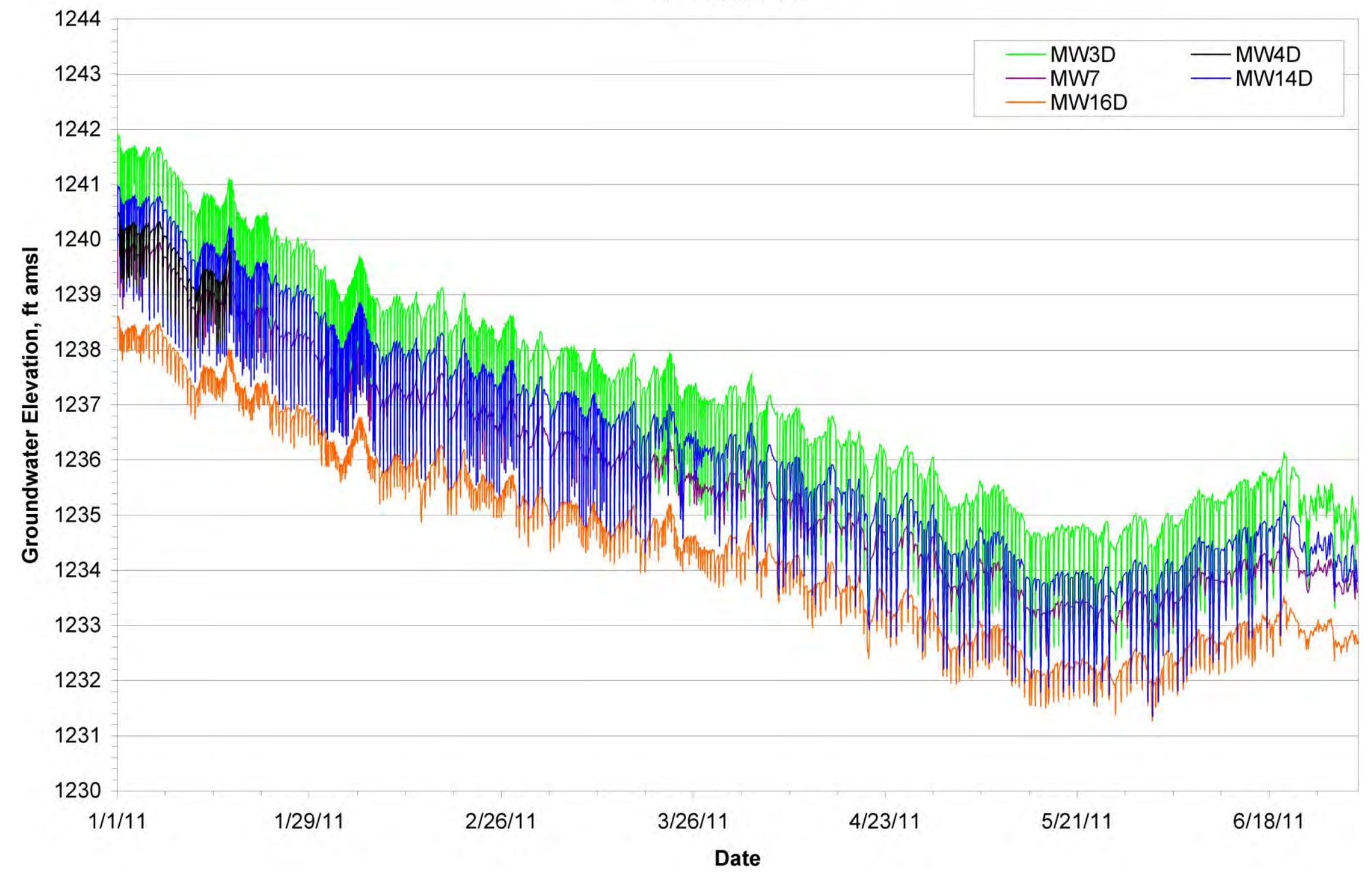

FIGURE 4.3a Hydrographs summarizing results of long-term water level monitoring in the deep-zone wells, January-June 2011. 


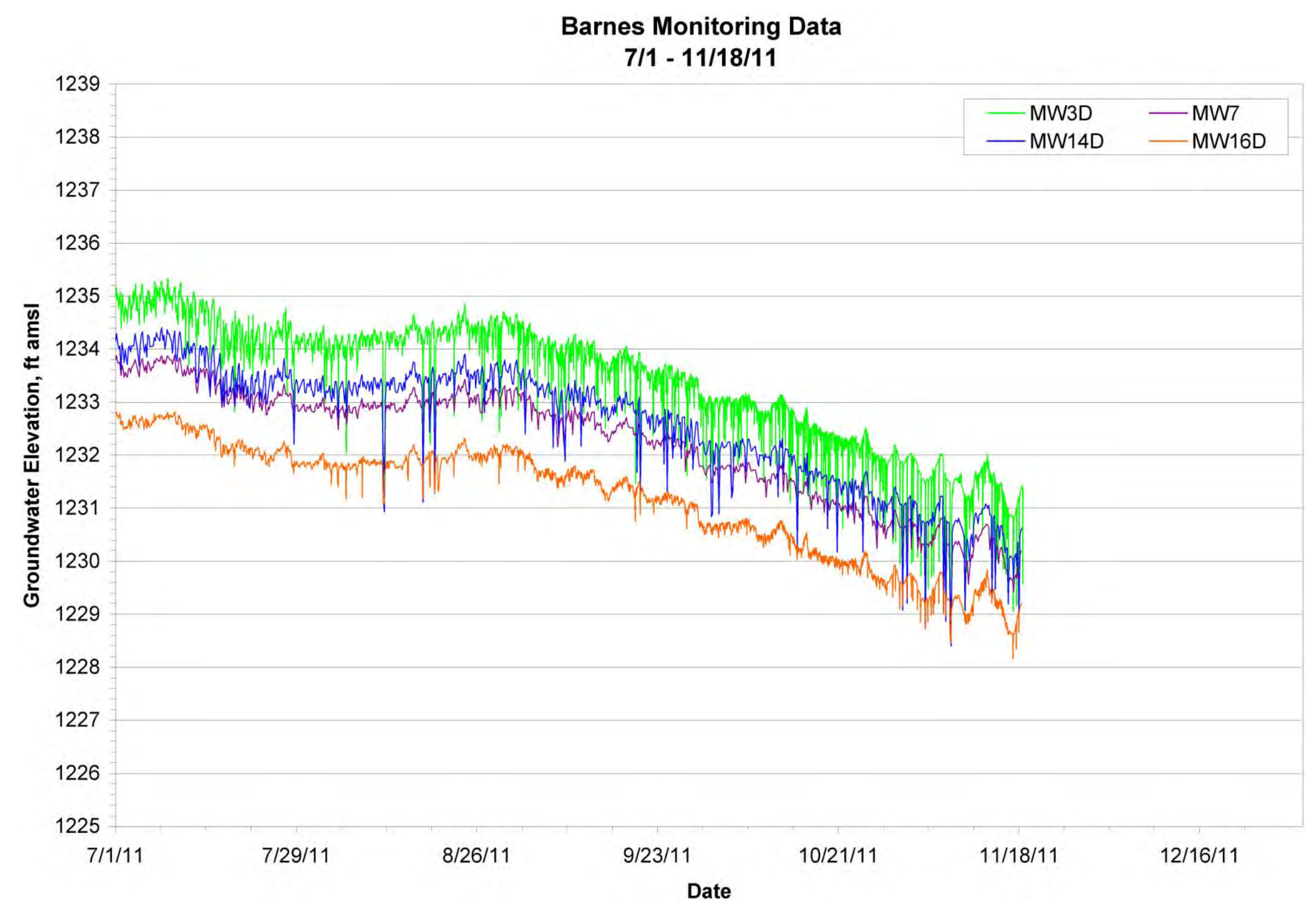

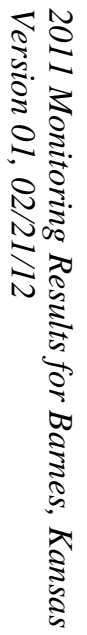

FIGURE 4.3b Hydrographs summarizing results of long-term water level monitoring in the deep-zone wells, July-November 2011. 


\section{Barnes Monitoring Data}

$6 / 1-6 / 30 / 11$

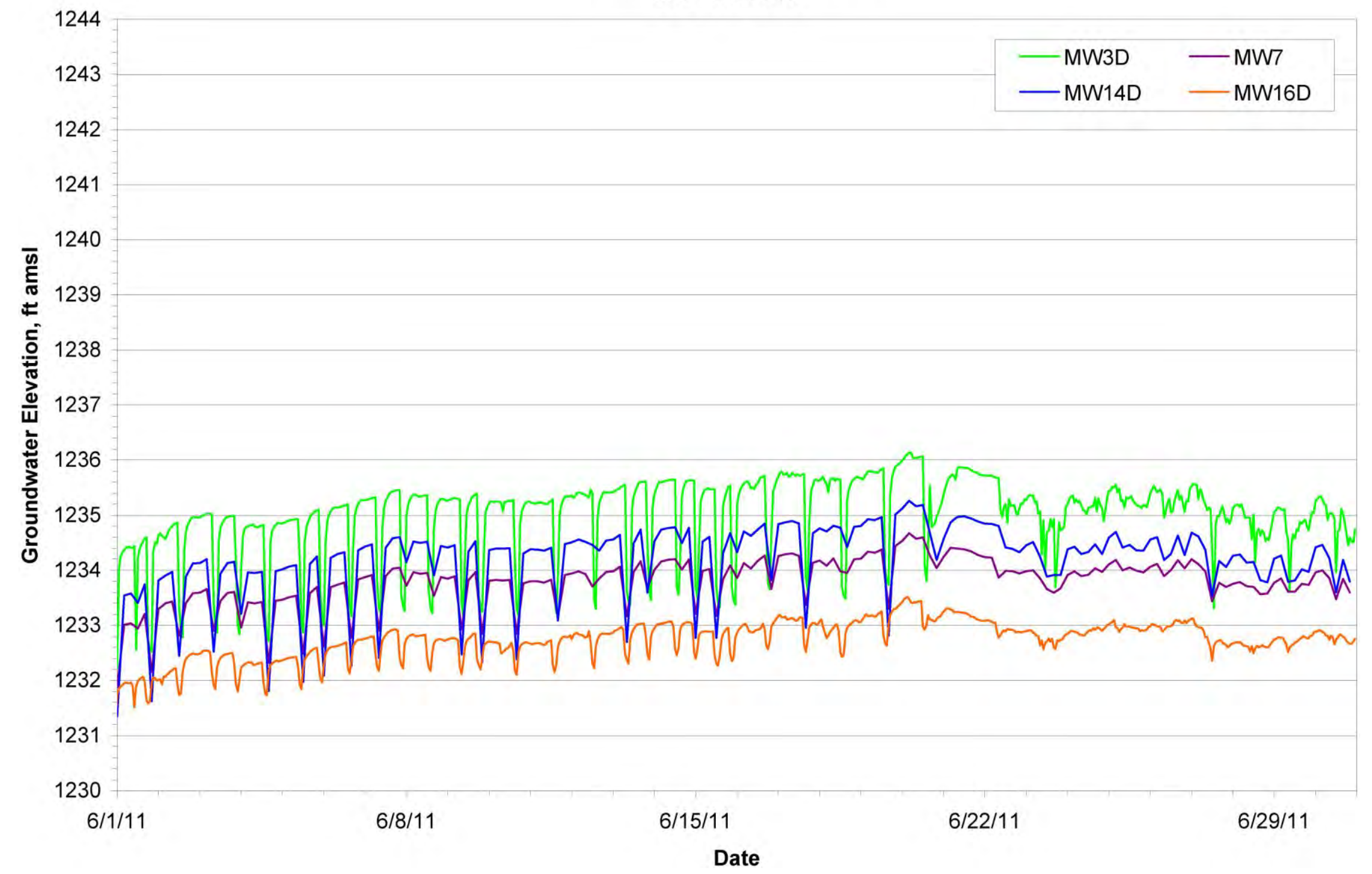

FIGURE 4.4 Hydrographs summarizing results of water level monitoring in the deep-zone wells, June 2011. 


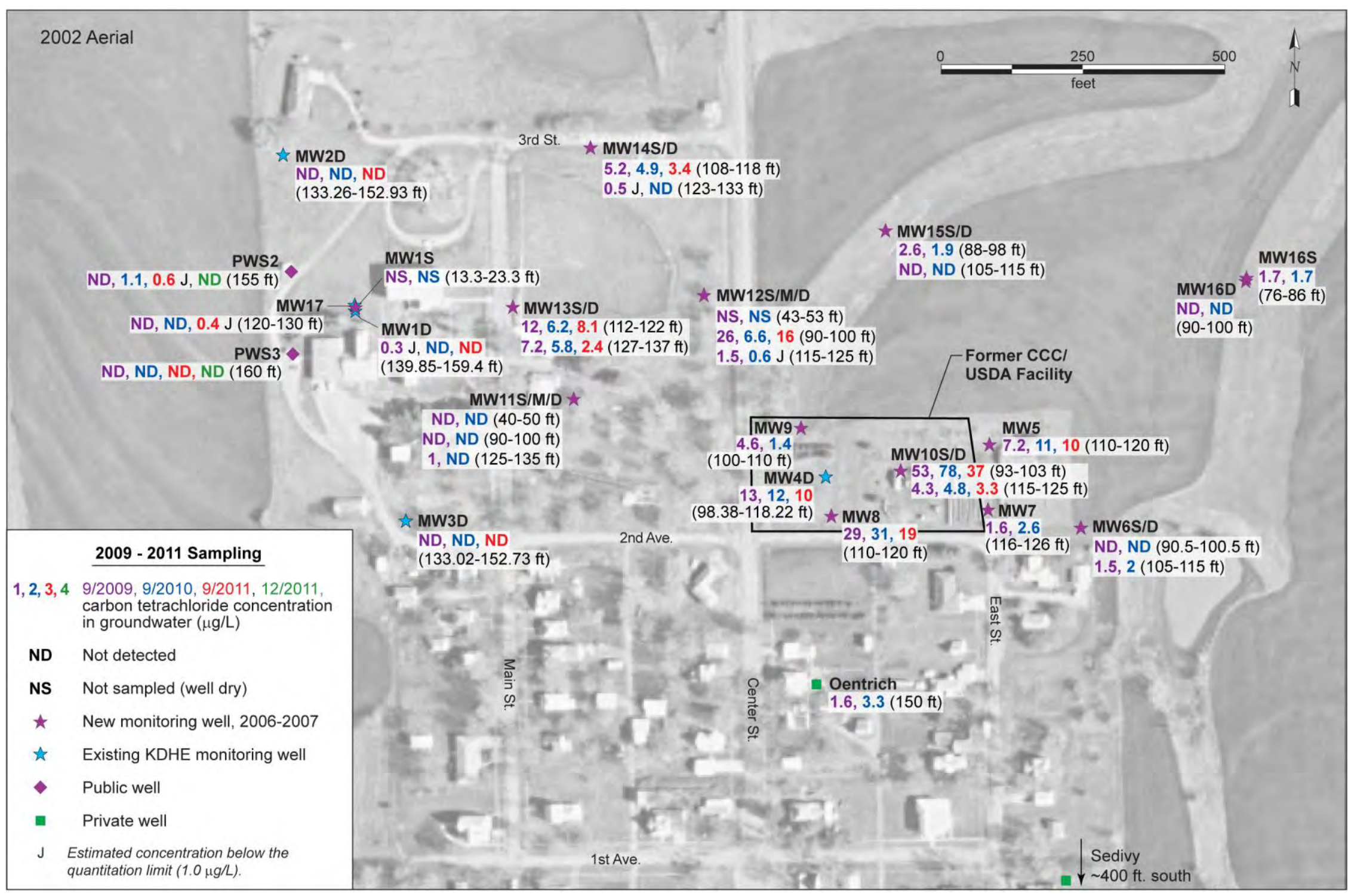

FIGURE 4.5 Analytical results for carbon tetrachloride in groundwater samples collected in 2009-2011. Source of photograph: NAPP (2002). 


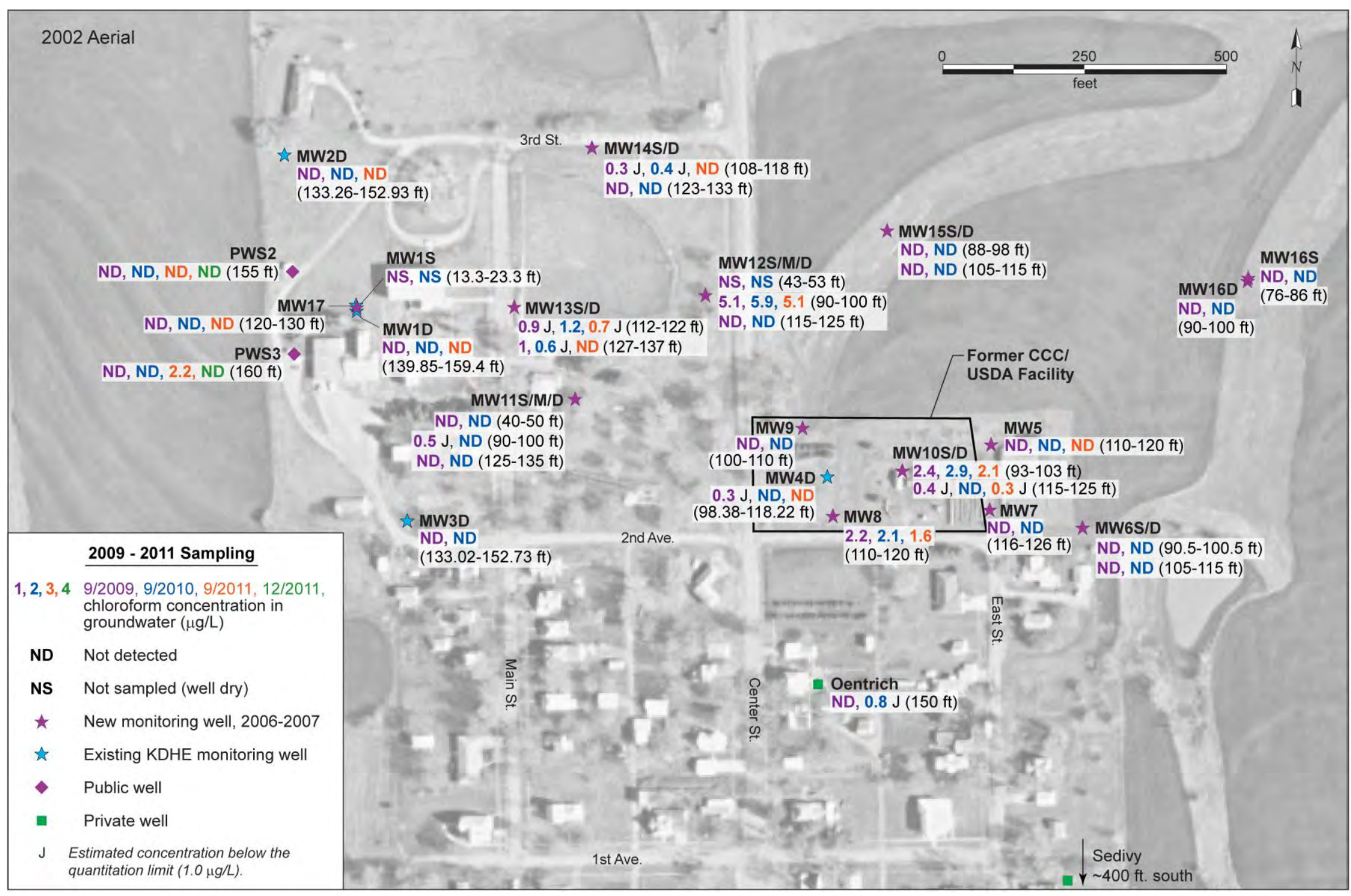

FIGURE 4.6 Analytical results for chloroform in groundwater samples collected in 2009-2011. Source of photograph: NAPP (2002). 


\section{Conclusions, Observations, and Recommendations}

\subsection{Conclusions}

The findings of the monitoring events at Barnes in 2011 continued to support the following previous conclusions:

- Measurements of groundwater levels obtained manually and through the use of automatic recorders have consistently indicated that the flow direction is strongly influenced by pumping of the public water supply wells. The results have demonstrated

- An apparent groundwater flow direction to the northeast when the public wells are not pumping and

- A northwesterly groundwater flow trend when the public wells are pumping.

- Evaluation of manual water level measurements and carbon tetrachloride concentrations continues to suggest that three vertically distinguishable aquifer zones are present at Barnes: shallow, intermediate, and deep (Table 4.1). The highest concentration of carbon tetrachloride occurs in the intermediate zone, in wells near the former CCC/USDA grain storage facility. Lower concentrations have been detected in the deep aquifer zone (where the public water supply wells are screened), and no carbon tetrachloride has been detected in the shallow zone.

- The conceptual model of the groundwater flow system at Barnes, as postulated on the basis of the accumulated results, suggests that the observed vertical hydraulic gradients and higher carbon tetrachloride concentrations in the intermediate zone might reflect generally lower permeability and hence less effective groundwater and contaminant migration in the intermediate zone than in the deep aquifer zone. 
- As it has since March 2008, intermediate-zone well MW10S, in the eastern portion of the former CCC/USDA facility, contained the highest concentrations of carbon tetrachloride.

- Overall, the lateral distribution of carbon tetrachloride in groundwater in 2011 is similar to the distribution during previous sampling events. The accumulated data, including a trend analysis conducted in 2009, indicate stable contaminant concentrations, with no imminent impact to the public wells.

\subsection{Observations in 2011}

The frequency and magnitude of the cyclic drawdown events historically observed in the hydrographs for the Barnes site changed in June 2011. Information from the city (Oentrich 2011) indicates that the changes are due to an upgrade of the municipal water delivery system during the summer. Whereas the municipal wells were previously switched on and off manually, sensors now control the pumping and maintain a more consistent water level in the city's water tower. The wells now operate more frequently than before, but for a much shorter duration during each pumping episode. Leaks in the former system were also repaired, thus reducing water usage.

These changes should minimize or eliminate the very appreciable drawdowns (up to $2.5 \mathrm{ft}$ during pumping) that previously caused transient reversal of the local groundwater flow pattern, from northeasterly under ambient conditions to northwesterly (toward the public wells) during pumping. The modified operating scheme might also moderate or prevent further movement of the contaminant plume across the site toward the northwest, ultimately reducing the risk of contamination to public water supply wells PWS2 and PWS3.

Continued groundwater sampling under the existing monitoring program will be required to determine the potential impacts of the operational changes on the distribution and concentrations of carbon tetrachloride at the site and the time frame over which such impacts might occur. In December 2011, no carbon tetrachloride was detected in the public wells. 


\subsection{Recommendations}

- Continue automatic water level measurements across the site investigation area (Figure 3.1).

- Continue annual sampling of the monitoring wells and semi-annual sampling of the public water supply wells (Figure 3.2).

- Through continued sampling and analysis, evaluate the potential impacts of operational changes in the public water distribution system. 


\section{References}

Argonne, 2002, Final Master Work Plan: Environmental Investigations at Former CCC/USDA Facilities in Kansas, 2002 Revision, ANL/ER/TR-02/004, prepared for the Commodity Credit Corporation, U.S. Department of Agriculture, Washington, D.C., by Argonne National Laboratory, Argonne, Illinois, December.

Argonne, 2006, Final Work Plan: Targeted Groundwater Sampling and Monitoring Well Installation for Potential Site Reclassification at Barnes, Kansas, ANL/EVS/AGEM/TR-06-04, prepared for the Commodity Credit Corporation, U.S. Department of Agriculture, by Argonne National Laboratory, Argonne, Illinois, May.

Argonne, 2008a, Final Report: Results of the 2006-2007 Investigation of Potential Contamination at the Former CCC/USDA Facility in Barnes, Kansas, ANL/EVS/AGEM/TR-0712, prepared for the Commodity Credit Corporation, U.S. Department of Agriculture, Washington, D.C., by Argonne National Laboratory, Argonne, Illinois, August.

Argonne, 2008b, November 2007 Monitoring Results for Barnes, Kansas, ANL/EVS/AGEM/TR-08-04, prepared for the Commodity Credit Corporation, U.S. Department of Agriculture, Washington, D.C., by Argonne National Laboratory, Argonne, Illinois, February.

Argonne, 2008c, March 2008 Monitoring Results for Barnes, Kansas, ANL/EVS/AGEM/TR-0811, prepared for the Commodity Credit Corporation, U.S. Department of Agriculture, Washington, D.C., by Argonne National Laboratory, Argonne, Illinois, August.

Argonne, 2008d, July 2008 Monitoring Results for Barnes, Kansas, ANL/EVS/AGEM/TR-0817, prepared for the Commodity Credit Corporation, U.S. Department of Agriculture, Washington, D.C., by Argonne National Laboratory, Argonne, Illinois, September.

Argonne, 2009a, October 2008 Monitoring Results for Barnes, Kansas, ANL/EVS/AGEM/TR09-04, prepared for the Commodity Credit Corporation, U.S. Department of Agriculture, Washington, D.C., by Argonne National Laboratory, Argonne, Illinois, February. 
Argonne, 2009b, March-June 2009 Monitoring Results for Barnes, Kansas, ANL/EVS/AGEM/TR-09-09, prepared for the Commodity Credit Corporation, U.S. Department of Agriculture, Washington, D.C., by Argonne National Laboratory, Argonne, Illinois, February.

Argonne, 2009c, Contingency Interim Measure for the Public Water Supply at Barnes, Kansas, ANL/EVS/AGEM/TR-08-19, prepared for the Commodity Credit Corporation, U.S. Department of Agriculture, Washington, D.C., by Argonne National Laboratory, Argonne, Illinois, June.

Argonne, 2010, Semi-Annual Monitoring Report for Barnes, Kansas, for 2010, ANL/EVS/AGEM/TR-10-02, prepared for the Commodity Credit Corporation, U.S. Department of Agriculture, Washington, D.C., by Argonne National Laboratory, Argonne, Illinois, April.

Argonne, 2011, Annual Report of Monitoring at Barnes, Kansas, in 2010, ANL/EVS/AGEM/TR-11-04, prepared for the Commodity Credit Corporation, U.S. Department of Agriculture, Washington, D.C., by Argonne National Laboratory, Argonne, Illinois, May.

BE\&K, 1999, Public Water Supply Site Comprehensive Investigation Barnes, Kansas, prepared for the Kansas Department of Health and Environment, Topeka, Kansas, by BE\&K/Terranext, March.

BE\&K, 2000, March 2000 Quarterly Monitoring Report: Barnes Public Water Supply Site Barnes, Kansas, prepared for the Kansas Department of Health and Environment, Topeka, Kansas, by BE\&K/Terranext, May.

EPA, 1995, Method 524.2: Measurement of Purgeable Organic Compounds in Water by Capillary Column Gas Chromatography/Mass Spectrometry, Revision 4.1, edited by J.W. Munch, National Exposure Research Laboratory, Office of Research and Development, U.S. Environmental Protection Agency, Cincinnati, Ohio.

KDHE, 2009, letter from E. Finzer (Bureau of Environmental Remediation, Kansas Department of Health and Environment, Topeka, Kansas) to C. Roe (Commodity Credit Corporation, U.S. Department of Agriculture, Washington, D.C.), regarding Contingency Interim Measure for the Public Water Supply at Barnes, Kansas, August 24. 
KDHE, 2010a, letter from M. Townsend (Bureau of Environmental Remediation, Kansas Department of Health and Environment, Topeka, Kansas) to C. Roe (Commodity Credit Corporation, U.S. Department of Agriculture, Washington, D.C.), regarding selection of monitoring wells, August 23.

KDHE, 2010b, letter from M. Townsend (Bureau of Environmental Remediation, Kansas Department of Health and Environment, Topeka, Kansas) to C. Roe (Commodity Credit Corporation, U.S. Department of Agriculture, Washington, D.C.), regarding request for annual monitoring, December 27.

NAPP, 2002, aerial image NAPP 12897 222, National Aerial Photography Program, U.S. Geological Survey (available from Kansas Geospatial Community Commons [http://www.kansasgis.org/]), April 9.

Oentrich, D., 2011, personal communication from Oentrich (City of Barnes, Kansas) to T. Kamler (TCW Construction, Lincoln, Nebraska), December 12.

PRC, 1996, Barnes Public Water Supply Site, Barnes, Washington County, Final Phase I Comprehensive Investigation Report, prepared for the Kansas Department of Health and Environment, Topeka, Kansas, by PRC Environmental Management, Inc., August.

Puls, R.W., and M.J. Barcelona, 1996, "Low-Flow (Minimal Drawdown) Ground-Water Sampling Procedures," EPA/540/S-95/504, in Ground Water Issue, Superfund Technology Support Center for Ground Water, National Risk Management Research Laboratory, U.S. Environmental Protection Agency, Ada, Oklahoma, April (www.epa.gov/tio/tsp/download/ lwflw2a.pdf).

USD 223, 1989, Inventory of Chemical Waste Accumulated, transmitted from D.L. Johnson (district superintendent) to J. Goetz (Hazardous Waste Section, Kansas Department of Health and Environment, Topeka, Kansas).

USGS, 1968, Barnes Quadrangle, Kansas, 7.5 Minute Series Topographical Map, photorevised 1983. 
Yeskis, D., and B. Zavala, 2002, Ground-Water Sampling Guidelines for Superfund and RCRA Project Managers: Ground Water Forum Issue Paper, EPA 542-S-02-001, Technology Innovative Office, Office of Solid Waste and Emergency Response, U.S. Environmental Protection Agency, Washington, D.C., May (http://www.epa.gov/tio/tsp/download/ gw_sampling_guide.pdf). 


\section{Appendix A:}

Sampling Activities and Field Measurements in 2011 
TABLE A.1 Sequence of groundwater sampling activities in 2011.

\begin{tabular}{|c|c|c|c|c|c|c|c|}
\hline $\begin{array}{c}\text { Sample } \\
\text { Date and Time }\end{array}$ & Sample & Type $^{a}$ & Location & $\begin{array}{l}\text { Depth } \\
\text { (ft BGL) }\end{array}$ & $\begin{array}{l}\text { Chain of } \\
\text { Custody }\end{array}$ & $\begin{array}{l}\text { Shipping } \\
\text { Date }\end{array}$ & Sample Description \\
\hline $9 / 28 / 1110: 52$ & BAMW13S-W-28814 & MW & MW13S & $112-122$ & 6631 & $9 / 28 / 11$ & $\begin{array}{l}\text { Depth to water }=87.55 \mathrm{ft} \text {. Depth of } 2 \text {-in. well }=122 \mathrm{ft} \text {. } \\
\text { Sample collected by using low-flow bladder pump } \\
\text { after purging of } 5 \mathrm{~L} \text {. Pump intake positioned at } 117 \mathrm{ft} \text {. }\end{array}$ \\
\hline 9/28/11 12:10 & BAMW2D-W-28807 & MW & MW2D & $133.26-152.93$ & 6631 & $9 / 28 / 11$ & $\begin{array}{l}\text { Depth to water }=116.22 \mathrm{ft} \text {. Depth of } 2 \text {-in. well }= \\
152.93 \mathrm{ft} \text {. Sample collected by using low-flow bladder } \\
\text { pump after purging of } 8.5 \mathrm{~L} \text {. Pump intake positioned } \\
\text { at } 143.10 \mathrm{ft} \text {. }\end{array}$ \\
\hline 9/28/11 12:52 & BAMW13D-W-28815 & MW & MW13D & $127-137$ & 6631 & $9 / 28 / 11$ & $\begin{array}{l}\text { Depth to water }=110.20 \mathrm{ft} \text {. Depth of } 2 \text {-in. well }=137 \mathrm{ft} \text {. } \\
\text { Sample collected by using low-flow bladder pump } \\
\text { after purging of } 5 \mathrm{~L} \text {. Pump intake positioned at } 132 \mathrm{ft} \text {. }\end{array}$ \\
\hline $9 / 28 / 1115: 10$ & BAMW17-W-28817 & MW & MW17 & $120-130$ & 6631 & $9 / 28 / 11$ & $\begin{array}{l}\text { Depth to water }=96.23 \mathrm{ft} \text {. Depth of } 2 \text {-in. well }=130 \mathrm{ft} \text {. } \\
\text { Sample collected by using low-flow bladder pump } \\
\text { after purging of } 4.5 \mathrm{~L} \text {. Pump intake positioned at } \\
125 \mathrm{ft} \text {. }\end{array}$ \\
\hline 9/28/11 15:36 & BAMW1D-W-28806 & MW & MW1D & $139.85-159.4$ & 6631 & $9 / 28 / 11$ & $\begin{array}{l}\text { Depth to water }=119.12 \mathrm{ft} \text {. Depth of } 2 \text {-in. well }= \\
159.4 \mathrm{ft} \text {. Sample collected by using low-flow bladder } \\
\text { pump after purging of } 7 \mathrm{~L} \text {. Pump intake positioned at } \\
149.63 \mathrm{ft} \text {. }\end{array}$ \\
\hline $9 / 28 / 1115: 37$ & BAMW1DDUP-W-28820 & MW & MW1D & $139.85-159.4$ & 6631 & $9 / 28 / 11$ & Replicate of sample BAMW1D-W-28806. \\
\hline $9 / 28 / 1116: 40$ & BAMW14S-W-28816 & MW & MW14S & $108-118$ & 6631 & $9 / 28 / 11$ & $\begin{array}{l}\text { Depth to water }=101.40 \mathrm{ft} \text {. Depth of } 2 \text {-in. well }=118 \mathrm{ft} \text {. } \\
\text { Sample collected by using low-flow bladder pump } \\
\text { after purging of } 5 \mathrm{~L} \text {. Pump intake positioned at } 113 \mathrm{ft} \text {. }\end{array}$ \\
\hline $9 / 28 / 1117: 19$ & BAMW12M-W-28813 & MW & MW12M & $90-100$ & 6631 & $9 / 28 / 11$ & $\begin{array}{l}\text { Depth to water }=71.25 \mathrm{ft} \text {. Depth of } 2 \text {-in. well }=100 \mathrm{ft} \text {. } \\
\text { Sample collected by using low-flow bladder pump } \\
\text { after purging of } 11 \mathrm{~L} \text {. Pump intake positioned at } 95 \mathrm{ft} \text {. }\end{array}$ \\
\hline $9 / 28 / 1117: 20$ & BAMW12MDUP-W-28821 & MW & MW12M & $90-100$ & 6631 & $9 / 28 / 11$ & Replicate of sample BAMW12M-W-28813. \\
\hline $9 / 28 / 1117: 45$ & BAQCIR-W-28822 & $\mathrm{RI}$ & QC & - & 6633 & $9 / 29 / 11$ & $\begin{array}{l}\text { Rinsate of decontaminated sampling line after collection } \\
\text { of sample BAMW12M-W-28813 and replicate } \\
\text { BAMW12MDUP-W-28821. }\end{array}$ \\
\hline $9 / 28 / 1117: 54$ & BAMW10D-W-28812 & MW & MW10D & $115-125$ & 6631 & $9 / 28 / 11$ & $\begin{array}{l}\text { Depth to water }=99.65 \mathrm{ft} \text {. Depth of } 2 \text {-in. well }=125 \mathrm{ft} \text {. } \\
\text { Sample collected by using low-flow bladder pump } \\
\text { after purging of } 7 \mathrm{~L} \text {. Pump intake positioned at } 120 \mathrm{ft} \text {. }\end{array}$ \\
\hline 9/28/11 18:03 & BAQCIR-W-28823b & $\mathrm{RI}$ & QC & - & 6633 & $9 / 29 / 11$ & $\begin{array}{l}\text { Rinsate of decontaminated sampling line after collection } \\
\text { of sample BAMW10D-W-28812. }\end{array}$ \\
\hline $9 / 28 / 11$ 18:34 & BADIH2O-W-28824b & $\mathrm{FB}$ & QC & - & 6633 & $9 / 29 / 11$ & $\begin{array}{l}\text { Field blank of water used for equipment } \\
\text { decontamination during September } 2011 \text { sampling } \\
\text { event. }\end{array}$ \\
\hline
\end{tabular}


TABLE A.1 (Cont.)

\begin{tabular}{|c|c|c|c|c|c|c|c|}
\hline $\begin{array}{c}\text { Sample } \\
\text { Date and Time }\end{array}$ & Sample & Type ${ }^{a}$ & Location & $\begin{array}{l}\text { Depth } \\
\text { (ft BGL) }\end{array}$ & $\begin{array}{l}\text { Chain of } \\
\text { Custody }\end{array}$ & $\begin{array}{l}\text { Shipping } \\
\text { Date }\end{array}$ & Sample Description \\
\hline 9/28/11 18:40 & BAQCTB-W-28825 & TB & QC & - & 6633 & $9 / 29 / 11$ & $\begin{array}{l}\text { Trip blank sent to the AGEM Laboratory for VOCs } \\
\text { analyses with water samples listed on chain of } \\
\text { custody forms (COCs) } 6631 \text { and } 6633 \text {. }\end{array}$ \\
\hline 9/28/11 18:56 & BAMW10S-W-28811 & MW & MW10S & $93-103$ & 6631 & $9 / 28 / 11$ & $\begin{array}{l}\text { Depth to water }=74.10 \mathrm{ft} \text {. Depth of } 2 \text {-in. well }=103 \mathrm{ft} \text {. } \\
\text { Sample collected by using low-flow bladder pump } \\
\text { after purging of } 6 \mathrm{~L} \text {. Pump intake positioned at } 98 \mathrm{ft} \text {. }\end{array}$ \\
\hline 9/28/11 18:59 & BAMW4D-W-28808 & MW & MW4D & $98.38-118.22$ & 6631 & $9 / 28 / 11$ & $\begin{array}{l}\text { Depth to water }=95.31 \mathrm{ft} \text {. Depth of } 2 \text {-in. well }= \\
118.22 \mathrm{ft} \text {. Sample collected by using low-flow bladder } \\
\text { pump after purging of } 5 \mathrm{~L} \text {. Pump intake positioned at } \\
108.30 \mathrm{ft} \text {. }\end{array}$ \\
\hline 9/28/11 19:19 & BAPWS3-W-28819 & PW & PWS3 & 160 & 6631 & $9 / 28 / 11$ & $\begin{array}{l}\text { Well converted to run on demand. Sampled from tap } \\
\text { after purging for } 5 \text { min. }\end{array}$ \\
\hline 9/28/11 19:30 & BAPWS2-W-28818 & PW & PWS2 & 155 & 6631 & $9 / 28 / 11$ & $\begin{array}{l}\text { Well runs when the water tower needs replenishing. } \\
\text { Sampled from tap after purging for } 5 \text { min. }\end{array}$ \\
\hline 9/28/11 20:00 & BAMW5-W-28809 & MW & MW5 & $110-120$ & 6631 & $9 / 28 / 11$ & $\begin{array}{l}\text { Depth to water }=96.40 \mathrm{ft} \text {. Depth of } 2 \text {-in. well }=120 \mathrm{ft} \text {. } \\
\text { Sample collected by using low-flow bladder pump } \\
\text { after purging of } 6.5 \mathrm{~L} \text {. Pump intake positioned at } \\
115 \mathrm{ft} \text {. }\end{array}$ \\
\hline 9/29/11 9:50 & BAMW8-W-28810 & MW & MW8 & $110-120$ & 6633 & $9 / 29 / 11$ & $\begin{array}{l}\text { Depth to water }=98.42 \mathrm{ft} \text {. Depth of } 2 \text {-in. well }=120 \mathrm{ft} \text {. } \\
\text { Sample collected by using low-flow bladder pump } \\
\text { after purging of } 4.5 \mathrm{~L} \text {. Pump intake positioned at } \\
115 \mathrm{ft} \text {. }\end{array}$ \\
\hline $12 / 12 / 1115: 24$ & BAPWS2-W-28827 & PW & PWS2 & 155 & 6646 & $12 / 12 / 11$ & $\begin{array}{l}\text { Well runs on demand via sensor in water tower. } \\
\text { Sampled from tap after purging for } 5 \text { min. }\end{array}$ \\
\hline $12 / 12 / 1115: 28$ & BAPWS3-W-28828 & PW & PWS3 & 160 & 6646 & $12 / 12 / 11$ & $\begin{array}{l}\text { Well runs on demand via sensor in water tower. } \\
\text { Sampled from tap after purging for } 5 \text { min. }\end{array}$ \\
\hline $12 / 12 / 1117: 30$ & BAQCTB-W-28829b & TB & QC & - & 6646 & $12 / 12 / 11$ & $\begin{array}{l}\text { Trip blank sent to the AGEM Laboratory for VOC } \\
\text { analyses with water samples listed on COC } 6646 \text {. }\end{array}$ \\
\hline
\end{tabular}

a Sample types: FB, field blank; MW, monitoring well; PW, public water supply well; RI, rinsate; TB, trip blank.

b Quality control sample. 
TABLE A.2 Field measurements for groundwater samples, 2006-2011.

\begin{tabular}{|c|c|c|c|c|c|c|c|}
\hline Well & $\begin{array}{l}\text { Screen Interval } \\
\quad(\mathrm{ft} B G \mathrm{~L})\end{array}$ & $\begin{array}{l}\text { Sample } \\
\text { Date }\end{array}$ & $\begin{array}{c}\text { Temperature } \\
\left({ }^{\circ} \mathrm{C}\right)\end{array}$ & $\mathrm{pH}$ & $\begin{array}{l}\text { Conductivity } \\
(\mu \mathrm{S} / \mathrm{cm})\end{array}$ & $\begin{array}{c}\text { Dissolved } \\
\text { Oxygen } \\
(\mathrm{mg} / \mathrm{L})\end{array}$ & $\begin{array}{r}\text { Oxyg } \\
\text { Reduct } \\
\text { Potent } \\
\text { (mV }\end{array}$ \\
\hline \multicolumn{8}{|c|}{ Previously existing KDHE monitoring wells } \\
\hline \multirow[t]{11}{*}{ MW1S } & 13.3-23.3 & $7 / 19 / 06^{a}$ & - & - & - & - & - \\
\hline & & $4 / 4 / 07^{a}$ & - & - & - & - & - \\
\hline & & $11 / 18 / 07^{a}$ & - & - & - & - & - \\
\hline & & $3 / 4 / 08^{a}$ & - & - & - & - & - \\
\hline & & $7 / 9 / 08^{a}$ & - & - & - & - & - \\
\hline & & $10 / 22 / 08^{a}$ & - & - & - & - & - \\
\hline & & $3 / 4 / 09^{a}$ & - & - & - & - & - \\
\hline & & 6/17/09a & - & - & - & - & - \\
\hline & & 9/30/09a & - & - & - & - & - \\
\hline & & $3 / 31 / 10^{a}$ & - & - & - & - & - \\
\hline & & $9 / 17 / 10^{a}$ & - & - & - & - & - \\
\hline \multirow[t]{12}{*}{ MW1D } & $139.85-159.4$ & $7 / 19 / 06$ & 22.8 & 7.15 & 945 & - & - \\
\hline & & 4/4/07 & 15.7 & 6.30 & 855 & - & - \\
\hline & & $11 / 18 / 07$ & 12.7 & 7.62 & 712 & - & - \\
\hline & & $3 / 4 / 08$ & 5.5 & 7.22 & 1167 & 11.6 & 244 \\
\hline & & 7/9/08 & 18.1 & 7.05 & 992 & 16.2 & 98 \\
\hline & & $10 / 22 / 08$ & 12.6 & 7.07 & 937 & 9.0 & 108 \\
\hline & & 3/4/09 & 13.8 & 7.07 & 962 & 8.9 & 253 \\
\hline & & $6 / 17 / 09$ & 23.3 & 7.07 & 1021 & 5.4 & 106 \\
\hline & & 9/30/09 & 16.3 & 6.87 & 1007 & 6.7 & 268 \\
\hline & & $3 / 31 / 10$ & 16.6 & 7.08 & 760 & 6.6 & -25 \\
\hline & & 9/17/10 & 17.5 & 7.14 & 851 & 8.2 & 160 \\
\hline & & $9 / 28 / 11$ & 18.1 & 7.47 & 799 & 5.8 & 102 \\
\hline \multirow[t]{12}{*}{ MW2D } & 133.26-152.93 & 7/19/06 & 24.7 & 7.72 & 946 & - & - \\
\hline & & 4/4/07 & 15.1 & 6.32 & 887 & - & - \\
\hline & & $11 / 18 / 07$ & 12.1 & 6.96 & 1448 & - & - \\
\hline & & $3 / 7 / 08$ & 6.5 & 7.22 & 1198 & 4.6 & 197 \\
\hline & & 7/10/08 & 18.4 & 6.91 & 1163 & 5.0 & 155 \\
\hline & & $10 / 22 / 08$ & 11.6 & 7.07 & 931 & 6.2 & 132 \\
\hline & & 3/4/09 & 14.5 & 7.06 & 1126 & 5.6 & 243 \\
\hline & & 6/18/09 & 18.7 & 6.97 & 1235 & 3.6 & 116 \\
\hline & & $9 / 30 / 09$ & 17.0 & 6.15 & 1196 & 3.4 & 25 \\
\hline & & $3 / 31 / 10$ & 16.2 & 7.09 & 827 & 5.2 & -37 \\
\hline & & $9 / 17 / 10$ & 20.1 & 7.13 & 945 & 5.0 & 150 \\
\hline & & $9 / 28 / 11$ & 18.6 & 7.72 & 988 & 12.4 & 297 \\
\hline \multirow[t]{11}{*}{ MW3D } & $133.02-152.73$ & $7 / 19 / 06$ & 23.0 & 7.06 & 976 & - & - \\
\hline & & $4 / 4 / 07$ & 15.6 & 6.37 & 989 & - & - \\
\hline & & $11 / 19 / 07$ & 10.5 & 7.16 & 1093 & - & - \\
\hline & & 3/7/08 & 8.2 & 7.09 & 1195 & 5.3 & 255 \\
\hline & & $7 / 10 / 08$ & 19.8 & 6.99 & 1177 & 13.8 & 110 \\
\hline & & $10 / 22 / 08$ & 13.5 & 7.01 & 1238 & 4.5 & 84 \\
\hline & & 3/4/09 & 12.8 & 7.08 & 1062 & 7.0 & 210 \\
\hline & & $6 / 17 / 09$ & 18.5 & 6.65 & 1038 & 5.9 & 110 \\
\hline & & 9/30/09 & 15.2 & 6.87 & 1057 & 6.0 & 209 \\
\hline & & $4 / 1 / 10$ & 18.2 & 7.11 & 789 & 5.6 & -60 \\
\hline & & $9 / 17 / 10$ & 20.0 & 7.00 & 953 & 4.9 & 77 \\
\hline
\end{tabular}


TABLE A.2 (Cont.)

\begin{tabular}{|c|c|c|c|c|c|c|c|}
\hline Well & $\begin{array}{l}\text { Screen Interval } \\
\quad(\mathrm{ft} B G L)\end{array}$ & $\begin{array}{l}\text { Sample } \\
\text { Date }\end{array}$ & $\begin{array}{c}\text { Temperature } \\
\left({ }^{\circ} \mathrm{C}\right)\end{array}$ & $\mathrm{pH}$ & $\begin{array}{l}\text { Conductivity } \\
\qquad(\mu \mathrm{S} / \mathrm{cm})\end{array}$ & $\begin{array}{c}\text { Dissolved } \\
\text { Oxygen } \\
(\mathrm{mg} / \mathrm{L})\end{array}$ & $\begin{array}{r}\text { Reduc } \\
\text { Poten } \\
(\mathrm{mV}\end{array}$ \\
\hline \multicolumn{8}{|c|}{ Previously existing KDHE monitoring wells (cont.) } \\
\hline \multirow[t]{12}{*}{ MW4D } & $98.38-118.22$ & $7 / 20 / 06$ & 23.5 & 6.26 & 968 & - & - \\
\hline & & $4 / 6 / 07$ & 11.3 & 6.21 & 1018 & - & - \\
\hline & & $11 / 19 / 07$ & 15.7 & 6.98 & 1022 & - & - \\
\hline & & $3 / 9 / 08$ & 11.5 & 7.14 & 859 & 6.6 & 201 \\
\hline & & $7 / 12 / 08$ & 14.4 & 6.94 & 1001 & 6.8 & 149 \\
\hline & & $10 / 23 / 08$ & 13.0 & 7.02 & 973 & 5.6 & 94 \\
\hline & & $3 / 5 / 09$ & 15.9 & 7.61 & 1402 & 4.0 & 17 \\
\hline & & $6 / 18 / 09$ & 18.5 & 7.03 & 975 & 5.7 & 127 \\
\hline & & 9/30/09 & 17.3 & 6.85 & 925 & 7.9 & 150 \\
\hline & & $3 / 31 / 10$ & 15.1 & 7.09 & 785 & 7.2 & 22 \\
\hline & & 9/17/10 & 16.5 & 7.01 & 900 & 7.2 & 259 \\
\hline & & $9 / 28 / 11$ & 11.6 & 8.09 & 665 & 7.2 & 186 \\
\hline \multicolumn{8}{|c|}{ CCC/USDA wells installed during 2006-2007 investigation } \\
\hline \multirow[t]{11}{*}{ MW5 } & $110-120$ & $4 / 6 / 07$ & 13.9 & 6.17 & 1705 & - & - \\
\hline & & $11 / 19 / 07$ & 15.2 & 6.74 & 3070 & - & - \\
\hline & & $3 / 8 / 08$ & 9.9 & 6.76 & 2770 & 0.7 & 123 \\
\hline & & $7 / 11 / 08$ & 18.8 & 6.66 & 2930 & 1.3 & 37 \\
\hline & & $10 / 23 / 08$ & 12.8 & 6.78 & 2384 & 0.7 & 20 \\
\hline & & $3 / 5 / 09$ & 15.9 & 6.86 & 2146 & 2.4 & 56 \\
\hline & & 6/19/09 & 16.4 & 6.71 & 2292 & 1.0 & 45 \\
\hline & & 9/30/09 & 16.1 & 6.66 & 1780 & 3.1 & 72 \\
\hline & & $3 / 30 / 10$ & 15.2 & 6.58 & 907 & 4.7 & 17 \\
\hline & & $9 / 17 / 10$ & 17.2 & 6.96 & 1093 & 4.0 & -20 \\
\hline & & $9 / 28 / 11$ & 14.0 & 7.46 & 1049 & 4.1 & 53 \\
\hline \multirow[t]{10}{*}{ MW6S } & $90.5-100.5$ & $4 / 4 / 07^{a}$ & - & - & - & - & - \\
\hline & & $11 / 19 / 07$ & 12.0 & 7.60 & 723 & - & - \\
\hline & & 3/8/08 & 4.7 & 7.77 & 673 & 6.7 & 272 \\
\hline & & $7 / 11 / 08$ & 28.2 & 7.61 & 753 & 9.9 & 92 \\
\hline & & $10 / 23 / 08$ & 11.9 & 11.17 & 582 & 5.1 & 91 \\
\hline & & $3 / 5 / 09$ & 13.9 & 7.88 & 603 & 5.2 & 18 \\
\hline & & 6/18/09 & 19.5 & 7.70 & 698 & 4.5 & 27 \\
\hline & & 10/1/09 & 14.6 & 6.30 & 618 & 5.8 & 88 \\
\hline & & $3 / 31 / 10$ & 16.4 & 7.52 & 584 & 5.8 & -25 \\
\hline & & $9 / 18 / 10$ & 15.8 & 11.16 & 568 & 2.3 & 35 \\
\hline \multirow[t]{10}{*}{ MW6D } & $105-115$ & $4 / 5 / 07$ & 6.2 & 6.11 & 936 & - & - \\
\hline & & $11 / 19 / 07$ & 13.6 & 7.00 & 1103 & - & - \\
\hline & & $3 / 8 / 08$ & 9.1 & 7.15 & 908 & 5.6 & 241 \\
\hline & & 7/11/08 & 19.8 & 7.05 & 999 & 12.8 & 100 \\
\hline & & $10 / 23 / 08$ & 12.0 & 7.18 & 957 & 6.3 & 128 \\
\hline & & 3/5/09 & 13.4 & 7.22 & 903 & 3.1 & 21 \\
\hline & & 6/18/09 & 19.0 & 6.86 & 992 & 4.4 & 114 \\
\hline & & $10 / 1 / 09$ & 16.0 & 6.41 & 910 & 4.2 & 98 \\
\hline & & $3 / 31 / 10$ & 17.6 & 7.10 & 821 & 5.0 & -2 \\
\hline & & $9 / 18 / 10$ & 15.9 & 7.06 & 981 & 4.9 & 147 \\
\hline
\end{tabular}


TABLE A.2 (Cont.)

\begin{tabular}{|c|c|c|c|c|c|c|c|}
\hline Well & $\begin{array}{l}\text { Screen Interval } \\
\quad(\mathrm{ft} B G L)\end{array}$ & $\begin{array}{l}\text { Sample } \\
\text { Date }\end{array}$ & $\begin{array}{l}\text { Temperature } \\
\left({ }^{\circ} \mathrm{C}\right)\end{array}$ & $\mathrm{pH}$ & $\begin{array}{l}\text { Conductivity } \\
(\mu S / \mathrm{cm})\end{array}$ & $\begin{array}{c}\text { Dissolved } \\
\text { Oxygen } \\
(\mathrm{mg} / \mathrm{L})\end{array}$ & $\begin{array}{l}\text { Oxygen } \\
\text { Reduction } \\
\text { Potential } \\
(\mathrm{mV})\end{array}$ \\
\hline \multicolumn{8}{|c|}{ CCC/USDA wells installed during 2006-2007 investigation (cont.) } \\
\hline \multirow[t]{10}{*}{ MW7 } & $116-126$ & $4 / 6 / 07$ & 14.1 & 6.30 & 1051 & - & - \\
\hline & & 11/19/07 & 14.6 & 7.16 & 890 & - & - \\
\hline & & $3 / 9 / 08$ & 13.1 & 7.10 & 1068 & 4.2 & 186 \\
\hline & & $7 / 12 / 08$ & 14.4 & 6.95 & 1238 & 4.4 & 98 \\
\hline & & $10 / 23 / 08$ & 12.7 & 7.16 & 1191 & 8.6 & 122 \\
\hline & & $3 / 5 / 09$ & 15.3 & 7.11 & 1141 & 9.9 & 126 \\
\hline & & 6/19/09 & 16.3 & 7.05 & 1174 & 4.3 & 48 \\
\hline & & $9 / 30 / 09$ & 16.2 & 6.86 & 1132 & 8.2 & 216 \\
\hline & & $3 / 30 / 10$ & 16.4 & 7.04 & 923 & 7.3 & -48 \\
\hline & & $9 / 17 / 10$ & 17.2 & 6.88 & 1149 & 4.2 & 104 \\
\hline \multirow[t]{11}{*}{ MW8 } & $110-120$ & $4 / 6 / 07$ & 12.1 & 6.23 & 974 & - & - \\
\hline & & $11 / 19 / 07$ & 14.6 & 7.03 & 909 & - & - \\
\hline & & $3 / 10 / 08$ & 13.1 & 7.09 & 961 & 6.7 & 182 \\
\hline & & $7 / 11 / 08$ & 18.6 & 6.38 & 1049 & 6.2 & 152 \\
\hline & & 10/23/08 & 12.9 & 7.06 & 948 & 8.7 & 97 \\
\hline & & $3 / 5 / 09$ & 16.2 & 7.12 & 985 & 9.4 & 165 \\
\hline & & $6 / 19 / 09$ & 18.0 & 7.07 & 972 & 6.7 & 102 \\
\hline & & $9 / 30 / 09$ & 16.7 & 6.15 & 889 & 6.2 & 33 \\
\hline & & $3 / 31 / 10$ & 14.0 & 7.07 & 815 & 7.5 & 23 \\
\hline & & $9 / 17 / 10$ & 17.4 & 7.05 & 940 & 6.9 & 231 \\
\hline & & $9 / 29 / 11$ & 15.8 & 7.69 & 801 & 7.3 & 253 \\
\hline \multirow[t]{10}{*}{ MW9 } & $100-110$ & $4 / 5 / 07$ & 12.9 & 6.20 & 976 & - & - \\
\hline & & $11 / 19 / 07$ & 16.5 & 7.21 & 1066 & - & - \\
\hline & & $3 / 9 / 08$ & 11.2 & 7.07 & 928 & 5.8 & 239 \\
\hline & & $7 / 11 / 08$ & 17.7 & 6.58 & 1010 & 5.6 & 189 \\
\hline & & $10 / 24 / 08$ & 13.0 & 7.06 & 888 & 5.4 & 79 \\
\hline & & 3/5/09 & 16.2 & 7.10 & 939 & 8.4 & 173 \\
\hline & & $6 / 17 / 09$ & 15.9 & 6.87 & 907 & 5.6 & 146 \\
\hline & & 9/29/09 & 13.9 & 6.79 & 871 & 7.8 & 135 \\
\hline & & $3 / 31 / 10$ & 15.4 & 6.88 & 754 & 7.3 & 123 \\
\hline & & $9 / 18 / 10$ & 14.7 & 7.14 & 879 & 7.2 & 188 \\
\hline \multirow[t]{11}{*}{ MW10S } & $93-103$ & $4 / 6 / 07$ & 13.2 & 6.36 & 1004 & - & - \\
\hline & & 11/19/07 & 14.5 & 7.22 & 942 & - & - \\
\hline & & $3 / 10 / 08$ & 12.7 & 7.08 & 912 & 5.2 & 176 \\
\hline & & $7 / 11 / 08$ & 17.3 & 6.91 & 975 & 12.8 & 119 \\
\hline & & $10 / 23 / 08$ & 13.4 & 7.08 & 913 & 5.4 & 78 \\
\hline & & $3 / 5 / 09$ & 15.3 & 7.25 & 895 & 2.7 & 13 \\
\hline & & $6 / 19 / 09$ & 16.0 & 7.12 & 925 & 5.4 & 104 \\
\hline & & $9 / 30 / 09$ & 15.8 & 6.58 & 829 & 4.6 & 31 \\
\hline & & $3 / 30 / 10$ & 15.3 & 7.08 & 776 & 6.9 & -25 \\
\hline & & 9/17/10 & 17.2 & 7.02 & 865 & 5.2 & 164 \\
\hline & & $9 / 28 / 11$ & 14.6 & 7.44 & 677 & 5.6 & 125 \\
\hline
\end{tabular}


TABLE A.2 (Cont.)

\begin{tabular}{|c|c|c|c|c|c|c|c|}
\hline Well & $\begin{array}{l}\text { Screen Interval } \\
\quad(\mathrm{ft} B G L)\end{array}$ & $\begin{array}{l}\text { Sample } \\
\text { Date }\end{array}$ & $\begin{array}{l}\text { Temperature } \\
\left({ }^{\circ} \mathrm{C}\right)\end{array}$ & $\mathrm{pH}$ & $\begin{array}{l}\text { Conductivity } \\
(\mu S / \mathrm{cm})\end{array}$ & $\begin{array}{c}\text { Dissolved } \\
\text { Oxygen } \\
(\mathrm{mg} / \mathrm{L})\end{array}$ & $\begin{array}{l}\text { Oxygen } \\
\text { Reduction } \\
\text { Potential } \\
(\mathrm{mV})\end{array}$ \\
\hline \multicolumn{8}{|c|}{ CCC/USDA wells installed during 2006-2007 investigation (cont.) } \\
\hline \multirow[t]{11}{*}{ MW10D } & $115-125$ & $4 / 6 / 07$ & 12.1 & 6.21 & 992 & - & - \\
\hline & & $11 / 19 / 07$ & 14.5 & 7.42 & 1175 & - & - \\
\hline & & $3 / 9 / 08$ & 13.7 & 7.01 & 1024 & 5.1 & 236 \\
\hline & & 7/11/08 & 17.4 & 6.78 & 1090 & 12.6 & 117 \\
\hline & & $10 / 23 / 08$ & 13.5 & 7.01 & 1000 & 5.0 & 93 \\
\hline & & $3 / 5 / 09$ & 15.9 & 7.20 & 969 & 4.4 & 12 \\
\hline & & 6/19/09 & 16.9 & 7.08 & 1035 & 5.7 & 109 \\
\hline & & $9 / 30 / 09$ & 15.8 & 6.62 & 922 & 5.5 & 32 \\
\hline & & $3 / 30 / 10$ & 15.1 & 7.00 & 835 & 6.4 & -34 \\
\hline & & $9 / 17 / 10$ & 17.6 & 6.97 & 955 & 5.7 & 204 \\
\hline & & $9 / 28 / 11$ & 15.7 & 7.36 & 735 & 6.1 & 138 \\
\hline \multirow[t]{10}{*}{ MW11S } & $40-50$ & $4 / 4 / 07$ & 12.8 & 6.14 & 1027 & - & - \\
\hline & & $11 / 19 / 07$ & 11.2 & 7.15 & 1174 & - & - \\
\hline & & $3 / 5 / 08$ & 9.4 & 6.81 & 1122 & 2.3 & 241 \\
\hline & & $7 / 10 / 08$ & 19.5 & 6.47 & 1224 & 1.9 & 166 \\
\hline & & $10 / 23 / 08$ & 10.2 & 6.99 & 1085 & 5.8 & 146 \\
\hline & & 3/4/09 & 14.5 & 7.11 & 1186 & 4.3 & 37 \\
\hline & & $6 / 19 / 09$ & 15.0 & 6.81 & 1159 & 3.1 & 173 \\
\hline & & 10/1/09 & 15.9 & 6.67 & 1114 & 2.2 & 203 \\
\hline & & $3 / 31 / 10$ & 18.0 & 6.81 & 958 & 6.1 & 84 \\
\hline & & $9 / 18 / 10$ & 15.8 & 6.98 & 1178 & 2.0 & 185 \\
\hline \multirow[t]{10}{*}{ MW11M } & $90-100$ & $4 / 5 / 07$ & 7.5 & 7.60 & 1097 & - & - \\
\hline & & $11 / 19 / 07$ & 11.9 & 7.17 & 1144 & - & - \\
\hline & & $3 / 6 / 08$ & 10.8 & 7.06 & 997 & 2.7 & 254 \\
\hline & & $7 / 10 / 08$ & 31.9 & 7.08 & 1124 & 3.9 & 149 \\
\hline & & $10 / 23 / 08$ & 12.4 & 7.06 & 962 & 3.2 & 116 \\
\hline & & $3 / 4 / 09$ & 13.6 & 7.33 & 910 & 4.9 & 28 \\
\hline & & 6/19/09 & 14.7 & 6.92 & 973 & 4.8 & 185 \\
\hline & & 10/1/09 & 14.5 & 6.85 & 919 & 6.7 & 153 \\
\hline & & $3 / 31 / 10$ & 17.4 & 6.96 & 742 & 5.5 & 67 \\
\hline & & 9/18/10 & 16.0 & 7.22 & 846 & 8.8 & 174 \\
\hline \multirow[t]{10}{*}{ MW11D } & $125-135$ & $4 / 4 / 07$ & 13.8 & 6.18 & 990 & - & - \\
\hline & & $11 / 19 / 07$ & 13.1 & 7.22 & 987 & - & - \\
\hline & & $3 / 5 / 08$ & 6.0 & 7.06 & 872 & 6.9 & 252 \\
\hline & & $7 / 10 / 08$ & 17.5 & 6.25 & 957 & 7.1 & 177 \\
\hline & & $10 / 23 / 08$ & 12.3 & 7.11 & 863 & 8.7 & 123 \\
\hline & & 3/4/09 & 14.3 & 7.23 & 848 & 4.5 & 27 \\
\hline & & $6 / 19 / 09$ & 17.4 & 6.96 & 885 & 5.9 & 131 \\
\hline & & $10 / 1 / 09$ & 14.7 & 6.85 & 854 & 6.9 & 154 \\
\hline & & 4/1/10 & 17.4 & 6.91 & 750 & 7.4 & 99 \\
\hline & & $9 / 18 / 10$ & 15.8 & 7.11 & 871 & 6.5 & 189 \\
\hline
\end{tabular}


TABLE A.2 (Cont.)

\begin{tabular}{|c|c|c|c|c|c|c|c|}
\hline Well & $\begin{array}{l}\text { Screen Interval } \\
\quad(\mathrm{ft} B G L)\end{array}$ & $\begin{array}{l}\text { Sample } \\
\text { Date }\end{array}$ & $\begin{array}{c}\text { Temperature } \\
\left({ }^{\circ} \mathrm{C}\right)\end{array}$ & $\mathrm{pH}$ & $\begin{array}{l}\text { Conductivity } \\
(\mu \mathrm{S} / \mathrm{cm})\end{array}$ & $\begin{array}{c}\text { Dissolved } \\
\text { Oxygen } \\
(\mathrm{mg} / \mathrm{L})\end{array}$ & $\begin{array}{r}\text { Oxyg } \\
\text { Reduc } \\
\text { Poten } \\
\text { (mV }\end{array}$ \\
\hline \multicolumn{8}{|c|}{ CCC/USDA wells installed during 2006-2007 investigation (cont.) } \\
\hline \multirow[t]{10}{*}{ MW12S } & $43-53$ & $4 / 5 / 07^{a}$ & - & - & - & - & - \\
\hline & & $11 / 19 / 07^{a}$ & - & - & - & - & - \\
\hline & & $3 / 10 / 08^{a}$ & - & - & - & - & - \\
\hline & & $7 / 10 / 08^{a}$ & - & - & - & - & - \\
\hline & & $10 / 22 / 08^{a}$ & - & - & - & - & - \\
\hline & & $3 / 4 / 09^{a}$ & - & - & - & - & - \\
\hline & & 6/19/09 & 20.5 & 7.00 & 1436 & - & - \\
\hline & & $10 / 1 / 09^{a}$ & - & - & - & - & - \\
\hline & & $3 / 31 / 10^{a}$ & - & - & - & - & - \\
\hline & & $9 / 18 / 10^{a}$ & - & - & - & - & - \\
\hline \multirow[t]{11}{*}{ MW12M } & $90-100$ & $4 / 5 / 07$ & 12.6 & 6.42 & 867 & - & - \\
\hline & & $11 / 19 / 07$ & 14.9 & 7.13 & 835 & - & - \\
\hline & & 3/10/08 & 12.6 & 7.13 & 665 & 1.8 & 212 \\
\hline & & $7 / 10 / 08$ & 16.9 & 7.09 & 878 & 8.2 & 87 \\
\hline & & $10 / 22 / 08$ & 12.2 & 7.20 & 785 & 8.9 & 126 \\
\hline & & 3/4/09 & 14.1 & 7.24 & 851 & 2.2 & 20 \\
\hline & & $6 / 19 / 09$ & 17.0 & 6.99 & 856 & 2.3 & 112 \\
\hline & & $10 / 1 / 09$ & 14.4 & 6.81 & 840 & 3.4 & 52 \\
\hline & & $3 / 31 / 10$ & 17.5 & 7.05 & 334 & 0.4 & 36 \\
\hline & & $9 / 18 / 10$ & 15.8 & 7.13 & 649 & 0.2 & -61 \\
\hline & & $9 / 28 / 11$ & 14.8 & 7.82 & 648 & 1.8 & 39 \\
\hline \multirow[t]{10}{*}{ MW12D } & $115-125$ & $4 / 5 / 07$ & 14.0 & 6.36 & 930 & - & - \\
\hline & & $11 / 18 / 07$ & 15.6 & 6.95 & 571 & - & - \\
\hline & & $3 / 9 / 08$ & 8.8 & 7.13 & 881 & 5.3 & 237 \\
\hline & & $7 / 11 / 08$ & 19.9 & 6.01 & 987 & 4.7 & 197 \\
\hline & & $10 / 22 / 08$ & 12.3 & 7.09 & 873 & 8.0 & 136 \\
\hline & & 3/4/09 & 14.4 & 7.25 & 923 & 4.1 & 21 \\
\hline & & $6 / 19 / 09$ & 16.4 & 6.96 & 895 & 5.1 & 150 \\
\hline & & 10/1/09 & 15.8 & 6.85 & 869 & 6.7 & 154 \\
\hline & & $3 / 31 / 10$ & 16.6 & 6.89 & 753 & 6.6 & 103 \\
\hline & & $9 / 18 / 10$ & 15.5 & 6.97 & 872 & 6.2 & 139 \\
\hline \multirow[t]{11}{*}{ MW13S } & $112-122$ & $4 / 5 / 07$ & 9.8 & 6.42 & 946 & - & - \\
\hline & & $11 / 19 / 07$ & 16.5 & 7.21 & 893 & - & - \\
\hline & & $3 / 10 / 08$ & 12.2 & 7.13 & 810 & 6.2 & 199 \\
\hline & & 7/9/08 & 17.4 & 6.99 & 875 & 7.7 & 116 \\
\hline & & $10 / 22 / 08$ & 13.5 & 7.08 & 793 & 5.1 & 100 \\
\hline & & $3 / 4 / 09$ & 13.8 & 7.23 & 818 & 4.4 & 29 \\
\hline & & 6/18/09 & 17.6 & 7.04 & 803 & 6.1 & 104 \\
\hline & & $9 / 30 / 09$ & 15.4 & 5.74 & 721 & 6.1 & 177 \\
\hline & & $4 / 1 / 10$ & 15.0 & 7.14 & 667 & 7.5 & 64 \\
\hline & & $9 / 18 / 10$ & 16.7 & 7.42 & 772 & 12.9 & 186 \\
\hline & & $9 / 28 / 11$ & 16.8 & 7.60 & 633 & 6.8 & 154 \\
\hline
\end{tabular}


TABLE A.2 (Cont.)

\begin{tabular}{|c|c|c|c|c|c|c|c|}
\hline Well & $\begin{array}{l}\text { Screen Interval } \\
\quad(\mathrm{ft} B G L)\end{array}$ & $\begin{array}{l}\text { Sample } \\
\text { Date }\end{array}$ & $\begin{array}{l}\text { Temperature } \\
\left({ }^{\circ} \mathrm{C}\right)\end{array}$ & $\mathrm{pH}$ & $\begin{array}{l}\text { Conductivity } \\
(\mu \mathrm{S} / \mathrm{cm})\end{array}$ & $\begin{array}{c}\text { Dissolved } \\
\text { Oxygen } \\
(\mathrm{mg} / \mathrm{L})\end{array}$ & $\begin{array}{l}\text { Oxygen } \\
\text { Reduction } \\
\text { Potential } \\
(\mathrm{mV})\end{array}$ \\
\hline \multicolumn{8}{|c|}{ CCC/USDA wells installed during 2006-2007 investigation (cont.) } \\
\hline \multirow[t]{11}{*}{ MW13D } & $127-137$ & $4 / 5 / 07$ & 14.9 & 6.25 & 397 & - & - \\
\hline & & $11 / 19 / 07$ & 17.0 & 7.00 & 763 & - & - \\
\hline & & $3 / 9 / 08$ & 13.1 & 7.09 & 758 & 6.0 & 213 \\
\hline & & $7 / 9 / 08$ & 18.6 & 7.07 & 848 & 18.1 & 57 \\
\hline & & $10 / 22 / 08$ & 13.3 & 7.06 & 824 & 5.3 & 98 \\
\hline & & $3 / 4 / 09$ & 14.5 & 7.19 & 833 & 4.0 & 25 \\
\hline & & 6/18/09 & 17.5 & 6.93 & 828 & 5.6 & 117 \\
\hline & & $9 / 30 / 09$ & 17.4 & 5.99 & 706 & 5.6 & 142 \\
\hline & & $4 / 1 / 10$ & 16.3 & 7.09 & 694 & 6.6 & 21 \\
\hline & & $9 / 18 / 10$ & 17.3 & 7.14 & 803 & 6.3 & 189 \\
\hline & & $9 / 28 / 11$ & 18.0 & 7.59 & 687 & 16.1 & 137 \\
\hline \multirow[t]{11}{*}{ MW14S } & $108-118$ & $4 / 4 / 07$ & 13.4 & 6.50 & 704 & - & - \\
\hline & & $11 / 18 / 07$ & 12.9 & 7.26 & 966 & - & - \\
\hline & & $3 / 8 / 08$ & 13.2 & 7.20 & 729 & 6.6 & 208 \\
\hline & & 7/10/08 & 17.4 & 7.16 & 775 & 16.4 & 87 \\
\hline & & $10 / 22 / 08$ & 13.2 & 7.11 & 716 & 5.8 & 90 \\
\hline & & $3 / 4 / 09$ & 13.8 & 7.15 & 736 & 9.0 & 268 \\
\hline & & $6 / 18 / 09$ & 17.5 & 7.01 & 742 & 5.2 & 97 \\
\hline & & $10 / 1 / 09$ & 16.0 & 6.25 & 663 & 5.8 & 99 \\
\hline & & 4/1/10 & 17.8 & 6.96 & 625 & 7.7 & 73 \\
\hline & & $9 / 18 / 10$ & 16.4 & 7.11 & 705 & 6.8 & 193 \\
\hline & & $9 / 28 / 11$ & 17.2 & 7.62 & 592 & 7.0 & 118 \\
\hline \multirow[t]{10}{*}{ MW14D } & $123-133$ & 4/4/07 & 14.7 & 6.34 & 932 & - & - \\
\hline & & $11 / 18 / 07$ & 13.2 & 7.47 & 739 & - & - \\
\hline & & $3 / 8 / 08$ & 12.0 & 7.06 & 1424 & 2.0 & 282 \\
\hline & & $7 / 10 / 08$ & 17.7 & 7.07 & 1459 & 14.5 & 86 \\
\hline & & $10 / 22 / 08$ & 13.0 & 7.00 & 1212 & 1.4 & 79 \\
\hline & & $3 / 5 / 09$ & 13.6 & 7.06 & 1339 & 2.2 & 69 \\
\hline & & 6/18/09 & 19.8 & 7.04 & 1523 & 2.0 & 46 \\
\hline & & $10 / 1 / 09$ & 15.5 & 6.21 & 1154 & 1.6 & 90 \\
\hline & & $4 / 1 / 10$ & 17.8 & 6.93 & 1189 & 1.8 & 67 \\
\hline & & 9/17/10 & 16.1 & 7.09 & 1162 & 6.5 & 45 \\
\hline \multirow[t]{10}{*}{ MW15S } & $88-98$ & $4 / 4 / 07$ & 13.1 & 8.03 & 854 & - & - \\
\hline & & $11 / 18 / 07$ & 13.9 & NR & 1883 & - & - \\
\hline & & $3 / 10 / 08$ & 12.1 & 8.67 & 697 & 5.5 & 173 \\
\hline & & $7 / 12 / 08$ & 14.0 & 8.88 & 660 & 11.6 & 94 \\
\hline & & $10 / 23 / 08$ & 13.0 & 8.00 & 789 & 5.3 & 67 \\
\hline & & 3/5/09 & 15.7 & 8.78 & 589 & 7.5 & 163 \\
\hline & & $6 / 17 / 09$ & 18.2 & 9.13 & 386 & 4.8 & 62 \\
\hline & & $9 / 29 / 09$ & 15.4 & 7.40 & 786 & 6.9 & 181 \\
\hline & & $3 / 30 / 10$ & 15.7 & 7.81 & 467 & 6.7 & 63 \\
\hline & & $9 / 18 / 10$ & 16.4 & 7.49 & 776 & 5.8 & 127 \\
\hline
\end{tabular}


TABLE A.2 (Cont.)

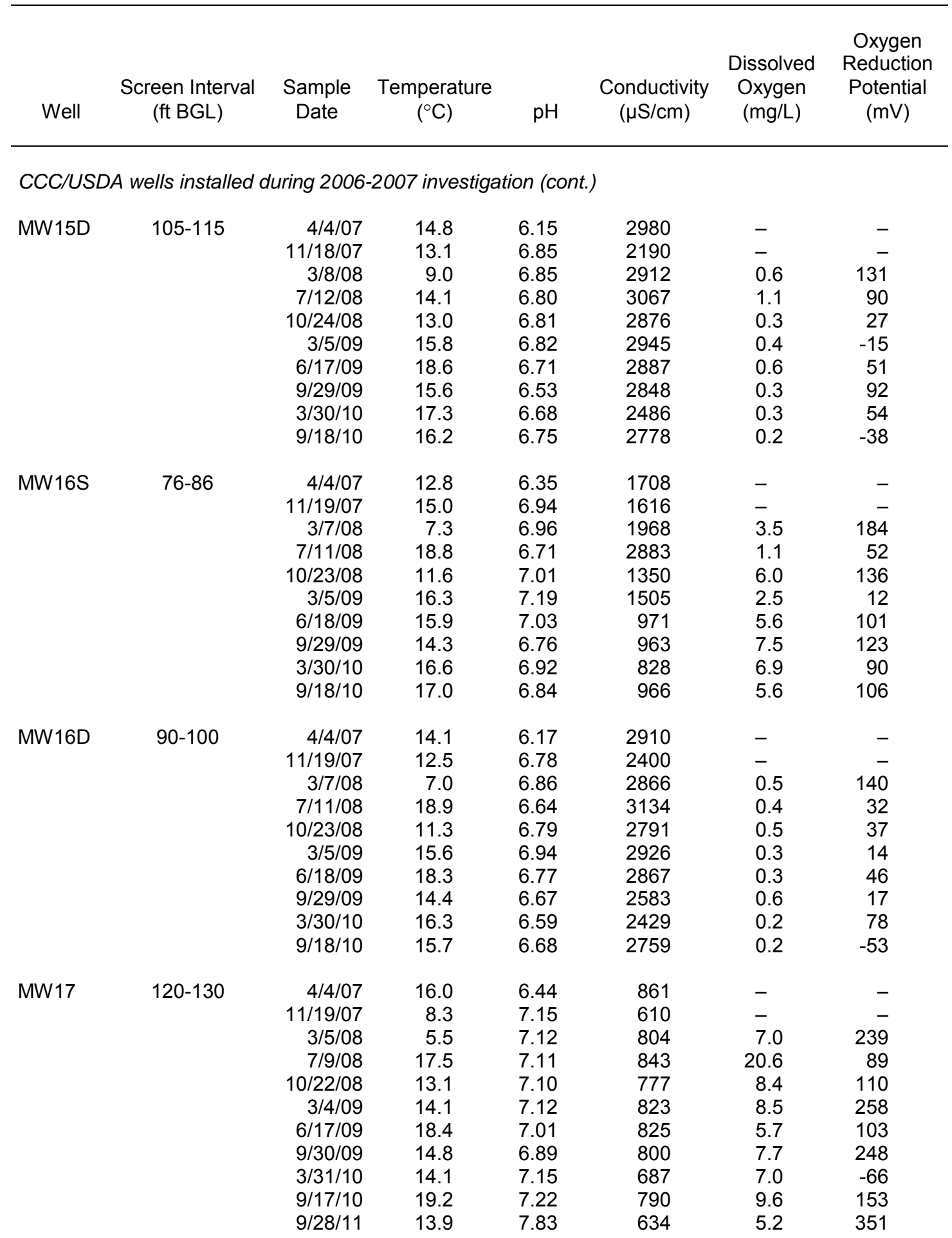


TABLE A.2 (Cont.)

\begin{tabular}{|c|c|c|c|c|c|c|c|}
\hline Well & $\begin{array}{l}\text { Screen Interval } \\
\quad(\mathrm{ft} B G L)\end{array}$ & $\begin{array}{l}\text { Sample } \\
\text { Date }\end{array}$ & $\begin{array}{c}\text { Temperature } \\
\left({ }^{\circ} \mathrm{C}\right)\end{array}$ & $\mathrm{pH}$ & $\begin{array}{l}\text { Conductivity } \\
(\mu \mathrm{S} / \mathrm{cm})\end{array}$ & $\begin{array}{c}\text { Dissolved } \\
\text { Oxygen } \\
\text { (mg/L) }\end{array}$ & $\begin{array}{c}\text { Oxygen } \\
\text { Reduction } \\
\text { Potential } \\
(\mathrm{mV})\end{array}$ \\
\hline \multicolumn{8}{|c|}{ Private wells } \\
\hline Oentrich & 150 & $\begin{array}{r}7 / 20 / 06 \\
8 / 2 / 06 \\
4 / 5 / 07 \\
11 / 19 / 07 \\
3 / 6 / 08 \\
7 / 11 / 08 \\
10 / 23 / 08 \\
3 / 5 / 09 \\
6 / 18 / 09 \\
9 / 30 / 09 \\
4 / 1 / 10 \\
9 / 18 / 10\end{array}$ & $\begin{array}{c}- \\
- \\
\overline{-} \\
12.1 \\
- \\
- \\
- \\
- \\
15.3 \\
15.6 \\
12.8 \\
-\end{array}$ & $\begin{array}{l}- \\
- \\
- \\
8.26 \\
- \\
- \\
- \\
- \\
7.15 \\
7.14 \\
7.41 \\
-\end{array}$ & $\begin{array}{r}- \\
- \\
- \\
1830 \\
- \\
- \\
- \\
- \\
1270 \\
1275 \\
1017 \\
-\end{array}$ & $\begin{array}{l}- \\
- \\
- \\
- \\
- \\
- \\
- \\
- \\
- \\
- \\
- \\
-\end{array}$ & $\begin{array}{l}- \\
- \\
- \\
- \\
- \\
- \\
- \\
- \\
- \\
- \\
- \\
-\end{array}$ \\
\hline Sedivy & 138 & $\begin{array}{l}8 / 22 / 06 \\
9 / 13 / 06\end{array}$ & $-\overline{22.5}$ & $\overline{6} .57$ & $73 \overline{9}$ & $\begin{array}{l}- \\
-\end{array}$ & $\begin{array}{l}- \\
-\end{array}$ \\
\hline Public we & & & & & & & \\
\hline PWS2 & 155 & $\begin{array}{r}11 / 20 / 07 \\
6 / 18 / 09 \\
9 / 30 / 09 \\
12 / 14 / 09 \\
3 / 31 / 10 \\
6 / 17 / 10 \\
9 / 28 / 11 \\
12 / 12 / 11\end{array}$ & $\begin{array}{c}- \\
- \\
- \\
- \\
- \\
- \\
15.2 \\
15.1\end{array}$ & $\begin{array}{l}- \\
- \\
- \\
- \\
- \\
- \\
8.04 \\
7.88\end{array}$ & $\begin{array}{l}955 \\
863 \\
851 \\
857 \\
853 \\
866 \\
694 \\
694\end{array}$ & $\begin{array}{l}- \\
- \\
- \\
- \\
- \\
- \\
- \\
-\end{array}$ & $\begin{array}{l}- \\
- \\
- \\
- \\
- \\
- \\
- \\
-\end{array}$ \\
\hline PWS3 & 160 & $\begin{array}{r}11 / 20 / 07 \\
6 / 18 / 09 \\
9 / 30 / 09 \\
12 / 14 / 09 \\
3 / 31 / 10 \\
6 / 17 / 10 \\
9 / 28 / 11 \\
12 / 12 / 11\end{array}$ & $\begin{array}{l}- \\
- \\
- \\
- \\
- \\
- \\
15.7 \\
15.0\end{array}$ & $\begin{array}{l}- \\
- \\
- \\
- \\
- \\
- \\
7.97 \\
7.83\end{array}$ & $\begin{array}{l}999 \\
867 \\
858 \\
859 \\
867 \\
880 \\
721 \\
718\end{array}$ & $\begin{array}{l}- \\
- \\
- \\
- \\
- \\
- \\
- \\
-\end{array}$ & $\begin{array}{l}- \\
- \\
- \\
- \\
- \\
- \\
- \\
-\end{array}$ \\
\hline
\end{tabular}

a Not sampled (well dry). 


\section{Appendix B:}

Results from the AGEM Laboratory for Dual Analyses of Samples Collected in 2011 and for Quality Control Samples 
TABLE B.1 Analytical results from the AGEM Laboratory for quality control samples collected to monitor sample collection and handling activities in 2011.

\begin{tabular}{|c|c|c|c|c|c|c|c|}
\hline \multirow[b]{2}{*}{$\begin{array}{c}\text { Sample } \\
\text { Date and Time }\end{array}$} & \multirow[b]{2}{*}{ Location } & \multirow[b]{2}{*}{ Sample } & \multirow[b]{2}{*}{$\begin{array}{l}\text { Depth } \\
\text { (ft BGL) }\end{array}$} & \multicolumn{3}{|c|}{ Concentration $(\mu \mathrm{g} / \mathrm{L})$} & \multirow[b]{2}{*}{ Type } \\
\hline & & & & $\begin{array}{c}\text { Carbon } \\
\text { Tetrachloride }\end{array}$ & Chloroform & $\begin{array}{c}\text { Methylene } \\
\text { Chloride }\end{array}$ & \\
\hline 9/28/11 17:19 & MW12M & BAMW12M-W-28813 & $90-100$ & 16 & 5.1 & $N^{a}$ & Primary sample \\
\hline $9 / 28 / 1117: 20$ & MW12M & BAMW12MDUP-W-28821 & $90-100$ & 17 & 5.2 & ND & Replicate sample \\
\hline 9/28/11 10:52 & MW13S & BAMW13S-W-28814 & $112-122$ & 8.1 & $0.7 \mathrm{~J}^{\mathrm{b}}$ & ND & Primary sample \\
\hline 9/28/11 10:52 & MW13S & BAMW13S-W-28814DUP & $112-122$ & 8.2 & $0.7 \mathrm{~J}$ & ND & Duplicate analysis \\
\hline 9/28/11 15:36 & MW1D & BAMW1D-W-28806 & $139.85-159.4$ & ND & ND & ND & Primary sample \\
\hline 9/28/11 15:37 & MW1D & BAMW1DDUP-W-28820 & $139.85-159.4$ & ND & ND & ND & Replicate sample \\
\hline 9/28/11 17:45 & QC & BAQCIR-W-28822 & - & ND & ND & ND & Equipment rinsate \\
\hline 9/28/11 18:03 & QC & BAQCIR-W-28823 & - & ND & ND & ND & Equipment rinsate \\
\hline $9 / 28 / 1118: 34$ & QC & BADIH2O-W-28824 & - & ND & ND & ND & Field blank \\
\hline 9/28/11 18:40 & QC & BAQCTB-W-28825 & - & ND & ND & ND & Trip blank \\
\hline $12 / 12 / 1115: 24$ & PWS2 & BAPWS2-W-28827 & 155 & ND & ND & ND & Primary sample \\
\hline $12 / 12 / 1115: 28$ & PWS2 & BAPWS2-W-28827DUP & 155 & ND & ND & ND & Duplicate analysis \\
\hline 12/12/11 17:30 & QC & BAQCTB-W-28829 & - & ND & ND & ND & Trip blank \\
\hline
\end{tabular}

a ND, contaminant not detected at an instrument detection limit of $0.1 \mu \mathrm{g} / \mathrm{L}$.

b J, estimated concentration below the purge-and-trap method quantitation limit of $1.0 \mu \mathrm{g} / \mathrm{L}$. 
TABLE B.2 Results for verification organic analyses during groundwater monitoring in 2011.

\begin{tabular}{|c|c|c|c|c|c|c|c|c|}
\hline \multirow[b]{3}{*}{ Location } & \multirow[b]{3}{*}{ Sample } & \multirow[b]{3}{*}{$\begin{array}{c}\text { Sample } \\
\text { Date and Time }\end{array}$} & \multicolumn{6}{|c|}{ Concentration $(\mu \mathrm{g} / \mathrm{L})$} \\
\hline & & & \multicolumn{3}{|c|}{ AGEM Laboratory } & \multicolumn{3}{|c|}{ TestAmerica } \\
\hline & & & $\begin{array}{c}\text { Carbon } \\
\text { Tetrachloride }\end{array}$ & Chloroform & $\begin{array}{l}\text { Methylene } \\
\text { Chloride }\end{array}$ & $\begin{array}{c}\text { Carbon } \\
\text { Tetrachloride }\end{array}$ & Chloroform & $\begin{array}{l}\text { Methylene } \\
\text { Chloride }\end{array}$ \\
\hline MW13S & BAMW13S-W-28814 & $9 / 28 / 1110: 52$ & 8.1 & $0.7 \mathrm{~J}$ & $N D^{a}$ & 8.3 & 0.69 & ND \\
\hline MW14S & BAMW14S-W-28816 & $9 / 28 / 1116: 40$ & 3.4 & ND & ND & 2.8 & $0.21 \mathrm{~J}^{\mathrm{b}}$ & ND \\
\hline MW17 & BAMW17-W-28817 & 9/28/11 15:10 & $0.4 \mathrm{~J}$ & ND & ND & $0.33 \mathrm{~J}$ & ND & ND \\
\hline QC & BAQCTB-W-28825 & $9 / 28 / 1118: 40$ & ND & ND & ND & ND & ND & ND \\
\hline
\end{tabular}

a ND, not detected at an instrument detection limit of $0.1 \mu \mathrm{g} / \mathrm{L}$.

b J, estimated concentration below the method quantitation limit of $1.0 \mu \mathrm{g} / \mathrm{L}$ at the AGEM Laboratatory (for modified EPA Method 524.2 ) or 0.5 $\mu \mathrm{g} / \mathrm{L}$ at TestAmerica (for EPA Method SOM01 — trace volatiles). 


\section{Supplement 1:}

\section{Waste Characterization Data}


November 11, 2011

Mr. Travis Kamler

TCW Construction Inc

$141 \mathrm{M}$ Street

Lincoln, NE 68508

RE: Project: KS/MO Waste Water

Pace Project No.: 60109211

Dear Mr. Kamler:

Enclosed are the analytical results for sample(s) received by the laboratory on November 01, 2011.

The results relate only to the samples included in this report. Results reported herein conform to the most current TNI standards and the laboratory's Quality Assurance Manual, where applicable, unless otherwise noted in the body of the report.

If you have any questions concerning this report, please feel free to contact me.

Sincerely,

$$
\text { suedy sigason }
$$

Trudy Gipson

trudy.gipson@pacelabs.com

Project Manager

\author{
Enclosures \\ cc: Mr. David Surgnier
}




\section{CERTIFICATIONS}

Project: $\quad$ KS/MO Waste Water

Pace Project No.: 60109211

Kansas Certification IDs

9608 Loiret Boulevard, Lenexa, KS 66219

A2L_A Certification \#: 2456.01

Arkansas Certification \#: 05-008-0

Illinois Certification \#: 001191

lowa Certification \#: 118

Kansas/NELAP Certification \#: E-10116
Louisiana Certification \#: 03055

Nevada Certification \#: KSO00212008A

Oklahoma Certification \#: 9205/9935

Texas Certification \#: T104704407-08-TX

Utah Certification \#: 9135995665 


\section{SAMPLE SUMMARY}

$\begin{array}{ll}\text { Project: } & \text { KS/MO Waste Water } \\ \text { Pace Project No.: } & 60109211\end{array}$

\begin{tabular}{|c|c|c|c|c|}
\hline Lab ID & Sample ID & Matrix & Date Collected & Date Received \\
\hline 60109211001 & AGPURGE-W-10111 & Water & $10 / 31 / 1109: 00$ & $11 / 01 / 1109: 20$ \\
\hline 60109211002 & BAPURGE-W-10112 & Water & $10 / 31 / 1112: 55$ & 11/01/11 09:20 \\
\hline 60109211003 & CNPURGE-W-10113 & Water & 10/31/11 14:02 & $11 / 01 / 1109: 20$ \\
\hline 60109211004 & EUPURGE-W-10114 & Water & $10 / 31 / 11 \quad 15: 52$ & $11 / 01 / 1109: 20$ \\
\hline 60109211005 & HAPURGE-W-10115 & Water & $10 / 31 / 1112: 27$ & $11 / 01 / 1109: 20$ \\
\hline 60109211006 & MRPURGE-W-10116 & Water & $10 / 31 / 1114: 42$ & $11 / 01 / 1109: 20$ \\
\hline 60109211007 & SVPURGE-W-10117 & Water & $10 / 31 / 1118: 30$ & $11 / 01 / 1109: 20$ \\
\hline
\end{tabular}




\title{
SAMPLE ANALYTE COUNT
}

\begin{abstract}
Project: $\quad$ KS/MO Waste Water
Pace Project No:: 60109211
\end{abstract}

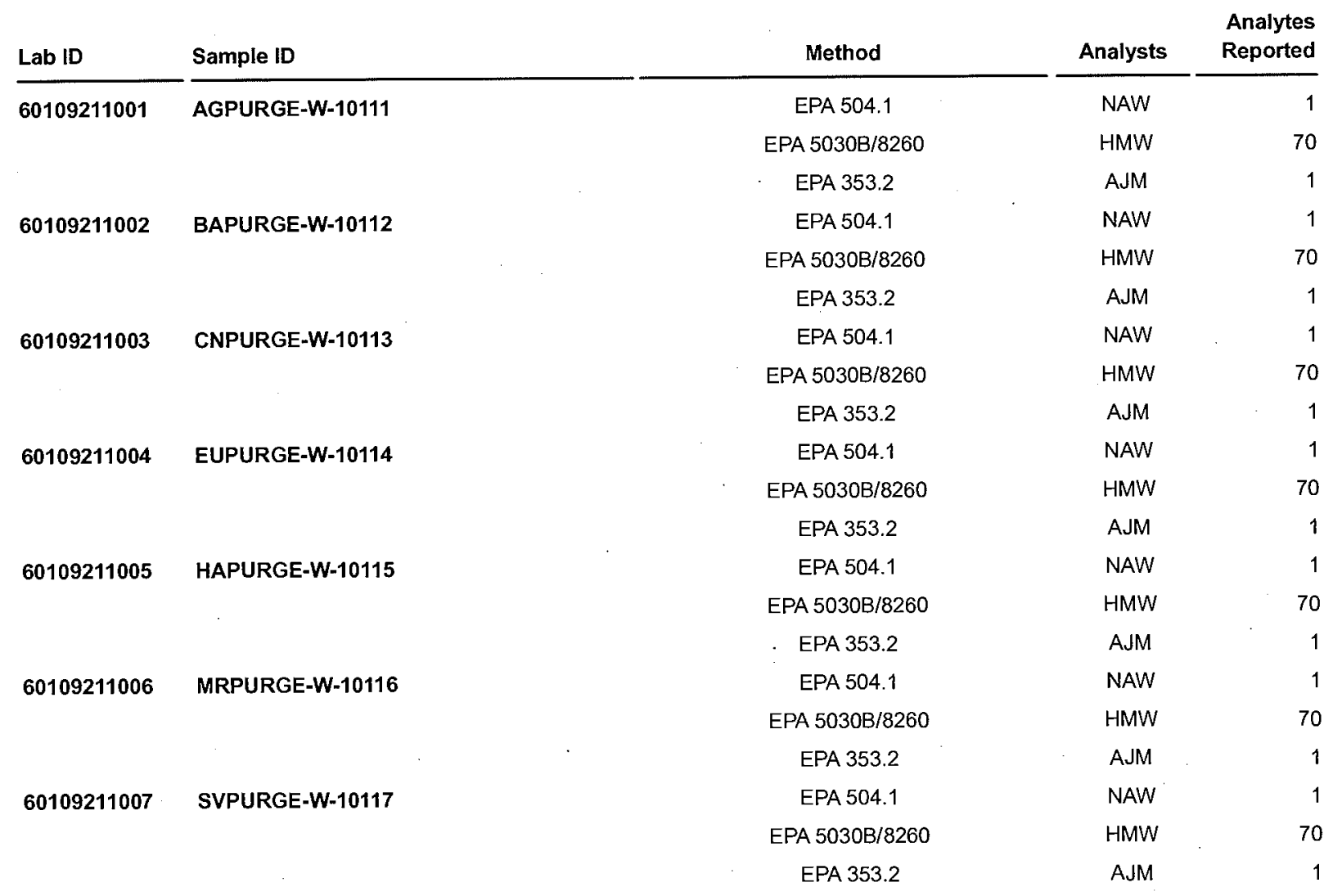




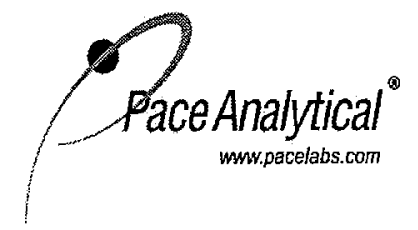

Pace Analytical Services, Inc. 9608 Loiret Blvd. Lenexa, KS 66219

(913)599-5665

\section{ANALYTICAL RESULTS}

Project: KS/MO Waste Water

Pace Project No.: 60109211

Sample: AGPURGE-W-10111 Lab ID: 60109211001 Collected: 10/31/11 09:00 Received: 11/01/11 09:20 Matrix: Water

\begin{tabular}{|c|c|c|c|c|c|c|c|}
\hline Parameters & Results & Units & Report Limit & DF & Prepared & Analyzed & CAS No. \\
\hline
\end{tabular}

504 GCS EDB and DBCP

1,2-Dibromoethane (EDB)

\section{MSV}

\section{Acetone}

Benzene

Bromobenzene

Bromochloromethane

Bromodichloromethane

\section{Bromoform}

Bromomethane

2-Butanone (MEK)

n-Butylbenzene

sec-Butylbenzene

tert-Butylbenzene

Carbon disulfide

Carbon tetrachloride

Chlorobenzene

Chloroethane

Chloroform

Chloromethane

2-Chlorotoluene

4-Chlorotoluene

1,2-Dibromo-3-chloropropane

Dibromochloromethane

1,2-Dibromoethane (EDB)

Dibromomethane

1,2-Dichlorobenzene

1,3-Dichlorobenzene

1,4-Dichlorobenzene

Dichlorodifluoromethane

1,1-Dichloroethane

1,2-Dichloroethane

1,2-Dichloroethene (Total)

1,1-Dichloroethene

cis-1,2-Dichloroethene

trans-1,2-Dichloroethene

1,2-Dichloropropane

1,3-Dichloropropane

2,2-Dichloropropane

1,1-Dichloropropene

cis-1,3-Dichloropropene

trans-1,3-Dichloropropene

Ethylbenzene

Hexachioro-1,3-butadiene

2-Hexanone

Isopropylbenzene (Cumene)

p-Isopropyltoluene

Date: 11/11/2011 11:15 AM
Analytical Method: EPA 504.1 Preparation Method: EPA 504.1

$$
\text { ND ug/L }
$$

$0.028111 / 07 / 1100: 00 \quad 11 / 08 / 11 \quad 00: 52 \quad 106-93-4$

Analytical Method: EPA 5030B/8260

\begin{tabular}{|c|c|c|c|c|}
\hline ND ug/L & 10.0 & 1 & $11 / 04 / 1120: 18$ & $67-64-1$ \\
\hline ND ug/L & 1.0 & 1 & $11 / 04 / 1120: 18$ & $71-43-2$ \\
\hline ND ug/L & 1.0 & 1 & $11 / 04 / 1120: 18$ & $108-86-1$ \\
\hline ND ug/L & 1.0 & 1 & $11 / 04 / 1120: 18$ & $74-97-5$ \\
\hline ND ug/L & 1.0 & 1 & $11 / 04 / 1120: 18$ & $75-27-4$ \\
\hline ND ug/L & 1.0 & 1 & $11 / 04 / 1120: 18$ & $75-25-2$ \\
\hline ND ug/L & 1.0 & 1 & $11 / 04 / 1120: 18$ & $74-83-9$ \\
\hline ND ug/L & 10.0 & 1 & $11 / 04 / 1120: 18$ & $78-93-3$ \\
\hline ND ug/L & 1.0 & 1 & $11 / 04 / 1120: 18$ & $104-51-8$ \\
\hline ND ug/L & 1.0 & 1 & $11 / 04 / 1120: 18$ & $135-98-8$ \\
\hline ND ug/L & 1.0 & 1 & $11 / 04 / 1120: 18$ & $98-06-6$ \\
\hline ND ug/L & 5.0 & 1 & $11 / 04 / 1120: 18$ & $75-15-0$ \\
\hline $21.8 \mathrm{ug} / \mathrm{L}$ & 1.0 & 1 & $11 / 04 / 1120: 18$ & $56-23-5$ \\
\hline ND ug/L & 1.0 & 1 & $11 / 04 / 1120: 18$ & $108-90-7$ \\
\hline ND ug/L & 1.0 & 1 & $11 / 04 / 1120: 18$ & $75-00-3$ \\
\hline $1.6 \mathrm{ug} / \mathrm{L}$ & 1.0 & 1 & $11 / 04 / 1120: 18$ & $67-66-3$ \\
\hline ND ug/L. & 1.0 & 1 & $11 / 04 / 1120: 18$ & $74-87-3$ \\
\hline ND ug/L & 1.0 & 1 & $11 / 04 / 1120: 18$ & $95-49-8$ \\
\hline ND ug/L & 1.0 & 1 & $11 / 04 / 1120: 18$ & $106-43-4$ \\
\hline ND ug/L & 2.5 & 1 & $11 / 04 / 1120: 18$ & $96-12-8$ \\
\hline ND ug/L & 1.0 & 1 & $11 / 04 / 1120: 18$ & $124-48-1$ \\
\hline ND ug/L & 1.0 & 1 & $11 / 04 / 1120: 18$ & $106-93-4$ \\
\hline ND ug/L & 1.0 & 1 & $11 / 04 / 1120: 18$ & $74-95-3$ \\
\hline ND ug/L & 1.0 & 1 & $11 / 04 / 1120: 18$ & $95-50-1$ \\
\hline ND ug/L & 1.0 & 1 & $11 / 04 / 1120: 18$ & $541-73-1$ \\
\hline ND ug/L & 1.0 & 1 & $11 / 04 / 1120: 18$ & $106-46-7$ \\
\hline ND ug/L & 1.0 & 1 & $11 / 04 / 1120: 18$ & $75-71-8$ \\
\hline ND ug/L & 1.0 & 1 & $11 / 04 / 1120: 18$ & $75-34-3$ \\
\hline ND ug/L & 1.0 & 1 & $11 / 04 / 1120: 18$ & $107-06-2$ \\
\hline ND ug/L & 1.0 & 1 & $11 / 04 / 1120: 18$ & $540-59-0$ \\
\hline ND ug/L & 1.0 & 1 & $11 / 04 / 1120: 18$ & $75-35-4$ \\
\hline ND ug/L & 1.0 & 1 & $11 / 04 / 1120: 18$ & $156-59-2$ \\
\hline ND ug/L & 1.0 & 1 & $11 / 04 / 1120: 18$ & $156-60-5$ \\
\hline ND ug/L & 1.0 & 1 & $11 / 04 / 1120: 18$ & $78-87-5$ \\
\hline ND ug/L & 1.0 & 1 & $11 / 04 / 1120: 18$ & $142-28-9$ \\
\hline ND ug/L & 1.0 & 1 & $11 / 04 / 1120: 18$ & $594-20-7$ \\
\hline ND ug/L & 1.0 & 1 & $11 / 04 / 1120: 18$ & $563-58-6$ \\
\hline ND ug/L & 1.0 & 1 & $11 / 04 / 1120: 18$ & $10061-01-5$ \\
\hline ND ug/L & 1.0 & 1 & $11 / 04 / 1120: 18$ & $10061-02-6$ \\
\hline ND ug/L & 1.0 & 1 & $11 / 04 / 1120: 18$ & $100-41-4$ \\
\hline ND ug/L & 1.0 & 1 & $11 / 04 / 1120: 18$ & $87-68-3$ \\
\hline ND ug/L & 10.0 & 1 & $11 / 04 / 1120: 18$ & $591-78-6$ \\
\hline ND ug/L & 1.0 & 1 & $11 / 04 / 1120: 18$ & $98-82-8$ \\
\hline ND ug/L & 1.0 & 1 & $11 / 04 / 1120: 18$ & $99-87-6$ \\
\hline
\end{tabular}

\section{REPORT OF LABORATORY ANALYSIS}

Page 5 of 26
This report shall not be reproduced, except in full, without the written consent of Pace Analytical Services, Inc.. 


\section{ANALYTICAL RESULTS}

Project: $\quad$ KS/MO Waste Water

Pace Project No: $\quad 60109211$

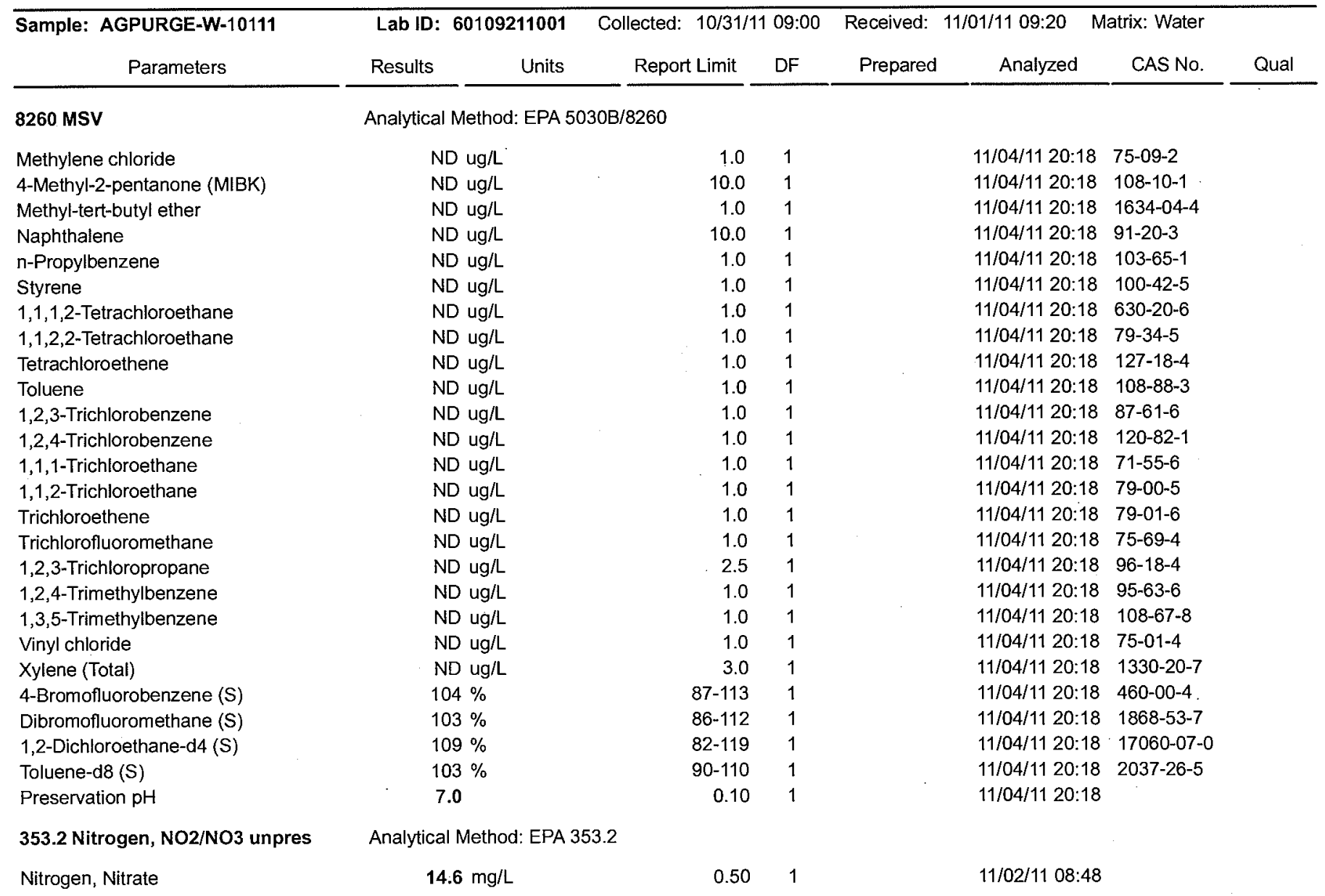




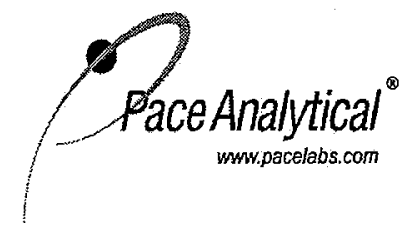

Pace Analytical Services, Inc. 9608 Loiret Blvd. Lenexa, KS 66219 (913)599-5665

\section{ANALYTICAL RESULTS}

Project: $\quad$ KS/MO Waste Water

Pace Project No.: 60109211

Sample: BAPURGE-W-10112 Lab ID: 60109211002 Collected: 10/31/11 12:55 Received: 11/01/11 09:20 Matrix: Water

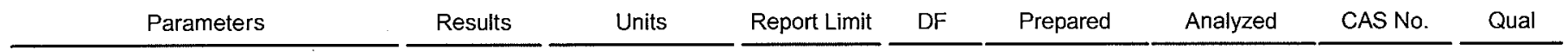

504 GCS EDB and DBCP

1,2-Dibromoethane (EDB)

\section{MSV}

\section{Acetone}

Benzene

Bromobenzene

Bromochloromethane

Bromodichloromethane

Bromoform

Bromomethane

2-Butanone (MEK)

n-Butylbenzene

sec-Butylbenzene

tert-Butylbenzene

Carbon disuifide

Carbọn tetrachloride

Chlorobenzene

Chloroethane

Chloroform

Chloromethane

2-Chlorotoluene

4-Chlorotoluene

1,2-Dibromo-3-chloropropane

Dibromochloromethane

1,2-Dibromoethane (EDB)

Dibromomethane

1,2-Dichlorobenzene

1,3-Dichlorobenzene

1,4-Dichlorobenzene

Dichlorodifluoromethane

1,1-Dichloroethane

1,2-Dichloroethane

1,2-Dichloroethene (Total)

1,1-Dichloroethene

cis-1,2-Dichloroethene

trans-1,2-Dichloroethene

1,2-Dichloropropane

1,3-Dichloropropane

2,2-Dichloropropane

1,1-Dichloropropene

cis-1,3-Dichloropropene

trans-1,3-Dichloropropene

Ethylbenzene

Hexachloro-1,3-butadiene

2-Hexanone

Isopropylbenzene (Cumene)

p-Isopropyltoluene

Date: 11/11/2011 11:15 AM

Analytical Method: EPA 504.1 Preparation Method: EPA 504.1

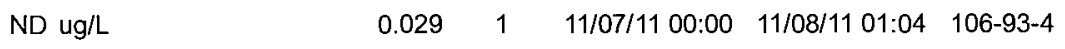

Analytical Method: EPA 5030B/8260

ND ug/L

ND ug/L

ND ug/L

ND ug/L

ND ug/L

ND ug/L

ND ug/L

ND ug/L

ND ug/L

ND ug/L

ND ug/L

ND ug/L

$1.1 \mathrm{ug} / \mathrm{L}$

ND ug/L

ND ug/L

ND ug/L

ND ug/L

$\mathrm{ND}$ ug/L

ND ug/L

ND ug/L

ND ug/L

ND ug/L

ND ug/L

ND ug/L

ND ug/L

ND ug/L

ND ug/L

ND ug/L

ND ug/L

ND ug/L

ND ug/L

ND ug/L

ND ug/L

ND ug/L

ND ug/L

ND ug/L

ND ug/L

ND ug/L

ND ug/L

ND ug/L

ND ug/L

ND ug/L

ND ug/L

ND ug/L

$\begin{array}{rr}10.0 & 1 \\ 1.0 & 1 \\ 1.0 & 1 \\ 1.0 & 1 \\ 1.0 & 1 \\ 1.0 & 1 \\ 1.0 & 1 \\ 10.0 & 1 \\ 1.0 & 1 \\ 1.0 & 1 \\ 1.0 & 1 \\ 5.0 & 1 \\ 1.0 & 1 \\ 1.0 & 1 \\ 1.0 & 1 \\ 1.0 & 1 \\ 1.0 & 1 \\ 1.0 & 1 \\ 1.0 & 1 \\ 2.5 & 1 \\ 1.0 & 1 \\ 1.0 & 1 \\ 1.0 & 1 \\ 1.0 & 1 \\ 1.0 & 1 \\ 1.0 & 1 \\ 1.0 & 1 \\ 1.0 & 1 \\ 1.0 & 1 \\ 1.0 & 1 \\ 1.0 & 1 \\ 1.0 & 1 \\ 1.0 & 1 \\ 1.0 & 1 \\ 1.0 & 1 \\ 1.0 & 1 \\ 1.0 & 1 \\ 1.0 & 1 \\ 1.0 & 1 \\ 1.0 & 1 \\ 1.0 & 1 \\ 10.0 & 1 \\ 1.0 & 1 \\ 1.0 & 1\end{array}$

11/04/11 20:35 67-64-1

11/04/11 20:35 71-43-2

$11 / 04 / 1120: 35$ 108-86-1

11/04/11 20:35 74-97-5

11/04/11 20:35 75-27-4

11/04/11 20:35 75-25-2

11/04/11 20:35 74-83-9

11/04/11 20:35 78-93-3

11/04/11 20:35 104-51-8

11/04/11 20:35 135-98-8

11/04/11 20:35 98-06-6

11/04/11 20:35 75-15-0

$11 / 04 / 1120: 35 \quad 56-23-5$

11/04/11 20:35 108-90-7

11/04/11 20:35 75-00-3

11/04/11 20:35 67-66-3

11/04/11 20:35 74-87-3

11/04/11 20:35 95-49-8

$11 / 04 / 1120: 35 \quad 106-43-4$

11/04/11 20:35 96-12-8

11/04/11 20:35 124-48-1

11/04/11 20:35 106-93-4

11/04/11 20:35 74-95-3

11/04/11 20:35 95-50-1

11/04/11 20:35 541-73-1

11/04/11 20:35 106-46-7

11/04/11 20:35 75-71-8

11/04/11 20:35 75-34-3

$11 / 04 / 1120: 35 \quad 107-06-2$

11/04/11 20:35 540-59-0

11/04/11 20:35 75-35-4

11/04/11 20:35 156-59-2

11/04/11 20:35 156-60-5

11/04/11 20:35 78-87-5

11/04/11 20:35 142-28-9

11/04/11 20:35 594-20-7

11/04/11 20:35 563-58-6

11/04/11 20:35 10061-01-5

11/04/11 20:35 10061-02-6

11/04/11 20:35 100-41-4

11/04/11 20:35 87-68-3

11/04/11 20:35 591-78-6

$11 / 04 / 1120: 35 \quad 98-82-8$

11/04/11 20:35 99-87-6

\section{REPORT OF LABORATORY ANALYSIS}

Page 7 of 26

This report shall, not be reproduced, except in full,

without the written consent of Pace Analytical Services, Inc.. 


\section{ANALYTICAL RESULTS}

Project: $\quad$ KS/MO Waste Water

Pace Project No.: 60109211

Sample: BAPURGE-W-10112

Lab ID: 60109211002 Collected: 10/31/11 12:55 Received: 11/01/11 09:20 Matrix: Water

\begin{tabular}{|c|c|c|c|c|c|}
\hline Parameters & Results & Report Limit & DF & Prepared & CAS No. \\
\hline 8260 MSV & Analytical Method: EPA 5 & 8260 & & & \\
\hline Methylene chloride & ND ug/L & 1.0 & 1 & $11 / 04 / 1120: 35$ & $75-09-2$ \\
\hline 4-Methyl-2-pentanone (MIBK) & ND ug/L & 10.0 & 1 & $11 / 04 / 1120: 35$ & $108-10-1$ \\
\hline Methyl-tert-butyl ether & ND ug/L & 1.0 & 1 & $11 / 04 / 1120: 35$ & $1634-04-4$ \\
\hline Naphthalene & ND ug/L & 10.0 & 1 & $11 / 04 / 1120: 35$ & $91-20-3$ \\
\hline n-Propylbenzene & ND ug/L & 1.0 & 1 & $11 / 04 / 1120: 35$ & $103-65-1$ \\
\hline Styrene & ND ug/L & 1.0 & 1 & $11 / 04 / 1120: 35$ & $100-42-5$ \\
\hline 1,1,1,2-Tetrachloroethane & ND ug/L & 1.0 & 1 & $11 / 04 / 1120: 35$ & $630-20-6$ \\
\hline 1,1,2,2-Tetrachloroethane & ND ug/L & 1.0 & 1 & $11 / 04 / 1120: 35$ & $79-34-5$ \\
\hline Tetrachloroethene & ND ug/L & 1.0 & 1 & $11 / 04 / 1120: 35$ & $127-18-4$ \\
\hline Toluene & ND ug/L & 1.0 & 1 & $11 / 04 / 1120: 35$ & $108-88-3$ \\
\hline 1,2,3-Trichlorobenzene & ND ug/L & 1.0 & 1 & $11 / 04 / 1120: 35$ & $87-61-6$ \\
\hline $1,2,4$-Trichlorobenzene & ND ug/L & 1.0 & 1 & $11 / 04 / 1120: 35$ & $120-82-1$ \\
\hline 1,1,1-Trichloroethane & ND ug/L & 1.0 & 1 & $11 / 04 / 1120: 35$ & $71-55-6$ \\
\hline 1,1,2-Trichloroethane & ND ug/L & 1.0 & 1 & $11 / 04 / 1120: 35$ & $79-00-5$ \\
\hline Trichloroethene & ND ug/L & 1.0 & 1 & $11 / 04 / 1120: 35$ & $79-01-6$ \\
\hline Trichlorofluoromethane & ND ug/L & 1.0 & 1 & $11 / 04 / 1120: 35$ & $75-69-4$ \\
\hline 1,2,3-Trichloropropane & ND ug/L & 2.5 & 1 & $11 / 04 / 1120: 35$ & $96-18-4$ \\
\hline $1,2,4$-Trimethylbenzene & ND ug/L & 1.0 & 1 & $11 / 04 / 1120: 35$ & $95-63-6$ \\
\hline $1,3,5$-Trimethylbenzene & ND ug/L & 1.0 & 1 & $11 / 04 / 1120: 35$ & $108-67-8$ \\
\hline Vinyl chloride & ND ug/L & 1.0 & 1 & $11 / 04 / 1120: 35$ & $75-01-4$ \\
\hline Xylene (Total) & ND ug/L & 3.0 & 1 & $11 / 04 / 1120: 35$ & $1330-20-7$ \\
\hline 4-Bromofluorobenzene (S) & $102 \%$ & $87-113$ & 1 & $11 / 04 / 1120: 35$ & $460-00-4$ \\
\hline Dibromofluoromethane (S) & $98 \%$ & $86-112$ & 1 & $11 / 04 / 1120: 35$ & $1868-53-7$ \\
\hline 1,2-Dichloroethane-d4 (S) & $101 \%$ & $82-119$ & 1 & $11 / 04 / 1120: 35$ & $17060-07-0$ \\
\hline Toluene-d8 (S) & $95 \%$ & $90-110$ & 1 & $11 / 04 / 1120: 35$ & $2037-26-5$ \\
\hline Preservation $\mathrm{pH}$ & 7.0 & 0.10 & 1 & $11 / 04 / 1120: 35$ & \\
\hline
\end{tabular}

353.2 Nitrogen, $\mathrm{NO} / \mathrm{NO} 3$ unpres

Nitrogen, Nitrate
Analytical Method: EPA 353.2

$6.1 \mathrm{mg} / \mathrm{L}$ 


\section{ANALYTICAL RESULTS}

Project: . KS/MO Waste Water

Pace Project No.: 60109211

Sample: CNPURGE-W-10113 Lab ID: 60109211003 $\quad$ Collected: 10/31/11 14:02 Received: 11/01/11 09:20 Matrix: Water

Parameters $\quad$ Results $\longrightarrow$ Units $\quad$ Report Limit DF Prepared Analyzed CAS No.

504 GCS EDB and DBCP

1,2-Dibromoethane (EDB)

8260 MSV

Acetone
Benzene
Bromobenzene
Bromochloromethane
Bromodichloromethane
Bromoform
Bromomethane
2-Butanone (MEK)
n-Butylbenzene
sec-Butylbenzene
tert-Butylbenzene
Carbon disulfide
Carbon tetrachloride
Chlorobenzene
Chloroethane
Chloroform
Chloromethane
2-Chlorotoluene
4-Chlorotoluene
1,2-Dibromo-3-chloropropane
Dibromochloromethane
1,2-Dibromoethane (EDB)
Dibromomethane
1,2-Dichlorobenzene
1,3-Dichlorobenzene
1,4-Dichlorobenzene
Dichlorodifluoromethane
1,1-Dichloroethane
1,2-Dichloroethane
1,2-Dichloroethene (Total)
1,1-Dichloroethene
cis-1,2-Dichloroethene
trans-1,2-Dichloroethene
1,2-Dichloropropane
1,3-Dichloropropane
2,2-Dichloropropane
1,1-Dichloropropene
cis-1,3-Dichloropropene
trans-1,3-Dichloropropene
Ethylbenzene
Hexachloro-1,3-butadiene
2-Hexanone
Isopropylbenzene (Cumene)
p-Isopropyltoluene
B.

Date: 11/11/2011 11:15 AM
Analytical Method: EPA 504.1 Preparation Method: EPA 504.1

$\begin{array}{lllllll}\text { ND ug/L } \quad 0.028 & 1 & 11 / 07 / 11 & 00: 00 & 11 / 08 / 11 & 01: 17 & 106-93-4\end{array}$

Analytical Method: EPA 5030B/8260

\begin{tabular}{|c|c|}
\hline ND ug/L & 10.0 \\
\hline ND ug/L & 1.0 \\
\hline ND ug/L & 1.0 \\
\hline ND ug/L & 1.0 \\
\hline ND ug/L & 1.0 \\
\hline ND ug/L & 1.0 \\
\hline ND ug/L & 1.0 \\
\hline ND ug/L & 10.0 \\
\hline$N D$ ug/L & 1.0 . \\
\hline$N D$ ug/L & 1.0 \\
\hline ND ug/L & 1.0 \\
\hline ND ug/L & 5.0 \\
\hline $1.3 \mathrm{ug} / \mathrm{L}$ & 1.0 \\
\hline ND ug/L & 1.0 \\
\hline ND ug/L & 1.0 \\
\hline ND ug/L & 1.0 \\
\hline ND ug/L & 1.0 \\
\hline ND ug/L & 1.0 \\
\hline ND ug/L & 1.0 \\
\hline ND ug/L & 2.5 \\
\hline ND ug/L & 1.0 \\
\hline ND ug/L & 1.0 \\
\hline ND ug/L & 1.0 \\
\hline ND ug/L & 1.0 \\
\hline ND ug/L & 1.0 \\
\hline ND ug/L & 1.0 \\
\hline ND ug/L & 1.0 \\
\hline ND ug/L & 1.0 \\
\hline ND ug/L & 1.0 \\
\hline ND ug/L & 1.0 \\
\hline ND ug/L & 1.0 \\
\hline ND ug/L & 1.0 \\
\hline ND ug/L & 1.0 \\
\hline ND ug/L & 1.0 \\
\hline ND ug/L & 1.0 \\
\hline ND ug/L & 1.0 \\
\hline ND ug/L & 1.0 \\
\hline ND ug/L & 1.0 \\
\hline ND ug/L & 1.0 \\
\hline ND ug/L & 1.0 \\
\hline ND ug/L & 1.0 \\
\hline ND ug/L & 10.0 \\
\hline ND ug/L & 1.0 \\
\hline ND ug/L & 1.0 \\
\hline
\end{tabular}

11/04/11 20:51 67-64-1 11/04/11 20:51 71-43-2

$11 / 04 / 1120: 51 \quad 108-86-1$

11/04/11 20:51 74-97-5

$11 / 04 / 1120: 51 \quad 75-27-4$

$11 / 04 / 1120: 51 \quad 75-25-2$

11/04/11 20:51 74-83-9

$11 / 04 / 1120: 51 \quad 78-93-3$

$11 / 04 / 1120: 51 \quad 104-51-8$

$11 / 04 / 1120: 51 \quad 135-98-8$

11/04/11 20:51 98-06-6

$11 / 04 / 1120: 51 \quad 75-15-0$

11/04/11 20:51 56-23-5

11/04/11 20:51 108-90-7

11/04/11 20:51 75-00-3

$11 / 04 / 1120: 51 \quad 67-66-3$

11/04/11 20:51 74-87-3

$11 / 04 / 1120: 51 \quad 95-49-8$

11/04/11 20:51 106-43-4

$11 / 04 / 1120: 51 \quad 96-12-8$

11/04/11 20:51 124-48-1

11/04/11 20:51 106-93-4

$11 / 04 / 1120: 51 \quad 74-95-3$

$11 / 04 / 1120: 51$ 95-50-1

11/04/11 20:51 541-73-1

$11 / 04 / 1120: 51 \quad 106-46-7$

$11 / 04 / 1120: 51 \quad 75-71-8$

$11 / 04 / 1120: 51 \quad 75-34-3$

$11 / 04 / 1120: 51 \quad 107-06-2$

11/04/11 20:51 540-59-0

$11 / 04 / 1120: 51 \quad 75-35-4$

$11 / 04 / 1120: 51 \quad 156-59-2$

11/04/11 20:51 156-60-5

$11 / 04 / 1120: 51 \quad 78-87-5$

11/04/11 20:51 142-28-9

11/04/11 20:51 594-20-7

11/04/11 20:51 563-58-6

11/04/11 20:51 10061-01-5

11/04/11 20:51 10061-02-6

11/04/11 20:51 100-41-4

11/04/11 20:51 87-68-3

11/04/11 20:51 591-78-6

$11 / 04 / 1120: 51 \quad 98-82-8$

11/04/11 20:51 99-87-6

\section{REPORT OF LABORATORY ANALYSIS}

Page 9 of 26

This report shall not be reproduced, except in full,

without the written consent of Pace Analytical Services, Inc.. 


\section{ANALYTICAL RESULTS}

Project: KS/MO Waste Water

Pace Project No.: 60109211

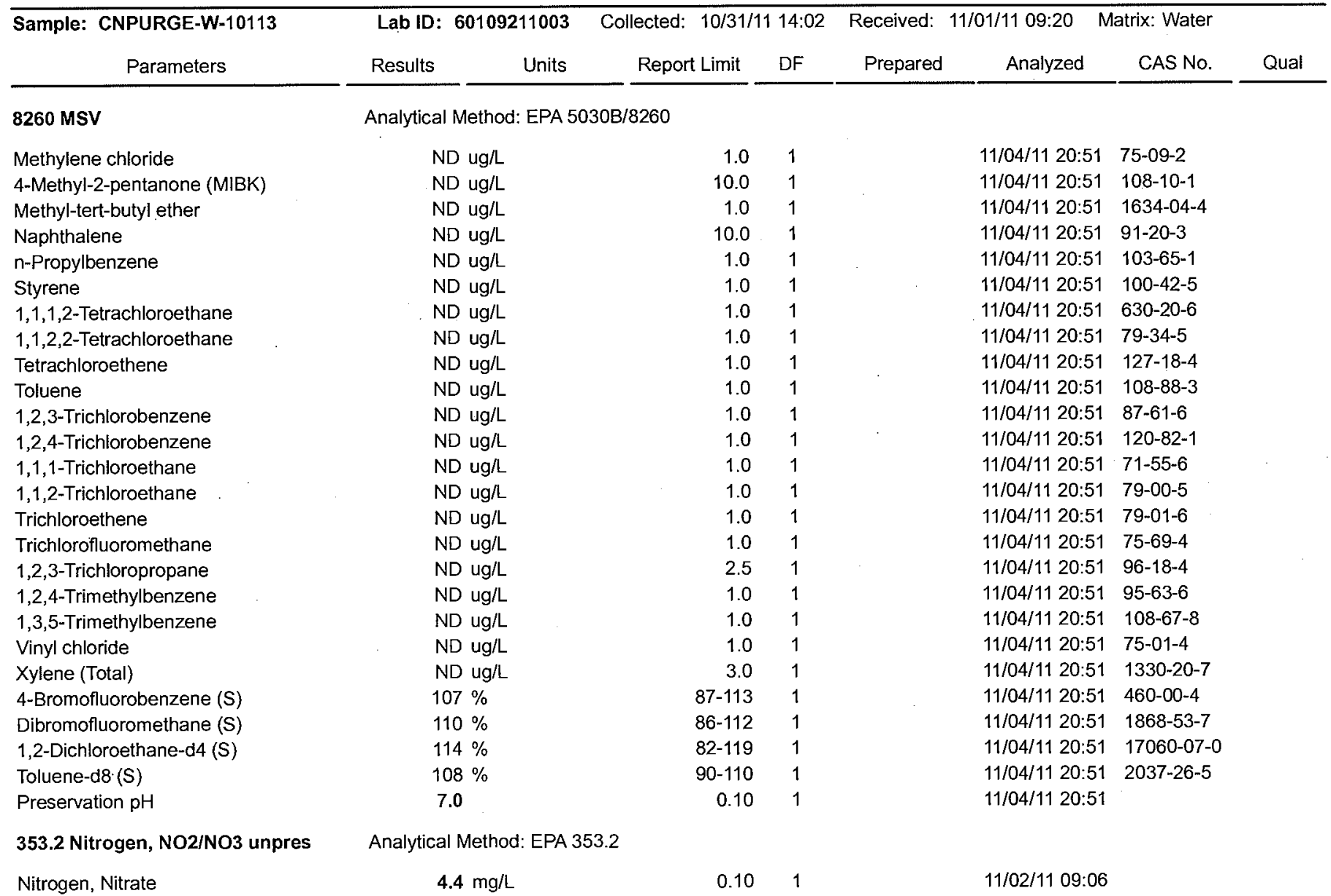




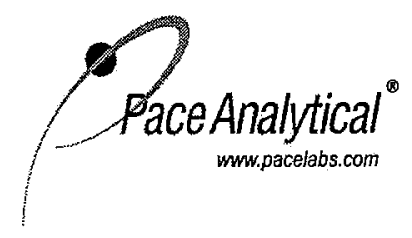

Pace Analytical Services, Inc. 9608 Loiret Blvo. Lenexa, KS 66219

(913)599-5665

\section{ANALYTICAL RESULTS}

Project: KS/MO Waste Water

Pace Project No.: 60109211

\begin{tabular}{|c|c|c|c|c|c|c|c|c|}
\hline Sample: EUPURGE-W-10114 & Lab ID: & 60109211004 & Collected: $10 / 31 /$ & $15: 52$ & Received: & $11 / 01 / 1109: 20$ & Matrix: Water & \\
\hline Parameters & Results & Units & Report Limit & DF & Prepared & Analyzed & CAS No. & Qual \\
\hline
\end{tabular}

504 GCS EDB and DBCP

1,2-Dibromoethane (EDB)

\section{MSV}

Acetone

Benzene

Bromobenzene

Bromochloromethane

Bromodichloromethane

Bromoform

Bromomethane

2-Butanone (MEK)

n-Butylbenzene

sec-Butylbenzene

tert-Butylbenzene

Carbon disulfide

Carbon tetrachloride

Chlorobenzene

Chloroethane

Chloroform

Chloromethane

2-Chlorotoluene

4-Chlorotoluene

1,2-Dibromo-3-chloropropane

Dibromochloromethane

1,2-Dibromoethane (EDB)

Dibromomethane

1,2-Dichlorobenzene

1,3-Dichlorobenzene

1,4-Dichlorobenzene

Dichlorodifluoromethane

1,1-Dichloroethane

1,2-Dichloroethane

1,2-Dichloroethene (Total)

1,1-Dichloroethene

cis-1,2-Dichloroethene

trans-1,2-Dichloroethene

1,2-Dichloropropane

1,3-Dichloropropane

2,2-Dichloropropane

1,1-Dichloropropene cis-1,3-Dichloropropene

trans-1,3-Dichloropropene

Ethylbenzene

Hexachloro-1,3-butadiene

2-Hexanone

Isopropylbenzene (Cumene)

p-Isopropyltoluene

Date: 11/11/2011 11:15 AM
Analytical Method: EPA 504.1 Preparation Method: EPA 504.1

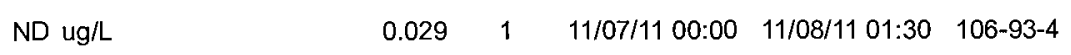

Analytical Method: EPA 5030B/8260

\begin{tabular}{|c|c|c|c|c|}
\hline$N D$ ug/L & 10.0 & 1 & $11 / 04 / 1121: 07$ & $67-64-1$ \\
\hline ND ug/L & 1.0 & 1 & $11 / 04 / 1121: 07$ & $71-43-2$ \\
\hline$N D u g / L$ & 1.0 & 1 & $11 / 04 / 1121: 07$ & $108-86-1$ \\
\hline ND ug/L & 1.0 & 1 & $11 / 04 / 1121: 07$ & $74-97-5$ \\
\hline ND ug/L & 1.0 & 1 & $11 / 04 / 1121: 07$ & $75-27-4$ \\
\hline ND ug/L & 1.0 & 1 & $11 / 04 / 1121: 07$ & $75-25-2$ \\
\hline ND ug/L & 1.0 & 1 & $11 / 04 / 1121: 07$ & $74-83-9$ \\
\hline ND ug/L & 10.0 & 1 & $11 / 04 / 1121: 07$ & $78-93-3$ \\
\hline ND ug/h & 1.0 & 1 & $11 / 04 / 1121: 07$ & $104-51-8$ \\
\hline ND ug/L & 1.0 & 1 & $11 / 04 / 1121: 07$ & $135-98-8$ \\
\hline ND ug/L & 1.0 & 1 & $11 / 04 / 1121: 07$ & $98-06-6$ \\
\hline ND ug/L & 5.0 & 1 & $11 / 04 / 1121: 07$ & $75-15-0$ \\
\hline ND ug/L & 1.0 & 1 & $11 / 04 / 1121: 07$ & $56-23-5$ \\
\hline ND ug/L & 1.0 & 1 & $11 / 04 / 1121: 07$ & $108-90-7$ \\
\hline ND ug/L & 1.0 & 1 & $11 / 04 / 1121: 07$ & $75-00-3$ \\
\hline ND ug/L & 1.0 & 1 & $11 / 04 / 1121: 07$ & $67-66-3$ \\
\hline ND ug/L & 1.0 & 1 & $11 / 04 / 1121: 07$ & $74-87-3$ \\
\hline ND ug/L & 1.0 & 1 & $11 / 04 / 1121: 07$ & $95-49-8$ \\
\hline ND ug/L & 1.0 & 1 & $11 / 04 / 1121: 07$ & $106-43-4$ \\
\hline ND ug/L & 2.5 & 1 & $11 / 04 / 1121: 07$ & $96-12-8$ \\
\hline ND ug/L & 1.0 & 1 & $11 / 04 / 1121: 07$ & $124-48-1$ \\
\hline ND ug/L & 1.0 & 1 & $11 / 04 / 1121: 07$ & $106-93 \cdot 4$ \\
\hline ND ug/L & 1.0 & 1 & $11 / 04 / 1121: 07$ & $74-95-3$ \\
\hline ND ug/L & 1.0 & 1 & $11 / 04 / 1121: 07$ & $95-50-1$ \\
\hline ND ug/L & 1.0 & 1 & $11 / 04 / 1121: 07$ & $541-73-1$ \\
\hline ND ug/L & 1.0 & 1 & $11 / 04 / 1121: 07$ & $106-46-7$ \\
\hline ND ug/L & 1.0 & 1 & $11 / 04 / 1121: 07$ & $75-71-8$ \\
\hline ND ug/L & 1.0 & 1 & $11 / 04 / 1121: 07$ & $75-34-3$ \\
\hline ND ug/L & 1.0 & 1 & $11 / 04 / 1121: 07$ & $107-06-2$ \\
\hline ND ug/L & 1.0 & 1 & $11 / 04 / 1121: 07$ & $540-59-0$ \\
\hline ND ug/L & 1.0 & 1 & $11 / 04 / 1121: 07$ & $75-35-4$ \\
\hline ND ug/L & 1.0 & 1 & $11 / 04 / 1121: 07$ & $156-59-2$ \\
\hline ND ug/L & 1.0 & 1 & $11 / 04 / 1121: 07$ & $156-60-5$ \\
\hline ND ug/L & 1.0 & 1 & $11 / 04 / 1121: 07$ & $78-87-5$ \\
\hline ND ug/L & 1.0 & 1 & $11 / 04 / 1121: 07$ & $142-28-9$ \\
\hline ND ug/L & 1.0 & 1 & $11 / 04 / 1121: 07$ & $594-20-7$ \\
\hline ND ug/L & 1.0 & 1 & $11 / 04 / 1121: 07$ & $563-58-6$ \\
\hline ND ug/L & 1.0 & 1 & $11 / 04 / 1121: 07$ & $10061-01-5$ \\
\hline ND ug/h & 1.0 & 1 & $11 / 04 / 1121: 07$ & $10061-02-6$ \\
\hline ND ug/L & 1.0 & 1 & $11 / 04 / 1121: 07$ & $100-41-4$ \\
\hline ND ug/L & 1.0 & 1 & $11 / 04 / 1121: 07$ & $87-68-3$ \\
\hline ND ug/L & 10.0 & 1 & $11 / 04 / 1121: 07$ & $591-78-6$ \\
\hline ND ug/L & 1.0 & 1 & $11 / 04 / 1121: 07$ & $98-82-8$ \\
\hline ND ug/L & 1.0 & 1 & $11 / 04 / 1121: 07$ & $99-87-6$ \\
\hline
\end{tabular}

REPORT OF LABORATORY ANALYSIS

Page 11 of 26
This report shall not be reproduced, except in full, without the written consent of Pace Analytical Services, Inc.. 


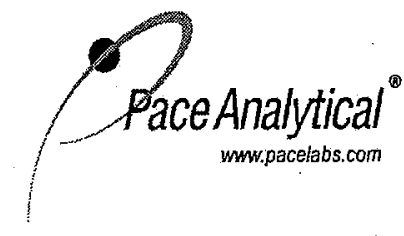

Pace Analytical Services, Inc. 9608 Loiret Blvd. Lenexa, KS 66219 (913)599-5665

\section{ANALYTICAL RESULTS}

Project: $\quad$ KS/MO Waste Water

Pace Project No.: 60109211

Sample: EUPURGE-W-10114 Lab ID: $60109211004 \quad$ Collected: 10/31/11 15:52 Received: 11/01/11 09:20 Matrix: Water

Parameters - Results $\longrightarrow$ Units - Report Limit DF Prepared

\section{MSV}

Methylene chloride 4-Methyl-2-pentanone (MIBK) Methyl-tert-butyl ether Naphthalene n-Propylbenzene Styrene

$1,1,1,2-$ Tetrachloroethane 1,1,2,2-Tetrachloroethane Tetrachloroethene

$$
\text { Toluene }
$$

1,2,3-Trichlorobenzene

1,2,4-Trichlorobenzene

1,1,1-Trichloroethane

1,1,2-Trichloroethane

Trichloroethene

Trichlorofluoromethane

1,2,3-Trichloropropane

1,2,4-Trimethylbenzene

1,3,5-Trimethylbenzene

Vinyl chloride

Xylene (Total)

4-Bromofluorobenzene (S)

Dibromofluoromethane (S)

1,2-Dichloroethane-d4 (S)

Toluene-d8 (S)

Preservation $\mathrm{pH}$

353.2 Nitrogen, NO2/NO3 unpres

Nitrogen, Nitrate

Analytical Method: EPA 5030B/8260

\begin{abstract}
ND ug/L
ND ug/L

ND ug/L

ND ug/L

ND ug/L

ND ug/L

ND ug/L

ND ug/L

$N D$ ug/L

ND ug/L

ND ug/L

ND ug/L

ND ug/L

ND ug/L

ND ug/L

ND ug/L

ND ug/L

ND ug/L

ND ug/L

ND ug/L

ND ug/L

$106 \%$

$108 \%$

$113 \%$

$105 \%$

7.0
\end{abstract}

Analytical Method: EPA 353.2

$10.6 \mathrm{mg} / \mathrm{L}$

$\begin{array}{rr}1.0 & 1 \\ 10.0 & 1 \\ 1.0 & 1 \\ 10.0 & 1 \\ 1.0 & 1 \\ 1.0 & 1 \\ 1.0 & 1 \\ 1.0 & 1 \\ 1.0 & 1 \\ 1.0 & 1 \\ 1.0 & 1 \\ 1.0 & 1 \\ 1.0 & 1 \\ 1.0 & 1 \\ 1.0 & 1 \\ 1.0 & 1 \\ 2.5 & 1 \\ 1.0 & 1 \\ 1.0 & 1 \\ 1.0 & 1 \\ 3.0 & 1 \\ 87-113 & 1 \\ 86-112 & 1 \\ 82-119 & 1 \\ 90-110 & 1 \\ 0.10 & 1\end{array}$

$0.50 \quad 1$
11/04/11 21:07 75-09-2

11/04/11 21:07 108-10-1

11/04/11 21:07 1634-04-4

11/04/11 21:07 91-20-3

11/04/11 21:07 103-65-1

$11 / 04 / 1121: 07 \quad 100-42-5$

11/04/11 21:07 630-20-6

11/04/11 21:07 79-34-5

11/04/11 21:07 127-18-4

11/04/11 21:07 108-88-3

11/04/11 21:07 87-61-6

11/04/11 21:07 120-82-1

11/04/11 21:07 71-55-6

11/04/11 21:07 79-00-5

11/04/11 21:07 79-01-6

11/04/11 21:07 75-69-4

11/04/11 21:07 96-18-4

11/04/11 21:07 95-63-6

11/04/11 21:07 108-67-8

11/04/11 21:07 75-01-4

11/04/11 21:07 1330-20-7

11/04/11 21:07 460-00-4

11/04/11 21:07 1868-53-7

11/04/11 21:07 17060-07-0

11/04/11 21:07 2037-26-5

11/04/11 21:07

$11 / 02 / 1109: 21$ 


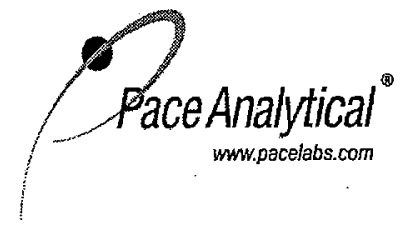

Pace Analytical Services, Inc. 9608 Loiret Blvd.

Lenexa, KS 66219

(913)599-5665

\section{ANALYTICAL RESULTS}

Project: $\quad$ KS/MO Waste Water

Pace Project No: 60109211

\begin{tabular}{|c|c|c|c|c|c|c|c|c|}
\hline Sample: HAPURGE-W-10115 & Lab ID: & 60109211005 & Collected: $10 / 31 / 1$ & $12: 27$ & Received: & $11 / 01 / 1109: 20$ & Matrix: Water & \\
\hline Parameters & Results & Units & Report Limit & DF & Prepared & Analyzed & CAS No. & Qual \\
\hline
\end{tabular}

504 GCS EDB and DBCP

1,2-Dibromoethane (EDB)

\section{MSV}

Acetone

Benzene

Bromobenzene

Bromochloromethane

Bromodichloromethane

Bromoform

Bromomethane

2-Butanone (MEK)

n-Butylbenzene

sec-Butylbenzene

tert-Butylbenzene

Carbon disulfide

Carbon tetrachloride

Chlorobenzene

Chloroethane

Chloroform

Chloromethane

2-Chlorotoluene

4-Chlorotoluene

1,2-Dibromo-3-chloropropane

Dibromochloromethane

1,2-Dibromoethane (EDB)

Dibromomethane

1,2-Dichlorobenzene

1,3-Dichlorobenzene

1,4-Dichlorobenzene

Dichlorodifluoromethane

1,1-Dichloroethane

1,2-Dichloroethane

1,2-Dichloroethene (Total)

1,1-Dichloroethene

cis-1,2-Dichloroethene

trans-1,2-Dichloroethene

1,2-Dichloropropane

1,3-Dichloropropane

2,2-Dichloropropane

1,1-Dichloropropene

cis-1,3-Dichloropropene

trans-1,3-Dichloropropene

Ethylbenzene

Hexachloro-1,3-butadiene

2-Hexanone

Isopropylbenzene (Cumene)

p-Isopropyitoluene

Date: 11/11/2011 11:15 AM

Analytical Method: EPA 504.1 Preparation Method: EPA 504.1

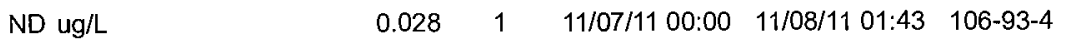

Analytical Method: EPA 5030B/8260

ND ug/L

ND ug/L

ND ug/L

ND ug/L

ND ug/L

ND ug/L

ND ug/L

ND ug/L

ND $u g / L$

ND ug/L

ND ug/L

ND ug/L

$6.1 \mathrm{ug} / \mathrm{L}$

ND ug/L

ND ug/L

ND ug/L

ND ug/L

ND ug/L

ND ug/L

ND ug/L

ND ug/L

ND ug/L

ND ug/L

ND ug/L

ND ug/L

ND ug/L

ND ug/L

ND ug/L

ND ug/L

ND ug/L

ND ug/L

ND ug/L

ND ug/L

ND ug/L

ND ug/L

ND ug/L

ND ug/L

ND ug/L

ND ug/L

ND ug/L

ND ug/L

ND ug/L

ND ug/L

ND ug/L

$\begin{array}{rr}10.0 & 1 \\ 1.0 & 1 \\ 1.0 & 1 \\ 1.0 & 1 \\ 1.0 & 1 \\ 1.0 & 1 \\ 1.0 & 1 \\ 10.0 & 1 \\ 1.0 & 1 \\ 1.0 & 1 \\ 1.0 & 1 \\ 5.0 & 1 \\ 1.0 & 1 \\ 1.0 & 1 \\ 1.0 & 1 \\ 1.0 & 1 \\ 1.0 & 1 \\ 1.0 & 1 \\ 1.0 & 1 \\ 1 \\ 10.5 & 1 \\ 1.0 & 1 \\ 1.0 & 1 \\ 1.0 & 1 \\ 1.0 & 1 \\ 1.0 & 1 \\ 1.0 & 1 \\ 1.0 & 1 \\ 1.0 & 1 \\ 1.0 & 1 \\ 1.0 & 1 \\ 1.0 & 1 \\ 1.0 & 1 \\ 1.0 & 1 \\ 1.0 & 1 \\ 1.0 & 1 \\ 1.0 & 1 \\ 1.0 & 1 \\ 1.0 & 1 \\ 1.0 & 1 \\ 1.0 & 1 \\ 1 \\ 1.0\end{array}$

11/04/11 21:24 67-64-1

$11 / 04 / 1121: 24 \quad 71-43-2$

11/04/11 21:24 108-86-1

11/04/11 21:24 74-97-5

11/04/11 21:24 75-27-4

$11 / 04 / 1121: 24 \quad 75-25-2$

$11 / 04 / 1121: 24 \quad 74-83-9$

11/04/11 21:24 78-93-3

$11 / 04 / 1121: 24 \quad 104-51-8$

$11 / 04 / 1121: 24 \quad 135-98-8$

11/04/11 21:24 98-06-6

11/04/11 21:24 75-15-0

$11 / 04 / 1121: 24 \quad 56-23-5$

11/04/11 21:24 108-90-7

$11 / 04 / 1121: 24 \quad 75-00-3$

$11 / 04 / 1121: 24 \quad 67-66-3$

11/04/11 21:24 74-87-3

11/04/11 21:24 95-49-8

$11 / 04 / 1121: 24$ 106-43-4

$11 / 04 / 1121: 24 \quad 96-12-8$

$11 / 04 / 1121: 24 \quad 124-48-1$

$11 / 04 / 1121: 24 \quad 106-93-4$

$11 / 04 / 1121: 24 \quad 74-95-3$

11/04/11 21:24 95-50-1

$11 / 04 / 1121: 24 \quad 541-73-1$

$11 / 04 / 1121: 24$ 106-46-7

11/04/11 21:24 75-71-8

11/04/11 21:24 75-34-3

11/04/11 21:24 107-06-2

11/04/11 21:24 540-59-0

11/04/11 21:24 75-35-4

$11 / 04 / 1121: 24 \quad 156-59-2$

$11 / 04 / 1121: 24 \quad 156-60-5$

11/04/11 21:24 78-87-5

11/04/11 21:24 142-28-9

11/04/11 21:24 594-20-7

$11 / 04 / 1121: 24 \quad 563-58-6$

$11 / 04 / 1121: 24 \quad 10061-01-5$

11/04/11 21:24 10061-02-6

$11 / 04 / 1121: 24 \quad 100-41-4$

$11 / 04 / 1121: 24 \quad 87-68-3$

11/04/11 21:24 591-78-6

$11 / 04 / 1121: 24 \quad 98-82-8$

11/04/11 21:24 99-87-6

REPORT OF LABORATORY ANALYSIS

This report shall not be reproduced, except in full,

without the written consent of Pace Analytical Services, Inc.. 


\section{ANALYTICAL RESULTS}

Project: KS/MO Waste Water

Pace Project No.: 60109211

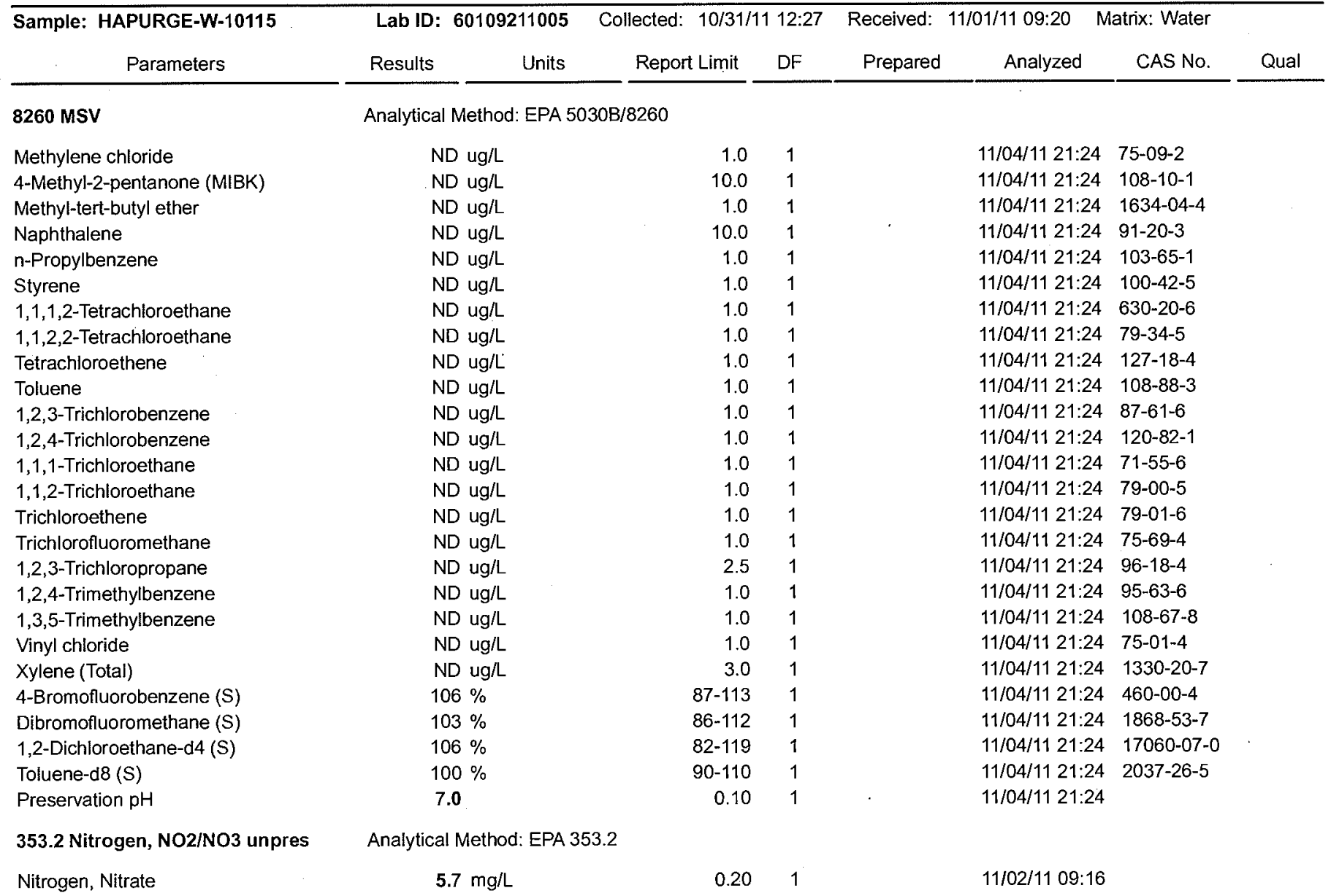




\section{ANALYTICAL RESULTS}

Project: $\quad$ KS/MO Waste Water

Pace Project No.: 60109211

\begin{tabular}{|c|c|c|c|c|c|c|c|c|}
\hline Sample: MRPURGE-W-10116 & Lab ID: & 60109211006 & Collected: $10 / 31 /$ & $14: 42$ & Received: & 11/01/11 09:20 & Matrix: Water & \\
\hline Parameters & Results & Units & Report Limit & $\mathrm{DF}$ & Prepared & Analyzed & CAS No. & Qual \\
\hline
\end{tabular}

504 GCS EDB and DBCP

1,2-Dibromoethane (EDB)

\section{$8260 \mathrm{MSV}$}

Acetone

Benzene

Bromobenzene

Bromochloromethane

Bromodichloromethane

Bromoform

Bromomethane

2-Butanone (MEK)

n-Butylbenzene

sec-Butylbenzene

tert-Butylbenzene

Carbon disulfide

Carbon tetrachloride

Chlorobenzene

Chloroethane

Chloroform

Chloromethane

2-Chlorotoluene

4-Chlorotoluene

1,2-Dibromo-3-chloropropane

Dibromochloromethane

1,2-Dibromoethane (EDB)

Dibromomethane

1,2-Dichlorobenzene

1,3-Dichlorobenzene

1,4-Dichlorobenzene

Dichlorodifluoromethane

1,1-Dichloroethane

1,2-Dichloroethane

1,2-Dichloroethene (Total)

1,1-Dichloroethene

cis-1,2-Dichloroethene

trans-1,2-Dichloroethene

1,2-Dichloropropane

1,3-Dichloropropane

2,2-Dichloropropane

1,1-Dichloropropene

cis-1,3-Dichloropropene

trans-1,3-Dichloropropene

Ethylbenzene

Hexachloro-1,3-butadiene

2-Hexanone

Isopropylbenzene (Cumene)

p-Isopropyltoluene

Date: 11/11/2011 11:15 AM

Analytical Method: EPA 504.1 Preparation Method: EPA 504.1

ND ug/L

$0.029111 / 07 / 1100: 00 \quad 11 / 08 / 1101: 55 \quad 106-93-4$

Analytical Method: EPA 50308/8260

ND ug/L

ND ug/L

ND ug/L

ND ug/L

ND ug/L

ND ug/L

ND ug/L

ND ug/L

ND ug/L

ND $u g / L$

ND ug/L

ND ug/L

$3.4 \mathrm{ug} / \mathrm{L}$

ND ug/L

ND ug/L

ND ug/L

ND ug/L

ND ug/L

ND ug/L

ND ug/L

ND ug/L

ND ug/L

ND ug/L

ND ug/L

ND ug/L

ND ug/L

ND ug/L

ND ug/L

ND ug/L

ND ug/L

ND ug/L

ND ug/L

ND ug/L

ND ug/L

ND ug/L

ND ug/L

ND ug/L

ND ug/L

ND ug/L

ND ug/L

ND ug/L

ND ug/L

ND ug/L

ND ug/L

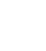

10.01

1.01

1.01

1.01

$1.0 \quad 1$

$1.0 \quad 1$

$1.0 \quad 1$

$10.0 \quad 1$

$1.0 \quad 1$

1.01

$1.0 \quad 1$

5.01

$1.0 \quad 1$

$1.0 \quad 1$

1.01

1.01

$1.0 \quad 1$

1.01

$1.0 \quad 1$

$2.5 \quad 1$

$1.0 \quad 1$

$1.0 \quad 1$

1.01

$1.0 \quad 1$

1.01

$1.0 \quad 1$

$1.0 \quad 1$

1.01

1.01

$1.0 \quad 1$

1.01

$1.0 \quad 1$

1.01

$1.0 \quad 1$

$1.0 \quad 1$

1.01

$1.0 \quad 1$

$1.0 \quad 1$

1.01

1.01

$1.0 \quad 1$

$10.0 \quad 1$

1.01

1.01

\begin{tabular}{|c|c|}
\hline $11 / 04 / 1121: 40$ & $67-64-1$ \\
\hline $11 / 04 / 1121: 40$ & $71-43-2$ \\
\hline $11 / 04 / 1121: 40$ & $108-86-1$ \\
\hline $11 / 04 / 1121: 40$ & $74-97-5$ \\
\hline $11 / 04 / 1121: 40$ & $75-27-4$ \\
\hline $11 / 04 / 1121: 40$ & $75-25-2$ \\
\hline $11 / 04 / 1121: 40$ & $74-83-9$ \\
\hline $11 / 04 / 1121: 40$ & $78-93-3$ \\
\hline $11 / 04 / 1121: 40$ & $104-51-8$ \\
\hline $11 / 04 / 1121: 40$ & $135-98-8$ \\
\hline $11 / 04 / 1121: 40$ & $98-06-6$ \\
\hline $11 / 04 / 1121: 40$ & $75-15-0$ \\
\hline $11 / 04 / 1121: 40$ & $56-23-5$ \\
\hline $11 / 04 / 1121: 40$ & $108-90-7$ \\
\hline $11 / 04 / 1121: 40$ & $75-00-3$ \\
\hline $11 / 04 / 1121: 40$ & $67-66-3$ \\
\hline $11 / 04 / 1121: 40$ & $74-87-3$ \\
\hline $11 / 04 / 1121: 40$ & $95-49-8$ \\
\hline $11 / 04 / 1121: 40$ & $106-43-4$ \\
\hline $11 / 04 / 1121: 40$ & $96-12-8$ \\
\hline $11 / 04 / 1121: 40$ & $124-48-1$ \\
\hline $11 / 04 / 1121: 40$ & $106-93-4$ \\
\hline $11 / 04 / 1121: 40$ & $74-95-3$ \\
\hline $11 / 04 / 1121: 40$ & $95-50-1$ \\
\hline $11 / 04 / 1121: 40$ & $541-73-1$ \\
\hline $11 / 04 / 1121: 40$ & $106-46-7$ \\
\hline $11 / 04 / 1121: 40$ & $75-71-8$ \\
\hline $11 / 04 / 1121: 40$ & $75-34-3$ \\
\hline $11 / 04 / 1121: 40$ & $107-06-2$ \\
\hline $11 / 04 / 1121: 40$ & $540-59-0$ \\
\hline $11 / 04 / 1121: 40$ & $75-35-4$ \\
\hline $11 / 04 / 1121: 40$ & $156-59-2$ \\
\hline $11 / 04 / 1121: 40$ & $156-60-5$ \\
\hline $11 / 04 / 1121: 40$ & $78-87-5$ \\
\hline $11 / 04 / 1121: 40$ & $142-28-9$ \\
\hline $11 / 04 / 1121: 40$ & $594-20-7$ \\
\hline $11 / 04 / 1121: 40$ & $563-58-6$ \\
\hline $11 / 04 / 1121: 40$ & $10061-01-5$ \\
\hline $11 / 04 / 1121: 40$ & $10061-02-6$ \\
\hline $11 / 04 / 1121: 40$ & $100-41-4$ \\
\hline $11 / 04 / 1121: 40$ & $87-68-3$ \\
\hline $11 / 04 / 1121: 40$ & $591-78-6$ \\
\hline $11 / 04 / 1121: 40$ & $98-82-8$ \\
\hline $11 / 04 / 1121: 40$ & $99-87-6$ \\
\hline
\end{tabular}

11/04/11 21:40 67-64-1

$11 / 04 / 1121: 40$ 108-86-1

11/04/11 21:40 74-97-5

$11 / 04 / 1121: 40 \quad 75-27-4$

$11 / 04 / 1121: 40 \quad 75-25-2$

11/04/1121:40 74-83-9

$11 / 04 / 1121: 40 \quad 104-51-8$

$11 / 04 / 1121: 40 \quad 135-98-8$

11/04/11 21:40 56-23-5

$11 / 04 / 1121: 40$ 108-90-7

$11 / 04 / 1121: 40$ 75-00-3

11/04/11 21:40 95-49-8

$11 / 04 / 1121: 40 \quad 106-43-4$

$11 / 04 / 1121: 40 \quad 96-12-8$

11/04/11 21:40 74-95-3

$11 / 04 / 1121: 40$ 95-50-1

$11 / 04 / 1121: 40$ 541-73-1

4/11 21:40 106-46-7

$11 / 04 / 1121: 40 \quad 75-34-3$

11/04/11 21:40 75-35-4

$11 / 04 / 1121: 40 \quad 156-59-2$

11/04/11 21:40 594-20-7

$11 / 04 / 1121: 40 \quad 563-58-6$

11/04/11 21:40 10061-01-5

$11 / 04 / 1121: 40 \quad 87-68-3$

$11 / 04 / 1121: 40 \quad 98-82-8$

This report shall not be reproduced, except in full,

without the written consent of Pace Analytical Services, Inc. 


\section{ANALYTICAL RESULTS}

Project: $\quad$ KS/MO Waste Water

Pace Project No.: 60109211

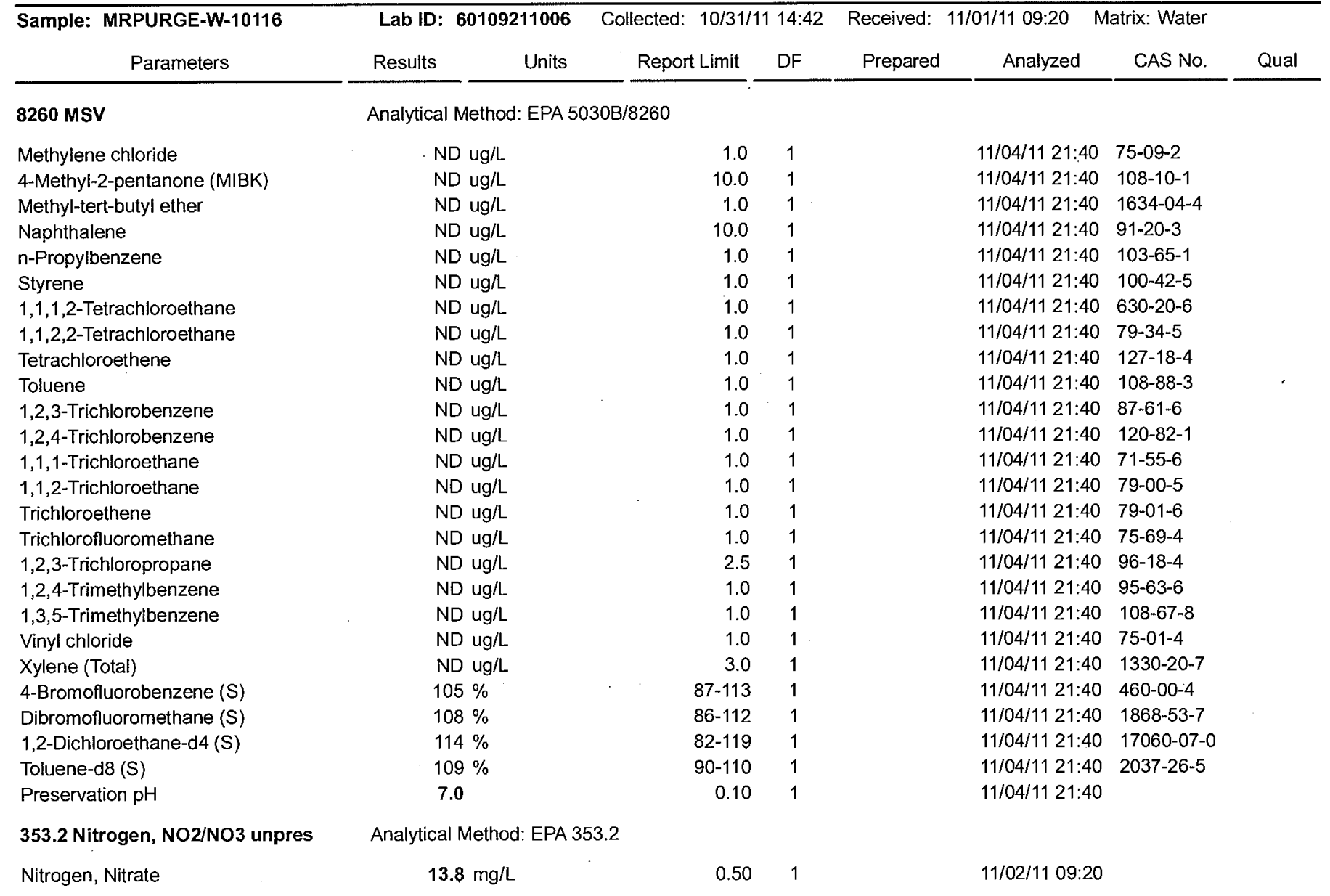




\section{ANALYTICAL RESULTS}

Project: $\quad$ KS/MO Waste Water

Pace Project No.: 60109211

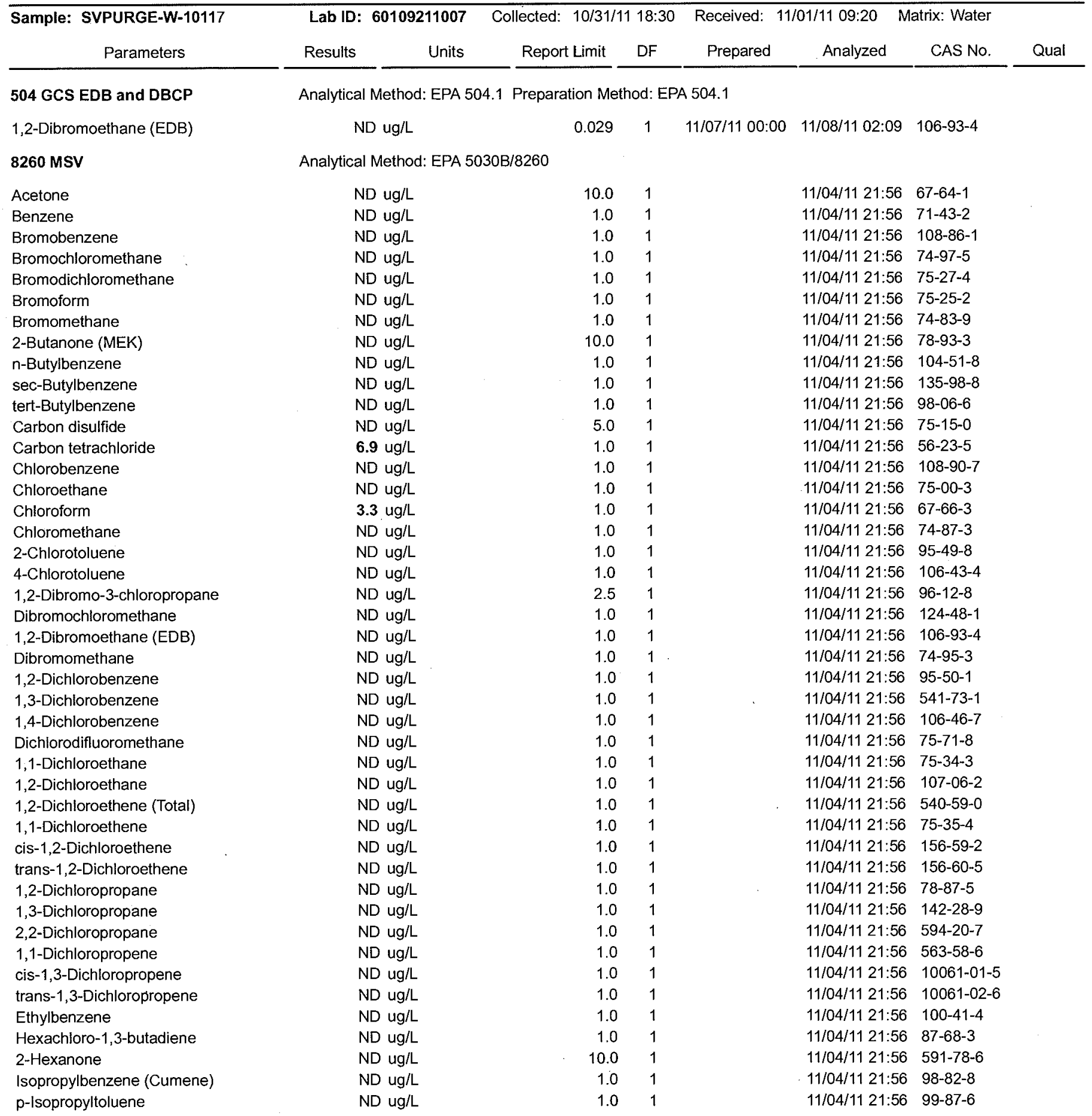

Date: 11/11/2011 11:15 AM 


\section{ANALYTICAL RESULTS}

Project: $\quad$ KS/MO Waste Water

Pace Project No.: 60109211

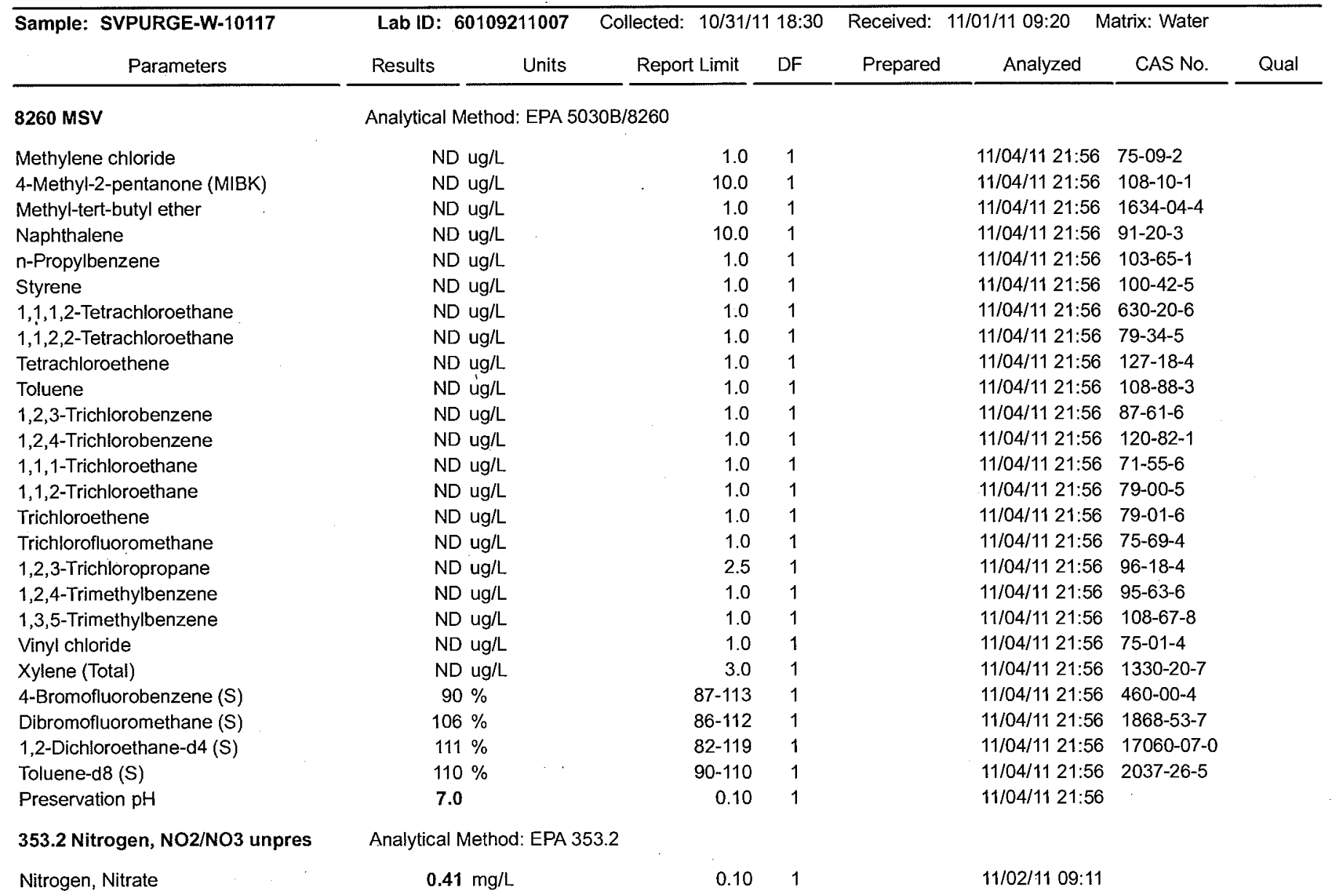




\section{QUALITY CONTROL DATA}

Project: KS/MO Waste Water

Pace Project No.: ' 60109211

\begin{tabular}{llll}
\hline QC Batch: & OEXT/31027 & Analysis Method: & EPA 504.1 \\
QC Batch Method: & EPA 504.1 & Analysis Description: & GCS 504 EDB DBCP
\end{tabular}

Associated Lab Samples: $\quad 60109211001,60109211002,60109211003,60109211004,60109211005,60109211006,60109211007$

METHOD BLANK: $906554 \quad$ Matrix: Water

Associated Lab Samples: $\quad 60109211001,60109211002,60109211003,60109211004,60109211005,60109211006,60109211007$

$\frac{\text { Parameter }}{1,2 \text {-Dibromoethane }(E D B)} \frac{\text { Units }}{u g / L} \frac{\text { Result }}{\text { ND }} \frac{\begin{array}{c}\text { Blank } \\ \text { Limit }\end{array}}{0.030} \frac{\text { Analyzed }}{11 / 07 / 1120: 35}-\frac{\text { Qualifiers }}{1}$

\begin{tabular}{|c|c|c|c|c|c|c|c|c|c|c|}
\hline \multirow[t]{2}{*}{ LABORATORY CONTROL SAMPLE \& LCSD: } & 906555 & \multicolumn{5}{|c|}{906556} & \multirow[b]{2}{*}{$\begin{array}{l}\% \operatorname{Rec} \\
\text { Limits }\end{array}$} & \multirow{2}{*}{\multicolumn{2}{|c|}{$\operatorname{Max}$}} & \multirow[b]{2}{*}{ Qualifiers } \\
\hline & Units & $\begin{array}{l}\text { Spike } \\
\text { Conc. }\end{array}$ & $\begin{array}{l}\text { LCS } \\
\text { Result }\end{array}$ & $\begin{array}{l}\text { LCSD } \\
\text { Result }\end{array}$ & $\begin{array}{l}\text { LCS } \\
\% \operatorname{Rec}\end{array}$ & $\begin{array}{l}\text { LCSD } \\
\% \operatorname{ReC}\end{array}$ & & & & \\
\hline
\end{tabular}




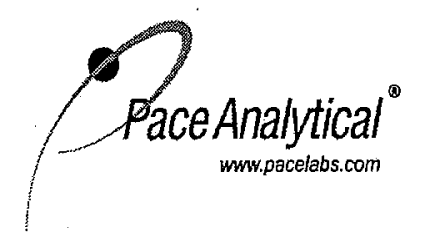

Pace Analytical Services, Inc.

9608 Loiret Blvd.

Lenexa, KS 66219

(913)599-5665

\section{QUALITY CONTROL DATA}

Project: KS/MO Waste Water

Pace Project No.: 60109211

\begin{tabular}{llll}
\hline QC Batch: & MSV $/ 41422$ & Analysis Method: & EPA 5030B/8260 \\
QC Batch Method: & EPA 5030B/8260 & Analysis Description: & 8260 MSV Water 7 day
\end{tabular}

Associated Lab Samples: $\quad 60109211001,60109211002,60109211003,60109211004,60109211005,60109211006,60109211007$

METHOD BLANK: $905182 \quad$ Matrix: Water

Associated Lab Samples: $\quad 60109211001,60109211002,60109211003,60109211004,60109211005,60109211006,60109211007$

\begin{tabular}{|c|c|c|c|c|c|}
\hline Parameter & Units & $\begin{array}{l}\text { Blank } \\
\text { Result }\end{array}$ & $\begin{array}{l}\text { Reporting } \\
\text { Limit }\end{array}$ & Analyzed & Qualifiers \\
\hline $1,1,1,2$-Tetrachloroethane & $\mathrm{ug} / \mathrm{L}$ & $\overline{N D}$ & 1.0 & $11 / 04 / 1117: 52$ & \\
\hline 1,1,1-Trichloroethane & $\mathrm{ug} / \mathrm{L}$ & ND & 1.0 & $11 / 04 / 1117: 52$ & \\
\hline 1,1,2,2-Tetrachloroethane & $\mathrm{ug} / \mathrm{L}$ & ND & 1.0 & $11 / 04 / 1117: 52$ & \\
\hline 1,1,2-Trichloroethane & $\mathrm{ug} / \mathrm{L}$ & ND & 1.0 & $11 / 04 / 1117: 52$ & \\
\hline 1,1-Dichloroethane & $\mathrm{ug} / \mathrm{L}$ & ND & $\cdot 1.0$ & $11 / 04 / 1117: 52$ & \\
\hline 1,1-Dichloroethene & $\mathrm{ug} / \mathrm{L}$ & ND & 1.0 & $11 / 04 / 1117: 52$ & \\
\hline 1,1-Dichloropropene & $\mathrm{ug} / \mathrm{L}$ & ND & 1.0 & $11 / 04 / 1117: 52$ & \\
\hline 1,2,3-Trichlorobenzene & $\mathrm{ug} / \mathrm{L}$ & ND & 1.0 & $11 / 04 / 1117: 52$ & \\
\hline 1,2,3-Trichloropropane & $\mathrm{ug} / \mathrm{L}$ & ND & 2.5 & $11 / 04 / 1117: 52$ & \\
\hline 1,2,4-Trichlorobenzene & $\mathrm{ug} / \mathrm{L}$ & ND & 1.0 & $11 / 04 / 1117: 52$ & \\
\hline 1,2,4-Trimethylbenzene & $\mathrm{ug} / \mathrm{L}$ & ND & 1.0 & $11 / 04 / 1117: 52$ & \\
\hline 1,2-Dibromo-3-chloropropane & $\mathrm{ug} / \mathrm{L}$ & ND & 2.5 & $11 / 04 / 1117: 52$ & \\
\hline 1,2-Dibromoethane (EDB) & $\mathrm{ug} / \mathrm{L}$ & ND & 1.0 & $11 / 04 / 1117: 52$ & \\
\hline 1,2-Dichlorobenzene & ug/L & ND & 1.0 & $11 / 04 / 1117: 52$ & \\
\hline 1,2-Dichloroethane & $\mathrm{ug} / \mathrm{L}$ & ND & 1.0 & $11 / 04 / 1117: 52$ & \\
\hline 1,2-Dichloroethene (Total) & $\mathrm{ug} / \mathrm{L}$ & ND & 1.0 & $11 / 04 / 1117: 52$ & \\
\hline 1,2-Dichloropropane & $\mathrm{ug} / \mathrm{L}$ & ND & 1.0 & $11 / 04 / 1117: 52$ & \\
\hline 1,3,5-Trimethylbenzene & $\mathrm{ug} / \mathrm{L}$ & ND & 1.0 & $11 / 04 / 1117: 52$ & \\
\hline 1,3-Dichlorobenzene & $\mathrm{ug} / \mathrm{L}$ & ND & 1.0 & $11 / 04 / 1117: 52$ & \\
\hline 1,3-Dichloropropane & $\mathrm{ug} / \mathrm{L}$ & ND & 1.0 & $11 / 04 / 1117: 52$ & \\
\hline 1,4-Dichlorobenzene & $\mathrm{ug} / \mathrm{L}$ & ND & 1.0 & $11 / 04 / 1117: 52$ & \\
\hline 2,2-Dichloropropane & $\mathrm{ug} / \mathrm{L}$ & $\mathrm{ND}$ & 1.0 & $11 / 04 / 1117: 52$ & \\
\hline 2-Butanone (MEK) & $\mathrm{ug} / \mathrm{L}$ & ND & 10.0 & $11 / 04 / 1117: 52$ & \\
\hline 2-Chlorotoluene & $\mathrm{ug} / \mathrm{L}$ & ND & 1.0 & $11 / 04 / 1117: 52$ & \\
\hline 2-Hexanone & $\mathrm{ug} / \mathrm{L}$ & ND & 10.0 & $11 / 04 / 1117: 52$ & \\
\hline 4-Chlorotoluene & $\mathrm{ug} / \mathrm{L}$ & ND & 1.0 & $11 / 04 / 1117: 52$ & \\
\hline 4-Methyl-2-pentanone (MIBK) & $\mathrm{ug} / \mathrm{L}$ & ND & 10.0 & $11 / 04 / 1117: 52$ & \\
\hline Acetone & $\mathrm{ug} / \mathrm{L}$ & ND & 10.0 & $11 / 04 / 1117: 52$ & \\
\hline Benzene & $\mathrm{ug} / \mathrm{L}$ & ND & 1.0 & $11 / 04 / 1117: 52$ & \\
\hline Bromobenzene & $\mathrm{ug} / \mathrm{L}$ & ND & 1.0 & $11 / 04 / 1117: 52$ & \\
\hline Bromochloromethane & $\mathrm{ug} / \mathrm{L}$ & ND & 1.0 & $11 / 04 / 1117: 52$ & \\
\hline Bromodichloromethane & $\mathrm{ug} / \mathrm{L}$ & ND & 1.0 & $11 / 04 / 1117: 52$ & \\
\hline Bromoform & $\mathrm{ug} / \mathrm{L}$ & ND & 1.0 & $11 / 04 / 1117: 52$ & \\
\hline Bromomethane & $\mathrm{ug} / \mathrm{L}$ & ND & 1.0 & $11 / 04 / 1117: 52$ & \\
\hline Carbon disulfide & $\mathrm{ug} / \mathrm{L}$ & ND & 5.0 & 11/04/11 17:52 & \\
\hline Carbon tetrachloride & $\mathrm{ug} / \mathrm{L}$ & ND & 1.0 & $11 / 04 / 1117: 52$ & \\
\hline Chlorobenzene & $\mathrm{ug} / \mathrm{L}$ & ND & 1.0 & $11 / 04 / 1117: 52$ & \\
\hline Chloroethane & $\mathrm{ug} / \mathrm{L}$ & ND & 1.0 & $11 / 04 / 1117: 52$ & \\
\hline Chloroform & ug/L & ND & 1.0 & $11 / 04 / 1117: 52$ & \\
\hline Chloromethane & ug/L & ND & 1.0 & $11 / 04 / 1117: 52$ & \\
\hline cis-1,2-Dichloroethene & ug/L & ND & 1.0 & $11 / 04 / 1117: 52$ & \\
\hline cis-1,3-Dichloropropene & ug/L & ND & 1.0 & $11 / 04 / 1117: 52$ & \\
\hline Dibromochloromethane & $\mathrm{ug} / \mathrm{L}$ & ND & 1.0 & $11 / 04 / 1117: 52$ & \\
\hline
\end{tabular}

Date: 11/11/2011 11:15 AM 


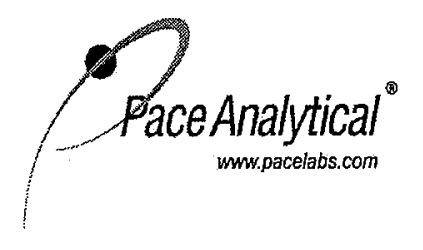

Pace Analytical Services, Inc.

9608 Loiret Blvod.

Lenexa, KS 66219

(913)599-5665

\section{QUALITY CONTROL DATA}

Project: KS/MO Waste Water

Pace Project No.: 60109211

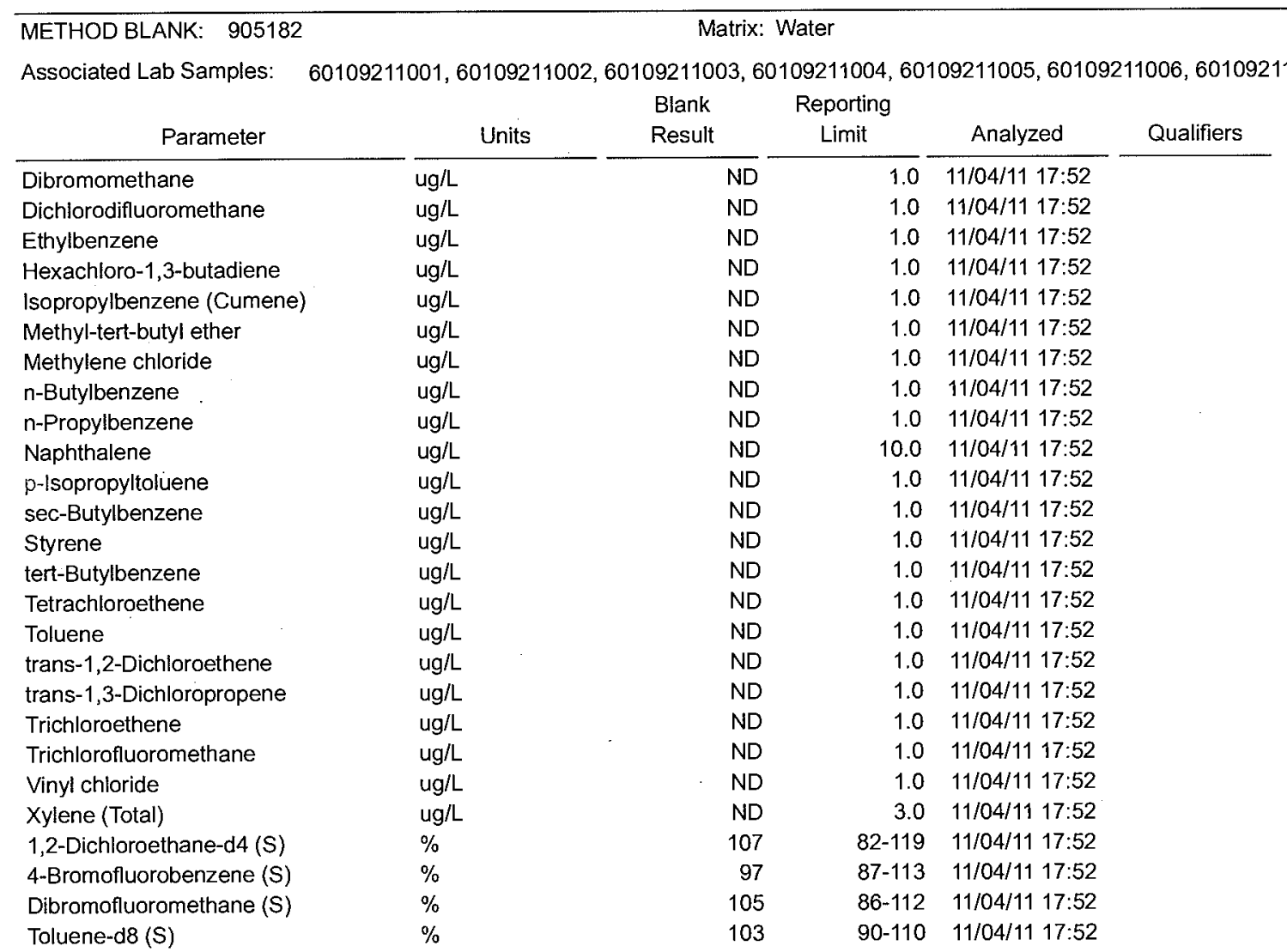

\begin{tabular}{|c|c|c|c|c|c|c|}
\hline \multirow{2}{*}{$\begin{array}{c}\text { LABORATORY CONTROL SAMPLE: } \\
\text { Parameter }\end{array}$} & \multicolumn{6}{|l|}{905183} \\
\hline & Units & $\begin{array}{l}\text { Spike } \\
\text { Conc. }\end{array}$ & $\begin{array}{l}\text { LCS } \\
\text { Result }\end{array}$ & $\begin{array}{l}\text { LCS } \\
\% \operatorname{Rec}\end{array}$ & $\begin{array}{l}\% \text { Rec } \\
\text { Limits }\end{array}$ & Qualifiers \\
\hline 1,1,1,2-Tetrachloroethane & $\mathrm{ug} / \mathrm{L}$ & 20 & 17.3 & 86 & $81-121$ & \\
\hline 1,1,1-Trichloroethane & $\mathrm{ug} / \mathrm{L}$ & 20 & 17.6 & 88 & $82-119$ & \\
\hline 1,1,2,2-Tetrachloroethane & $u g / L$ & 20 & 18.1 & 91 & $78-124$ & \\
\hline 1,1,2-Trichloroethane & $\mathrm{ug} / \mathrm{L}$ & 20 & 20.3 & 102 & $79-121$ & \\
\hline 1,1-Dichloroethane & $\mathrm{ug} / \mathrm{L}$ & 20 & 18.3 & 91 & $73-119$ & \\
\hline 1,1-Dichloroethene & $\mathrm{ug} / \mathrm{L}$ & 20 & 17.7 & 89 & $75-120$ & \\
\hline 1,1-Dichloropropene & $u g / L$ & 20 & 18.5 & 93 & $79-123$ & \\
\hline 1,2,3-Trichlorobenzene & $\mathrm{ug} / \mathrm{L}$ & 20 & 17.8 & 89 & $73-122$ & \\
\hline 1,2,3-Trichloropropane & $\mathrm{ug} / \mathrm{L}$ & 20 & 18.1 & 91 & $77-124$ & . \\
\hline 1,2,4-Trichlorobenzene & $\mathrm{ug} / \mathrm{L}$ & 20 & 17.4 & 87 & $75-120$ & \\
\hline $1,2,4$-Trimethylbenzene & $\mathrm{ug} / \mathrm{L}$ & 20 & 18.7 & 94 & $77-120$ & \\
\hline 1,2-Dibromo-3-chloropropane & $u g / L$ & 20 & 16.7 & 84 & $69-125$ & \\
\hline 1,2-Dibromoethane (EDB) & $\mathrm{ug} / \mathrm{L}$ & 20 & 18.8 & 94 & $85-121$ & \\
\hline 1,2-Dichlorobenzene & $\mathrm{ug} / \mathrm{L}$ & 20 & 19.2 & 96 & $82-115$ & \\
\hline 1,2-Dichloroethane & $u g / L$ & 20 & 19.3 & 96 & $77-125$ & \\
\hline
\end{tabular}

Date: 11/11/2011 11:15 AM 


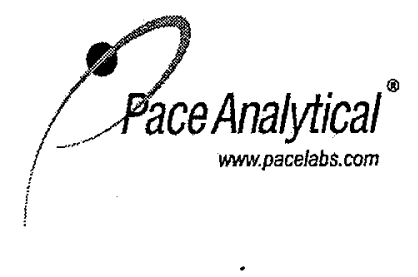

Pace Analytical Services, inc. 9608 Loiret Blvd. Lenexa, KS 66219

(913)599-5665

\section{QUALITY CONTROL DATA}

Project: KS/MO Waste Water

Pace Project No.: 60109211

\begin{tabular}{|c|c|c|c|c|c|c|}
\hline \multirow{2}{*}{$\begin{array}{c}\text { LABORATORY CONTROL SAMPLE: } \\
\text { Parameter }\end{array}$} & \multicolumn{6}{|l|}{905183} \\
\hline & Units & $\begin{array}{l}\text { Spike } \\
\text { Conc. }\end{array}$ & $\begin{array}{c}\text { LCS } \\
\text { Result }\end{array}$ & $\begin{array}{c}\text { LCS } \\
\% \operatorname{Rec}\end{array}$ & $\begin{array}{l}\% \operatorname{Rec} \\
\text { Limits }\end{array}$ & Qualifiers \\
\hline 1,2-Dichloroethene (Total) & $\mathrm{ug} / \mathrm{L}$ & 40 & 40.0 & 100 & $79-120$ & \\
\hline 1,2-Dichloropropane & $\mathrm{ug} / \mathrm{L}$ & 20 & 18.8 & 94 & $83-119$ & \\
\hline 1,3,5-Trimethylbenzene & $\mathrm{ug} / \mathrm{L}$ & 20 & 18.1 & 91 & $79-121$ & \\
\hline 1,3-Dichlorobenzene & $\mathrm{ug} / \mathrm{L}$ & 20 & 17.7 & 88 & $79-117$ & \\
\hline 1,3-Dichloropropane & $u g / L$ & 20 & 19.2 & 96 & $78-116$ & \\
\hline 1,4-Dichlorobenzene & ug/L & 20 & 18.7 & 94 & $83-115$ & \\
\hline 2,2-Dichloropropane & $\mathrm{ug} / \mathrm{L}$ & 20 & 16.3 & 82 & $66-123$ & \\
\hline 2-Butanone (MEK) & $u g / L$ & 100 & 101 & 101 & $43-165$ & \\
\hline 2-Chlorotoluene & $u g / L$ & 20 & 18.6 & 93 & $81-117$ & \\
\hline 2-Hexanone & $\mathrm{ug} / \mathrm{L}$ & 100 & 98.3 & 98 & $47-159$ & \\
\hline 4-Chlorotoluene & $u g / L$ & 20 & 18.8 & 94 & $84-116$ & \\
\hline 4-Methyl-2-pentanone (MIBK) & $\mathrm{ug} / \mathrm{L}$ & 100 & 90.7 & 91 & $71-129$ & \\
\hline Acetone & ug/L & 100 & 111 & 111 & $18-192$ & \\
\hline Benzene & $\mathrm{ug} / \mathrm{L}$ & 20 & 19.5 & 97 & $82-117$ & \\
\hline Bromobenzene & ug/L & 20 & 18.5 & 92 & $83-116$ & \\
\hline Bromochloromethane & $\mathrm{ug} / \mathrm{L}$ & 20 & 18.9 & 94 & $79-121$ & . \\
\hline Bromodichloromethane & $u g / L$ & 20 & 18.5 & 92 & $79-114$ & \\
\hline Bromoform & $u g / L$ & 20 & 17.8 & 89 & $78-121$ & \\
\hline Bromomethane & $\mathrm{ug} / \mathrm{L}$ & 20 & 20.6 & 103 & $36-146$ & \\
\hline Carbon disulfide & $\mathrm{ug} / \mathrm{L}$ & 20 & 20.6 & 103 & $75-138$ & \\
\hline Carbon tetrachloride & $u g / L$ & 20 & 19.3 & 96 & $80-123$ & \\
\hline Chlorobenzene & $u g / L$ & 20 & 18.2 & 91 & $83-121$ & \\
\hline Chloroethane & $\mathrm{ug} / \mathrm{L}$ & 20 & 19.6 & 98 & $42-166$ & \\
\hline Chloroform & ug/L & 20 & 20.1 & 100 & $82-116$ & \\
\hline Chloromethane & $\mathrm{ug} / \mathrm{L}$ & 20 & 17.7 & 88 & $32-127$ & \\
\hline cis-1,2-Dichloroethene & $u g / L$ & 20 & 18.2 & 91 & $80-119$ & \\
\hline cis-1,3-Dichloropropene & $\mathrm{ug} / \mathrm{L}$ & 20 & 17.0 & 85 & $76-119$ & \\
\hline Dibromochloromethane & $u g / L$ & 20 & 17.9 & 89 & $81-123$ & \\
\hline Dibromomethane & $\mathrm{ug} / \mathrm{L}$ & 20 & 19.2 & 96 & $79-123$ & \\
\hline Dichlorodifluoromethane & $\mathrm{ug} / \mathrm{L}$ & 20 & 15.2 & 76 & $10-163$ & \\
\hline Ethylbenzene & $\mathrm{ug} / \mathrm{L}$ & 20 & 17.7 & 88 & $79-121$ & \\
\hline Hexachloro-1,3-butadiene & ug/L & 20 & 18.5 & 92 & $78-125$ & \\
\hline Isopropylbenzene (Cumene) & $\mathrm{ug} / \mathrm{L}$ & 20 & 18.7 & 93 & $80-120$ & \\
\hline Methyl-tert-butyl ether & $\mathrm{ug} / \mathrm{L}$ & 20 & 18.1 & 91 & $78-119$ & \\
\hline Methylene chloride & $\mathrm{ug} / \mathrm{L}$ & 20 & 19.6 & 98 & $75-118$ & \\
\hline n-Butylbenzene & $\mathrm{ug} / \mathrm{L}$ & 20 & 18.2 & 91 & $80-126$ & \\
\hline n-Propylbenzene & $\mathrm{ug} / \mathrm{L}$ & 20 & 18.3 & 91 & $83-116$ & \\
\hline Naphthalene & $\mathrm{ug} / \mathrm{L}$ & 20 & 16.8 & 84 & $66-133$ & \\
\hline p-lsopropyltoluene & $\mathrm{ug} / \mathrm{L}$ & 20 & 17.9 & 89 & $77-120$ & \\
\hline sec-Butylbenzene & $\mathrm{ug} / \mathrm{L}$ & 20 & 17.8 & 89 & $81-120$ & \\
\hline Styrene & $\mathrm{ug} / \mathrm{L}$ & 20 & 18.8 & 94 & $84-115$ & \\
\hline tert-Butylbenzene & ug/L & 20 & 18.0 & 90 & $80-117$ & \\
\hline Tetrachloroethene & ug/L & 20 & 19.8 & 99 & $80-124$ & \\
\hline Toluene & $\mathrm{ug} / \mathrm{L}$ & 20 & 19.1 & 95 & $80-120$ & \\
\hline trans-1,2-Dichloroethene & $\mathrm{ug} / \mathrm{L}$ & 20 & 21.8 & 109 & $79-120$ & \\
\hline trans-1,3-Dichloropropene & $\mathrm{ug} / \mathrm{L}$ & 20 & 18.5 & 92 & $76-118$ & \\
\hline Trichloroethene & $u g / L$ & 20 & 17.5 & 88 & $76-122$ & \\
\hline Trichlorofluoromethane & $\mathrm{ug} / \mathrm{L}$ & 20 & 19.0 & 95 & $72-120$ & \\
\hline
\end{tabular}

Date: $11 / 11 / 201111: 15$ AM 


\section{QUALITY CONTROL DATA}

Project: $\quad$ KS/MO Waste Water

Pace Project No.: $\quad 60109211$

\begin{tabular}{|c|c|c|c|c|c|c|}
\hline LABORATORY CONTROL & 905183 & & & & & \\
\hline Parameter & Units & $\begin{array}{l}\text { Spike } \\
\text { Conc. }\end{array}$ & $\begin{array}{l}\text { LCS } \\
\text { Result }\end{array}$ & $\begin{array}{c}\text { LCS } \\
\% \operatorname{ReC}\end{array}$ & $\begin{array}{l}\% \operatorname{Rec} \\
\text { Limits }\end{array}$ & Qualifiers \\
\hline Vinyl chloride & $\mathrm{ug} / \mathrm{L}$ & 20 & 17.5 & 88 & $57-163$ & \\
\hline Xylene (Total) & ug/L & 60 & 53.9 & 90 & $75-120$ & \\
\hline 1,2-Dichloroethane-d4 (S) & $\%$ & & & 103 & $82-119$ & \\
\hline 4-Bromofluorobenzene (S) & $\%$ & & & 104 & $87-113$ & \\
\hline Dibromofluoromethane (S) & $\%$ & & & 101 & $86-112$ & \\
\hline Toluene-d8 (S) & $\%$ & & & 99 & $90-110$ & \\
\hline
\end{tabular}




\section{QUALITY CONTROL DATA}

Project: $\quad$ KS/MO Waste Water

Pace Project No.: 60109211

\begin{tabular}{llll}
\hline QC Batch: & WETA $/ 18128$ & Analysis Method: & EPA 353.2 \\
QC Batch Method: & EPA 353.2 & Analysis Description: & 353.2 Nitrate + Nitrite, Unpres.
\end{tabular}

Associated Lab Samples: 60109211001

METHOD BLANK: 903260

Associated Lab Samples: $\quad 60109211001$

$\frac{\text { Parameter }}{\text { Nitrogen, Nitrate }} \frac{\text { Units }}{\mathrm{mg} / \mathrm{L}} \frac{\begin{array}{c}\text { Blank } \\ \text { Result }\end{array}}{\mathrm{ND}} \frac{\begin{array}{c}\text { Reporting } \\ \text { Limit }\end{array}}{0.10} \frac{\text { Analyzed }}{11 / 02 / 1108: 39} \frac{\text { Qualifiers }}{1}$

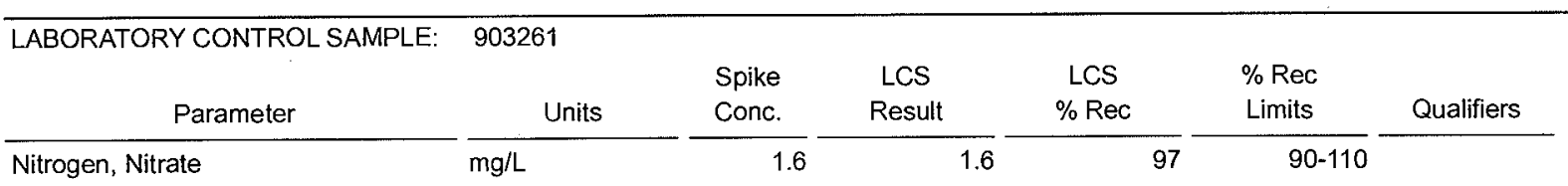

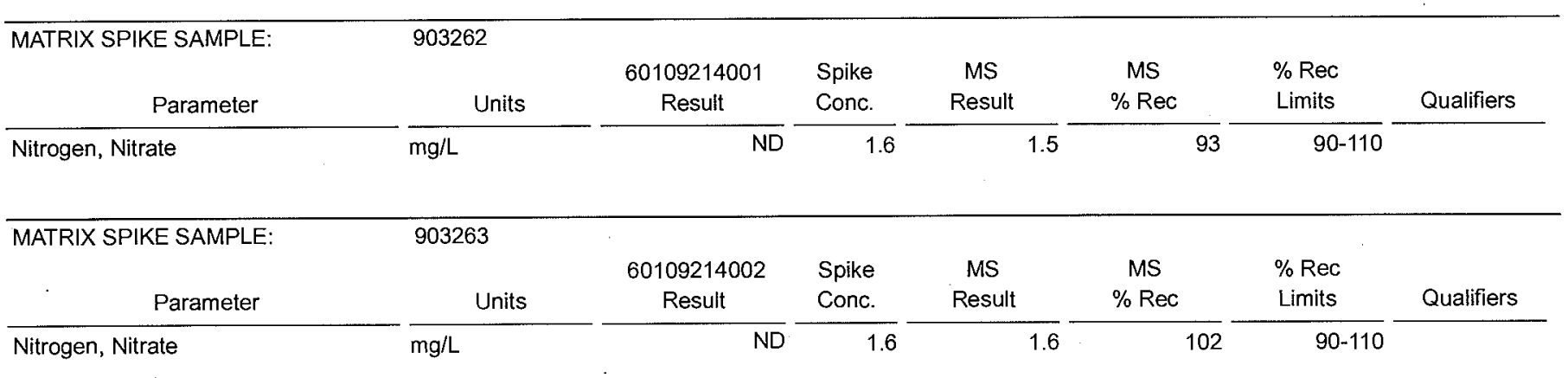

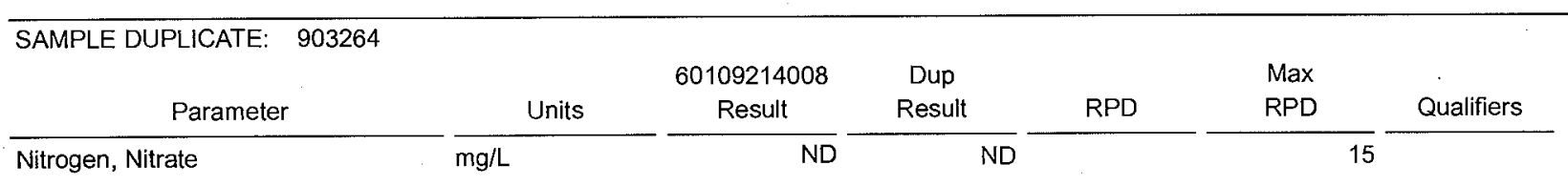




\section{QUALITY CONTROL DATA}

Project: $\quad$ KS/MO Waste Water

Pace Project No.: 60109211

\begin{tabular}{|c|c|c|c|}
\hline QC Batch: & WETA 18129 & Analysis Method: & EPA 353.2 \\
\hline QC Batch Method: & EPA 353.2 & Analysis Description: & 353.2 Nitrate + Nitrite, Unpres. \\
\hline
\end{tabular}

Associated Lab Samples: $\quad 60109211002,60109211003,60109211004,60109211005,60109211006,60109211007$

METHOD BLANK: $903266 \quad$ Matrix: Water

Associated Lab Samples: $\quad 60109211002,60109211003,60109211004,60109211005,60109211006,60109211007$

\begin{tabular}{|c|c|c|c|c|c|}
\hline Parameter & Units & $\begin{array}{l}\text { Blank } \\
\text { Result }\end{array}$ & $\begin{array}{c}\text { Reporting } \\
\text { Limit }\end{array}$ & Analyzed & Qualifiers \\
\hline Nitrogen, Nitrate & $\overline{\mathrm{mg} / \mathrm{L}}$ & $\overline{N D}$ & 0.10 & $11 / 02 / 1109: 03$ & \\
\hline
\end{tabular}

\begin{tabular}{|c|c|c|c|c|c|c|}
\hline LABORATORY CONTROL SAMPLE: & 903267 & & & & & \\
\hline Parameter & Units & $\begin{array}{l}\text { Spike } \\
\text { Conc. }\end{array}$ & $\begin{array}{l}\text { LCS } \\
\text { Result }\end{array}$ & $\begin{array}{l}\text { LCS } \\
\% \operatorname{Rec}\end{array}$ & $\begin{array}{l}\% \text { Rec } \\
\text { Limits }\end{array}$ & Qualifiers \\
\hline Nitrogen, Nitrate & $\mathrm{mg} / \mathrm{L}$ & $\overline{1.6}$ & 1.6 & 98 & $90-110$ & \\
\hline
\end{tabular}

\begin{tabular}{|c|c|c|c|c|c|c|c|}
\hline MATRIX SPIKE SAMPLE: & 903268 & & & & & & \\
\hline Parameter & Uhits & $\begin{array}{c}60109211007 \\
\text { Result }\end{array}$ & $\begin{array}{l}\text { Spike } \\
\text { Conc. }\end{array}$ & $\begin{array}{c}\text { MS } \\
\text { Result }\end{array}$ & $\begin{array}{c}\text { MS } \\
\% \operatorname{Rec}\end{array}$ & $\begin{array}{l}\% \text { Rec } \\
\text { Limits }\end{array}$ & Qualifiers \\
\hline Nitrogen, Nitrate & $\overline{\mathrm{mg} / \mathrm{L}}$ & 0.4 & 1.6 & 1.9 & 95 & $90-110$ & \\
\hline
\end{tabular}

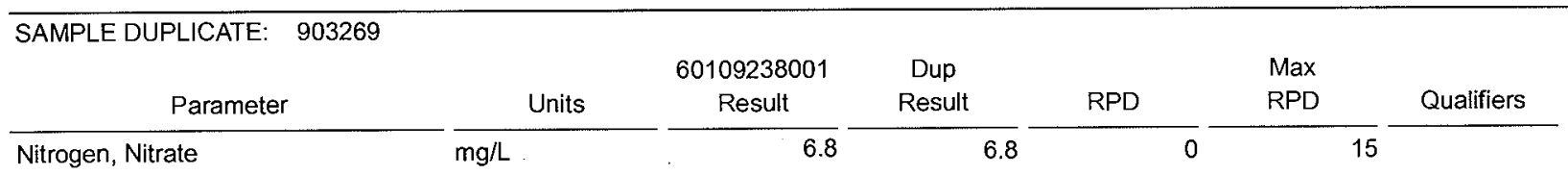




\section{QUALIFIERS}

Project: KS/MO Waste Water

Pace Project No.: 60109211

\section{DEFINITIONS}

DF - Dilution Factor, if reported, represents the factor applied to the reported data due to changes in sample preparation, dilution of the sample aliquot, or moisture content.

ND - Not Detected at or above adjusted reporting limit.

$\mathrm{J}$ - Estimated concentration above the adjusted method detection limit and below the adjusted reporting limit.

MDL - Adjusted Method Detection Limit.

$S$ - Surrogate

1,2-Diphenyihydrazine (8270 listed analyte) decomposes to Azobenzene.

Consistent with EPA guidelines, unrounded data are displayed and have been used to calculate \% recovery and RPD values.

LCS(D) - Laboratory Control Sample (Duplicate)

MS(D) - Matrix Spike (Duplicate)

DUP - Sample Duplicate

RPD - Rejative Percent Difference

NC - Not Calculable.

SG - Silica Gel - Clean-Up

$\mathrm{U}$ - Indicates the compound was analyzed for, but not detected.

$\mathrm{N}$-Nitrosodiphenylamine decomposes and cannot be separated from Diphenylamine using Method 8270 . The result reported for each analyte is a combined concentration.

Pace Analytical is TNI accredited. Contact your Pace PM for the current list of accredited analytes.

\section{BATCH QUALIFIERS}

Batch: MSV/41422

[M5] A matrix spike/matrix spike duplicate was not performed for this batch due to insufficient sample volume. 


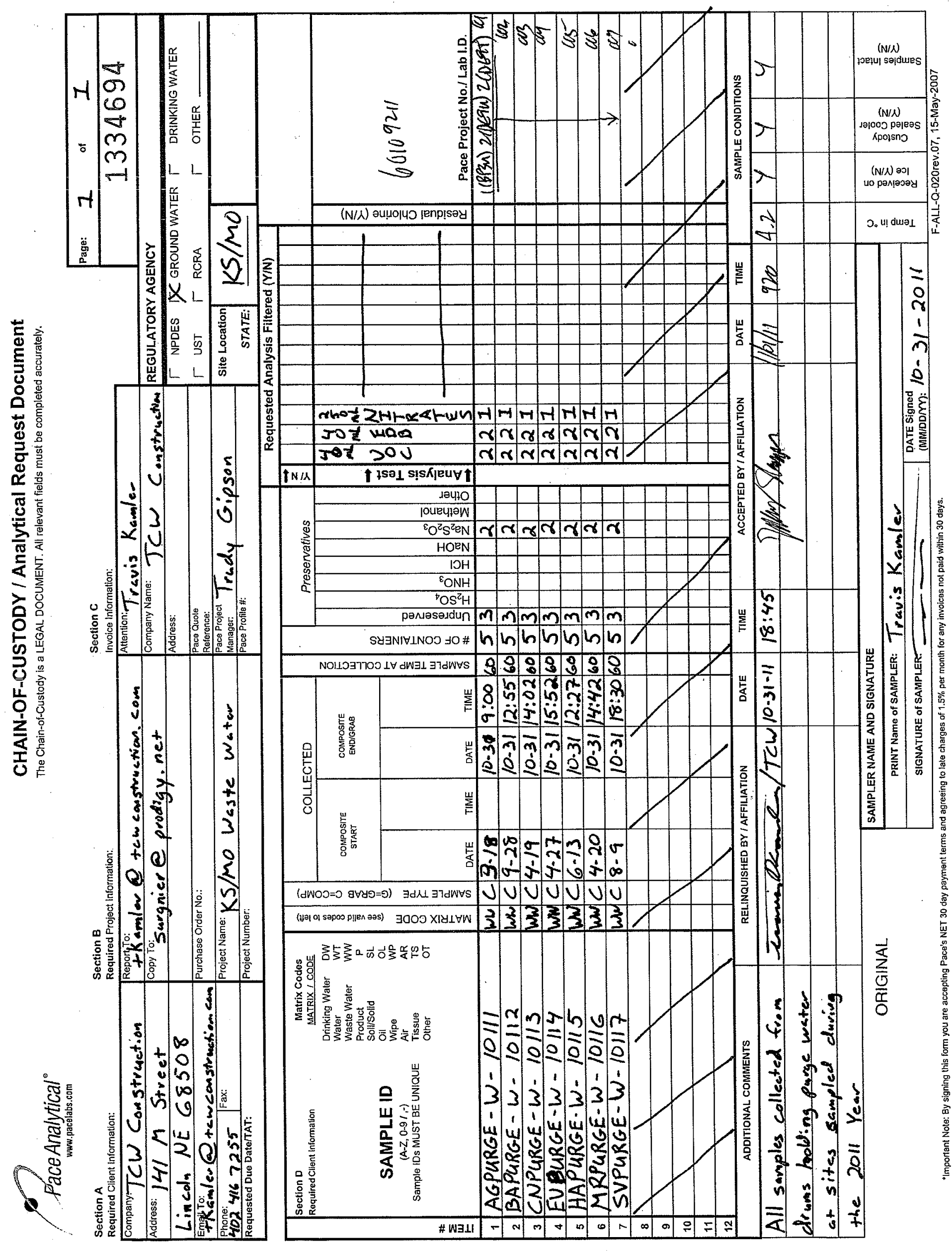


Client Name: TCW Const.

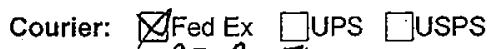

Tracking \#: 875827463563

Custody Seal on Cooler/Box Present

$\square$ client $\square$ commercial $\square$ Pace $\square$ other
Pace Shipping Label Used? $\square$ Yes $\square$ Noo
$\bigotimes$ Yes $\square$ No Seals intact: $\square$ Yes $\square$ No

Project \# 60109211

Packing Material: \Bubble Wrap $\square$ Bubble Bags $\square$ Foam . $\square$ None $\square$ Dther Thermometer Used: T-199 / T-194 Type of lce: Whe None $\square$ Samples on ice, cooling process has begun

Cooler Temperature: $\quad 4,2$ Temperature should be above freezing to $6^{\circ} \mathrm{C}$ Comments:

\begin{tabular}{l}
\hline Optional \\
\hline $\begin{array}{l}\text { Proj. Due Date: } \\
\text { Proj. Name: }\end{array}$ \\
\hline
\end{tabular}

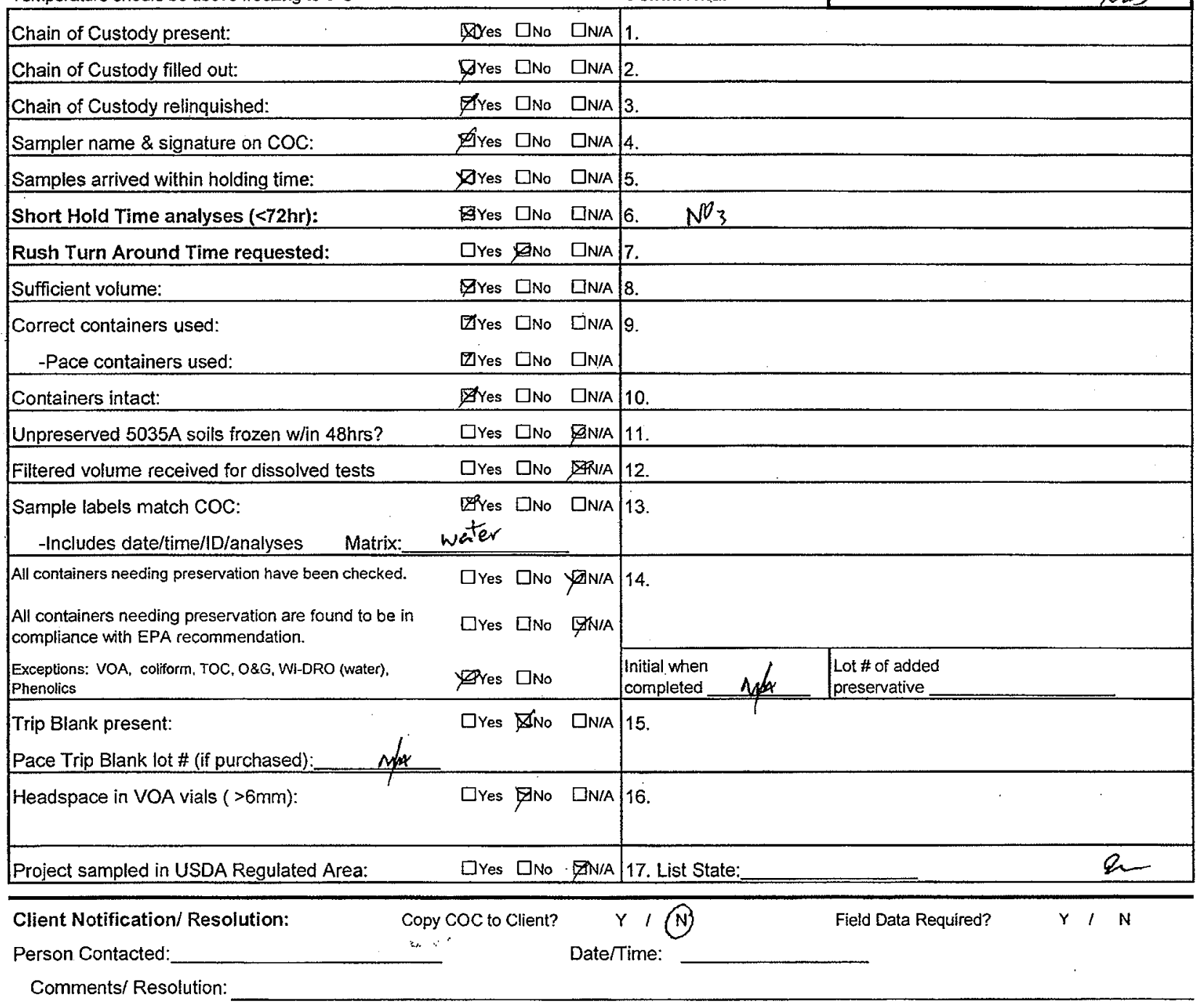
Date and initials of person examining contents: $1 \mathrm{ND} 1 / 1 / \mathrm{h} / 025$

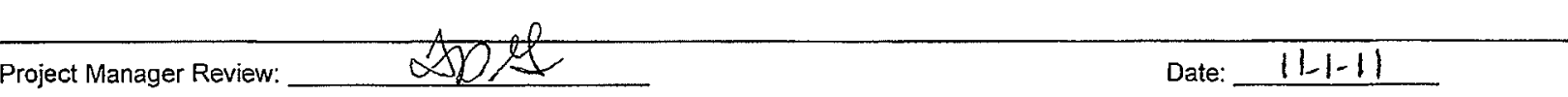

Note: Whenever there is a discrepancy affecting North Carolina compliance samples, a copy of this form will be sent to the North Carolina DEHNR Certification Office (i.e out of hold, incorrect preservative, out of temp, incorrect containers) 


\section{Supplement 2:}

Sample Documentation from TestAmerica Laboratories, Inc. 


\section{TestAmerica}

THE LEADER IN ENVIRONMENTAL TESTING

\section{ANALYTICAL REPORT}

Job Number: 200-7268-1

SDG Number: 200-7268

Job Description: Barnes (200-7268)

Contract Number: 1E-30401

For:

Argonne National Laboratory

9700 South Cass Avenue

Building 203

Office B-149

Argonne, IL 60439

Attention: Mr. Clyde Dennis

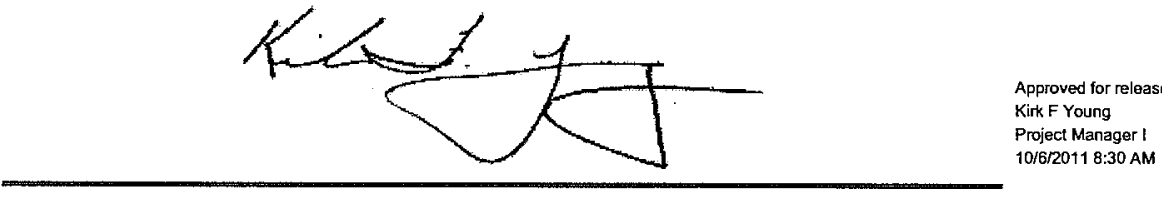

Kirk F Young

Project Manager I

kirk.young@testamericainc.com

$10 / 06 / 2011$

The test results in this report relate only to sample(s) as received by the laboratory. These test results were derived under a quality system that adheres to the requirements of NELAC. Pursuant to NELAC, this report may not be produced in full without written approval from the laboratory 


\section{Table of Contents}

Cover Title Page $\ldots \ldots \ldots \ldots \ldots \ldots \ldots \ldots \ldots \ldots \ldots \ldots$

Report Narrative . . . . . . . . . . . . . . . . . . . 4

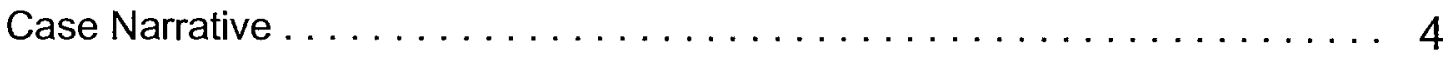

Qualifier Definition ............................. 6

External Chain of Custody . . . . . . . . . . . . . . 7

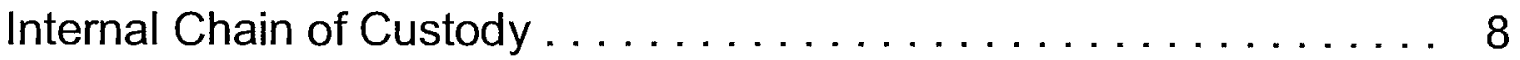

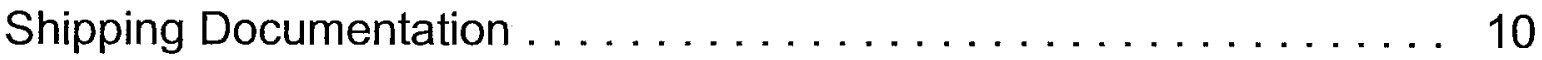

Airbills (if Applicable) . . . . . . . . . . . . . . . . . . . 11

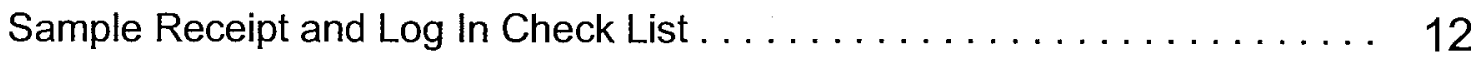

Methodology Review . . . . . . . . . . . . . . . . . . . . . 14

QC Summary - SOM01.2 Volatiles-Trace . . . . . . . . . . . . . 15

QC Summary - SOM01.2 Volatiles-Trace . . . . . . . . . . . . . 15

Deuterated Monitoring Compound Summary $\ldots \ldots \ldots \ldots \ldots \ldots \ldots \ldots \ldots \ldots \ldots \ldots$

Method Blank $\ldots \ldots \ldots \ldots \ldots \ldots \ldots \ldots \ldots \ldots \ldots \ldots \ldots \ldots, 17$

GC/MS Instrument Performance Check $\ldots \ldots \ldots \ldots \ldots \ldots \ldots \ldots \ldots \ldots \ldots \ldots \ldots \ldots$

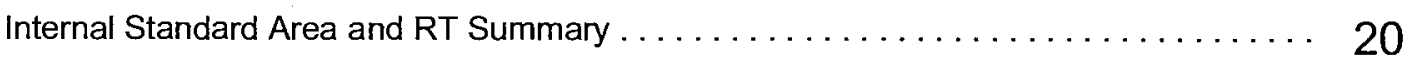

Sample Data - SOM01.2 Volatiles-Trace . . . . . . . . . . . . . . 21

Sample Data - SOM01.2 Volatiles-Trace . . . . . . . . . . . . . 21

BAMW13S-W-28814 ........................................ 21

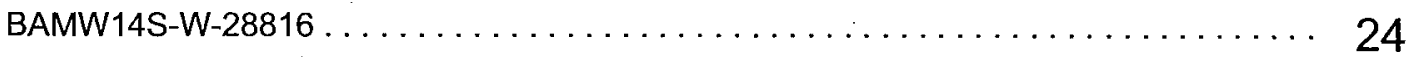

BAMW17-W-28817 $\ldots \ldots \ldots \ldots \ldots \ldots \ldots \ldots \ldots \ldots \ldots \ldots \ldots \ldots \ldots \ldots \ldots \ldots \ldots \ldots \ldots, 27$

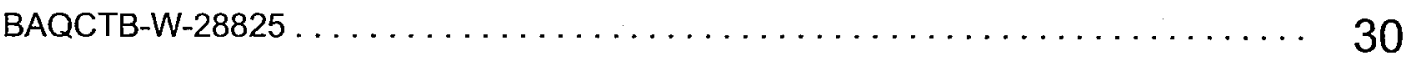

Standards - SOM01.2 Volatiles-Trace . . . . . . . . . . . . . . 33

Standards - SOM01.2 Volatiles-Trace . . . . . . . . . . . . . . 33

Initial Calibration Data $\ldots \ldots \ldots \ldots \ldots \ldots \ldots \ldots \ldots \ldots \ldots \ldots \ldots \ldots \ldots \ldots, 33$

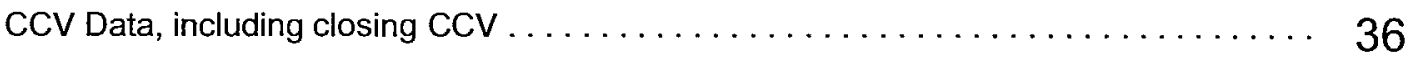




\section{Table of Contents}

Raw Qc Data - SOM01.2 Volatiles-Trace . . . . . . . . . . . . . . . 42

Raw Qc Data - SOM01.2 Volatiles-Trace . . . . . . . . . . . . . . 42

Raw Qc Data - SOM01.2 Volatiles-Trace . . . . . . . . . . . . . . . 42

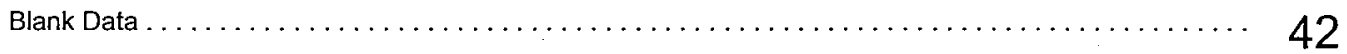




\title{
CASE NARRATIVE
}

\section{Client: Argonne National Laboratory}

\section{Project: Barnes (200-7268)}

\author{
Report Number: 200-7268-1
}

Enclosed is the data set for the referenced project work. With the exceptions noted as flags or footnotes, standard analytical protocols were followed in performing the analytical work and the applied control limits were met.

Calculations were performed before rounding to avoid round-off errors in calculated results.

All holding times were met and proper preservation noted for the methods performed on these samples, unless otherwise detailed in the individual sections below.

\section{Receipt}

The samples were received on 09/30/2011. Documentation of the condition of the samples at the time of their receipt and any exception to the laboratory's Sample Acceptance Policy is documented in the Shipping and Receiving section of this submittal. The samples, as received, were not acid preserved. On that basis, the laboratory did provide for the analytical work to be performed within seven days of sample collection.

\section{SOM01.2 Volatile Organics (Trace Level Water)}

A storage blank was prepared for volatile organics analysis, and stored in association with the storage of the samples. That storage blank, identified as VHBLK01, was carried through the holding period with the samples, and analyzed.

Each sample in the sample set was analyzed without a dilution. Each of the analyses associated with the sample set exhibited an acceptable internal standard performance. There was an acceptable recovery of each deuterated monitoring compound (DMC) in the analysis of the method blank associated with the analytical work, and in the analysis of the storage blank associated with the sample set. The analysis of the samples in this sample set did meet the technical acceptance criteria specific to DMC recoveries, although not all DMC recoveries were within the control range in each analysis. The technical acceptance criteria does provide for the recovery of up to three DMCs to fall outside of the control range in the analysis of field samples. Matrix spike and matrix spike duplicate analyses were not performed on samples in this sample set. Trace concentrations of acetone, carbon disulfide, toluene, 1,3-dichlorobenzene, and 1,2,4-trichlorobenzene were identified in the analysis of the method blank associated with the analytical work. The concentration of each analyte in that analysis was below the established reporting limit, and the analysis did meet the technical acceptance criteria for a compliant method blank analysis. A trace concentration of acetone was identified in the analysis of the storage blank associated with the sample set. The concentration of acetone in that analysis was below the established reporting limit, and the analysis did meet the technical acceptance criteria for a compliant storage blank analysis. Present in the method blank and storage blank analyses was a non-target constituent that represents a compound that is related to the DMC formulation. The fact that the presence of this compound is not within the laboratory's control is at issue. The derived results for that compound have been qualified with an " $X$ " qualifier to reflect the source of the contamination. 
The responses for each of the target analytes met the relative standard deviation criterion in the initial calibration. The response for each target analyte met the percent difference criterion in the opening/continuing calibration check acquisition. The response for each target analyte met the 50.0 percent difference criterion in the closing calibration check acquisition.

The primary quantitation mass for methylcyclohexane that is specified in the Statement of Work is mass 83 . The laboratory did identify a contribution to mass 83 from 1,2-dichloropropane- $\mathrm{d}_{6}$, one of the deuterated monitoring compounds (DMCs). The laboratory did change the primary quantitation mass assignment to mass 55 for the quantification of methylcyclohexane.

Manual integration was employed in deriving certain of the analytical results. The values that have been derived from manual integration are qualified on the quantitation reports. Extracted ion current profiles for each manual integration are included in the data package, and further documented at the end of this submittal. 


\section{DATA REPORTING QUALIFIERS}

Client: Argonne National Laboratory

Job Number: 200-7268-1

Sdg Number: $200-7268$

GC/MS VOA

U

J

$J$

$X$

B
Analyzed for but not detected.

Indicates an Estimated Value for TICs

Indicates an estimated value.

See case narrative notes for explanation of the ' $X$ ' flag

Surrogate exceeds the control limit

The analyte was found in an associated blank, as well as in the sample. 


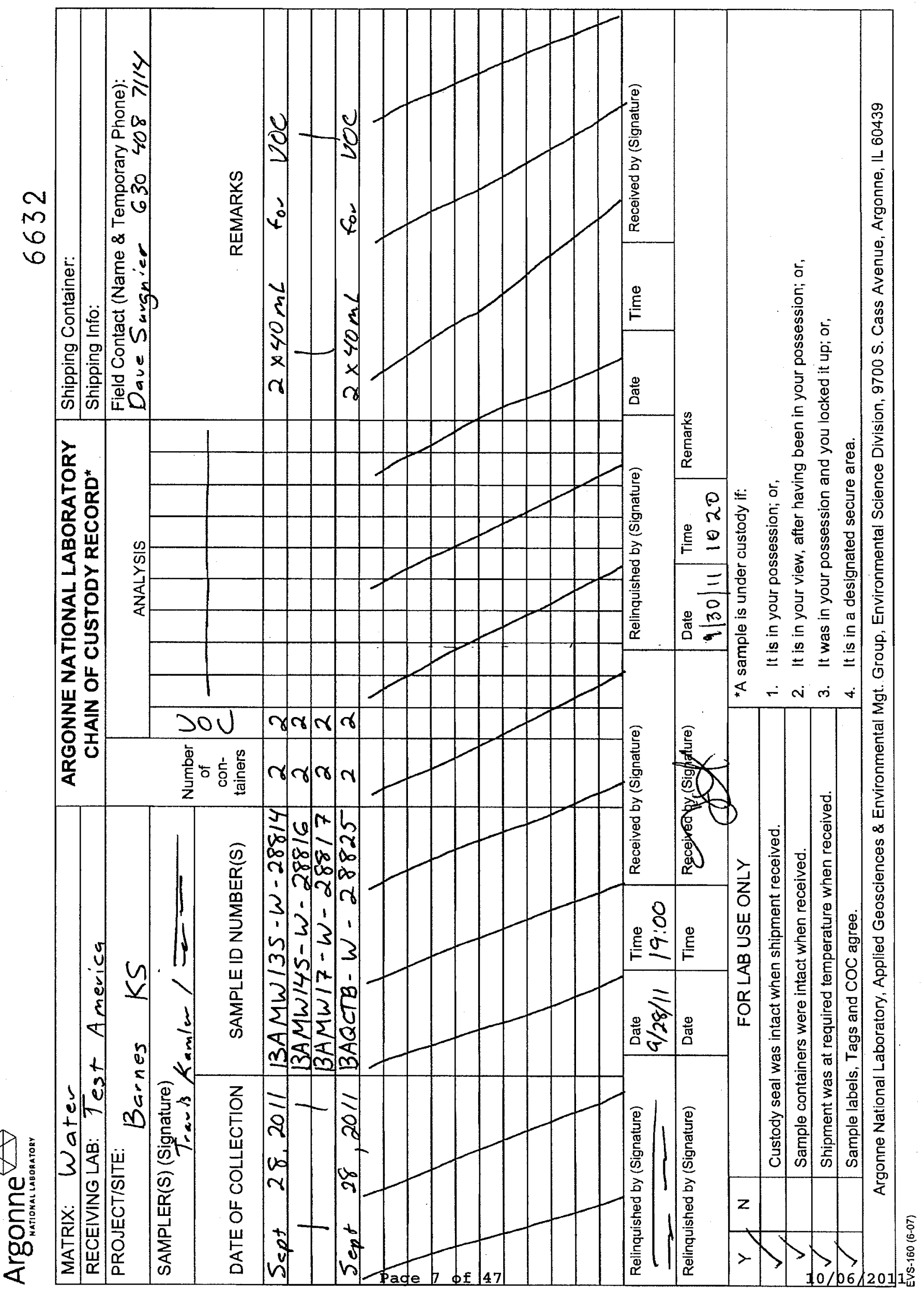




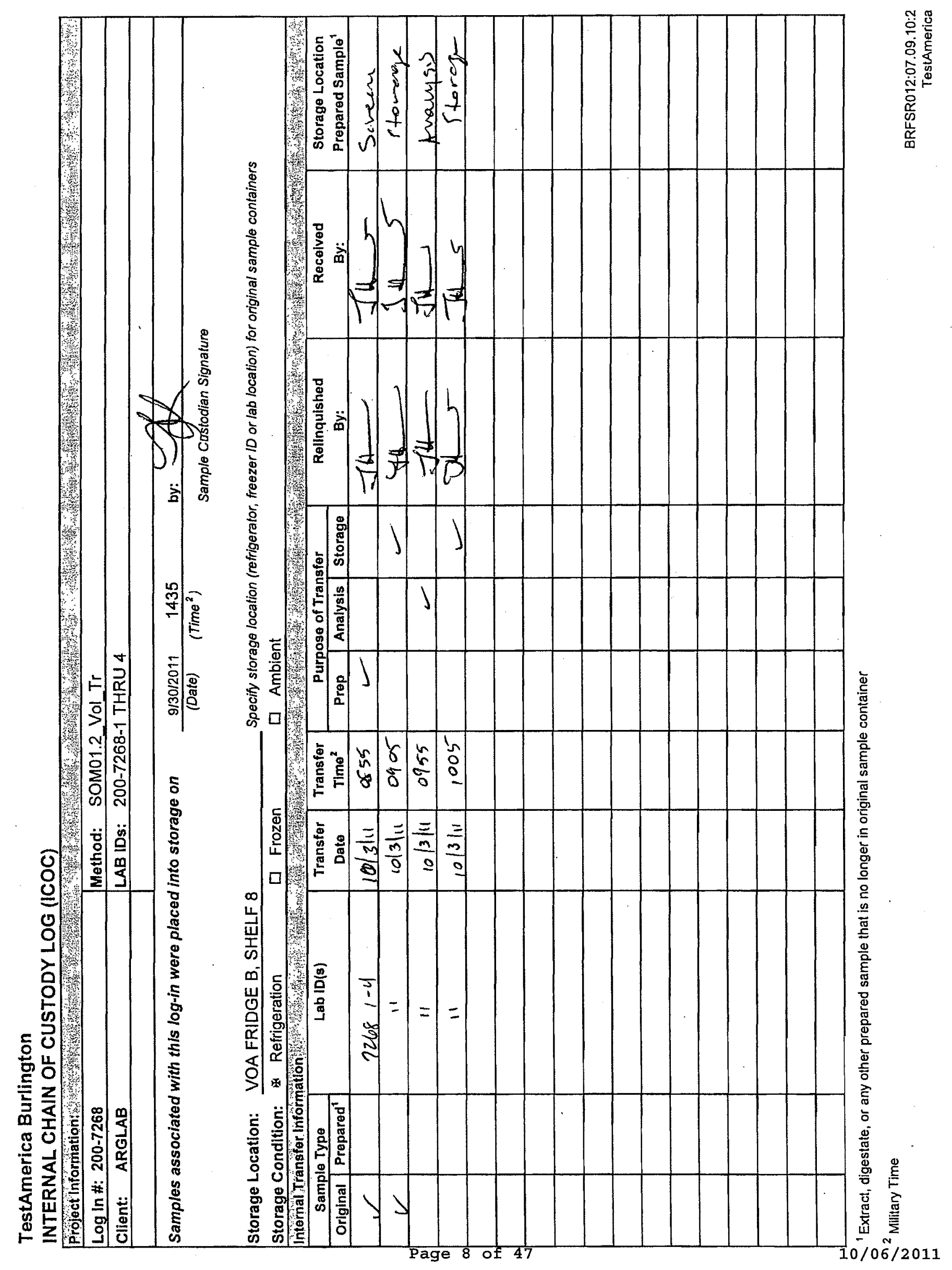




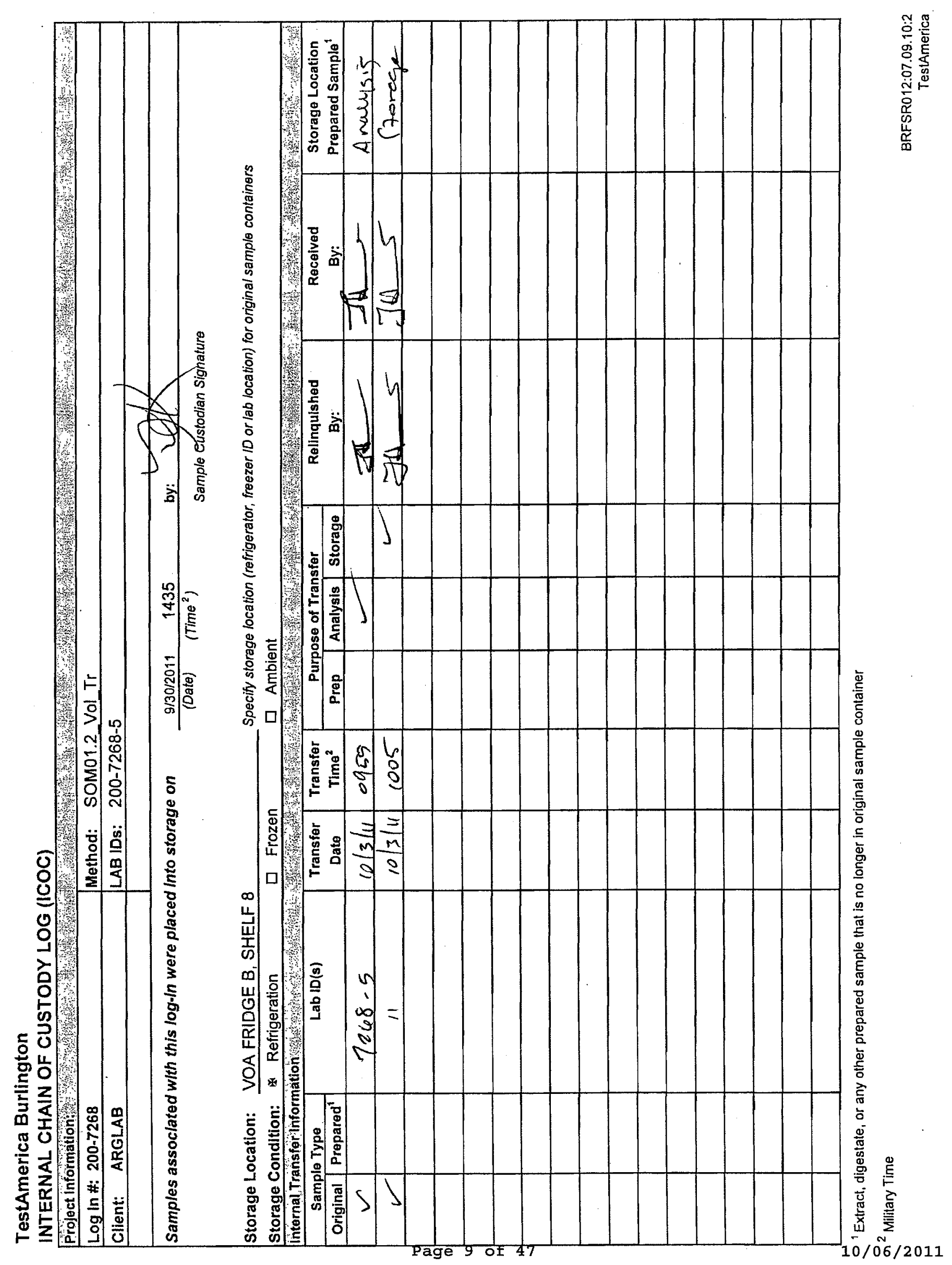




\section{Shipping and Receiving Documents}




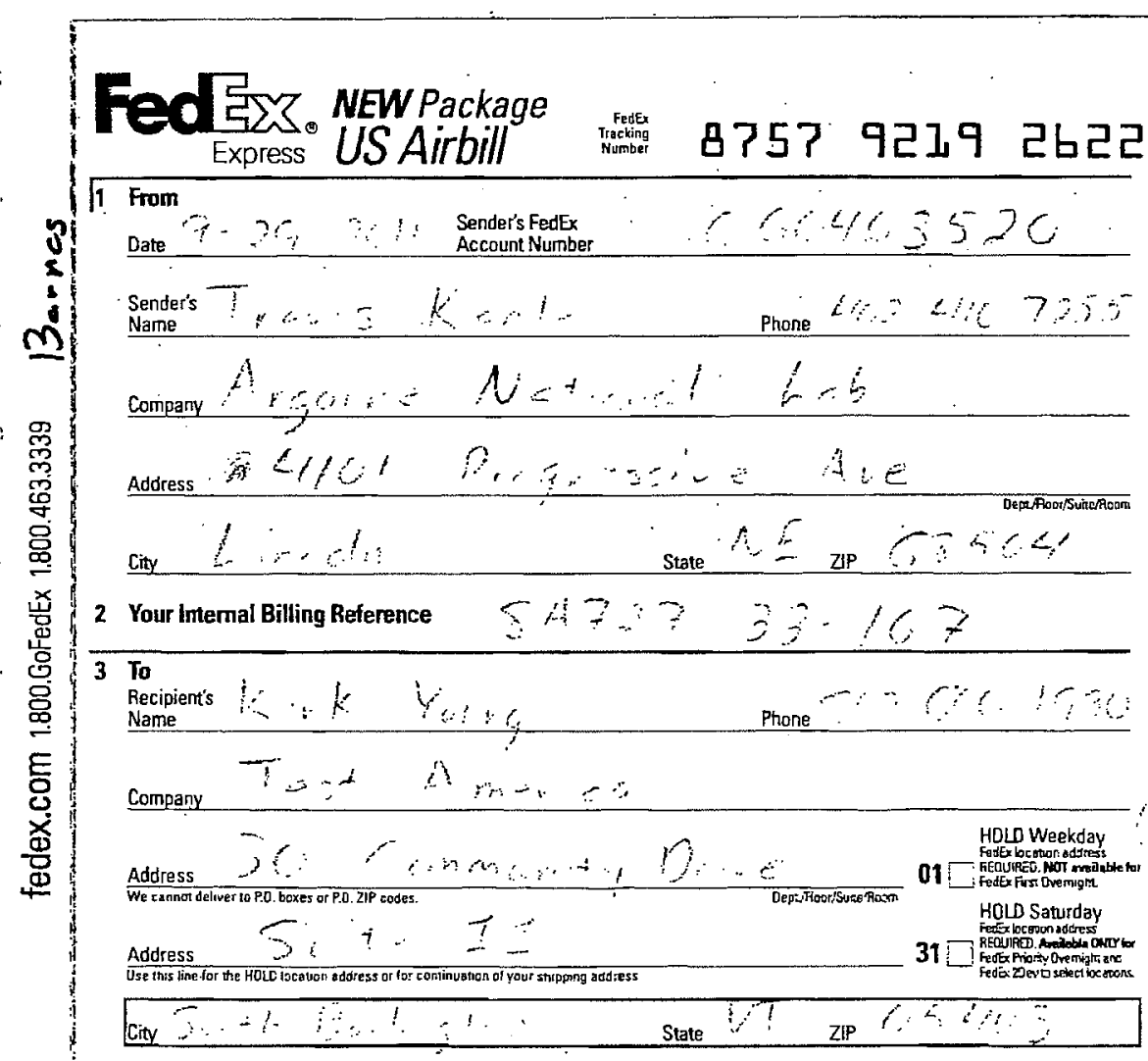

Page 11 of 47
पटD०

FedEx Retrieval

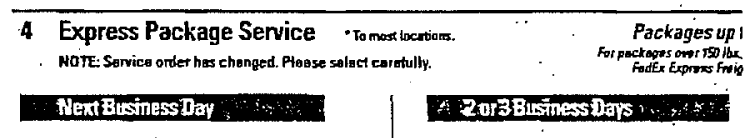

06: EedEx First Qvernight

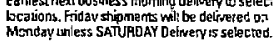

$01-$ FedEx Priority Dvernight

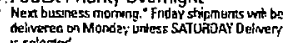
15 selemis

057 FedEx Standard Ovemight

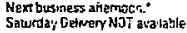

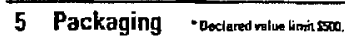

06 FedEx Envelope 02: FedEx Pak $^{*}$ 03 FedEx 04— FedEx $01:$

6 Special Handling and Delivery Signature Options 03: SATURDAY DEUVERY

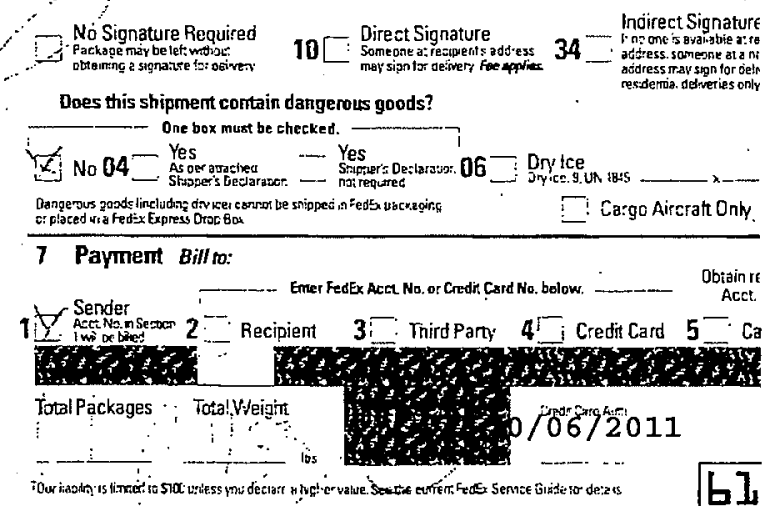




\section{Login Sample Receipt Checklist}

Login Number: 7268

List Source: TestAmerica Burlington

List Number: 1

Creator: Holt, Jamie

Question

$\begin{array}{ll}\text { Answer } & \text { Comment } \\ \text { True } & \text { Lab does not accept radioactive samples. } \\ \text { True } & \text { NO CUSTODY SEAL NUMBERS } \\ \text { True } & \\ \text { True } & \\ \text { True } & \\ \text { True } & \\ \text { True } & \\ \text { True } & \\ \text { True } & \\ \text { True } & \\ \text { True } & \\ \text { True } & \\ \text { True } & \\ \text { True } & \\ \text { True } & \\ \text { True } & \\ \text { True } & \\ \text { N/A } & \\ \text { True } & \\ \text { True } & \\ \text { True } & \\ \text { True } & \\ \text { N/A } & \end{array}$

Radioactivity either was not measured or, if measured, is at or below background

The cooler's custody seal, if present, is intact.

The cooler or samples do not appear to have been compromised or tampered with.

Samples were received on ice.

Cooler Temperature is acceptable.

Cooler Temperature is recorded.

$\mathrm{COC}$ is present.

$\mathrm{COC}$ is filled out in ink and legible.

$\mathrm{COC}$ is filled out with all pertinent information.

is the Field Sampler's name present on COC?

There are no discrepancies between the sample IDs on the containers and the COC.

Samples are received within Holding Time.

Sample containers have legible labels.

Containers are not broken or leaking.

Sample collection date/times are provided.

Appropriate sample containers are used.

Sample bottles are completely filled.

Sample Preservation Verified.

There is sufficient vol. for all requested analyses, incl. any requested MS/MSDs

VOA sample vials do not have headspace or bubble is $<6 \mathrm{~mm}\left(1 / 4^{\prime \prime}\right)$ in diameter.

Multiphasic samples are not present.

Samples do not require splitting or compositing.

Residual Chlorine Checked. 


\section{Sample Login Acknowledgement}

Job 200-7268-1

$\begin{array}{ll}\text { Client Job Description: } & \text { Barnes (200-7268) } \\ \text { Purchase Order \#: } & \text { 1E-30401 } \\ \text { Work Order \#: } & \text { 1E-30401 } \\ \text { Project Manager: } & \text { Kirk F Young } \\ \text { Job Due Date: } & 10 / 14 / 2011 \\ \text { Job TAT: } & 14 \text { Days } \\ \text { Max Deliverable Level: } & \text { IV } \\ \text { Earliest Deliverable Due: } & 10 / 14 / 2011\end{array}$

$\begin{array}{ll}\text { Report To: } & \text { Argonne National Laboratory } \\ & \text { Jorge Alvarado } \\ & 9700 \text { South Cass Avenue } \\ & \text { Building } 203 \\ & \text { Office B-149 } \\ & \text { Argonne, IL } 60439\end{array}$

Bill To: $\quad$ Argonne National Laboratory Accounts Payable Chief Financial Offices 9700 S. Cass Ave. Building 201

Argonne, IL 60439

\section{Login 200-7268}

\begin{tabular}{|c|c|c|c|c|c|}
\hline Sample Receipt: & 9/30/2011 10:20:00 AM & Number of Coolers: & 1 & & \\
\hline Method of Delivery: & FedEx Priority Overnight & Cooler Temperature(s) & $\left(C^{\circ}\right): 0.6$ & & \\
\hline Lab Sample \# & Client Sample ID & Date Sampled & Matrix & & \\
\hline Method & Method Description / Work Location & & & Rpt Basis & Dry $/$ Wet $^{\star \star}$ \\
\hline $200-7268-1$ & BAMW13S-W-28814 & 9/28/2011 12:00:00 AM & Water & & \\
\hline SOM01.2_Vol_Tr & SOM01.2 Trace Volatile Organics / In-Lab & & & Total & Wet \\
\hline $200-7268-2$ & BAMW14S-W-28816 & 9/28/2011 12:00:00 AM & Water & & \\
\hline SOM01.2_Vol_Tr & SOM01.2 Trace Volatile Organics / In-Lab & & & Total & Wet \\
\hline $200-7268-3$ & BAMW17-W-28817 & 9/28/2011 12:00:00 AM & Water & & \\
\hline SOM01.2_Vol_Tr & SOM01.2 Trace Volatile Organics / In-Lab & & & Total & Wet \\
\hline $200-7268-4$ & BAQCTB-W-28825 & 9/28/2011 12:00:00 AM & Water & & \\
\hline SOM01.2_Vol_Tr & SOM01.2 Trace Volatile Organics / In-Lab & & & Total & Wet \\
\hline $200-7268-5$ & VHBLKO1 & 9/30/2011 2:30:00 PM & Water & & \\
\hline SOM01.2_Vol_Tr & SOM01.2 Trace Volatile Organics / In-Lab & & & Total & Wet \\
\hline
\end{tabular}




\section{METHODOLOGY SUMMARY}

Laboratory: TestAmerica Laboratories

Location: South Burlington, Vermont
Project No:

SDG No: $\quad 200-7268$

VOA

Volatile Organics Trace - USEPA CLP SOM01.2 
$2 A$ - FORM II VOA-1

WATER VOLATILE DEUTERATED MONITORING COMPOUND RECOVERY

Lab Name: TESTAMERICA BURLINGTON

Contract: $8 \mathrm{E}-00302$

Lab Code: STLV Case No.: BARNES Mod. Ref No.: SDG NO.: $200-7268$

Level: (TRACE or LOW) TRACE

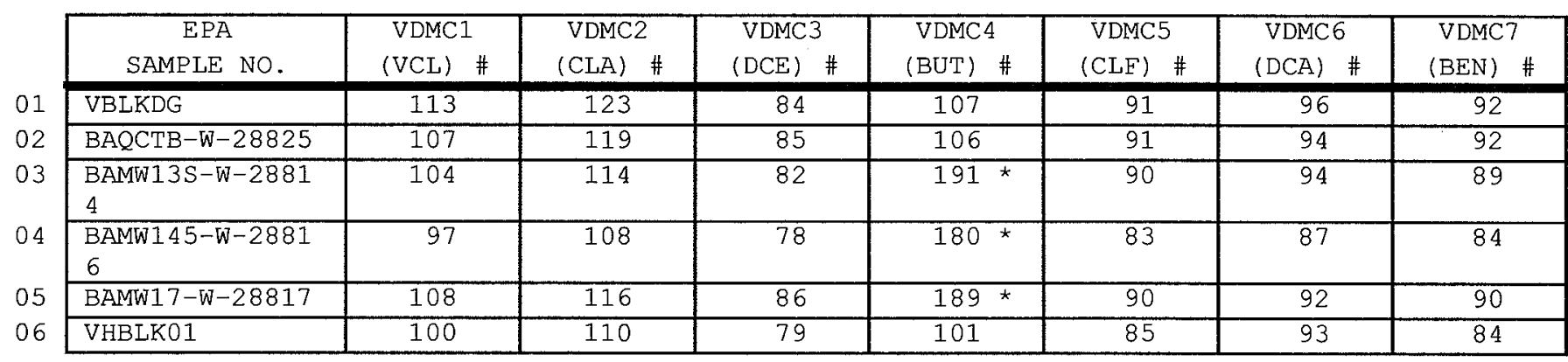

\footnotetext{
VDMC1 (VCL) = Vinyl Chloride-d3

VDMC2 $($ CLA $)=$ Chloroethane-d5

VDMC3 $(\mathrm{DCE})=1,1-\mathrm{Dich})$ loroethene-d2

VDMC4 $(B U T)=2-B u t a n o n e-d 5$

VDMC5 $(\mathrm{CLF})=$ Chloroform-d

VDMC6 $\quad(D C A)=1,2-D i c h l o r o e t h a n e-d 4$

$\operatorname{VDMC7}(\mathrm{BEN})=$ Benzene-d 6
}

$$
\begin{gathered}
\text { QC LIMITS } \\
\hline(65-131) \\
(71-131) \\
(55-104) \\
(49-155) \\
(78-121) \\
(78-129) \\
(77-124)
\end{gathered}
$$

\# Column to be used to flag recovery values

* Values outside of contract required QC limits 
2B - FORM II VOA-2

WATER VOLATILE DEUTERATED MONITORING COMPOUND RECOVERY

Lab Name: TESTAMERICA BURLINGTON

Contract: $8 \mathrm{E}-00302$

Lab Code: STLV Case No.: BARNES Mod. Ref No.: SDG No.: 200-7268

Level: (TRACE or LOW) TRACE

\begin{tabular}{|c|c|c|c|c|c|c|c|c|}
\hline $\begin{array}{c}\text { EPA } \\
\text { SAMPLE NO. }\end{array}$ & $\begin{array}{l}\text { VDMC8 } \\
(\mathrm{DPA}) \#\end{array}$ & $\begin{array}{l}\text { VDMC9 } \\
\text { (TOL) \# }\end{array}$ & $\begin{array}{l}\text { VDMC10 } \\
\text { (TDP) \# }\end{array}$ & $\begin{array}{l}\text { VDMC11 } \\
(\mathrm{HEX}) \#\end{array}$ & $\begin{array}{l}\text { VDMC12 } \\
\text { (TCA) \# }\end{array}$ & $\begin{array}{l}\text { VDMC13 } \\
\text { (DCZ) \# }\end{array}$ & OTHER & $\begin{array}{l}\text { TOT } \\
\text { OUT }\end{array}$ \\
\hline VBLKDG & 96 & 89 & 86 & 93 & 92 & 96 & & 0 \\
\hline BAQCTB-W-28825 & 97 & 89 & 88 & 95 & 94 & 90 & & 0 \\
\hline $\begin{array}{l}\text { BAMW13S-W-2881 } \\
4\end{array}$ & 94 & 87 & 82 & $177 \star$ & 88 & 90 & & 2 \\
\hline $\begin{array}{l}\text { BAMW145-W-2881 } \\
6\end{array}$ & 88 & 82 & 76 & $171 *$ & $\overline{82}$ & 88 & & $\overline{2}$ \\
\hline BAMW17-W-28817 & 93 & 87 & 81 & $174 \star$ & 89 & 93 & & 2 \\
\hline VHBLKO1 & 88 & $\overline{81}$ & 76 & 81 & 85 & 92 & & 0 \\
\hline
\end{tabular}

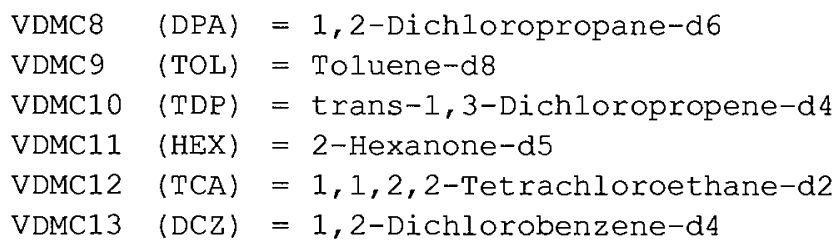

QC LIMITS

$(79-124)$

$(77-121)$

$(73-121)$

$(28-135)$

$(73-125)$

$(80-131)$

\# Column to be used to flag recovery values

* Values outside of contract required QC limits

Report 1,4-Dioxane-d8 for Low-Medium VOA analysis only

Page 1 of 1 


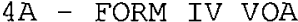

VOLATILE METHOD BLANK SUMMARY
EPA SAMPLE NO.

VBLKDG
Lab Name: TESTAMERICA BURLINGTON

Lab Code: STLV Case No.: BARNES Mod. Ref No.:

Lab File ID: DHSD03.D

Instrument ID: D.i

Matrix: (SOIL/SED/WATER)

Level: (TRACE or LOW/MED)

Water

TRACE

GC Column: DB-624

ID $: 0.20$

$(\mathrm{mm})$
Contract: $8 \mathrm{E}-00302$

SDG No.: 200-7268

Lab Sample ID: MB 200-26126/3

Date Analyzed: 10/03/2011

Time Analyzed: 0919

Heated Purge: ( $Y / N) \quad N$

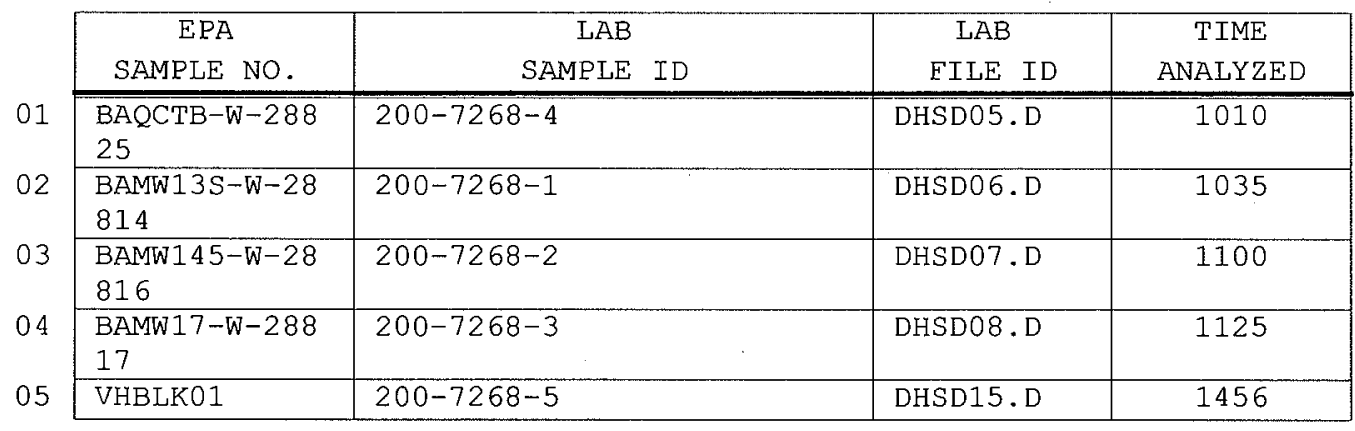

COMMENTS :

Page 1 of 1 
$5 A$ - FORM V VOA

VOLATILE ORGANICS INSTRUMENT

PERFORMANCE CHECK

BROMOELUOROBENZENE (BEB)
EPA SAMPLE NO.

BFBDB

Lab Name: TESTAMERICA BURLINGTON

Contract: $\quad 8 E-00302$

Lab Code: STLV Case No.: BARNES Mod. Ref No.:

SDG No.: 200-7268

Lab File Id: DHS02.D

Instrument Id: D.i

BFB Injection Date: 09/22/2011

BFB Injection Time: 0751

GC Column: $\mathrm{DB}-624$

ID: 0.20 (mm)

\begin{tabular}{|r|l|c|}
\hline $\mathrm{m} / \mathrm{e}$ & ION ABUNDANCE CRITERIA & $\begin{array}{c}\text { RELATIVE } \\
\text { ABUNDANCE }\end{array}$ \\
\hline \hline 50 & $15.0-40.0 \%$ of mass 95 & 20.6 \\
\hline 75 & $30.0-80.0 \%$ of mass 95 & 43.2 \\
\hline 95 & Base peak, 100\% relative abundance & 100 \\
\hline 96 & $5.0-9.0 \%$ of mass 95 & 7.1 \\
\hline 173 & Less than 2.0\% of mass 174 & $0.4 \quad(10.6) 1$ \\
\hline 174 & $50.0-120 \%$ of mass 95 & 71.4 \\
\hline 175 & $5.0-9.0 \%$ of mass 174 & $5.1(1.1) 1$ \\
\hline 176 & $95.0-101 \%$ of mass 174 & $69.8(97.7) 1$ \\
\hline 177 & $5.0-9.0 \%$ of mass 176 & 4.5( \\
\hline
\end{tabular}

1 - Value is omass 174

2 - Value is omass 176

\begin{tabular}{c|c|c|c|c|c|}
\cline { 2 - 7 } & \multicolumn{1}{|c|}{$\begin{array}{c}\text { EPA } \\
\text { SAMPLE NO. }\end{array}$} & \multicolumn{1}{c|}{ LAB } & DATE & TIME \\
SAMPLE ID & FILE ID & ANALYZED & ANALYZED \\
\cline { 2 - 7 } 01 & VSTD0.5DB & IC 200-25802/5 & DHS05.D & $09 / 22 / 2011$ & 0848 \\
\cline { 2 - 7 } 03 & VSTD001DB & IC 200-25802/6 & DHS06.D & $09 / 22 / 2011$ & 0913 \\
\cline { 2 - 7 } 04 & VSTD005DB & ICIS 200-25802/7 & DHS07.D & $09 / 22 / 2011$ & 0938 \\
\cline { 2 - 7 } & VSTD010DB & IC 200-25802/8 & DHS08.D & $09 / 22 / 2011$ & 1002 \\
\cline { 2 - 7 } & VSTD020DB & IC 200-25802/9 & DHS09.D & $09 / 22 / 2011$ & 1038 \\
\cline { 2 - 7 }
\end{tabular}


$5 A$ - FORM V VOA

VOLATILE ORGANICS INSTRUMENT

PEREORMANCE CHECK

BROMOFLUOROBENZENE (BEB)
EPA SAMPLE NO.

BEBDG
Lab Name: TESTAMERICA BURLINGTON

Lab Code: STLV Case No.

Lab.File Id: DHSD01.D

Instrument Id: D.i

GC Column: DB-624

ID $: 0.20$
Contract: $8 \mathrm{E}-00302$

SDG No.: $200-7268$

BFB Injection Date: 10/03/2011

BEB Injection Time: 0835 $(\mathrm{mm})$

\begin{tabular}{|c|c|c|}
\hline $\mathrm{m} / \mathrm{e}$ & ION ABUNDANCE CRITERIA & $\begin{array}{l}\text { \% RELATIVE } \\
\text { ABUNDANCE }\end{array}$ \\
\hline 50 & $15.0-40.0 \%$ of mass 95 & 25.7 \\
\hline 75 & $30.0-80.0 \%$ of mass 95 & 42.9 \\
\hline 95 & Base peak, $100 \%$ relative abundance & 100 \\
\hline 96 & $5.0-9.0 \%$ of mass 95 & 6.8 \\
\hline 173 & Less than $2.0 \%$ of mass 174 & 0.41 \\
\hline 174 & $50.0-120 \%$ of mass 95 & 82.2 \\
\hline 175 & $5.0-9.0 \%$ of mass 174 & $5.5(6.7) 1$ \\
\hline 176 & $95.0-101 \%$ of mass 174 & $78.2(95.2) 1$ \\
\hline 177 & $5.0-9.0 \%$ of mass 176 & $4.7(6.0) 2$ \\
\hline
\end{tabular}

1 - va1ue is omass 174

2 - Value is omass 176

\begin{tabular}{|c|c|c|c|c|}
\hline $\begin{array}{c}\text { EPA } \\
\text { SAMPLE NO. }\end{array}$ & $\begin{array}{r}\text { LAB } \\
\text { SAMPLE ID }\end{array}$ & $\begin{array}{l}\text { LAB } \\
\text { FILE ID }\end{array}$ & $\begin{array}{c}\text { DATE } \\
\text { ANALYZED }\end{array}$ & $\begin{array}{c}\text { TIME } \\
\text { ANALYZED }\end{array}$ \\
\hline VSTD005DG & CCVIS $200-26126 / 2$ & DHSD02.D & $10 / 03 / 2011$ & 0854 \\
\hline VBLKDG & MB $200-26126 / 3$ & DHSD03.D & $10 / 03 / 2011$ & 0919 \\
\hline $\begin{array}{l}\text { BAQCTB-W-2 } \\
8825\end{array}$ & $200-7268-4$ & DHSD0 5.D & $10 / 03 / 2011$ & 1010 \\
\hline $\begin{array}{l}\text { BAMW13S-W- } \\
28814\end{array}$ & $200-7268-1$ & DHSD0 6.D & $10 / 03 / 2011$ & 1035 \\
\hline $\begin{array}{l}\text { BAMW145-W- } \\
28816\end{array}$ & $200-7268-2$ & DHSD0 7. D & $10 / 03 / 2011$ & 1100 \\
\hline $\begin{array}{l}\text { BAMW 17-W-2 } \\
8817\end{array}$ & $200-7268-3$ & DHSD08.D & $10 / 03 / 2011$ & 1125 \\
\hline VHBLKO1 & $200-7268-5$ & DHSD15. D & $10 / 03 / 2011$ & 1456 \\
\hline VSTD005GD & CCVC $200-26126 / 16$ & DHSD16.D & $10 / 03 / 2011$ & 1521 \\
\hline
\end{tabular}


$8 A$ - FORM VIII VOA

VOLATILE INTERNAL STANDARD AREA AND RETENTION TIME SUMMARY

Lab Name: TESTAMERICA BURLINGTON

Contract: $8 \mathrm{E}-00302$

Lab Code: STLV Case No.: BARNES Mod. Ref No.: SDG No.: $200-7268$

GC Column: DB-624 ID $: 0.20$ (mm) Init. Calib. Date(s)

$09 / 22 / 201109 / 22 / 2011$

EPA Sample No. (VSTD\#\#\#\#) : VSTD005DG

Date Analyzed: 10/03/2011

Lab File ID (Standard): DHSD02.D

Time Analyzed: 0854

Instrument ID: D.i

Heated Purge: $(\mathrm{Y} / \mathrm{N}) \mathrm{N}$

\begin{tabular}{|c|c|c|c|c|c|c|}
\hline & $\begin{array}{c}\text { IS1 (CBZ) } \\
\text { AREA }\end{array}$ & RT \# & $\begin{array}{c}\text { IS2 (DFB) } \\
\text { AREA }\end{array}$ & $\mathrm{RT} \quad \#$ & $\begin{array}{c}\text { IS3 (DCB) } \\
\text { AREA }\end{array}$ & RT \\
\hline 12 HOUR STD & 203303 & 8.73 & 239176 & 5.37 & 100305 & 11.56 \\
\hline UPPER LIMIT & 284624 & 9.06 & 334846 & 5.70 & 140427 & 11.89 \\
\hline LOWER LIMIT & 121982 & 8.40 & 143506 & 5.04 & 60183 & 11.23 \\
\hline EPA SAMPLE NO. & & & & & & \\
\hline$\overline{\text { VBLKDG }}$ & 192667 & 8.73 & 223517 & 5.37 & 82863 & 11.56 \\
\hline BAQCTB-W-28825 & 175366 & 8.73 & 204518 & 5.37 & 78960 & 11.56 \\
\hline $\begin{array}{l}\text { BAMW13S-W-2881 } \\
4\end{array}$ & 174948 & 8.73 & 205714 & 5.37 & 72759 & 11.56 \\
\hline $\begin{array}{l}\text { BAMW145-W-2881 } \\
6\end{array}$ & 184082 & 8.73 & 216076 & 5.37 & 75905 & 11.56 \\
\hline BAMW17-W-28817 & 177767 & 8.73 & 204092 & 5.37 & 72714 & 11.56 \\
\hline VHBLK01 & 163937 & 8.73 & 184959 & 5.37 & 66069 & 11.56 \\
\hline
\end{tabular}

IS1 $(\mathrm{CBZ})=$ Chlorobenzene $-\mathrm{d} 5$

IS2 $(\mathrm{DFB})=1,4$-Difluorobenzene

IS3 $(D C B)=1,4-$ Dichlorobenzene-d4

AREA UPPER LIMIT $=140 \%$ (Trace Volatiles) of internal standard area

AREA LOWER LIMIT $=60 \%$ (Trace Volatiles) of internal standard area

RT UPPER LIMIT $=+0.33$ (Trace Volatiles) minutes of internal standard RT

RT LOWER LIMIT $=-0.33$ (Trace Volatiles) minutes of internal standard RT

\# Column used to flag values outside contract required QC limits with an asterisk.

Page 1 of 1 
$1 A$ - FORM I VOA-1

VOLATILE ORGANICS ANALYSIS DATA SHEET
EPA SAMPLE NO

BAMW $13 S-W-28814$
Lab Name: TESTAMERICA BURLINGTON

Lab Code: STLV

Matrix: (SOIL/SED/WATER)

Sample wt/vol: 25.0

Case No.: BARNES

Mor

Mod. Ref No.:

Water

$(\mathrm{g} / \mathrm{mL}) \mathrm{mL}$

Contract: $8 \mathrm{E}-00302$

Leve1: (TRACE/LOW/MED) TRACE

\% Moisture: not dec.

GC Column: DB-624 ID : $0.20 \quad(\mathrm{~mm})$

Soil Extract Volume: (uL) (mL)

Purge Volume: 25.0
SDG NO.: 200-7268

Lab Sample ID: 200-7268-1

Lab File ID: DHSD06.D

Date Received: 09/30/2011

Date Analyzed: 10/03/2011

Dilution Factor: 1.0

Soil Aliquot Volume:

(uL)

\begin{tabular}{|c|c|c|c|}
\hline CAS NO. & COMPOUND & $\begin{array}{l}\text { CONCENTRATION UNITS: } \\
(\mathrm{ug} / \mathrm{L} \text { or } \mathrm{ug} / \mathrm{kg}) \mathrm{ug} / \mathrm{L}\end{array}$ & $Q$ \\
\hline $75-71-8$ & Dichlorodifluoromethane & 0.50 & $\mathrm{U}$ \\
\hline $74-87-3$ & Chloromethane & 0.50 & $\mathrm{U}$ \\
\hline $75-01-4$ & Vinyl chloride & 0.50 & $\mathrm{U}$ \\
\hline $74-83-9$ & Bromomethane & 0.50 & $\mathrm{U}$ \\
\hline $75-00-3$ & Chloroethane & 0.50 & $\mathrm{U}$ \\
\hline $75-69-4$ & Trichlorofluoromethane & 0.50 & $\mathrm{U}$ \\
\hline $75-35-4$ & 1,1-Dichloroethene & 0.50 & $\mathrm{U}$ \\
\hline $76-13-1$ & 1, 1,2-Trichloro-1,2,2-trifluoroethane & 0.50 & $\mathrm{U}$ \\
\hline $67-64-1$ & Acetone & 1.9 & $\mathrm{JB}$ \\
\hline $75-15-0$ & Carbon disulfide & 0.50 & $\mathrm{U}$ \\
\hline $79-20-9$ & Methyl acetate & 0.50 & $\mathrm{U}$ \\
\hline $75-09-2$ & Methylene Chloride & 0.50 & $\mathrm{U}$ \\
\hline $156-60-5$ & trans-1,2-Dichloroethene & 0.50 & $\mathrm{U}$ \\
\hline $1634-04-4$ & Methyl tert-butyl ether & 0.50 & $\mathrm{U}$ \\
\hline $75-34-3$ & 1,1-Dichloroethane & 0.50 & $\mathrm{U}$ \\
\hline $156-59-2$ & cis-1,2-Dichloroethene & 0.50 & $\mathrm{U}$ \\
\hline $78-93-3$ & 2-Butanone & 5.0 & $\mathrm{U}$ \\
\hline $74-97-5$ & Bromochloromethane & 0.50 & $\mathrm{U}$ \\
\hline $67-66-3$ & Chloroform & 0.69 & \\
\hline $71-55-6$ & $1,1,1$-Trichloroethane & 0.50 & $\mathrm{U}$ \\
\hline $110-82-7$ & Cyclohexane & 0.50 & U \\
\hline $56-23-5$ & Carbon tetrachloride & 8.3 & \\
\hline $71-43-2$ & Benzene & 0.50 & $\mathrm{U}$ \\
\hline $107-06-2$ & 1,2-Dichloroethane & 0.50 & $\mathrm{U}$ \\
\hline
\end{tabular}


$1 B$ - FORM I VOA-2

VOLATILE ORGANICS ANALYSIS DATA SHEET
EPA SAMPLE NO.

BAMW1 3S-W-28814
Lab Name: TESTAMERICA BURLINGTON

Lab Code: STLV Ca
Matrix: (SOIL/SED/WATER)

Sample wt/vol: 25.0

Level: (TRACE/LOW/MED)

: Moisture: not dec.

GC Column: DB-624 ID: $0.20 \quad(\mathrm{~mm})$

Soil Extract Volume:

Purge Volume: 25.0 (mL)
Contract: $8 \mathrm{E}-00302$ SDG No.: 200-7268

Lab Sample ID: 200-7268-1

Lab File ID: DHSDO6.D

Date Received: 09/30/2011

Date Analyzed: 10/03/2011

Dilution Factor: 1.0

Soil Aliquot Volume: (uL)

\begin{tabular}{|c|c|c|c|}
\hline CAS NO. & COMPOUND & $\begin{array}{l}\text { CONCENTRATION UNITS: } \\
(\mathrm{ug} / \mathrm{L} \text { or } \mathrm{ug} / \mathrm{kg}) \mathrm{ug} / \mathrm{L}\end{array}$ & $Q$ \\
\hline $79-01-6$ & Trichloroethene & 0.50 & $\overline{\mathrm{U}}$ \\
\hline $108-87-2$ & Methylcyclohexane & 0.50 & U \\
\hline $78-87-5$ & 1,2-Dichloropropane & 0.50 & $\mathrm{U}$ \\
\hline $75-27-4$ & Bromodichloromethane & 0.50 & $\mathrm{U}$ \\
\hline $10061-01-5$ & cis-1,3-Dichloropropene & 0.50 & $\mathrm{U}$ \\
\hline $108-10-1$ & 4-Methyl-2-pentanone & 5.0 & $\mathrm{~J}$ \\
\hline $108-88-3$ & Toluene & 0.026 & $\mathrm{~J} B$ \\
\hline $10061-02-6$ & trans $-1,3-$ Dichloropropene & 0.50 & $\mathrm{~J}$ \\
\hline $79-00-5$ & $1,1,2$-Trichloroethane & 0.50 & $\mathrm{~J}$ \\
\hline $127-18-4$ & Tetrachloroethene & 0.50 & $\mathrm{U}$ \\
\hline $591-78-6$ & 2-Hexanone & 5.0 & $\mathrm{U}$ \\
\hline $124-48-1$ & Dibromochloromethane & 0.50 & $\mathrm{U}$ \\
\hline $106-93-4$ & 1,2-Dibromoethane & 0.50 & $\bar{U}$ \\
\hline $108-90-7$ & Chlorobenzene & 0.50 & $\mathrm{U}$ \\
\hline $100-41-4$ & Ethylbenzene & 0.50 & $\mathrm{U}$ \\
\hline $95-47-6$ & o-Xylene & 0.50 & $\mathrm{U}$ \\
\hline $179601-23-1$ & $\mathrm{~m}, \mathrm{p}-\mathrm{Xylene}$ & 0.50 & $\mathrm{U}$ \\
\hline $100-42-5$ & Styrene & 0.50 & $\mathrm{U}$ \\
\hline $75-25-2$ & Bromoform & 0.50 & U \\
\hline $98-82-8$ & Isopropylbenzene & 0.50 & $\mathrm{U}$ \\
\hline $79-34-5$ & $1,1,2,2$-Tetrachloroethane & 0.50 & $\mathrm{U}$ \\
\hline $541-73-1$ & 1,3-Dichlorobenzene & 0.50 & $\mathrm{U}$ \\
\hline $106-46-7$ & 1,4-Dichlorobenzene & 0.50 & $\mathrm{U}$ \\
\hline $95-50-1$ & 1,2-Dichlorobenzene & 0.50 & $\mathrm{U}$ \\
\hline $96-12-8$ & 1,2-Dibromo-3-Chloropropane & 0.50 & U \\
\hline $120-82-1$ & $1,2,4$-Trichlorobenzene & 0.50 & U \\
\hline $87-61-6$ & 1,2,3-Trichlorobenzene & 0.50 & U \\
\hline
\end{tabular}


$1 \mathrm{~J}$ - FORM I VOA-TIC

VOLATILE ORGANICS ANALYSIS DATA SHEET TENTATIVELY IDENTIFIED COMPOUNDS
EPA SAMPLE NO.

BAMW13S-W-28814

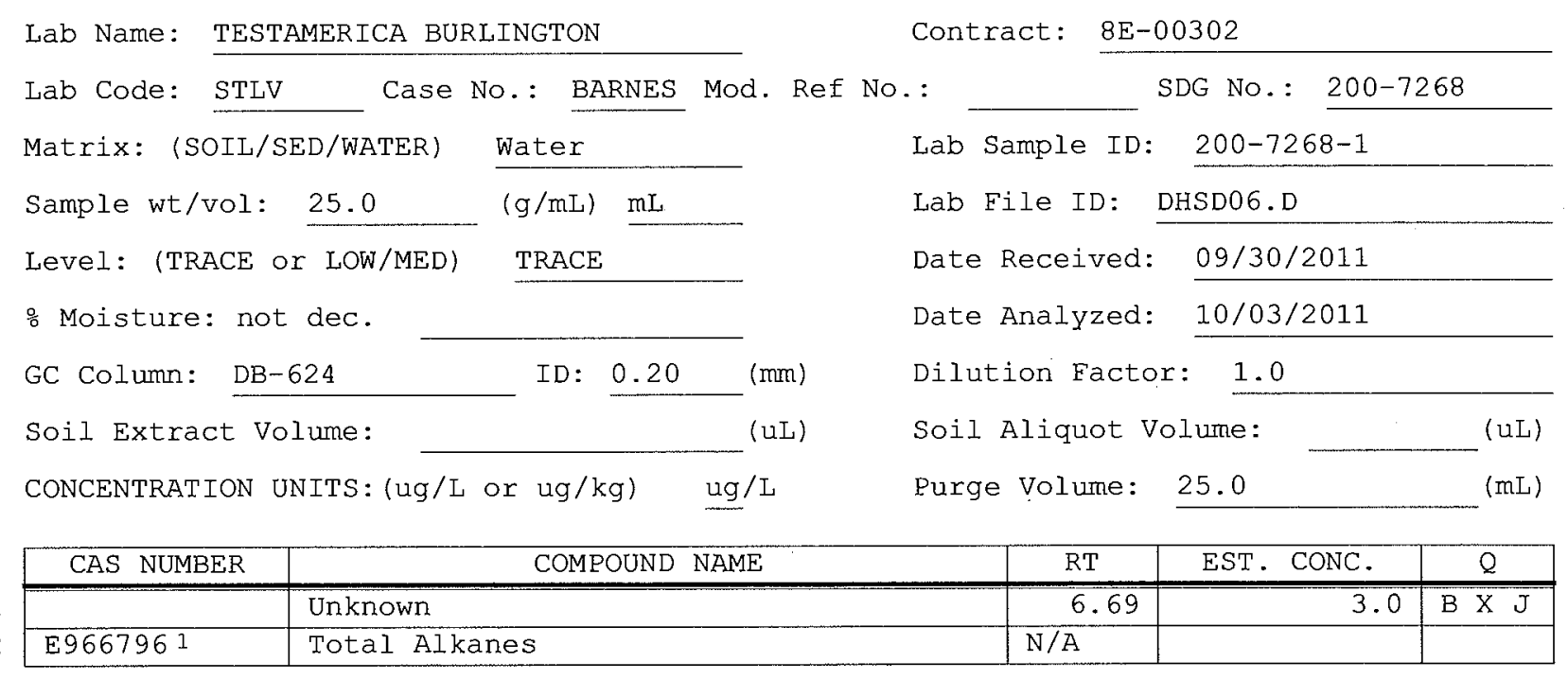

1 EPA-designated Registry Number. 
Lab Name: TESTAMERICA BURLINGTON

Lab Code: STLV Case No.: BARNES Mod. Ref No.:
Contract: $8 \mathrm{E}-00302$

SDG No.: 200-7268
Matrix: (SOIL/SED/WATER) water

Sample wt/vol: 25.0 $(\mathrm{g} / \mathrm{mL}) \mathrm{mL}$

Level: (TRACE/LOW/MED) TRACE

o Moisture: not dec.

GC Column: DB-624 ID $: 0.20 \quad(\mathrm{~mm})$

Soil Extract Volume: (uL) (mL)

Purge Volume: 25.0
Lab Sample ID: 200-7268-2

Lab File ID: DHSD07.D

Date Received: 09/30/2011

Date Analyzed: 10/03/2011

Dilution Factor: 1.0

Soil Aliquot Volume:

(uL)

\begin{tabular}{|c|c|c|c|}
\hline CAS NO. & COMPOUND & $\begin{array}{l}\text { CONCENTRATION UNITS: } \\
(\mathrm{ug} / \mathrm{L} \text { or } \mathrm{ug} / \mathrm{kg}) \mathrm{ug} / \mathrm{L}\end{array}$ & $Q$ \\
\hline $75-71-8$ & Dichlorodifluoromethane & 0.50 & $\overline{\mathrm{U}}$ \\
\hline $74-87-3$ & Chloromethane & 0.50 & $\mathrm{U}$ \\
\hline $75-01-4$ & Vinyl chloride & 0.50 & $\mathrm{U}$ \\
\hline $74-83-9$ & Bromomethane & 0.50 & $\mathrm{U}$ \\
\hline $75-00-3$ & Chloroethane & 0.50 & $\mathrm{U}$ \\
\hline $75-69-4$ & Trichlorofluoromethane & 0.50 & $\mathrm{U}$ \\
\hline $75-35-4$ & 1,1-Dichloroethene & 0.50 & $\mathrm{U}$ \\
\hline $76-13-1$ & $1,1,2$-Trichloro-1,2,2-trifluoroethane & 0.50 & $\mathrm{U}$ \\
\hline $67-64-1$ & Acetone & 5.0 & $\mathrm{U}$ \\
\hline $75-15-0$ & Carbon disulfide & 0.50 & $\mathrm{U}$ \\
\hline $79-20-9$ & Methyl acetate & 0.50 & $\mathrm{U}$ \\
\hline $75-09-2$ & Methylene Chloride & 0.50 & $\mathrm{U}$ \\
\hline $156-60-5$ & trans-1,2-Dichloroethene & 0.50 & $\mathrm{U}$ \\
\hline $1634-04-4$ & Methyl tert-butyl ether & 0.50 & $\mathrm{U}$ \\
\hline $75-34-3$ & 1,1-Dichloroethane & 0.50 & $\mathrm{U}$ \\
\hline $156-59-2$ & cis-1,2-Dichloroethene & 0.50 & $\mathrm{U}$ \\
\hline $78-93-3$ & 2-Butanone & 5.0 & $\mathrm{U}$ \\
\hline $74-97-5$ & Bromochlorome thane & 0.50 & $\mathrm{U}$ \\
\hline $67-66-3$ & Chloroform & 0.21 & $\mathrm{~J}$ \\
\hline $71-55-6$ & 1,1,1-Trichloroethane & 0.50 & $\mathrm{U}$ \\
\hline $110-82-7$ & Cyclohexane & 0.50 & $\mathrm{U}$ \\
\hline $56-23-5$ & Carbon tetrachloride & 2.8 & \\
\hline $71-43-2$ & Benzene & 0.50 & $\mathrm{U}$ \\
\hline $107-06-2$ & 1,2-Dichloroethane & 0.50 & $\mathrm{U}$ \\
\hline
\end{tabular}

Report 1,4-Dioxane for Low-Medium VOA analysis only 
$1 B$ - FORM I VOA-2

VOLATILE ORGANICS ANALYSIS DATA SHEET
EPA SAMPLE NO.

BAMW1 45-W-28816
Lab Name: TESTAMERICA BURLINGTON

Lab Code: STLV Case No.: BARNES Mod. Ref No.:
Contract: $\quad 8 \mathrm{E}-00302$

SDG No.: $200-7268$
Matrix: (SOIL/SED/WATER) Water

Sample wt/vol: 25.0

$(\mathrm{g} / \mathrm{mL}) \mathrm{mL}$

Level: (TRACE/LOW/MED) TRACE

\% Moisture: not dec.

GC Column: DB-624 ID : $0.20 \quad(\mathrm{~mm})$

Soil Extract Volume: (uL) (mL)

Purge Volume: 25.0
Lab Sample ID: 200-7268-2

Lab File ID: DHSD07.D

Date Received: 09/30/2011

Date Analyzed: 10/03/2011

Dilution Factor: 1.0

Soil Aliquot Volume:

(uL)

\begin{tabular}{|c|c|c|c|}
\hline CAS NO. & COMPOUND & $\begin{array}{l}\text { CONCENTRATION UNITS: } \\
(\mathrm{ug} / \mathrm{L} \text { or } \mathrm{ug} / \mathrm{kg}) \mathrm{ug} / \mathrm{L}\end{array}$ & $\mathrm{Q}$ \\
\hline $79-01-6$ & Trichloroethene & 0.50 & $\overline{\mathrm{U}}$ \\
\hline $108-87-2$ & Methylcyclohexane & 0.50 & $\mathrm{U}$ \\
\hline $78-87-5$ & 1,2-Dichloropropane & 0.50 & $\mathrm{U}$ \\
\hline $75-27-4$ & Bromodichloromethane & 0.50 & $\mathrm{U}$ \\
\hline $10061-01-5$ & cis-1,3-Dichloropropene & 0.50 & $\mathrm{U}$ \\
\hline $108-10-1$ & 4-Methyl-2-pentanone & 5.0 & $\mathrm{U}$ \\
\hline $108-88-3$ & Toluene & 0.0071 & $\mathrm{JB}$ \\
\hline $10061-02-6$ & trans-1,3-Dichloropropene & 0.50 & $\mathrm{U}$ \\
\hline $79-00-5$ & 1,1,2-Trichloroethane & 0.50 & $\mathrm{U}$ \\
\hline $127-18-4$ & Tetrachloroethene & 0.50 & $\mathrm{U}$ \\
\hline $591-78-6$ & 2-Hexanone & 5.0 & $\mathrm{U}$ \\
\hline $124-48-1$ & Dibromochloromethane & 0.50 & $\mathrm{U}$ \\
\hline $106-93-4$ & 1,2-Dibromoethane & 0.50 & $\mathrm{U}$ \\
\hline $108-90-7$ & Chlorobenzene & 0.50 & $\mathrm{U}$ \\
\hline $100-41-4$ & Ethylbenzene & 0.50 & $\mathrm{U}$ \\
\hline $95-47-6$ & o-xylene & 0.50 & $\mathrm{U}$ \\
\hline $179601-23-1$ & $\mathrm{~m}, \mathrm{p}$-Xylene & 0.50 & $\mathrm{U}$ \\
\hline $100-42-5$ & styrene & 0.50 & $\mathrm{U}$ \\
\hline $75-25-2$ & Bromoform & 0.50 & $\mathrm{U}$ \\
\hline $98-82-8$ & Isopropylbenzene & 0.50 & $\mathrm{U}$ \\
\hline $79-34-5$ & $1,1,2,2$-Tetrachloroethane & 0.50 & $\mathrm{U}$ \\
\hline $541-73-1$ & 1,3-Dichlorobenzene & 0.50 & $\mathrm{U}$ \\
\hline $106-46-7$ & 1,4-Dichlorobenzene & 0.50 & $\mathrm{U}$ \\
\hline $95-50-1$ & 1,2-Dichlorobenzene & 0.50 & $\mathrm{U}$ \\
\hline $96-12-8$ & 1,2-Dibromo-3-Chloropropane & 0.50 & $\mathrm{U}$ \\
\hline $120-82-1$ & 1,2,4-Trichlorobenzene & 0.50 & $\mathrm{U}$ \\
\hline $87-61-6$ & 1,2,3-Trichlorobenzene & 0.50 & $\mathrm{U}$ \\
\hline
\end{tabular}


$1 \mathrm{~J}-$ FORM I VOA-TIC

VOLATILE ORGANICS ANALYSIS DATA SHEET TENTATIVELY IDENTIEIED COMPOUNDS
EPA SAMPLE NO.

BAMW $145-W-28816$
Lab Name: TESTAMERICA BURLINGTON

Lab Code: STLV Case No.

Matrix: (SOIL/SED/WATER)

Water

Sample wt/vol: 25.0 $(\mathrm{g} / \mathrm{mL}) \quad \mathrm{mL}$

Level: (TRACE or LOW/MED)

TRACE

음 Moisture: not dec.

GC Column: DB-624 ID $: 0.20$ (mm)

Soil Extract Volume: (uL)

CONCENTRATION UNITS: (ug/L or $\mathrm{ug} / \mathrm{kg}$ ) $\mathrm{ug} / \mathrm{L}$
Contract: $\quad 8 \mathrm{E}-00302$

SDG No.: 200-7268

Lab Sample ID: 200-7268-2

Lab File ID: DHSD07.D

Date Received: 09/30/2011

Date Analyzed: 10/03/2011

Dilution Factor: 1.0

Soil Aliquot Volume: (UL)

Purge Volume: 25.0 (mL)

01

\begin{tabular}{|l|l|c|c|c|}
\hline CAS NUMBER & \multicolumn{1}{|c|}{ COMPOUND NAME } & RT & EST. CONC. & Q \\
\hline \hline & Unknown & 6.69 & 2.8 & $B$ X J \\
\hline E9667961 & Total Alkanes & N/A & & \\
\hline
\end{tabular}

\footnotetext{
1 EPA-designated Registry Number.
} 
$1 A$ - FORM I VOA-1

VOLATILE ORGANICS ANALYSIS DATA SHEET
EPA SAMPLE NO.

BAMW1 7-W-28817
Lab Name: TESTAMERICA BURLINGTON

Lab Code: STLV Case

Matrix: (SOIL/SED/WATER)

\section{Water}

$(\mathrm{g} / \mathrm{mL}) \mathrm{mL}$

Sample wt/vol: 25.0

TRACE

Level: (TRACE/LOW/MED)

o Moisture: not dec.

GC Column: DB-624 ID $: 0.20 \quad$ (mm)

Soil Extract Volume:

Purge Volume: 25.0
Contract: $8 \mathrm{E}-00302$

SDG No.: 200-7268

Lab Sample ID: 200-7268-3

Lab File ID: DHSD08.D

Date Received: 09/30/2011

Date Analyzed: 10/03/2011

Dilution Factor: 1.0

Soil Aliquot Volume:

(uL)

\begin{tabular}{|c|c|c|c|}
\hline CAS NO. & COMPOUND & $\begin{array}{l}\text { CONCENTRATION UNITS: } \\
(\mathrm{ug} / \mathrm{L} \text { or } \mathrm{ug} / \mathrm{kg}) \mathrm{ug} / \mathrm{L}\end{array}$ & Q \\
\hline $75-71-8$ & Dichlorodifluoromethane & 0.50 & $\mathrm{U}$ \\
\hline $74-87-3$ & Chloromethane & 0.50 & $\mathrm{U}$ \\
\hline $75-01-4$ & Vinyl chloride & 0.50 & $\mathrm{U}$ \\
\hline $74-83-9$ & Bromomethane & 0.50 & $\mathrm{U}$ \\
\hline $75-00-3$ & Chloroethane & 0.50 & $\mathrm{U}$ \\
\hline $75-69-4$ & Trichlorofluoromethane & 0.50 & $\mathrm{U}$ \\
\hline $75-35-4$ & 1,1-Dichloroethene & 0.50 & $\mathrm{U}$ \\
\hline $76-13-1$ & $1,1,2$-Trichloro-1,2,2-trifluoroethane & 0.50 & $\mathrm{U}$ \\
\hline $67-64-1$ & Acetone & 5.0 & $\mathrm{U}$ \\
\hline $75-15-0$ & Carbon disulfide & 0.50 & $\mathrm{U}$ \\
\hline $79-20-9$ & Methyl acetate & 0.50 & $\mathrm{U}$ \\
\hline $75-09-2$ & Methylene Chloride & 0.50 & $\mathrm{U}$ \\
\hline $156-60-5$ & trans-1,2-Dichloroethene & 0.50 & $\mathrm{U}$ \\
\hline $1634-04-4$ & Methyl tert-butyl ether & 0.50 & $\mathrm{U}$ \\
\hline $75-34-3$ & 1,1-Dichloroethane & 0.50 & $\mathrm{U}$ \\
\hline $156-59-2$ & cis-1,2-Dichloroethene & 0.50 & $\mathrm{U}$ \\
\hline $78-93-3$ & 2-Butanone & 5.0 & $\mathrm{U}$ \\
\hline $74-97-5$ & Bromochloromethane & 0.50 & $\mathrm{U}$ \\
\hline $67-66-3$ & Chloroform & 0.50 & $\mathrm{U}$ \\
\hline $71-55-6$ & $1,1,1$-Trichloroethane & 0.50 & $\mathrm{U}$ \\
\hline $110-82-7$ & Cyclohexane & 0.50 & $\mathrm{U}$ \\
\hline $56-23-5$ & Carbon tetrachloride & 0.33 & J \\
\hline $71-43-2$ & Benzene & 0.50 & $\mathrm{U}$ \\
\hline $107-06-2$ & 1,2 -Dichloroethane & 0.50 & $\mathrm{U}$ \\
\hline
\end{tabular}

Report 1,4-Dioxane for Low-Medium VOA analysis only 
Lab Name: TESTAMERICA BURLINGTON

Lab Code: STLV Case No

Matrix: (SOIL/SED/WATER)

Sample wt/vol: 25.0

Water

$(\mathrm{g} / \mathrm{mL}) \quad \mathrm{mL}$

Level: (TRACE/LOW/MED) TRACE

\& Moisture: not dec.

GC Column: DB-624 ID: $0.20 \quad(\mathrm{~mm})$

Soil Extract Volume: (uL) (mL)

Purge Volume: 25.0
Contract: 8E-00302

SDG No.: 200-7268

\begin{tabular}{|c|c|c|c|}
\hline CAS NO. & COMPOUND & $\begin{array}{l}\text { CONCENTRATION UNITS: } \\
(\mathrm{ug} / \mathrm{L} \text { or } \mathrm{ug} / \mathrm{kg}) \mathrm{ug} / \mathrm{L}\end{array}$ & $Q$ \\
\hline $79-01-6$ & Trichloroethene & 0.50 & $\mathrm{U}$ \\
\hline $108-87-2$ & Methylcyclohexane & 0.50 & $\mathrm{U}$ \\
\hline $78-87-5$ & 1,2-Dichloropropane & 0.50 & $\mathrm{U}$ \\
\hline $75-27-4$ & Bromodichloromethane & 0.50 & $\mathrm{U}$ \\
\hline $10061-01-5$ & cis-1,3-Dichloropropene & 0.50 & $\mathrm{U}$ \\
\hline $108-10-1$ & 4-Methyl-2-pentanone & 5.0 & $\mathrm{U}$ \\
\hline $108-88-3$ & Toluene & 0.0070 & $\mathrm{~J} \mathrm{~B}$ \\
\hline $10061-02-6$ & trans-1,3-Dichloropropene & 0.50 & $\mathrm{U}$ \\
\hline $79-00-5$ & 1,1,2-Trichloroethane & 0.50 & $\mathrm{U}$ \\
\hline $127-18-4$ & Tetrachloroethene & 0.50 & $\mathrm{U}$ \\
\hline $591-78-6$ & 2-Hexanone & 5.0 & $\mathrm{U}$ \\
\hline $124-48-1$ & Dibromochloromethane & 0.50 & $\mathrm{U}$ \\
\hline $106-93-4$ & 1,2-Dibromoethane & 0.50 & $\mathrm{U}$ \\
\hline $108-90-7$ & Chlorobenzene & 0.50 & $\mathrm{U}$ \\
\hline $100-41-4$ & Ethylbenzene & 0.50 & $\mathrm{U}$ \\
\hline $95-47-6$ & o-Xylene & 0.50 & $\mathrm{U}$ \\
\hline $179601-23-1$ & m,p-Xylene & 0.50 & $\mathrm{U}$ \\
\hline $100-42-5$ & Styrene & 0.50 & $\mathrm{U}$ \\
\hline $75-25-2$ & Bromoform & 0.50 & $\mathrm{U}$ \\
\hline $98-82-8$ & Isopropylbenzene & 0.50 & $\mathrm{U}$ \\
\hline $79-34-5$ & $1,1,2,2$-Tetrachloroethane & 0.50 & $\mathrm{U}$ \\
\hline $541-73-1$ & 1,3-Dichlorobenzene & 0.50 & $\mathrm{U}$ \\
\hline $106-46-7$ & 1,4-Dichlorobenzene & 0.50 & U \\
\hline $95-50-1$ & 1,2-Dichlorobenzene & 0.50 & $\mathrm{U}$ \\
\hline $96-12-8$ & 1,2-Dibromo-3-Chloropropane & 0.50 & $\mathrm{U}$ \\
\hline $120-82-1$ & 1,2,4-Trichlorobenzene & 0.50 & U \\
\hline $87-61-6$ & 1,2,3-Trichlorobenzene & 0.50 & $\mathrm{U}$ \\
\hline
\end{tabular}


$1 \mathrm{~J}$ - FORM I VOA-TIC

VOLATILE ORGANICS ANALYSIS DATA SHEET

TENTATIVELY IDENTIFIED COMPOUNDS
EPA SAMPLE NO. BAMW17-W-28817

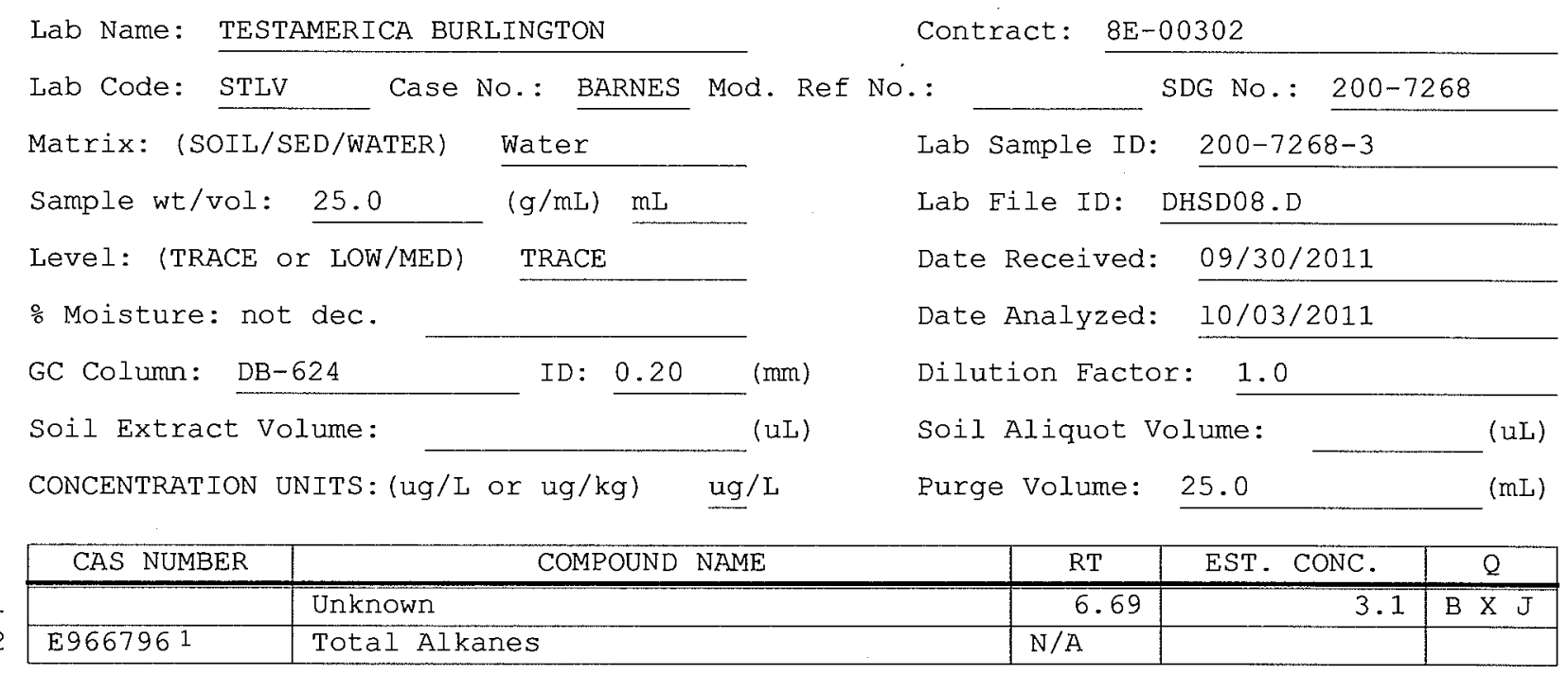

lEPA-designated Registry Number. 
$1 \mathrm{~A}$ - EORM I VOA-1

VOLATILE ORGANICS ANALYSIS DATA SHEET
EPA SAMPLE NO.

BAQCTB-W-28825
Lab Name: TESTAMERICA BURLINGTON

Lab Code: STLV Case No.: BARNES Mod. Ref No.:
Matrix: (SOIL/SED/WATER)

Sample wt/vol: 25.0

Water

$(\mathrm{g} / \mathrm{mL}) \mathrm{mL}$

Level: (TRACE/LOW/MED) TRACE

음 Moisture: not dec.

GC Column: DB-624 ID $: 0.20 \quad(\mathrm{~mm})$

Soil Extract Volume:

Purge Volume: 25.0 (mL)
Contract: $8 E-00302$

SDG No.: 200-7268

Lab Sample ID: 200-7268-4

Lab File ID: DHSD05.D

Date Received: 09/30/2011

Date Analyzed: 10/03/2011

Dilution Factor: 1.0

Soil Aliquot Volume: (uL)

\begin{tabular}{|c|c|c|c|}
\hline CAS NO. & COMPOUND & $\begin{array}{l}\text { CONCENTRATION UNITS: } \\
(\mathrm{ug} / \mathrm{L} \text { or } \mathrm{ug} / \mathrm{kg}) \mathrm{ug} / \mathrm{L}\end{array}$ & $\varnothing$ \\
\hline $75-71-8$ & Dichlorodifluoromethane & 0.50 & $\bar{U}$ \\
\hline $74-87-3$ & Chloromethane & 0.50 & $\mathrm{U}$ \\
\hline $75-01-4$ & Vinyl chloride & 0.50 & $\mathrm{U}$ \\
\hline $74-83-9$ & Bromomethane & 0.50 & $\mathrm{U}$ \\
\hline $75-00-3$ & Chloroethane & 0.50 & $\mathrm{U}$ \\
\hline $75-69-4$ & Trichlorofluoromethane & 0.50 & $\mathrm{U}$ \\
\hline $75-35-4$ & 1,1-Dichloroethene & 0.50 & $\mathrm{U}$ \\
\hline $76-13-1$ & $1,1,2$-Trichloro-1,2,2-trifluoroethane & 0.50 & $\mathrm{U}$ \\
\hline $67-64-1$ & Acetone & 4.6 & $\mathrm{JB}$ \\
\hline $75-15-0$ & Carbon disulfide & 0.056 & $\mathrm{~J} \mathrm{~B}$ \\
\hline $79-20-9$ & Methyl acetate & 0.50 & $\mathrm{U}$ \\
\hline $75-09-2$ & Methylene Chloride & 0.50 & $\mathrm{U}$ \\
\hline $156-60-5$ & trans-1,2-Dichloroethene & 0.50 & $\mathrm{U}$ \\
\hline $1634-04-4$ & Methyl tert-butyl ether & 0.50 & $\mathrm{U}$ \\
\hline $75-34-3$ & 1,1-Dichloroethane & 0.50 & $\mathrm{U}$ \\
\hline $156-59-2$ & cis-1,2-Dichloroethene & 0.50 & $\mathrm{U}$ \\
\hline $78-93-3$ & 2-Butanone & 5.0 & $\mathrm{U}$ \\
\hline $74-97-5$ & Bromochloromethane & 0.50 & $\mathrm{U}$ \\
\hline $67-66-3$ & Chloroform & 0.50 & $\mathrm{U}$ \\
\hline $71-55-6$ & $1,1,1$-Trichloroethane & 0.50 & $\mathrm{U}$ \\
\hline $110-82-7$ & Cyclohexane & 0.50 & $\mathrm{U}$ \\
\hline $56-23-5$ & Carbon tetrachloride & 0.50 & $\mathrm{U}$ \\
\hline $71-43-2$ & Benzene & 0.078 & $\mathrm{~J}$ \\
\hline $107-06-2$ & 1,2-Dichloroethane & 0.50 & $\mathrm{U}$ \\
\hline
\end{tabular}

Report 1,4-Dioxane for Low-Medium VOA analysis only 
Lab Name: TESTAMERICA BURLINGTON

Lab Code: STLV

Case No.: BARNES

Mod. Ref No.:
Contract: $\quad 8 \mathrm{E}-00302$

SDG No.: $200-7268$
Matrix: (SOIL/SED/WATER)

Sample wt/vol:

25.0

water

$(\mathrm{g} / \mathrm{mL}) \quad \mathrm{mL}$

Level: (TRACE/LOW/MED) TRACE

\% Moisture: not dec.

GC Column: DB-624 ID $: 0.20$ (mm)

Soil Extract Volume: (uL) (mL)

Purge Volume: 25.0
Lab Sample ID: 200-7268-4

Lab File ID: DHSD05.D

Date Received: 09/30/2011

Date Analyzed: 10/03/2011

Dilution Factor: 1.0

Soil Aliquot Volume:

(uL)

\begin{tabular}{|c|c|c|c|}
\hline CAS NO. & COMPOUND & $\begin{array}{l}\text { CONCENTRATION UNITS: } \\
(\mathrm{ug} / \mathrm{L} \text { or } \mathrm{ug} / \mathrm{kg}) \mathrm{ug} / \mathrm{L}\end{array}$ & $Q$ \\
\hline $79-01-6$ & Trichloroethene & 0.50 & $\overline{\mathrm{UU}}$ \\
\hline $108-87-2$ & Methylcyclohexane & 0.50 & $\mathrm{U}$ \\
\hline $78-87-5$ & 1,2-Dichloropropane & 0.50 & $\mathrm{U}$ \\
\hline $75-27-4$ & Bromodichloromethane & 0.50 & $\mathrm{U}$ \\
\hline $10061-01-5$ & cis-1,3-Dichloropropene & 0.50 & $\mathrm{U}$ \\
\hline $108-10-1$ & 4-Methyl-2-pentanone & 5.0 & $\mathrm{U}$ \\
\hline $108-88-3$ & Toluene & 0.53 & $\mathrm{~B}$ \\
\hline $10061-02-6$ & trans-1,3-Dichloropropene & 0.50 & $\mathrm{U}$ \\
\hline $79-00-5$ & $1,1,2$-Trichloroethane & 0.50 & $\mathrm{U}$ \\
\hline $127-18-4$ & Tetrachloroethene & 0.50 & $\mathrm{U}$ \\
\hline $591-78-6$ & 2-Hexanone & $5 \cdot .0$ & $\mathrm{U}$ \\
\hline $124-48-1$ & Dibromochloromethane & 0.50 & $\mathrm{U}$ \\
\hline $106-93-4$ & 1,2-Dibromoethane & 0.50 & $\mathrm{U}$ \\
\hline $108-90-7$ & Chlorobenzene & 0.50 & $\mathrm{U}$ \\
\hline $100-41-4$ & Ethylbenzene & 0.030 & J \\
\hline $95-47-6$ & o-Xylene & 0.024 & $\mathrm{~J}$ \\
\hline $179601-23-1$ & $\mathrm{~m}, \mathrm{p}$-Xylene & 0.085 & $\mathrm{~J}$ \\
\hline $100-42-5$ & Styrene & 0.18 & $\mathrm{~J}$ \\
\hline $75-25-2$ & Bromo form & 0.50 & $\mathrm{U}$ \\
\hline $98-82-8$ & Isopropylbenzene & 0.50 & $\mathrm{U}$ \\
\hline $79-34-5$ & $1,1,2,2$-Tetrachloroethane & 0.50 & $\mathrm{U}$ \\
\hline $541-73-1$ & 1,3-Dichlorobenzene & 0.50 & $\mathrm{U}$ \\
\hline $106-46-7$ & 1,4-Dichlorobenzene & 0.50 & $\mathrm{U}$ \\
\hline $95-50-1$ & 1,2-Dichlorobenzene & 0.50 & $\mathrm{U}$ \\
\hline $96-12-8$ & 1,2-Dibromo-3-Chloropropane & 0.50 & $\mathrm{U}$ \\
\hline $120-82-1$ & $1,2,4$-Trichlorobenzene & 0.50 & $\mathrm{U}$ \\
\hline $87-61-6$ & 1,2,3-Trichlorobenzene & 0.50 & $\mathrm{U}$ \\
\hline
\end{tabular}


IJ - FORM I VOA-TIC

VOLATILE ORGANICS ANALYSIS DATA SHEET

TENTATIVELY IDENTIFIED COMPOUNDS
EPA SAMPLE NO. BAQCTB-W-28825

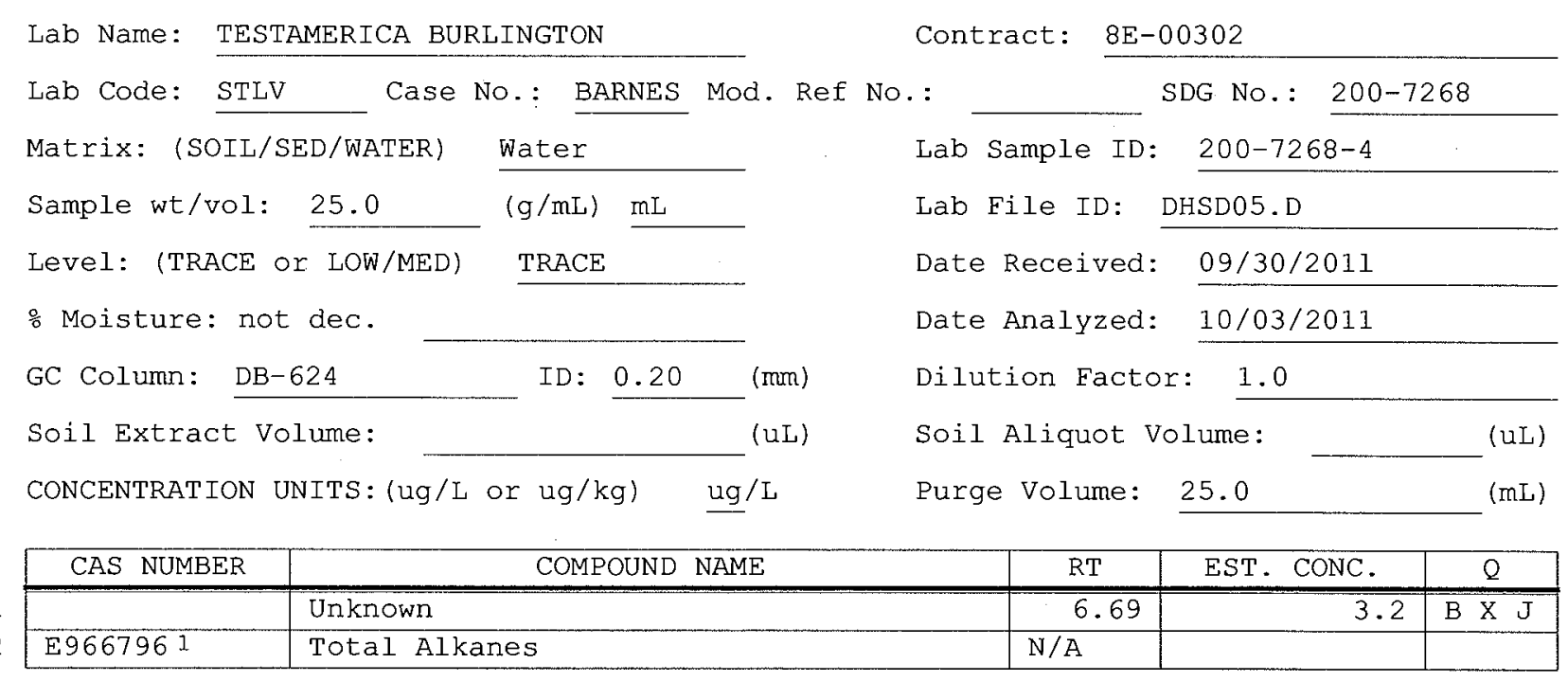

1 EPA-designated Registry Number. 
$6 A$ - FORM VI VOA-1

VOLATILE ORGANICS INITIAL CALIBRATION DATA

Lab Name: TESTAMERICA BURLINGTON

Contract: $8 \mathrm{E}-00302$

\begin{tabular}{|c|c|c|c|c|c|c|}
\hline Lab Code: & STLV & Case No.: BARNES & Mod. Ref No.: & & SDG No.: & $.00-7268$ \\
\hline Instrument & D. i & & Calibration & Date (s): & $09 / 22 / 2011$ & $09 / 22 / 2011$ \\
\hline Heated Pur & ge: $(Y / N)$ & $\underline{\mathrm{N}}$ & Calibration & Time (s): & 0848 & 1038 \\
\hline
\end{tabular}

Purge Volume: 25.0 $(\mathrm{mL})$

GC Column: $\mathrm{DB}-624$

ID: 0.20

(mm) Length: 25

(In)

\begin{tabular}{|c|c|c|c|c|c|c|c|}
\hline \multirow{2}{*}{$\begin{array}{l}\text { LAB FILE ID: } \\
\operatorname{RRE} 5.0=\text { DHS07.D }\end{array}$} & \multicolumn{3}{|c|}{$\mathrm{RRF} \underline{0.5}=\mathrm{DHSO5. \textrm {D }}$} & \multicolumn{4}{|c|}{ RRE $1.0=$ DHS06.D } \\
\hline & $\operatorname{RRF} 10=\mathrm{D}$ & HSO8.D & & RRE 20 & $=\mathrm{DHSO}$ & . $D$ & \\
\hline COMPOUND & RRF 0.5 & RRF 1.0 & RRE 5.0 & RRE 10 & RRE 20 & $\overline{\mathrm{RRF}}$ & 옹 \\
\hline Dichlorodifluoromethane & 0.507 & 0.475 & 0.480 & 0.489 & 0.487 & 0.488 & $\overline{2.5}$ \\
\hline Chloromethane & 0.801 & 0.648 & 0.594 & 0.593 & 0.584 & 0.644 & 14.2 \\
\hline Vinyl chloride & 0.452 & 0.427 & 0.424 & 0.442 & 0.437 & 0.436 & 2.6 \\
\hline Bromomethane & 0.206 & 0.200 & 0.187 & 0.193 & 0.193 & 0.196 & 3.7 \\
\hline Chloroethane & 0.267 & 0.214 & 0.205 & 0.221 & 0.215 & 0.224 & 11.0 \\
\hline Trichlorofluoromethane & 0.542 & 0.522 & 0.528 & 0.542 & 0.542 & 0.535 & 1.7 \\
\hline 1,1-Dichloroethene & 0.257 & 0.254 & 0.260 & 0.261 & 0.253 & 0.257 & 1.3 \\
\hline $\begin{array}{l}1,1,2 \text {-Trichloro- } \\
1,2,2 \text {-trifluoroethane }\end{array}$ & 0.304 & 0.307 & 0.305 & 0.308 & 0.299 & 0.305 & 1.2 \\
\hline Acetone & 0.029 & 0.026 & 0.022 & 0.023 & 0.021 & 0.024 & 13.5 \\
\hline Carbon disulfide & 0.758 & 0.658 & 0.663 & 0.696 & 0.711 & 0.697 & 5.8 \\
\hline Methyl acetate & 0.066 & 0.060 & 0.057 & 0.062 & 0.058 & 0.060 & 5.5 \\
\hline Methylene Chloride & 0.226 & 0.213 & 0.209 & 0.221 & 0.209 & 0.216 & 3.5 \\
\hline trans-1,2-Dichloroethene & 0.305 & 0.308 & 0.304 & 0.321 & 0.315 & 0.311 & 2.3 \\
\hline Methyl tert-butyl ether & 0.373 & 0.361 & 0.364 & 0.385 & 0.354 & 0.367 & 3.3 \\
\hline 1,1-Dichloroethane & 0.600 & 0.585 & 0.592 & 0.617 & 0.608 & 0.600 & 2.1 \\
\hline cis-1,2-Dichloroethene & 0.330 & 0.299 & 0.305 & 0.317 & 0.312 & 0.313 & 3.7 \\
\hline 2-Butanone & 0.040 & 0.040 & 0.039 & 0.042 & 0.039 & 0.040 & 3.7 \\
\hline Bromochloromethane & 0.113 & 0.103 & 0.103 & 0.107 & 0.102 & 0.106 & 4.1 \\
\hline Chloroform & 0.500 & 0.463 & 0.480 & 0.502 & 0.488 & 0.487 & 3.3 \\
\hline 1,1,1-Trichloroethane & 0.547 & 0.528 & 0.530 & 0.567 & 0.578 & 0.550 & 4.0 \\
\hline Cyclohexane & 0.846 & 0.839 & 0.869 & 0.918 & 0.917 & 0.878 & 4.3 \\
\hline Carbon tetrachloride & 0.509 & 0.475 & 0.509 & 0.535 & 0.547 & 0.515 & 5.4 \\
\hline Benzene & 1.568 & 1.515 & 1.482 & 1.544 & 1.514 & 1.525 & 2.2 \\
\hline 1,2-Dichloroethane & 0.272 & 0.252 & 0.271 & 0.279 & 0.268 & 0.268 & 3.7 \\
\hline Trichloroethene & 0.394 & 0.385 & 0.377 & 0.396 & 0.397 & 0.390 & 2.2 \\
\hline Methylcyclohexane & 0.721 & 0.692 & 0.715 & 0.753 & 0.755 & 0.727 & 3.7 \\
\hline
\end{tabular}

Report 1,4-Dioxane for Low-Medium VOA analysis only 
$6 \mathrm{~B}$ - FORM VI VOA-2

VOLATILE ORGANICS INITIAL CALIBRATION DATA

Lab Name: TESTAMERICA BURLINGTON

Contract: $8 \mathrm{E}-00302$

Lab Code: STLV Case No.: BARNES Mod. Ref No.

SDG No.: 200-7268

Instrument ID: D.i

Calibration Date(s):

$09 / 22 / 2011$

09/22/2011

Heated Purge: ( $Y / N$ ) N

Calibration Time(s):

0848

1038

Purge Volume: 25.0

(mL)

GC Column: DB-624

ID $: 0.20$

(mm) Length: 25

(m)

\begin{tabular}{|c|c|c|c|c|c|c|c|}
\hline LAB FILE ID: & $\operatorname{RRF} 0.5=\mathrm{L}$ & SO5.D & & RRE 1 . & $=\mathrm{DHSO}$ & & \\
\hline $\operatorname{RRF} 5.0=$ DHS07.D & $\operatorname{RRF} 10=\underline{I}$ & IS08.D & & RRF 20 & $=\underline{\mathrm{DHSO}}$ & & \\
\hline COMPOUND & $\mathrm{RRF} 0.5$ & $\mathrm{RRF} 1.0$ & $\mathrm{RRF} 5.0$ & RRF 10 & RRF 20 & $\overline{\mathrm{RRF}}$ & \%RSD \\
\hline 1,2-Dichloropropane & 0.366 & 0.346 & 0.353 & 0.373 & 0.363 & 0.360 & 3.0 \\
\hline Bromodichloromethane & 0.299 & 0.296 & 0.319 & 0.345 & 0.342 & 0.320 & 7.2 \\
\hline cis-1,3-Dichloropropene & 0.383 & 0.394 & 0.423 & 0.461 & 0.445 & 0.421 & 7.8 \\
\hline 4-Methyl-2-pentanone & 0.104 & 0.106 & 0.116 & 0.125 & 0.116 & 0.113 & 7.8 \\
\hline Toluene & 1.677 & 1.641 & 1.626 & 1.715 & 1.684 & 1.669 & 2.1 \\
\hline trans-1,3-Dichloropropene & 0.266 & 0.275 & 0.304 & 0.330 & 0.317 & 0.298 & 9.1 \\
\hline 1,1,2-Trichloroethane & 0.169 & 0.158 & 0.162 & 0.169 & 0.158 & 0.163 & 3.2 \\
\hline Tetrachloroethene & 0.322 & 0.307 & 0.308 & 0.321 & 0.319 & 0.315 & 2.3 \\
\hline 2-Hexanone & 0.072 & 0.074 & 0.081 & 0.086 & 0.078 & 0.078 & 6.9 \\
\hline Dibromochloromethane & 0.170 & 0.166 & 0.183 & 0.206 & 0.203 & 0.186 & 10.0 \\
\hline 1,2-Dibromoethane & 0.146 & 0.143 & 0.146 & 0.157 & 0.149 & 0.148 & 3.4 \\
\hline Chlorobenzene & 1.087 & 1.060 & 1.019 & 1.063 & 1.036 & 1.053 & 2.5 \\
\hline Ethylbenzene & 1.819 & 1.784 & 1.862 & 1.965 & 1.939 & 1.874 & 4.1 \\
\hline o-Xylene & 0.675 & 0.660 & 0.704 & 0.746 & 0.721 & 0.701 & 4.9 \\
\hline m,p-xylene & 0.741 & 0.722 & 0.754 & 0.796 & 0.782 & 0.759 & 3.9 \\
\hline Styrene & 0.934 & 0.954 & 1.052 & 1.115 & 1.078 & 1.027 & 7.7 \\
\hline Bromoform & 0.105 & 0.123 & 0.136 & 0.150 & 0.153 & 0.133 & 14.9 \\
\hline Isopropylbenzene & 1.782 & 1.820 & 1.941 & 2.045 & 2.033 & 1.924 & 6.3 \\
\hline $1,1,2,2$-Tetrachloroethane & 0.150 & 0.146 & 0.151 & 0.164 & 0.149 & 0.152 & 4.5 \\
\hline 1,3-Dichlorobenzene & 1.703 & 1.573 & 1.651 & 1.731 & 1.687 & 1.669 & 3.6 \\
\hline 1,4-Dichlorobenzene & 1.858 & 1.629 & 1.627 & 1.691 & 1.626 & 1.686 & 5.9 \\
\hline 1,2-Dich1orobenzene & 1.450 & 1.331 & 1.370 & 1.417 & 1.352 & 1.384 & 3.5 \\
\hline 1,2-Dibromo-3-Chloropropane & 0.043 & 0.036 & 0.038 & 0.043 & 0.042 & 0.040 & 8.2 \\
\hline 1,2,4-Trichlorobenzene & 0.793 & 0.688 & 0.756 & 0.806 & 0.785 & 0.766 & 6.1 \\
\hline 1,2,3-Trichlorobenzene & 0.568 & 0.536 & 0.576 & 0.603 & 0.584 & 0.573 & 4.3 \\
\hline
\end{tabular}


$6 \mathrm{C}-$ FORM VI VOA-3

VOLATILE ORGANICS INITIAL CALIBRATION DATA

Lab Name: TESTAMERICA BURLINGTON

Contract: $8 \mathrm{E}-00302$

Lab Code: STLV Case No.: BARNES Mod. Ref No.: SDG No.: 200-7268

Instrument ID: D.i

\begin{tabular}{|c|c|c|c|}
\hline Calibration & Date $(s)$ : & $09 / 22 / 2011$ & $09 / 22 / 2011$ \\
\hline ihr & (s) & 0848 & 1038 \\
\hline
\end{tabular}

Heated Purge: ( $Y / N) \quad N$

Purge Volume: 25.0 (mL)

GC Column: $\mathrm{DB}-624$

ID: 0.20

$(\mathrm{mm})$

Length: 25

(m)

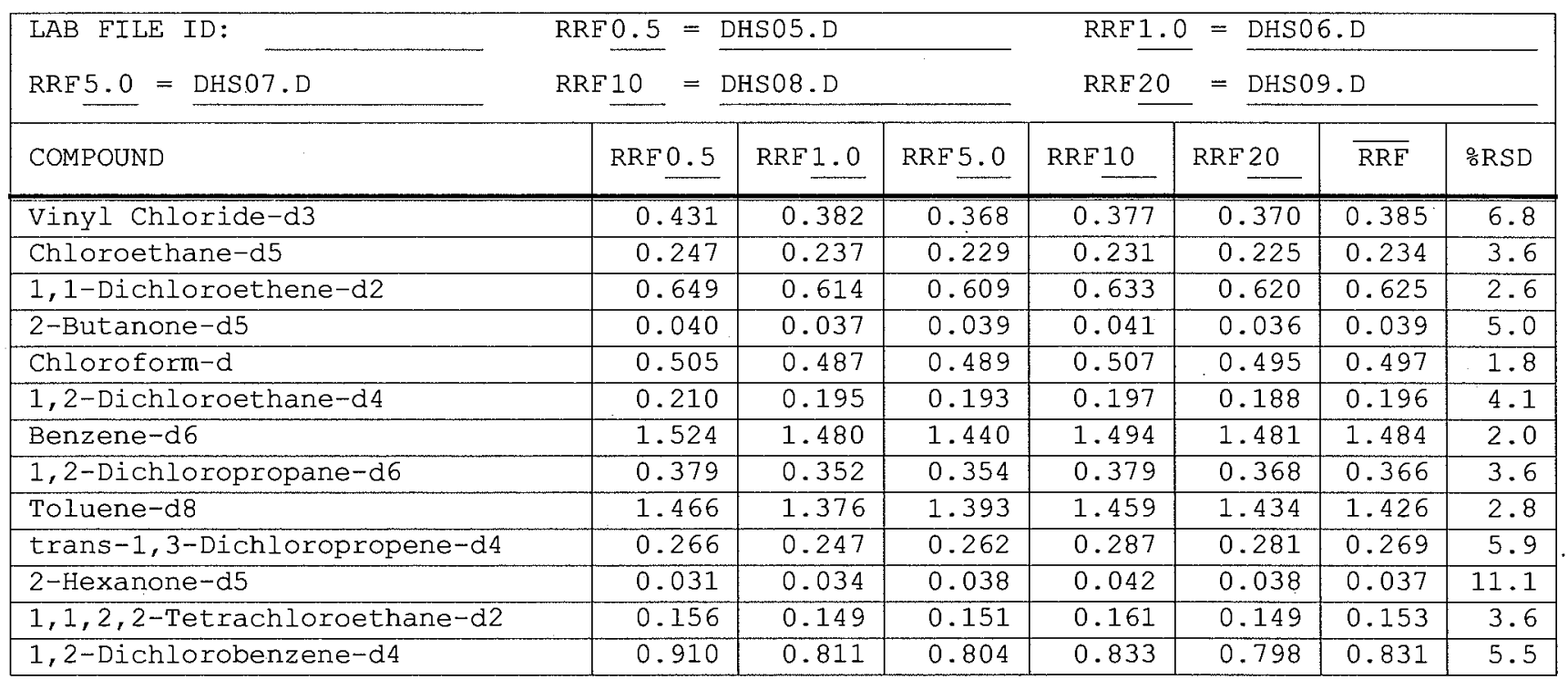

Report 1,4-Dioxane-d8 for Low-Medium VOA analysis only 
$7 A$ - EORM VII VOA-1

VOLATILE CONTINUING CALIBRATION DATA

Lab Name: TESTAMERICA BURLINGTON

Contract: $8 \mathrm{E}-00302$

Lab Code: STLV Case No.: BARNES Mod. Ref No.:

SDG No.: 200-7268

Instrument ID: D.i

Calibration Date: 10/03/2011 Time: 0854

Lab File Id: DHSD02.D

EPA Sample No. (VSTD\#\#\#\#): VSTD005DG Init. Calib. Date(s): 09/22/2011 09/22/2011

Heated Purge: ( $\mathrm{Y} / \mathrm{N}) \mathrm{N}$

GC Column: DB-624

Init. Calib. Time(s): $0848 \quad 1038$

Purge Volume: 25.0

(mL)

\begin{tabular}{|c|c|c|c|c|c|}
\hline COMPOUND & $\overline{\mathrm{RRE}}$ & RRF5. 0 & $\begin{array}{l}\text { MIN } \\
\text { RRF }\end{array}$ & $\because \mathrm{D}$ & MAX $\because D$ \\
\hline Dichlorodifluoromethane & 0.488 & 0.465 & 0.010 & -4.6 & 40.0 \\
\hline Chloromethane & 0.644 & 0.678 & 0.010 & 5.3 & 40.0 \\
\hline Vinyl chloride & 0.436 & 0.515 & 0.010 & 17.9 & 30.0 \\
\hline Bromomethane & 0.196 & 0.172 & 0.010 & $-12 \cdot 3$ & 30.0 \\
\hline Chloroethane & 0.224 & 0.264 & 0.010 & 17.5 & 40.0 \\
\hline Trichlorofluoromethane & 0.535 & 0.555 & 0.010 & 3.6 & 40.0 \\
\hline 1,1-Dichloroethene & 0.257 & 0.297 & 0.010 & 15.6 & 30.0 \\
\hline 1,1,2-Trich1oro-1,2,2-trifluoroethane & 0.305 & 0.338 & 0.010 & 10.7 & 40.0 \\
\hline Acetone & 0.024 & 0.023 & 0.010 & -3.1 & 40.0 \\
\hline Carbon disulfide & 0.697 & 0.798 & 0.010 & 14.5 & 40.0 \\
\hline Methyl acetate & 0.060 & 0.064 & 0.010 & 6.0 & 40.0 \\
\hline Methylene Chloride & 0.216 & 0.221 & 0.010 & 2.4 & 40.0 \\
\hline trans $-1,2-$ Dichloroethene & 0.311 & 0.295 & 0.010 & -5.0 & 40.0 \\
\hline Methyl tert-butyl ether & 0.367 & 0.282 & 0.010 & $-23,3$ & 40.0 \\
\hline 1,1-Dichloroethane & 0.600 & 0.582 & 0.010 & -3.1 & 30.0 \\
\hline cis-1,2-Dichloroethene & 0.313 & 0.285 & 0.010 & -9.0 & 40.0 \\
\hline 2-Butanone & 0.040 & 0.037 & 0.010 & -8.2 & 40.0 \\
\hline Bromochloromethane & 0.106 & 0.092 & 0.010 & -13.4 & 30.0 \\
\hline Chloroform & 0.487 & 0.444 & 0.010 & -8.8 & 30.0 \\
\hline $1,1,1$-Trichloroethane & 0.550 & 0.475 & 0.010 & -13.5 & 30.0 \\
\hline Cyclohexane & 0.878 & 0.875 & 0.010 & -0.4 & 40.0 \\
\hline Carbon tetrachloride & 0.515 & 0.447 & 0.010 & -13.1 & 30.0 \\
\hline Benzene & 1.525 & 1.415 & 0.010 & -7.2 & 30.0 \\
\hline 1,2-Dichloroethane & 0.268 & 0.241 & 0.010 & -10.0 & 30.0 \\
\hline Trichloroethene & 0.390 & 0.336 & 0.010 & -13.8 & 30.0 \\
\hline Methylcyclohexane & 0.727 & 0.719 & 0.010 & -1.1 & 40.0 \\
\hline
\end{tabular}

Report 1,4-Dioxane for Low/Medium VOA analysis only 
$7 \mathrm{~B}$ - FORM VII VOA-2

VOLATILE CONTINUING CALIBRATION DATA

Lab Name: TESTAMERICA BURLINGTON

Contract: $\quad 8 \mathrm{E}-00302$

Lab Code: STLV Case No.: BARNES Mod. Ref No.:

SDG No.: 200-7268

Instrument ID: D.i

Calibration Date: 10/03/2011 Time: 0854

Lab File Id: DHSD02.D Init. Calib. Date(s): 09/22/2011 09/22/2011

EPA Sample No. (VSTD\#\#\#): VSTD005DG

Init. Calib. Time(s): $0848 \quad 1038$

Heated Purge: ( $\mathrm{Y} / \mathrm{N})$

GC Column: DB-624

ID: $0.20(\mathrm{~mm})$ Length: 25

(m)

Purge Volume: 25.0

(mL)

\begin{tabular}{|c|c|c|c|c|c|}
\hline COMPOUND & $\overline{\mathrm{RRF}}$ & RRF5.0 & $\begin{array}{l}\text { MIN } \\
\text { RRE }\end{array}$ & $\frac{\circ}{b}$ & $\operatorname{MAX} \% \mathrm{D}$ \\
\hline 1,2-Dichloropropane & 0.360 & 0.347 & 0.010 & -3.7 & $\overline{40.0}$ \\
\hline Bromodichloromethane & 0.320 & 0.274 & 0.010 & -14.3 & 30.0 \\
\hline cis-1,3-Dichloropropene & 0.421 & 0.372 & 0.010 & -11.6 & 30.0 \\
\hline 4-Methyl-2-pentanone & 0.113 & 0.105 & 0.010 & -7.6 & 40.0 \\
\hline Toluene & 1.669 & 1.531 & 0.010 & -8.3 & 30.0 \\
\hline trans-1,3-Dichloropropene & 0.298 & 0.262 & 0.010 & $-12 \cdot 3$ & 30.0 \\
\hline 1,1,2-Trichloroethane & 0.163 & 0.140 & 0.010 & -14.6 & 30.0 \\
\hline Tetrachloroethene & 0.315 & 0.286 & 0.010 & -9.3 & 30.0 \\
\hline 2-Hexanone & 0.078 & 0.071 & 0.010 & -9.0 & 40.0 \\
\hline Dibromochloromethane & 0.186 & 0.157 & 0.010 & -15.7 & 30.0 \\
\hline 1,2-Dibromoethane & 0.148 & 0.120 & 0.010 & -18.8 & 40.0 \\
\hline Chlorobenzene & 1.053 & 0.950 & 0.010 & -9.7 & 30.0 \\
\hline Ethylbenzene & 1.874 & 1.755 & 0.010 & -6.4 & 30.0 \\
\hline o-Xylene & 0.701 & 0.681 & 0.010 & -2.9 & 30.0 \\
\hline $\mathrm{m}, \mathrm{p}$-Xylene & 0.759 & 0.722 & 0.010 & -4.8 & 30.0 \\
\hline Styrene & 1.027 & 0.966 & 0.010 & -5.9 & 30.0 \\
\hline Bromoform & 0.133 & 0.114 & 0.010 & -14.8 & 30.0 \\
\hline Isopropylbenzene & 1.924 & 1.892 & 0.010 & -1.7 & 40.0 \\
\hline $1,1,2,2$-Tetrachloroethane & 0.152 & 0.136 & 0.010 & -10.6 & 30.0 \\
\hline 1,3-Dichlorobenzene & 1.669 & 1.454 & 0.010 & -12.9 & 30.0 \\
\hline 1,4-Dichlorobenzene & 1.686 & 1.451 & 0.010 & -14.0 & 30.0 \\
\hline 1,2-Dichlorobenzene & 1.384 & 1.219 & 0.010 & -11.9 & 30.0 \\
\hline 1,2-Dibromo-3-Chloropropane & 0.040 & 0.029 & 0.010 & -27.7 & 40.0 \\
\hline $1,2,4$-Trichlorobenzene & 0.766 & 0.626 & 0.010 & -18.2 & 30.0 \\
\hline $1,2,3$-Trichlorobenzene & 0.573 & 0.460 & 0.010 & -19.7 & 30.0 \\
\hline
\end{tabular}


$7 C$ - FORM VII VOA-3

VOLATILE CONTINUING CALIBRATION DATA

Lab Name: TESTAMERICA BURIINGTON

Contract: $\quad 8 \mathrm{E}-00302$

Lab Code: STLV Case No.: BARNES Mod. Ref No.:

SDG No.: $200-7268$

Instrument ID: D.i

Calibration Date: 10/03/2011 Time: 0854

Lab File Id: DHSD02.D Init. Calib. Date(s): 09/22/2011 09/22/2011

EPA Sample No. (VSTD\#\#\#\#):

VSTD005DG

Init. Calib. Time(s): 0848 1038

Heated Purge: $(\mathrm{Y} / \mathrm{N}) \mathrm{N}$

GC Column: DB-624

ID: $0.20(\mathrm{~mm})$ Iength: 25

(m)

Purge Volume: 25.0

(mL)

\begin{tabular}{|c|c|c|c|c|c|}
\hline COMPOUND & $\overline{\mathrm{RRF}}$ & RRE5.0 & $\begin{array}{l}\text { MIN } \\
\text { RRF }\end{array}$ & $\frac{\circ}{\partial}$ & MAX $\div D$ \\
\hline Vinyl Chloride-d3 & 0.385 & 0.446 & 0.010 & 15.6 & 30.0 \\
\hline Chloroethane-d5 & 0.234 & 0.281 & 0.010 & 20.1 & 40.0 \\
\hline 1,1-Dichloroethene-d2 & 0.625 & 0.686 & 0.010 & 9.8 & 30.0 \\
\hline 2-Butanone-d5 & 0.039 & 0.035 & 0.010 & -9.2 & 40.0 \\
\hline Chloroform-d & 0.497 & 0.447 & 0.010 & -10.0 & 30.0 \\
\hline 1,2-Dichloroethane-d4 & 0.196 & 0.167 & 0.010 & -14.8 & 30.0 \\
\hline Benzene-d6 & 1.484 & 1.340 & 0.010 & -9.7 & 30.0 \\
\hline 1,2-Dichloropropane-d6 & 0.366 & 0.335 & 0.010 & -8.5 & 40.0 \\
\hline Toluene-d8 & 1.426 & 1.297 & 0.010 & -9.0 & 30.0 \\
\hline trans-1,3-Dichloropropene-d4 & 0.269 & 0.217 & 0.010 & -19.3 & 30.0 \\
\hline 2-Hexanone-d5 & 0.037 & 0.029 & 0.010 & -21.6 & 40.0 \\
\hline $1,1,2,2-$ Tetrachloroethane-d2 & 0.153 & 0.133 & 0.010 & -13.2 & 30.0 \\
\hline 1,2-Dichlorobenzene-d4 & 0.831 & 0.697 & 0.010 & -16.1 & 30.0 \\
\hline
\end{tabular}

Report 1,4-Dioxane-d8 for Low/Medium VOA analysis only 
$7 \mathrm{~A}$ - FORM VII VOA-1

VOLATILE CONTINUING CALIBRATION DATA

Lab Name: TESTAMERICA BURLINGTON

Contract: $8 \mathrm{E}-00302$

Lab Code: STLV Case No.: BARNES Mod. Ref No.:

SDG No.: 200-7268

Instrument ID: D.i

Calibration Date: 10/03/2011 Time: 1521

Lab File Id: DHSD16.D Init. Calib. Date(s): 09/22/2011 09/22/2011

EPA Sample No. (VSTD\#\#\#\#) :

VSTD005GD

Init. Calib. Time(s): $0848 \quad 1038$

Heated Purge: $(\mathrm{Y} / \mathrm{N}) \mathrm{N}$

GC Column: DB-624

ID: $0.20(\mathrm{~mm})$ Length: 25 (m)

Purge Volume: 25.0

$(\mathrm{mL})$

\begin{tabular}{|c|c|c|c|c|c|}
\hline COMPOUND & $\mathrm{RRF}$ & RRF5.0 & $\begin{array}{l}\text { MIN } \\
\text { RRF }\end{array}$ & $\because \mathrm{D}$ & $\operatorname{MAX} \div \mathrm{D}$ \\
\hline Dichlorodifluoromethane & 0.488 & 0.477 & 0.010 & -2.2 & 50.0 \\
\hline Chloromethane & 0.644 & 0.717 & 0.010 & 11.3 & 50.0 \\
\hline Vinyl chloride & 0.436 & 0.516 & 0.100 & 18.2 & 50.0 \\
\hline Bromomethane & 0.196 & 0.189 & 0.100 & -3.2 & 50.0 \\
\hline Chloroethane & 0.224 & 0.275 & 0.010 & 22.5 & 50.0 \\
\hline Trichlorofluoromethane & 0.535 & 0.584 & 0.010 & 9.1 & 50.0 \\
\hline 1,1-Dichloroethene & 0.257 & 0.298 & 0.100 & 16.0 & 50.0 \\
\hline 1,1,2-Trichloro-1,2,2-trifluoroethane & 0.305 & 0.350 & 0.010 & 14.8 & 50.0 \\
\hline Acetone & 0.024 & 0.030 & 0.010 & 25.7 & 50.0 \\
\hline Carbon disulfide & 0.697 & 0.776 & 0.010 & 11.3 & 50.0 \\
\hline Methyl acetate & 0.060 & 0.082 & 0.010 & 36.3 & 50.0 \\
\hline Methylene Chloride & 0.216 & 0.242 & 0.010 & 12.4 & 50.0 \\
\hline trans-1,2-Dichloroethene & 0.311 & 0.309 & 0.010 & -0.6 & 50.0 \\
\hline Methyl tert-butyl ether & 0.367 & 0.346 & 0.010 & -5.7 & 50.0 \\
\hline 1,1-Dichloroethane & 0.600 & 0.611 & 0.200 & 1.8 & 50.0 \\
\hline cis-1,2-Dichloroethene & 0.313 & 0.299 & 0.010 & -4.5 & 50.0 \\
\hline 2-Butanone & 0.040 & 0.048 & 0.010 & 20.2 & 50.0 \\
\hline Bromochloromethane & 0.106 & 0.100 & 0.050 & -5.4 & 50.0 \\
\hline Chloroform & 0.487 & 0.473 & 0.200 & -2.7 & 50.0 \\
\hline $1,1,1$-Trichloroethane & 0.550 & 0.455 & 0.100 & -17.3 & 50.0 \\
\hline Cyclohexane & 0.878 & 0.834 & 0.010 & -5.0 & 50.0 \\
\hline Carbon tetrachloride & 0.515 & 0.437 & 0.100 & -15.1 & 50.0 \\
\hline Benzene & 1.525 & 1.377 & 0.400 & -9.7 & 50.0 \\
\hline 1,2-Dichloroethane & 0.268 & 0.285 & 0.100 & 6.4 & 50.0 \\
\hline Trichloroethene & 0.390 & 0.319 & 0.300 & -18.1 & 50.0 \\
\hline Methylcyclohexane & 0.727 & 0.704 & 0.010 & -3.2 & 50.0 \\
\hline
\end{tabular}

Report 1,4-Dioxane for Low/Medium VOA analysis only 
$7 B$ - FORM VII VOA-2

VOLATILE CONTINUING CALIBRATION DATA

Lab Name: TESTAMERICA BURLINGTON

Contract: $8 \mathrm{E}-00302$

Lab Code: STLV Case No.: BARNES Mod. Ref No.:

SDG No.: 200-7268

Instrument ID: D.i

Calibration Date: 10/03/2011 Time: 1521

Lab File Id: DHSD16.D

EPA Sample No. (VSTD\#\#\#\#): VSTD005GD

Init. Calib. Date(s): 09/22/2011 09/22/2011

Heated Purge: $(\mathrm{Y} / \mathrm{N}) \quad \mathrm{N}$

GC Column: DB-624

Init. Calib. Time(s): 0848 1038

Purge Volume: 25.0

(mL)

\begin{tabular}{|c|c|c|c|c|c|}
\hline COMPOUND & $\overline{\mathrm{RRF}}$ & RRE5.0 & $\begin{array}{l}\text { MIN } \\
\text { RRE }\end{array}$ & $\% \mathrm{D}$ & MAX $\because D$ \\
\hline 1,2-Dichloropropane & 0.360 & 0.356 & 0.010 & -1.2 & 50.0 \\
\hline Bromodichloromethane & 0.320 & 0.294 & 0.200 & -8.1 & 50.0 \\
\hline cis-1,3-Dichloropropene & 0.421 & 0.388 & 0.200 & -7.9 & 50.0 \\
\hline 4-Methyl-2-pentanone & 0.113 & 0.128 & 0.010 & 12.9 & 50.0 \\
\hline Toluene & 1.669 & 1.540 & 0.400 & -7.7 & 50.0 \\
\hline trans-1,3-Dichloropropene & 0.298 & 0.280 & 0.100 & -6.1 & 50.0 \\
\hline 1,1,2-Trichloroethane & 0.163 & 0.151 & 0.100 & -7.7 & 50.0 \\
\hline Tetrachloroethene & 0.315 & 0.285 & 0.100 & -9.8 & 50.0 \\
\hline 2-Hexanone & 0.078 & 0.089 & 0.010 & 13.8 & 50.0 \\
\hline Dibromochloromethane & 0.186 & 0.183 & 0.100 & -1.2 & 50.0 \\
\hline 1,2-Dibromoethane & 0.148 & 0.134 & 0.010 & -9.8 & 50.0 \\
\hline Chlorobenzene & 1.053 & 0.984 & 0.500 & -6.6 & 50.0 \\
\hline Ethylbenzene & 1.874 & 1.759 & 0.100 & -6.1 & 50.0 \\
\hline o-xylene & 0.701 & 0.690 & 0.300 & -1.6 & 50.0 \\
\hline $\mathrm{m}, \mathrm{p}$-Xylene & 0.759 & 0.743 & 0.300 & -2.1 & 50.0 \\
\hline Styrene & 1.027 & 1.042 & 0.300 & 1.5 & 50.0 \\
\hline Bromoform & 0.133 & 0.130 & 0.050 & -2.7 & 50.0 \\
\hline Isopropylbenzene & 1.924 & 1.855 & 0.010 & -3.6 & 50.0 \\
\hline $1,1,2,2$-Tetrachloroethane & 0.152 & 0.158 & 0.100 & 4.2 & 50.0 \\
\hline 1,3-Dichlorobenzene & 1.669 & 1.417 & 0.400 & -15.1 & 50.0 \\
\hline 1,4-Dichlorobenzene & 1.686 & 1.516 & 0.400 & -10.1 & 50.0 \\
\hline 1,2-Dichlorobenzene & 1.384 & 1.290 & 0.400 & -6.8 & 50.0 \\
\hline 1,2-Dibromo-3-Chloropropane & 0.040 & 0.034 & 0.010 & -16.4 & 50.0 \\
\hline 1,2,4-Trichlorobenzene & 0.766 & 0.609 & 0.200 & -20.4 & 50.0 \\
\hline 1,2,3-Trichlorobenzene & 0.573 & 0.485 & 0.200 & -15.5 & 50.0 \\
\hline
\end{tabular}


$7 \mathrm{C}-$ FORM VII VOA-3

VOLATILE CONTINUING CALIBRATION DATA

Lab Name: TESTAMERICA BURLINGTON

Contract: $8 \mathrm{E}-00302$

Lab Code: STLV Case No.: BARNES Mod. Ref No.: SDG No.: 200-7268

Instrument ID: D.i

Calibration Date: 10/03/2011 Time: 1521

Lab File Id: DHSD16.D Init. Calib. Date(s): 09/22/2011 09/22/2011

EPA Sample No. (VSTD\#\#\#\#): VSTD005GD

Init. Calib. Time(s): $0848 \quad 1038$

Heated Purge: $(\mathrm{Y} / \mathrm{N})$

$\mathrm{N} \quad \mathrm{GC}$ Column: $\mathrm{DB}-624$

ID: $0.20(\mathrm{~mm})$ Length: 25 (m)

Purge Volume: 25.0

$(\mathrm{mL})$

\begin{tabular}{|c|c|c|c|c|c|}
\hline COMPOUND & $\overline{\mathrm{RRF}}$ & RRF5. 0 & $\begin{array}{l}\text { MIN } \\
\text { RRF }\end{array}$ & $\% \mathrm{D}$ & MAX $\because D$ \\
\hline Vinyl Chloride-d3 & 0.385 & 0.429 & 0.010 & 11.2 & 50.0 \\
\hline Chloroethane-d5 & 0.234 & 0.279 & 0.010 & 19.4 & 50.0 \\
\hline 1,1-Dichloroethene-d2 & 0.625 & 0.719 & 0.010 & 15.0 & 50.0 \\
\hline 2-Butanone-d5 & 0.039 & 0.043 & 0.010 & 12.1 & 50.0 \\
\hline Chloroform-d & 0.497 & 0.481 & 0.010 & -3.1 & 50.0 \\
\hline 1,2-Dichloroethane-d4 & 0.196 & 0.194 & 0.010 & -1.5 & 50.0 \\
\hline Benzene-d6 & 1.484 & 1.315 & 0.010 & -11.4 & 50.0 \\
\hline 1,2-Dichloropropane-d6 & 0.366 & 0.342 & 0.010 & -6.7 & 50.0 \\
\hline Toluene-d8 & 1.426 & 1.292 & 0.010 & -9.4 & 50.0 \\
\hline trans $-1,3-\mathrm{Di}$ chloropropene-d 4 & 0.269 & 0.232 & 0.010 & -13.5 & 50.0 \\
\hline 2-Hexanone-d5 & 0.037 & 0.035 & 0.010 & -4.3 & 50.0 \\
\hline $1,1,2,2-$ Tetrachloroethane-d2 & 0.153 & 0.160 & 0.010 & 4.2 & 50.0 \\
\hline 1.2-Dichlorobenzene-d4 & 0.831 & 0.747 & 0.010 & -10.2 & 50.0 \\
\hline
\end{tabular}

Report 1,4-Dioxane-d8 for Low/Medium VOA analysis only 
$1 A$ - FORM I VOA-1

VOLATILE ORGANICS ANALYSIS DATA SHEET
EPA SAMPLE NO.

VBLKDG

Lab Name: TESTAMERICA BURLINGTON

Contract: $8 \mathrm{E}-00302$

Lab Code: STLV Case No.: BARNES Mod. Ref No.: SDG No.: 200-7268

Matrix: (SOIL/SED/WATER) Water

Sample wt/vol: $25.0(\mathrm{~g} / \mathrm{mL}) \mathrm{mL}$

Level: (TRACE/LOW/MED) TRACE

o Moisture: not dec.

GC Column: DB-624

ID: $0.20 \quad(\mathrm{~mm})$

(uL)

(uL)

Soil Extract Volume: (mL)

Lab Sample ID: MB 200-26126/3

Purge Volume: 25.0

Lab File ID: DHSD03.D

Date Received:

Date Analyzed: 10/03/2011

Dilution Factor: 1.0

Soil Aliquot Volume:

\begin{tabular}{|c|c|c|c|}
\hline CAS NO. & COMPOUND & $\begin{array}{l}\text { CONCENTRATION UNITS: } \\
(\mathrm{ug} / \mathrm{L} \text { or } \mathrm{ug} / \mathrm{kg}) \mathrm{ug} / \mathrm{L}\end{array}$ & Q \\
\hline $75-71-8$ & Dichlorodifluoromethane & 0.50 & $\mathrm{U}$ \\
\hline $74-87-3$ & Chloromethane & 0.50 & $\mathrm{U}$ \\
\hline $75-01-4$ & Vinyl chloride & 0.50 & $\mathrm{U}$ \\
\hline $74-83-9$ & Bromomethane & 0.50 & $\mathrm{U}$ \\
\hline $75-00-3$ & Chloroethane & 0.50 & $\mathrm{U}$ \\
\hline $75-69-4$ & Trichlorofluoromethane & 0.50 & $\mathrm{U}$ \\
\hline $75-35-4$ & 1,1-Dichloroethene & 0.50 & $\mathrm{U}$ \\
\hline $76-13-1$ & 1,1,2-Trichloro-1,2,2-trifluoroethane & 0.50 & $\mathrm{U}$ \\
\hline $67-64-1$ & Acetone & 2.8 & $\mathrm{~J}$ \\
\hline $75-15-0$ & Carbon disulfide & 0.10 & $\mathrm{~J}$ \\
\hline $79-20-9$ & Methyl acetate & 0.50 & $\mathrm{U}$ \\
\hline $75-09-2$ & Methylene Chloride & 0.50 & $\mathrm{U}$ \\
\hline $156-60-5$ & trans-1,2-Dichloroethene & 0.50 & $\mathrm{U}$ \\
\hline $1634-04-4$ & Methyl tert-butyl ether & 0.50 & U \\
\hline $75-34-3$ & 1,1-Dichloroethane & 0.50 & $\mathrm{U}$ \\
\hline $156-59-2$ & cis-1,2-Dichloroethene & 0.50 & $\mathrm{U}$ \\
\hline $78-93-3$ & 2-Butanone & 5.0 & $\mathrm{U}$ \\
\hline $74-97-5$ & Bromochloromethane & 0.50 & $\mathrm{U}$ \\
\hline $67-66-3$ & Chloroform & 0.50 & U \\
\hline $71-55-6$ & $1,1,1-$ Trichloroethane & 0.50 & $\mathrm{U}$ \\
\hline $110-82-7$ & Cyclohexane & 0.50 & $\mathrm{U}$ \\
\hline $56-23-5$ & Carbon tetrachloride & 0.50 & $\mathrm{U}$ \\
\hline $71-43-2$ & Benzene & 0.50 & $\mathrm{U}$ \\
\hline $107-06-2$ & 1,2-Dichloroethane & 0.50 & $\mathrm{U}$ \\
\hline
\end{tabular}

Report 1,4-Dioxane for Low-Medium VOA analysis only 
$1 B$ - FORM I VOA-2

VOLATILE ORGANICS ANALYSIS DATA SHEET
EPA SAMPLE NO. VBLKDG

Lab Name: TESTAMERICA BURLINGTON

Lab Code: STLV Case

No.: BARNES Mod. Ref No.:

Contract: $8 \mathrm{E}-00302$ ID $: 0.20 \quad(\mathrm{~mm})$

Matrix: (SOIL/SED/WATER)

Sample wt/vol: 25.0

\section{Level: (TRACE/LOW/MED) TRACE}

o Moisture: not dec.

GC Column: DB-624

Soil Extract Volume:

Water

$(\mathrm{g} / \mathrm{mL}) \quad \mathrm{mL}$

Purge Volume: 25.0
(uL)

(mL)

SDG No.: 200-7268

Lab Sample ID: MB 200-26126/3

Lab File ID: DHSD03.D

Date Received:

Date Analyzed: 10/03/2011

Dilution Factor: 1.0

Soil Aliquot Volume: (UI)

\begin{tabular}{|c|c|c|c|}
\hline CAS NO. & COMPOUND & $\begin{array}{l}\text { CONCENTRATION UNITS: } \\
(\mathrm{ug} / \mathrm{L} \text { or } \mathrm{ug} / \mathrm{kg}) \mathrm{ug} / \mathrm{L}\end{array}$ & $Q$ \\
\hline $79-01-6$ & Trichloroethene & 0.50 & $\bar{U}$ \\
\hline $108-87-2$ & Methylcyclohexane & 0.50 & $\mathrm{U}$ \\
\hline $78-87-5$ & 1,2-Dichloropropane & 0.50 & $\mathrm{U}$ \\
\hline $75-27-4$ & Bromodichloromethane & 0.50 & $\mathrm{U}$ \\
\hline $10061-01-5$ & cis-1,3-Dichloropropene & 0.50 & $\mathrm{U}$ \\
\hline $108-10-1$ & 4-Methyl-2-pentanone & 5.0 & $\mathrm{U}$ \\
\hline $108-88-3$ & Toluene & 0.0087 & $\mathrm{~J}$ \\
\hline $10061-02-6$ & trans-1,3-Dichloropropene & 0.50 & $\mathrm{U}$ \\
\hline $79-00-5$ & $1,1,2-$ Trichloroethane & 0.50 & $\bar{U}$ \\
\hline $127-18-4$ & Tetrachloroethene & 0.50 & $\bar{U}$ \\
\hline $591-78-6$ & 2-Hexanone & 5.0 & $\bar{U}$ \\
\hline $124-48-1$ & Dibromochloromethane & 0.50 & $\mathrm{U}$ \\
\hline $106-93-4$ & 1,2-Dibromoethane & 0.50 & $\mathrm{U}$ \\
\hline $108-90-7$ & Chlorobenzene & 0.50 & $\bar{U}$ \\
\hline $100-41-4$ & Ethylbenzene & 0.50 & $\mathrm{U}$ \\
\hline $95-47-6$ & o-Xylene & 0.50 & $\bar{U}$ \\
\hline $179601-23-1$ & m,p-xylene & 0.50 & $\mathrm{U}$ \\
\hline $100-42-5$ & Styrene & 0.50 & $\bar{U}$ \\
\hline $75-25-2$ & Bromoform & 0.50 & $\mathrm{U}$ \\
\hline $98-82-8$ & Isopropylbenzene & 0.50 & $\mathrm{U}$ \\
\hline $79-34-5$ & $1,1,2,2$-Tetrachloroethane & 0.50 & $\mathrm{U}$ \\
\hline $541-73-1$ & 1,3-Dichlorobenzene & 0.030 & $\mathrm{~J}$ \\
\hline $106-46-7$ & 1,4-Dichlorobenzene & 0.50 & $\mathrm{U}$ \\
\hline $95-50-1$ & 1,2-Dichlorobenzene & 0.50 & $\mathrm{U}$ \\
\hline $96-12-8$ & 1,2-Dibromo-3-Chloropropane & 0.50 & $\mathrm{U}$ \\
\hline $120-82-1$ & $1,2,4$-Trichlorobenzene & 0.057 & $\mathrm{~J}$ \\
\hline $87-61-6$ & 1,2,3-Trichlorobenzene & 0.50 & $\mathrm{U}$ \\
\hline
\end{tabular}


IJ - FORM I VOA-TIC

VOLATILE ORGANICS ANALYSIS DATA SHEET TENTATIVELY IDENTIFIED COMPOUNDS
EPA SAMPLE NO.

VBLKDG

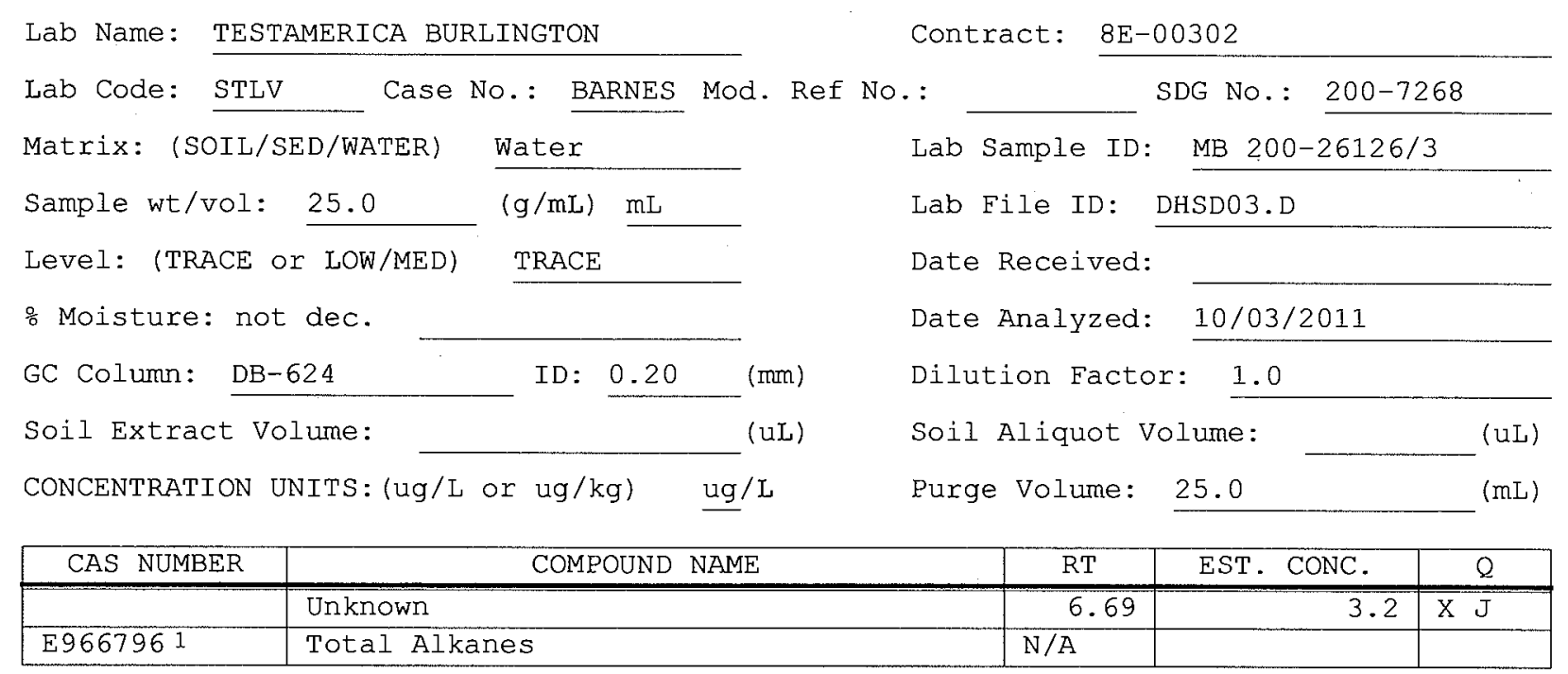

1 EPA-designated Registry Number. 
Lab Name: TESTAMERICA BURLINGTON

Lab Code: STLV

Case No.: BARNES Mod. Ref No.:
Contract: $\quad 8 \mathrm{E}-00302$

SDG No.: $200-7268$
Matrix: (SOIL/SED/WATER) Water

Sample wt/vol: 25.0

$(\mathrm{g} / \mathrm{mL}) \quad \mathrm{mL}$

Level: (TRACE/LOW/MED). TRACE

\% Moisture: not dec.

GC Column: DB-624 ID: $0.20 \quad(\mathrm{~mm})$

Soil Extract Volume: (uL) (mL)

Purge Volume: 25.0
Lab Sample ID: $200-7268-5$

Lab File ID: DHSD15.D

Date Received:

Date Analyzed: 10/03/2011

Dilution Factor: 1.0

Soil Aliquot volume:

(uL)

\begin{tabular}{|c|c|c|c|}
\hline CAS NO. & COMPOUND & $\begin{array}{l}\text { CONCENTRATION UNITS: } \\
(\mathrm{ug} / \mathrm{L} \text { or } u g / \mathrm{kg}) \mathrm{ug} / \mathrm{L}\end{array}$ & - Q \\
\hline $75-71-8$ & Dichlorodifluoromethane & 0.50 & $\mathrm{U}$ \\
\hline $74-87-3$ & Chloromethane & 0.50 & $\mathrm{U}$ \\
\hline $75-01-4$ & Vinyl chloride & 0.50 & $\mathrm{U}$ \\
\hline $74-83-9$ & Bromomethane & 0.50 & $\mathrm{U}$ \\
\hline $75-00-3$ & Chloroethane & 0.50 & $\mathrm{U}$ \\
\hline $75-69-4$ & Trichlorofluoromethane & 0.50 & U \\
\hline $75-35-4$ & 1,1-Dichloroethene & 0.50 & $\mathrm{U}$ \\
\hline $76-13-1$ & 1,1,2-Trichloro-1,2,2-trifluoroethane & 0.50 & $\mathrm{U}$ \\
\hline $67-64-1$ & Acetone & 2.4 & $\mathrm{JB}$ \\
\hline $75-15-0$ & Carbon disulfide & 0.50 & $\mathrm{U}$ \\
\hline $79-20-9$ & Methyl acetate & 0.50 & $\mathrm{U}$ \\
\hline $75-09-2$ & Methylene Chloride & 0.50 & $\mathrm{U}$ \\
\hline $156-60-5$ & trans-1,2-Dichloroethene & 0.50 & $\mathrm{U}$ \\
\hline $1634-04-4$ & Methyl tert-butyl ether & 0.50 & $\mathrm{U}$ \\
\hline $75-34-3$ & 1,1-Dichloroethane & 0.50 & $\mathrm{U}$ \\
\hline $156-59-2$ & cis-1,2-Dichloroethene & 0.50 & $\mathrm{U}$ \\
\hline $78-93-3$ & 2-Butanone & 5.0 & $\mathrm{U}$ \\
\hline $74-97-5$ & Bromochloromethane & 0.50 & $\mathrm{U}$ \\
\hline $67-66-3$ & Chloroform & 0.50 & U \\
\hline $71-55-6$ & $1,1,1$-Trichloroethane & 0.50 & $\mathrm{U}$ \\
\hline $110-82-7$ & Cyclohexane & 0.50 & $\mathrm{U}$ \\
\hline $56-23-5$ & Carbon tetrachloride & 0.50 & $\mathrm{U}$ \\
\hline $71-43-2$ & Benzene & 0.50 & $\mathrm{U}$ \\
\hline $107-06-2$ & 1,2-Dichloroethane & 0.50 & $\mathrm{U}$ \\
\hline
\end{tabular}

Report 1,4-Dioxane for Low-Medium VOA analysis only 
$1 B$ - EORM I VOA-2

VOLATILE ORGANICS ANALYSIS DATA SHEET
EPA SAMPLE NO.

VHBLKO1
Lab Name: TESTAMERICA BURLINGTON

Lab Code: STLV Case

Matrix: (SOIL/SED/WATER)

Sample wt/vol: 25.0 )

Water $(\mathrm{g} / \mathrm{mL}) \mathrm{mL}$

Level: (TRACE/LOW/MED) TRACE

\% Moisture: not dec.

GC Column: DB-624 ID $0.20 \quad(\mathrm{~mm})$

Soil Extract Volume: (uL)

Contract: $8 \mathrm{E}-00302$

AARNES Mod. Ref No.: SDG No.: 200-7268

Purge Volume: 25.0

(mL)

Lab Sample ID: 200-7268-5

Lab File ID: DHSD15.D

Date Received:

Date Analyzed: 10/03/2011

Dilution Factor: 1.0

Soil Aliquot volume:

(uL)

\begin{tabular}{|c|c|c|c|}
\hline CAS NO. & COMPOUND & $\begin{array}{l}\text { CONCENTRATION UNITS: } \\
(\mathrm{ug} / \mathrm{L} \text { or } \mathrm{ug} / \mathrm{kg}) \mathrm{ug} / \mathrm{I}\end{array}$ & $Q$ \\
\hline $79-01-6$ & Trichloroethene & 0.50 & $\bar{U}$ \\
\hline $108-87-2$ & Methylcyclohexane & 0.50 & $\mathrm{U}$ \\
\hline $78-87-5$ & 1,2-Dichloropropane & 0.50 & $\mathrm{U}$ \\
\hline $75-27-4$ & Bromodichloromethane & 0.50 & $\mathrm{U}$ \\
\hline $10061-01-5$ & cis-1,3-Dichloropropene & 0.50 & $\mathrm{U}$ \\
\hline $108-10-1$ & 4-Methyl-2-pentanone & 5.0 & $\mathrm{U}$ \\
\hline $108-88-3$ & Toluene & 0.50 & $\mathrm{U}$ \\
\hline $10061-02-6$ & trans-1,3-Dichloropropene & 0.50 & $\mathrm{U}$ \\
\hline $79-00-5$ & $1,1,2$-Trichloroethane & 0.50 & $\mathrm{U}$ \\
\hline $127-18-4$ & Tetrachloroethene & 0.50 & $\mathrm{U}$ \\
\hline $591-78-6$ & $2-$ Hexanone & 5.0 & $\mathrm{U}$ \\
\hline $124-48-1$ & Dibromochloromethane & 0.50 & $\mathrm{U}$ \\
\hline $106-93-4$ & 1,2-Dibromoethane & 0.50 & $\mathrm{U}$ \\
\hline $108-90-7$ & Chlorobenzene & 0.50 & $\mathrm{U}$ \\
\hline $100-41-4$ & Ethylbenzene & 0.50 & $\mathrm{U}$ \\
\hline $95-47-6$ & o-Xylene & 0.50 & $\mathrm{U}$ \\
\hline $179601-23-1$ & $\mathrm{~m}, \mathrm{p}$-xylene & 0.50 & $\mathrm{U}$ \\
\hline $100-42-5$ & Styrene & 0.50 & $\mathrm{U}$ \\
\hline $75-25-2$ & Bromoform & 0.50 & $\mathrm{U}$ \\
\hline $98-82-8$ & Isopropylbenzene & 0.50 & $\mathrm{U}$ \\
\hline $79-34-5$ & $1,1,2,2$-Tetrachloroethane & 0.50 & $\mathrm{U}$ \\
\hline $541-73-1$ & 1,3-Dichlorobenzene & 0.50 & $\mathrm{U}$ \\
\hline $106-46-7$ & 1,4-Dichlorobenzene & 0.50 & $\mathrm{U}$ \\
\hline $95-50-1$ & 1,2-Dichlorobenzene & 0.50 & $\mathrm{U}$ \\
\hline $96-12-8$ & 1,2-Dibromo-3-Chloropropane & 0.50 & $\mathrm{U}$ \\
\hline $120-82-1$ & 1,2,4-Trichlorobenzene & 0.50 & $\mathrm{U}$ \\
\hline $87-61-6$ & $1,2,3-$ Trichlorobenzene & 0.50 & $\mathrm{U}$ \\
\hline
\end{tabular}


IJ - FORM I VOA-TIC

VOLATILE ORGANICS ANALYSIS DATA SHEET TENTATIVELY IDENTIFIED COMPOUNDS
EPA SAMPLE NO.

VHBLKOI

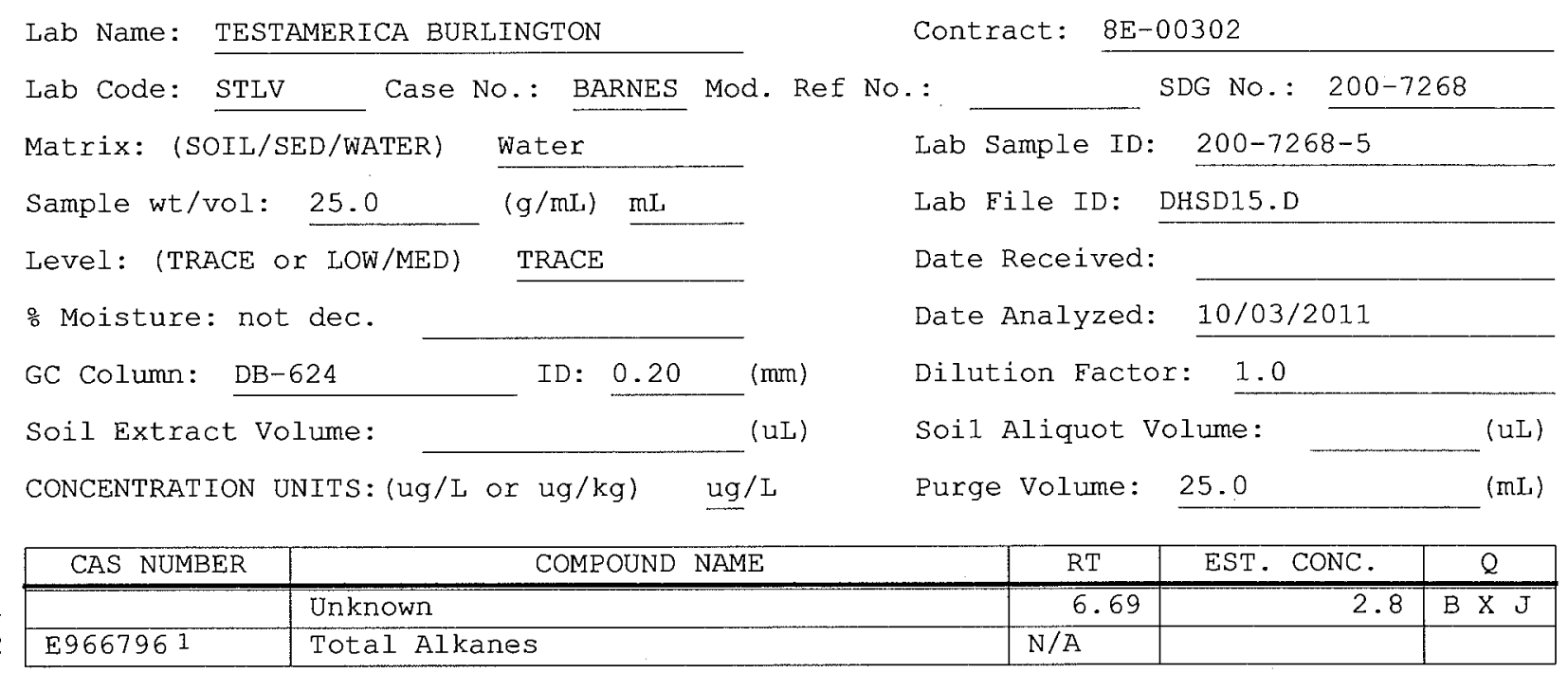

1 EPA-designated Registry Number. 
Argonne

\section{Environmental Science Division}

Argonne National Laboratory

9700 South Cass Avenue, Bldg. 203

Argonne, IL 60439-4843

www.anl.gov 